Universidad Politécnica de Cartagena

Departamento de Matemática Aplicada y Estadística

\title{
Mejora de la Maniobrabilidad de Submarinos Tripulados Mediante Control Óptimo de Tanques de Lastre
}

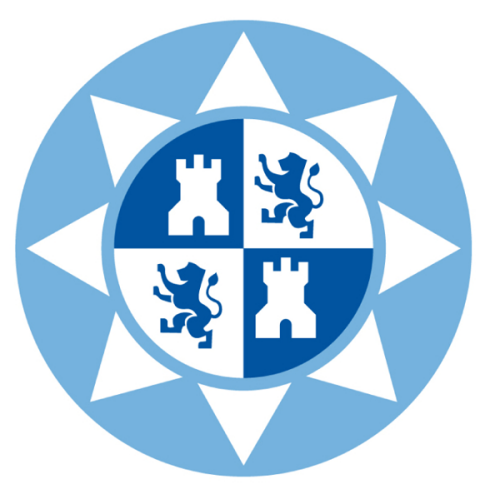

Roberto Javier Font Ruiz 



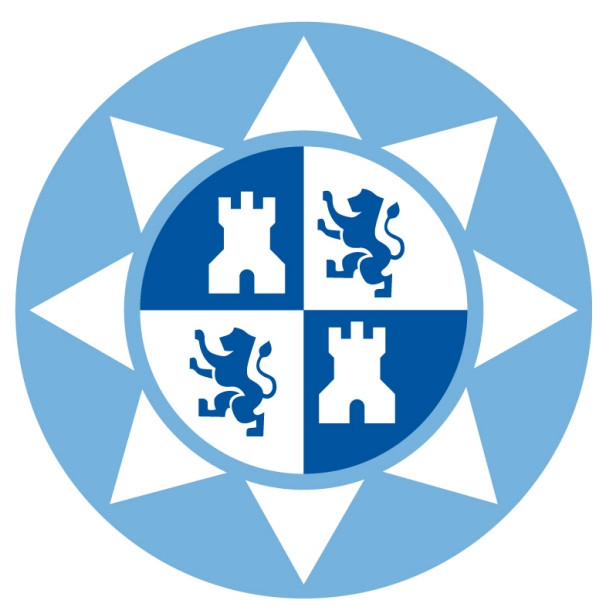

Universidad Politécnica de Cartagena

Departamento de Matemática Aplicada y Estadística

\section{Mejora de la Maniobrabilidad de Submarinos Tripulados Mediante Control Óptimo de Tanques de Lastre}

Roberto Javier Font Ruiz

Directores

Francisco Periago Esparza

Pablo Pedregal Tercero 



\section{CONFORMIDAD DE SOLICITUD DEAUTORIZACIÓN DE DEPÓSITO DE} TESIS DOCTORAL POR EL/LA DIRECTOR/A DE LA TESIS

D./D ${ }^{\mathrm{a}}$ FRANCISCO PERIAGO ESPARZA Director/a de la Tesis doctoral MEJORA DE LA MANIOBRABILIDAD DE SUBMARINOS TRIPULADOS MEDIANTE CONTROL ÓPTIMO DE TANQUES DE LASTRE

\section{INFORMA:}

Que la referida Tesis Doctoral, ha sido realizada por D/D ${ }^{a}$. ROBERTO JAVIER FONT RUIZ, dando mi conformidad para que sea presentada ante la Comisión de Doctorado, para ser autorizado su depósito.

La rama de conocimiento por la que esta tesis ha sido desarrollada es:

0 Ciencias

4 Ciencias Sociales y Jurídicas

\&' Ingeniería y Arquitectura

En Cartagena, a 3 de diciembre de 2012

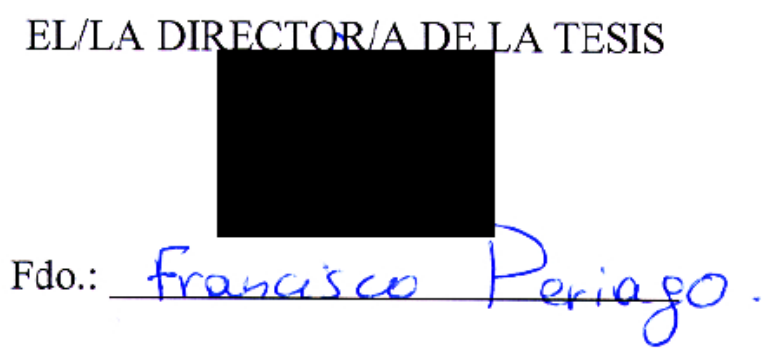





\section{Universidad}

Politécnica

de Cartagena

DT-16

CONFORMIDAD DE SOLICITUD DEAUTORIZACIÓN DE DEPÓSITO DE TESIS DOCTORAL POR EL/LA DIRECTOR/A DE LA TESIS

D./Da . PABLO PEDREGAL TERCERO Director/a de la Tesis doctoral

MEJORA DE LA MANIOBRABILIDAD DE SUBMARINOS TRIPULADOS

MEDIANTE CONTROL ÓPTIMO DE TANQUES DE LASTRE

\section{INFORMA:}

Que la referida Tesis Doctoral, ha sido realizada por D/Da. ROBERTO JAVIER FONT RUIZ, dando mi conformidad para que sea presentada ante la Comisión de Doctorado, para ser autorizado su depósito.

La rama de conocimiento por la que esta tesis ha sido desarrollada es:

国 Ciencias

Ciencias Sociales y Jurídicas

Ingeniería y Arquitectura

En Ciudad Real, a 3 de diciembe de 2012

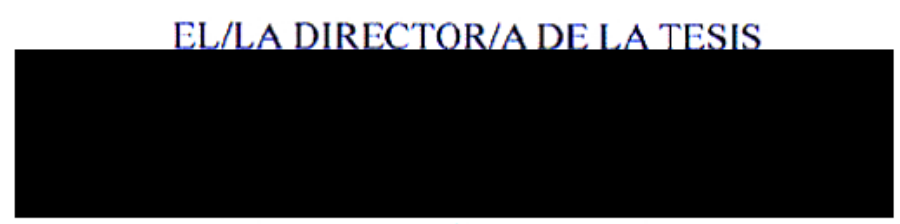

Fdo.: Pablo Pedregal 



\section{Universidad}

Politécnica

de Cartagena

DT-17

\section{CONFORMIDAD DE DEPÓSITO DE TESIS DOCTORAL POR LA COMISIÓN ACADÉMICA DEL PROGRAMA}

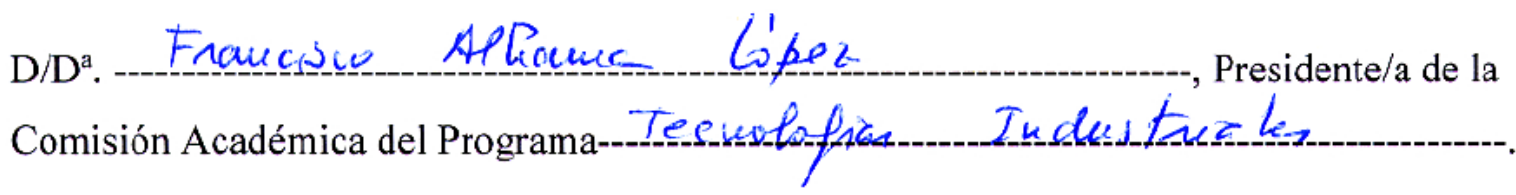

\section{INFORMA:}

Que la Tesis Doctoral titulada, "MEJORA DE LA MANIOBRABILIDAD DE SUBMARINOS TRIPULADOS MEDIANTE CONTROL ÓPTIMO DE TANQUES DE LASTRE", ha sido realizada por D/Da. ROBERTO JAVIER FONT RUIZ,

bajo la dirección y supervisión del Dr FRANCISCO PERIAGO ESPARZA Y PABLO PEDREGAL TERCERO.

En reunión de la Comisión Académica de fecha $\int_{f-2}$ 2012, visto que la mencionada tesis doctoral tiene acreditados los indicios de calidad, requeridos para el depósito de tesis doctorales, regulados en el artículo 32 del Reglamento de Estudios Oficiales de Máster y Doctorado de la UPCT, y la autorización del Director de la misma, se acordó dar la conformidad para que a dicha tesis le sea autorizado, por la Comisión de Doctorado, su depósito.

La Rama de conocimiento por la que esta tesis ha sido desarrollada es:

$\square$ Ciencias

$\square$ Ciencias Sociales y Jurídicas

$\square$ Ingeniería y Arquitectura

En Cartagena, a -19-de Xrie- de-2012

EL PRESIDENTE DE LA COMISIÓN ACADÉMICA DEL PROGRAMA

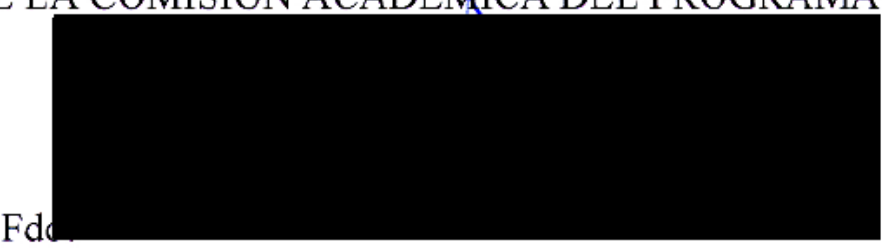



Deseo expresar mi más profundo agradecimiento a mis directores, por su dedicación, su entusiasmo, su guía, y por enseñarme todo cuanto sé; a Javier García Peláez, por guiarnos en aguas hostiles y responder todas nuestras preguntas, y a los miembros del grupo OMEVA, de la Universidad de Castilla-La Mancha, y del grupo de Ecuaciones Diferenciales y Análisis Numérico, de la Universidad Politécnica de Cartagena, por el privilegio de participar en tantas estimulantes conversaciones.

Gracias también a mi familia y amigos, y sobre todo a María Amelia, matemática de cabecera, por estar ahí cada día.

Este trabajo ha sido financiado mediante una Beca de Iniciación en la Investigación de la Universidad Politécnica de Cartagena y los proyectos 2989/10MAE entre Navantia S. A. y la Universidad Politécnica de Cartagena y 08720/PI/08 de la Fundación Séneca, Agencia de Ciencia y Tecnología de la Región de Murcia. 



\section{Abstract}

Manned submarines are equipped with a number of ballast tanks distributed along the hull. When these tanks are filled with water, they contribute with the submarine mass allowing it to submerge. In case of emergency they can act as a safety mechanism to drive the vehicle to surface: air is blown into the ballast tanks from very high pressure bottles expelling the water out of the tanks. This way the submarine loses weight, its buoyancy is higher, and it can emerge quicker. To fill the tanks with water, a valve located on the top of the tanks is opened, air escapes outside, and water flows into the tanks. These processes are known, respectively, as blowing and venting of ballast tanks.

Our aim is to study these processes providing all the necessary mathematical tools for their analysis, simulation and control. As a second step, we explore the potential use of these processes as a complementary control mechanism that could improve the manoeuvrability of manned submarines in certain situations. Particularly, we address the improvement of safety and stability in emergency rising manoeuvres and the control of hovering manoeuvres. Accurate hovering, the action of statically keep a desired depth, is essential, for example, for the launch and recovery of Autonomous Underwater Vehicles (AUVs), a subject that has recently raised an extraordinary interest.

We first propose a mathematical model for the blowing and venting processes which is coupled with the usual 6 degree of freedom kinematic and dynamic equations of motion. A rigorous mathematical analysis of the resulting state law is carried out. The control problem is formulated as a Bolza-type optimal control problem. Existence of solution for this problem is proved and the numerical resolution using a gradient descent method is addressed. We then propose a feedback control scheme for the tracking of the optimal trajectories obtained as a solution of this optimal control problem. For the hovering control, we propose a control law consisting of a sliding mode controller acting on a previously input-output exactly linearized system.

The numerical simulations carried out show that, indeed, an appropriate control of these processes can help in a significant way to improve stability and safety during emergency manoeuvres as well as be an invaluable tool for the hovering control. 



\section{Resumen}

Los submarinos tripulados están equipados con un cierto número de tanques de lastre distribuidos a lo largo de su casco que, cuando están llenos de agua, proporcionan al submarino el peso necesario para sumergirse. En caso de emergencia, los tanques de lastre pueden actuar como un mecanismo de seguridad, permitiendo llevar el vehículo a la superficie con rapidez. Para ello, se inyecta en los tanques aire a alta presión que fuerza la salida del agua al exterior. De este modo el submarino pierde peso, su flotabilidad aumenta, y puede emerger más rápidamente. Para el llenado de los tanques, se abre una válvula localizada en su parte superior, de forma que el aire escapa al exterior permitiendo la entrada de agua. Estos procesos se conocen, respectivamente, como soplado y ventilación de los tanques de lastre.

Nuestro propósito es estudiar estos procesos proporcionando, en primer lugar, las herramientas matemáticas necesarias para su análisis, simulación y control. Analizamos, en segundo lugar, el posible uso de estos procesos como un mecanismo de control adicional que permita mejorar la maniobrabilidad del vehículo en determinadas situaciones. Estudiamos, en concreto, el aumento de la estabilidad durante maniobras de ascenso de emergencia y la posibilidad de realizar hovering, es decir, de mantener la cota en ausencia de velocidad de avance. Esta última capacidad resulta esencial, por ejemplo, para el lanzamiento y recogida de vehículos autónomos (AUVs), un asunto que ha despertado un enorme grado de interés en los últimos tiempos.

Proponemos en primer lugar un modelo matemático para los procesos de soplado y ventilación acoplado con las ecuaciones cinemáticas y dinámicas del movimiento del vehículo. Realizamos un riguroso análisis matemático de la ley de estado resultante. Formulamos el problema de control como un problema de control óptimo de Bolza para el que probamos la existencia de solución y que es resuelto mediante un método de descenso tipo gradiente. A continuación proponemos un algoritmo de control en lazo cerrado que permite seguir en presencia de incertidumbres las trayectorias óptimas obtenidas como solución de este problema de Bolza. Para el estudio del hovering, se propone una ley de control basada en el control en modo deslizante.

Las simulaciones numéricas llevadas a cabo muestran que, en efecto, el uso de los procesos de soplado y ventilación como un mecanismo de control puede ser una herramienta de gran valía tanto en el control del hovering como para la mejora de la seguridad durante maniobras de emergencia. 



\section{Índice general}

$\begin{array}{ll}\text { Introducción } & \text { XXI }\end{array}$

I Modelos Matemáticos $\quad 1$

1. Ecuaciones del movimiento de un vehículo submarino 3

1.1. El submarino $\mathrm{P}-650 \ldots \ldots \ldots \ldots \ldots \ldots$

1.2. Grados de libertad y sistemas de referencia . . . . . . . . . . . . 5

1.3. Transformación de coordenadas . . . . . . . . . . . . . . . . . 6

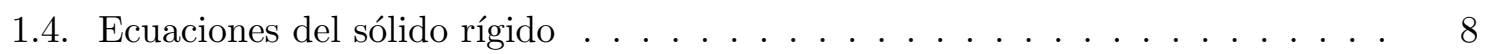

1.5. Fuerzas y momentos hidrodinámicos . . . . . . . . . . . . . . 9

1.6. Ecuaciones del movimiento . . . . . . . . . . . . . . . . . 11

1.6.1. Ecuaciones cinemáticas . . . . . . . . . . . . . . . . . . 11

1.6.2. Ecuaciones dinámicas . . . . . . . . . . . . . . . 11

$\begin{array}{ll}\text { 2. Perturbaciones externas } & 17\end{array}$

2.1. Fuerzas inducidas por el oleaje . . . . . . . . . . . . . . . 17

2.1.1. Mar irregular . . . . . . . . . . . . . . . . . . . 18

2.1.2. Esfuerzos sobre el submarino . . . . . . . . . . . . . . . . . 24

2.2. Flotabilidad variable . . . . . . . . . . . . . . . . . . . 31

2.2.1. Efecto de la compresibilidad de los materiales . . . . . . . . . . 31

2.2.2. Efecto del gradiente de densidad . . . . . . . . . . . . . . 35

$\begin{array}{lll}\text { 3. Soplado y ventilación de tanques de lastre } & 37\end{array}$

3.1. Tanques de lastre . . . . . . . . . . . . . . . . . . . 38 
3.2. Flujo de aire desde la botella . . . . . . . . . . . . . . . . . . . . 40

3.3. Flujo de aire a través del conducto de ventilación . . . . . . . . . . . . . . 43

3.4. Flujo de agua a través del orificio . . . . . . . . . . . . . . . . . . . . . 44

3.5. Evolución de la presión en el tanque . . . . . . . . . . . . . . . . . . . 45

3.6. Control del sistema de soplado-ventilación . . . . . . . . . . . . . . . . . . 46

3.7. Masa variable . . . . . . . . . . . . . . . . . . . . . . . 47

4. Forma final del modelo completo. Análisis matemático 53

4.1. Modelo completo en forma compacta . . . . . . . . . . . . . . . . . . 53

4.2. Análisis matemático . . . . . . . . . . . . . . . . . . . . . . 54

4.2.1. El vector de estado es acotado . . . . . . . . . . . . . . 55

4.2.2. La ley de estado está bien planteada . . . . . . . . . . . . 56

4.3. Limitaciones y posibles mejoras . . . . . . . . . . . . . . . . . . 63

$\begin{array}{ll}\text { II } & \text { Algoritmos de Control }\end{array}$

5. Control óptimo de tanques de lastre $\quad 69$

5.1. Formulación del problema de control óptimo . . . . . . . . . . . . . 70

5.2. Existencia de solución del problema de control óptimo . . . . . . . . . . . . 70

5.2.1. Soplado y ventilación de tanques y superficies de control actuando de forma conjunta . . . . . . . . . . . . . . . . . 71

5.3. Resolución numérica . . . . . . . . . . . . . . . . . . . . . 72

6. Algoritmo feedback para el seguimiento de trayectorias óptimas $\quad 75$

6.1. Formulación del problema . . . . . . . . . . . . . . . . . . . . . 76

6.2. Esquema numérico . . . . . . . . . . . . . . . . . . . . . 77

6.3. Un ejemplo académico . . . . . . . . . . . . . . . . . . 78

6.4. Posible extensión del método . . . . . . . . . . . . . . . . . . 84

7. Control en modo deslizante de sistemas no lineales $\quad 89$

7.1. Linealización feedback . . . . . . . . . . . . . . . . . . . . . . . . . . 90

7.1.1. Linealización input-output de sistemas de una entrada y una salida $(\mathrm{SISO}) \ldots \ldots \ldots \ldots \ldots \ldots$ 
7.1.2. Sistemas con múltiples entradas y múltiples salidas (MIMO) _ . . . . 92

7.2. Control en modo deslizante . . . . . . . . . . . . . . . . . . . 93

7.3. Control de los procesos de soplado y ventilación . . . . . . . . . . . . . . . 96

\section{Resultados}

8. Maniobras de ascenso de emergencia

8.1. Una maniobra de ascenso de emergencia . . . . . . . . . . . . . . . . . . 102

8.1.1. Análisis en lazo abierto . . . . . . . . . . . . . . . . . . . . 102

8.1.2. Control feedback . . . . . . . . . . . . . . . . . . . . . . . 107

8.2. Bloqueo del timón horizontal de popa durante un cambio de rumbo . . . . . . 113

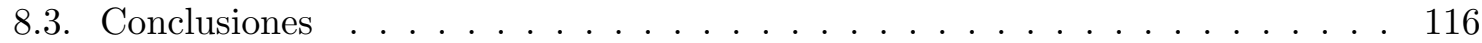

9. Maniobras a baja o nula velocidad. Hovering 117

9.1. Sistema de hovering . . . . . . . . . . . . . . . . . . . . . . 118

9.2. Modelo matemático para el control mediante bombas hidráulicas . . . . . . . 120

9.3. Simulaciones numéricas _. . . . . . . . . . . . . . . . . . 120

9.3.1. Escenario 9.1: Cambios bruscos de densidad. Control óptimo . . . . . 121

9.3.2. Escenario 9.2: Esfuerzos producidos por el oleaje. Control en modo deslizante . . . . . . . . . . . . . . . . . 125

9.4. Conclusiones . . . . . . . . . . . . . . . . . . . . 125

$\begin{array}{lr}\text { Conclusiones } & 129\end{array}$

Anexo. Ajuste experimental de los esfuerzos producidos por el oleaje $\quad 131$

$\begin{array}{ll}\text { Bibliografía } & 147\end{array}$ 



\section{Índice de tablas}

1.1. Características principales del submarino $\mathrm{P}-650 \ldots \ldots$. . . . . . . . . 4

1.2. Notación de la SNAME para vehículos submarinos . . . . . . . . . 6

1.3. Parámetros geométricos del submarino $\mathrm{P}-650 \ldots \ldots$. . . . . . . . 13

1.4. Coeficientes del modelo de la hélice . . . . . . . . . . . . . . . . . . . . . 14

1.5. Coeficientes hidrodinámicos adimensionales . . . . . . . . . . . . . 15

2.1. Escala Douglas para la descripción de los diferentes estados de mar . . . . . 20

2.2. Valores de $H_{1 / 3}$ y $T_{z}$ para diferentes estados de mar en el Mediterráneo . . . 20

2.3. Valor y significado de los parámetros empleados por el modelo de flotabilidad

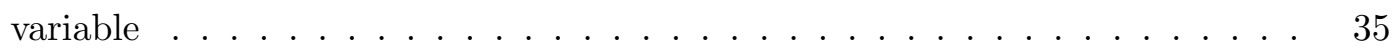

3.1. Variables y símbolos . . . . . . . . . . . . . . . . . 39

3.2. Características geométricas de los tanques de lastre . . . . . . . . . . . . 48

6.1. Valores del coste óptimo $J_{h}$ para diferentes valores de $h \ldots \ldots$. . . . . 80

8.1. Valor del coste obtenido con controles óptimos y estándar . . . . . . . . . . . 104

8.2. Valor de la función coste para diferentes periodos de muestreo . . . . . . . . . 109 



\section{Índice de figuras}

1.1. Modelo a escala del submarino $\mathrm{P}-650 \ldots \ldots$. . . . . . . . . . . . 4

1.2. Localización de las superficies de control . . . . . . . . . . . . . 5

1.3. Variables y sistemas de referencia . . . . . . . . . . . . . 7

1.4. Peso y flotabilidad . . . . . . . . . . . . . . . . . 10

2.1. Parámetros principales de una onda regular . . . . . . . . . . . . . . . 18

2.2. Distribución de la energía a lo largo del espectro de frecuencias para diferentes estados de $\operatorname{mar} \ldots \ldots \ldots \ldots \ldots . \ldots \ldots \ldots$

2.3. Ángulo relativo entre vehículo y oleaje . . . . . . . . . . . . . . . . 22

2.4. Simulación de un estado de mar 3 en un área de 200 x 200 m empleando el modelo long crested (izquierda) y short crested (derecha) . . . . . . . . . . .

2.5. Evolución a lo largo del tiempo de la elevación superficial en el origen empleando los modelos long crested (arriba) y short crested (abajo) . . . . . . . 23

2.6. Módulo y fase de la fuerza vertical para ángulo de encuentro de $135^{\circ} \ldots \ldots$

2.7. Amplitud de la fuerza vertical (arriba) y el momento de giro (abajo) en función de la longitud de onda para ángulo de encuentro de $180^{\circ} \ldots \ldots 26$

2.8. Elevación superficial, fuerza vertical y momento de giro para estado de mar 5 con ángulo de encuentro $\chi=180^{\circ}$ y velocidad $U=6 \mathrm{kn} \ldots \ldots . \ldots 28$

2.9. Elevación superficial, fuerza vertical y momento de giro para estado de mar 5 con ángulo de encuentro $\chi=45^{\circ}$ y velocidad $U=6 \mathrm{kn} \ldots \ldots \ldots$. . . . .

2.10. Pérdida de flotabilidad debida a la compresión de los aislantes acústicos en función de la cota . . . . . . . . . . . . . . . . . . . 34

3.1. Distribución de los tanques de lastre en el submarino $\mathrm{P}-650 \ldots$. . . . . . . 38

3.2. Vista esquemática de los procesos de soplado y ventilación . . . . . . . . . . . 40 
3.3. Resultado del ajuste por mínimos cuadrados de $\bar{\mu}(\Pi)$ teniendo en cuenta las pérdidas de presión . . . . . . . . . . . . . . . . . . 44 44

3.4. Localización de la pérdida de masa . . . . . . . . . . . . . . . . . . 49

6.1. Representación del conjunto en el que $\phi$ es finito y la solución óptima de (VP $\left.{ }^{1}\right) 79$

6.2. Control óptimo feedback $u(t), 0 \leq t \leq 1$ y estado asociado $x(t), 0 \leq t \leq 1$ para $h=0,1$ y $h=0,001 \ldots \ldots \ldots \ldots$. . . . . . . . . . . 80

6.3. Variable de control $u(t)$ y estado $x(t), 0 \leq t \leq 1$ no perturbada y afectada por ruido blanco aditivo con $\sigma=0,1, \sigma=0,001$ y $\sigma=0,0001 \ldots \ldots \ldots$

6.4. Resultados para el error inducido en la variable de control por una perturbación aleatoria de la variable de estado . . . . . . . . . . . . . . . . . . 83

6.5. Elección de un horizonte $T>h$ para la optimización . . . . . . . . . . . 86

6.6. Comparación de los resultados obtenidos para un cambio de rumbo a $5 \mathrm{kn}$ empleando $h=1$ y $T=1,3,5,10,20 \ldots \ldots \ldots \ldots$

7.1. Comportamiento de un sistema bajo SMC. Oscilaciones conocidas como chat-

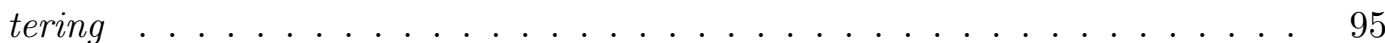

7.2. Representación de la función $\operatorname{sat}(\cdot)$ para $\Phi=0,2 \ldots \ldots \ldots$. . . . . 96

8.1. Evolución de la cota y los ángulos de pitch y roll. Escenarios 8.1-8.3 . . . . . 105

8.2. Apertura de las válvulas de soplado. Escenarios 8.1-8.3 . . . . . . . . . . . . 106

8.3. Apertura de las válvulas de ventilación. Escenarios 8.1-8.3 . . . . . . . . . . 106

8.4. Evolución del coste para el Escenario 8.3 . . . . . . . . . . . . . . . . . 107

8.5. Escenario 8.4. Evolución de la cota y los ángulos de pitch y roll . . . . . . . . 110

8.6. Escenario 8.5. Evolución de la cota y los ángulos de pitch y roll . . . . . . . . 111

8.7. Apertura de las válvulas de soplado para la trayectoria óptima y los controles feedback. Escenarios 8.4 y 8.5 . . . . . . . . . . . . . . . . . 112

8.8. Apertura de las válvulas de ventilación para la trayectoria óptima y los controles feedback. Escenarios 8.4 y 8.5 . . . . . . . . . . . . . . . . 112

8.9. Evolución de la cota y el ángulo de roll . . . . . . . . . . . . . . . . . . . . 114

8.10. Deflexión del timón vertical y el plano horizontal de proa . . . . . . . . . . 115

8.11. Apertura de las válvulas de soplado . . . . . . . . . . . . . . . . . . 116

9.1. Gradiente de temperatura para el Escenario 9.1 . . . . . . . . . . . . . . . 122 
9.2. Comparación de la evolución de la cota para el Escenario 9.1 empleando control mediante bombeo y soplado/ventilación . . . . . . . . . . . . . . . .

9.3. Volumen inundado en los tanques, caudal bombeado y aperturas de las válvulas de soplado y ventilación . . . . . . . . . . . . . . . . . . . . . . . . . . 124

9.4. Comparación de la evolución de la cota para el Escenario 9.2 empleando control mediante bombeo y soplado/ventilación . . . . . . . . . . . . . . 126

9.5. Caudal bombeado y apertura de las válvulas de soplado y ventilación . . . . 127 



\section{Introducción}

Los submarinos convencionales como el nuevo S-80, que en la actualidad construye la empresa Navantia S. A. para la Armada Española, son habitualmente gobernados mediante uno o varios propulsores, que proporcionan la velocidad de avance, y una serie de superficies de control o timones que controlan su movimiento en los planos horizontal y vertical. De forma complementaria, los submarinos tripulados están equipados con un cierto número de tanques de lastre distribuidos a lo largo de su casco. Cuando estos tanques están llenos de agua, proporcionan al submarino el peso necesario para sumergirse. En caso de emergencia, los tanques de lastre pueden actuar como un mecanismo de seguridad, permitiendo llevar el vehículo a la superficie con rapidez. Para ello, se inyecta en los tanques aire a alta presión que fuerza la salida del agua al exterior. De este modo el submarino pierde peso, su flotabilidad aumenta, y puede emerger más rápidamente. Para el llenado de los tanques, se abre una válvula localizada en su parte superior, de forma que el aire escapa al exterior permitiendo la entrada de agua. Estos procesos se conocen, respectivamente, como soplado y ventilación de los tanques de lastre.

El soplado de emergencia es considerado potencialmente peligroso, por lo que muy rara vez se realizan pruebas de mar de este tipo de maniobras. El ensayo con modelos a escala, por otra parte, puede alcanzar costes prohibitivos para muchas armadas y, en cualquier caso, justificables sólo durante las etapas finales del desarrollo de un nuevo vehículo. En este contexto, la simulación numérica representa una alternativa considerablemente más accesible y una herramienta de enorme utilidad, particularmente durante las etapas iniciales de diseño o para la experimentación de nuevas ideas y conceptos. Ésta es la principal motivación del presente trabajo.

Nuestro primer objetivo es obtener modelos matemáticos para los procesos de soplado y ventilación y su influencia sobre la dinámica del vehículo. La capacidad de simular maniobras que involucren el soplado y ventilación de los tanques puede contribuir a mejorar la comprensión de este tipo de maniobras de emergencia y sus dificultades asociadas y permite a los ingenieros, durante la fase de diseño de un nuevo vehículo, ajustar los valores de los 
diferentes parámetros geométricos implicados en los sistemas de soplado y ventilación, como el tamaño y posición de los tanques o los diámetros de las válvulas.

Nuestro segundo objetivo es analizar la posibilidad de emplear los procesos de soplado y ventilación como un mecanismo de control adicional que permita mejorar la maniobrabilidad del vehículo. El control de la flotabilidad puede ser especialmente relevante en situaciones en las que, debido a la baja velocidad, los medios tradicionales de control no son efectivos. En este sentido, resulta particularmente interesante dotar al submarino de la capacidad de hovering, es decir, la habilidad de mantener estáticamente su cota en ausencia de velocidad de avance. Esta capacidad resulta esencial, por ejemplo, para el lanzamiento y recogida desde el submarino de vehículos no tripulados, una posibilidad que ha despertado en los últimos tiempos un creciente grado de interés por parte de las principales armadas.

Esta memoria está organizada en tres partes, dedicadas de forma respectiva a los modelos matemáticos, los algoritmos de control empleados y los resultados numéricos. En el Capítulo 1 se recogen las ecuaciones cinemáticas y dinámicas para el movimiento de un vehículo submarino. El Capítulo 2 expone los modelos matemáticos que serán empleados para la simulación de perturbaciones en el entorno del vehículo. En ambos casos, se han adaptado a las características particulares del vehículo en consideración modelos bien conocidos en la literatura naval.

En el Capítulo 3 proponemos un modelo matemático para los procesos de soplado y ventilación de tanques de lastre así como su influencia sobre el comportamiento del vehículo a través de las variaciones causadas en la masa y otras propiedades presentes en las ecuaciones dinámicas. El modelo para el soplado está basado en el esbozado en [Bys04] y ha probado, en opinión de los autores, tener la capacidad de capturar fenómenos que eran pasados por alto por los modelos anteriormente disponibles (véase [FGO10]). Hasta donde sabemos, el proceso de ventilación no ha sido tratado con anterioridad y, lo que es más importante, nunca antes se ha considerado el uso conjunto de ambos procesos como mecanismo de control. Los resultados originales de este capítulo pueden encontrarse en [FGPMP12].

En el Capítulo 4 se integran los modelos anteriores en una única forma compacta y se realiza un análisis matemático del sistema resultante. Este análisis, que tendrá gran importancia a la hora de probar la existencia de solución para el problema de control óptimo (Capítulo 5) está así mismo incluido en [FGPMP12].

El Capítulo 5 trata el control del vehículo mediante soplado y ventilación empleando Control Óptimo. Para ello, el problema de control es formulado como un problema de Bolza que es resuelto empleando un método de descenso tipo gradiente. El capítulo incluye un resultado de existencia de solución para este problema (Teorema 5.1). Los resultados de este capítulo están recogidos en [FGPMP12].

En el Capítulo 6 se propone un algoritmo en lazo cerrado para el seguimiento de las trayectorias óptimas generadas mediante la metodología descrita en el Capítulo 5. Este algoritmo, 
basado en la reformulación variacional de problemas de control óptimo, ha sido publicado en $[\mathrm{FPP}]$.

El Capítulo 7 hace una revisión de la linealización feedback y el control en modo deslizante; conocidas técnicas de control no lineal que serán empleadas en capítulos posteriores para el control del hovering mediante soplado/ventilación.

En el Capítulo 8 mostramos los resultados de la simulación numérica de maniobras de ascenso de emergencia empleando los algoritmos descritos en los capítulos 5 y 6 . Los resultados muestran que con un adecuado control el soplado diferencial de los tanques puede contribuir de manera sustantiva a mejorar la estabilidad y seguridad de este tipo de maniobras. Parte de los resultados de simulación incluidos en este capítulo han sido publicados en [FGPMP12, FPP].

El Capítulo 9 aborda el posible uso del soplado y ventilación de tanques para el control del hovering. Empleando los algoritmos de control óptimo y en modo deslizante descritos en los capítulos 5 y 7 respectivamente, se contrastan los resultados obtenidos mediante el sistema basado en soplado ventilación de tanques y un sistema tradicional basado en bombas hidráulicas. Los resultados muestran que el primero de estos sistemas es capaz de proporcionar resultados notablemente más precisos. Estos resultados se encuentran recogidos en [FGP12].

Cierra esta memoria un capítulo de conclusiones. 



\section{Parte I}

\section{Modelos Matemáticos}





\section{Ecuaciones del movimiento de un vehículo submarino}

Si bien las primeras simulaciones numéricas del movimiento de vehículos se remontan a la Segunda Guerra Mundial, cuando son empleadas para predecir el movimiento de dirigibles, no es hasta 1967 cuando aparece el primer conjunto de ecuaciones generalmente aceptado para la simulación de maniobras de vehículos submarinos [GH67]. Estas Standard Equations of Motion for Submarine Simulation desarrolladas por el David Taylor Naval Ship Research and Development Center fueron posteriormente revisadas por Feldman en 1979 [Fel79]. Ésta es la forma que ha permanecido como un estándar hasta la actualidad.

A lo largo de este capítulo revisamos el modelo matemático para el movimiento de un vehículo submarino proporcionando la forma final de las ecuaciones adaptadas a las características concretas del vehículo considerado a lo largo de este trabajo; el submarino P-650 de Navantia. Para una exposición más detallada del proceso de obtención de las ecuaciones del movimiento, se remite al lector a [Fos94, Fel79, Ova11] así como a las referencias allí proporcionadas.

Para la obtención de la forma habitual de las ecuaciones del movimiento suelen adoptarse las siguientes hipótesis de partida:

- El submarino se mueve como un sólido rígido e indeformable.

- Tanto la masa como la flotabilidad del vehículo permanecen constantes.

- El efecto de la rotación de la tierra puede considerarse despreciable. 
- El submarino se encuentra en inmersión, lejos de cualquier superficie libre o vehículo.

- No existen fuerzas externas actuando sobre el vehículo.

En capítulos posteriores serán revisadas algunas de estas hipótesis.

\subsection{El submarino $P-650$}

A lo largo de esta memoria emplearemos como referencia el submarino P-650 de Navantia, un diseño previo al S-80 actualmente en construcción. Si bien el submarino P-650 no llegó finalmente a construirse, sí se avanzó lo suficiente en su desarrollo como para efectuar un diseño detallado y para que se construyera un modelo a escala (Figura 1.1) sobre el que se realizaron numerosos tests y ensayos. Serán los datos procedentes de estos ensayos así como las características geométricas e hidrodinámicas de este vehículo las que emplearemos para la realización de simulaciones numéricas y el diseño de algoritmos de control.

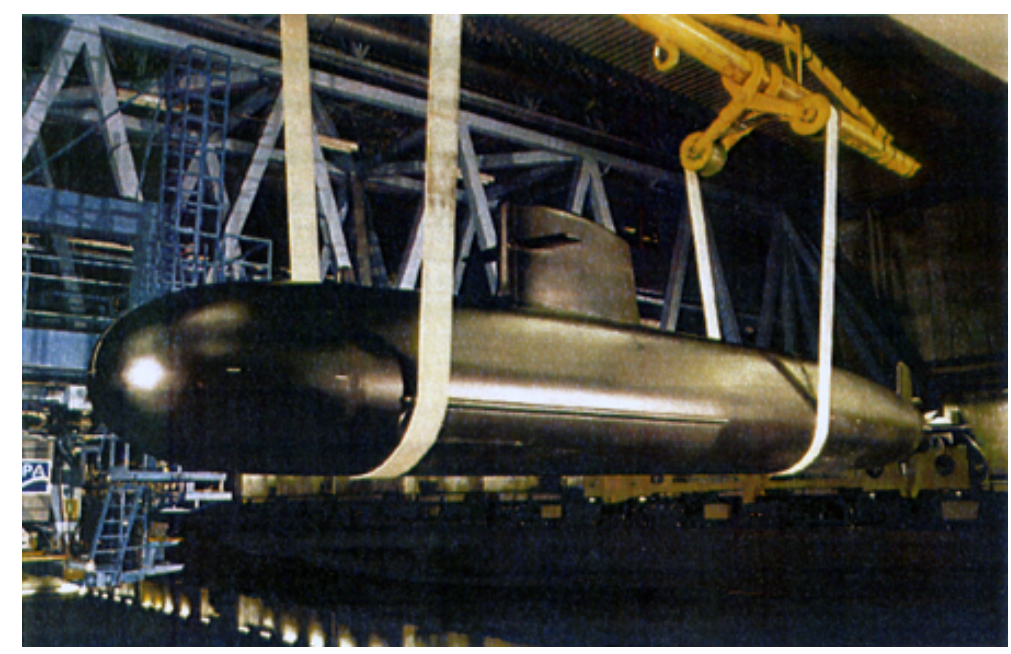

Figura 1.1: Modelo a escala del submarino P-650. Fotografía tomada de [Bys01a].

\begin{tabular}{|l|r|}
\hline Eslora total & $67 \mathrm{~m}$ \\
\hline Altura & $7.65 \mathrm{~m}$ \\
\hline Diámetro del casco & $6.6 \mathrm{~m}$ \\
\hline Desplazamiento en inmersión & 2352 ton \\
\hline Velocidad máxima & $20 \mathrm{kn}$ \\
\hline
\end{tabular}

Tabla 1.1: Características principales del submarino P-650.

El P-650 es un submarino de propulsión diésel-eléctrica de $67 \mathrm{~m}$ de longitud y un desplazamiento en inmersión de 2352 toneladas. La Tabla 1.1 resume algunas de sus principales 
características. El maniobrado de este vehículo se realiza mediante una única hélice situada en la popa, que proporciona la velocidad de avance, y tres superficies de control o timones cuya localización puede verse en la Figura 1.2. Un timón vertical situado en la popa, denominado rudder, que controla el movimiento en el plano horizontal de forma similar a los barcos de superficie y dos timones horizontales, o de buceo, que controlan el movimiento vertical. El primero de ellos (stern plane) está situado en la popa mientras que el segundo (bow plane) suele estar situado en la proa o, como es nuestro caso, en la vela. Llamaremos $\delta_{r}, \delta_{s}, \delta_{b}$ a las deflexiones de los timones (rudder, stern y bow plane respectivamente) en grados y $n$ a la velocidad de giro de la hélice en revoluciones por segundo.

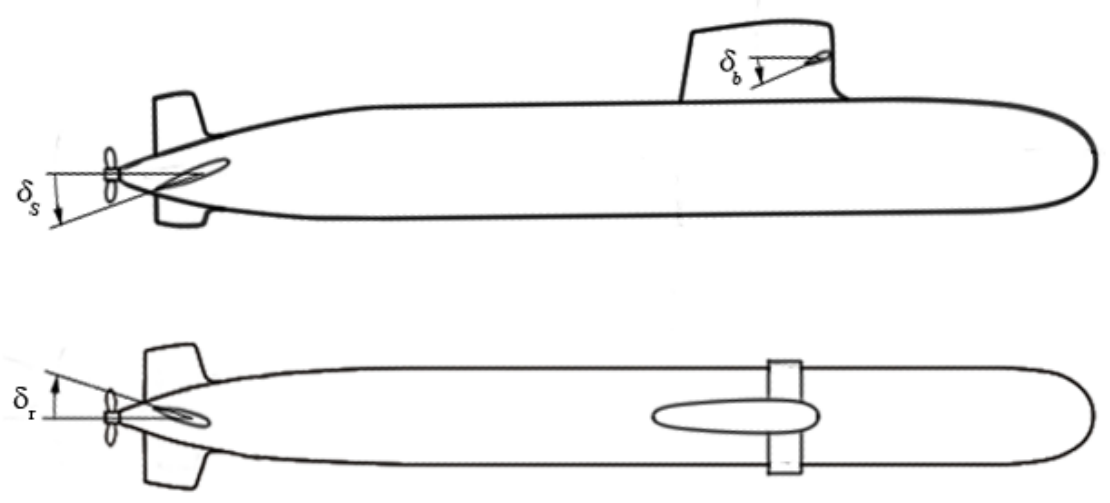

Figura 1.2: Localización de las superficies de control. Vista lateral (arriba) y superior (abajo).

\subsection{Grados de libertad y sistemas de referencia}

Las ecuaciones de Feldman describen el movimiento de un vehículo con 6 grados de libertad (GDL). En efecto, el submarino es libre de desplazarse a lo largo de tres direcciones espaciales así como de rotar alrededor de los tres ejes coordenados. Son necesarias, por tanto, seis coordenadas independientes para determinar su posición y orientación. Las primeras tres coordenadas y sus derivadas respecto al tiempo se corresponden con la posición y traslación en los ejes $x, y, z$, mientras que las últimas tres coordenadas y sus derivadas describen la orientación y movimiento rotacional del vehículo. Usualmente, estas coordenadas se definen, de acuerdo con la nomenclatura adoptada por la SNAME (Society of Naval Architects and Marine Engineers) [SNA50], del modo que se recoge en la Tabla 1.2. Mantenemos la nomenclatura inglesa por no ser los términos castellanos de uso común.

El movimiento de un vehículo submarino se describe habitualmente mediante el uso de dos sistemas de coordenadas: un sistema de referencia móvil, con los ejes fijos en el vehículo, que recibe el nombre de sistema de coordenadas cuerpo, y un sistema de referencia fijo, que recibe el nombre de sistema de coordenadas mundo. La posición y orientación del vehículo se 


\begin{tabular}{|c|l|c|c|c|}
\hline GDL & & $\begin{array}{l}\text { Fuerzas y } \\
\text { momentos }\end{array}$ & $\begin{array}{l}\text { Velocidades } \\
\text { lineales } \\
\text { y angulares }\end{array}$ & $\begin{array}{l}\text { Coordenadas y } \\
\text { ángulos de Euler }\end{array}$ \\
\hline 1 & Movimiento en la dirección $x$ (surge) & $X$ & $u$ & $x$ \\
\hline 2 & Movimiento en la dirección $y$ (sway) & $Y$ & $v$ & $y$ \\
\hline 3 & Movimiento en la dirección $z$ (heave) & $Z$ & $w$ & $z$ \\
\hline 4 & Rotación alrededor del eje $x$ (roll) & $K$ & $p$ & $\phi$ \\
\hline 5 & Rotación alrededor del eje $y($ pitch $)$ & $M$ & $q$ & $\theta$ \\
\hline 6 & Rotación alrededor del eje $z$ (yaw) & $N$ & $r$ & $\psi$ \\
\hline
\end{tabular}

Tabla 1.2: Notación de la SNAME para vehículos submarinos.

describe con respecto al sistema de coordenadas mundo mientras que las velocidades lineales y angulares se refieren al sistema de coordenadas cuerpo. Estas magnitudes se definen, de acuerdo a la notación empleada en [Fos94], como:

$$
\begin{array}{lll}
\eta(t)=\left[\eta_{1}^{T}(t), \eta_{2}^{T}(t)\right], & \eta_{1}(t)=[x(t), y(t), z(t)]^{T}, & \eta_{2}(t)=[\phi(t), \theta(t), \psi(t)]^{T} \\
\nu(t)=\left[\nu_{1}^{T}(t), \nu_{2}^{T}(t)\right], & \nu_{1}(t)=[u(t), v(t), w(t)]^{T}, & \nu_{2}(t)=[p(t), q(t), r(t)]^{T},
\end{array}
$$

donde $a^{T}$ denota la transpuesta del vector $a, \eta_{1}$ denota la posición del vehículo en el sistema mundo, $\eta_{2}$ es la orientación en dicho sistema de referencia, $\nu_{1}$ es el vector de velocidades lineales en el sistema cuerpo (surge velocity, $u$, sway velocity, $v$ y heave velocity, $w$ ), $\mathrm{y}$, por último, $\nu_{2}$ es el vector de velocidades angulares en el sistema cuerpo, que se corresponderán a la tasa de variación de los ángulos de Euler (roll rate, $p$, pitch rate, $q$ y yaw rate, $q$ ). La Figura 1.3 muestra ambos sistemas de referencia así como el significado y criterio de signos adoptado para cada una de las variables.

\subsection{Transformación de coordenadas}

Para estudiar el movimiento de un vehículo submarino es necesario, en primer lugar, ser capaces de calcular las velocidades respecto del sistema fijo conocidas las velocidades en el sistema de referencia móvil, y viceversa. Para conseguir esto, necesitaremos conocer las matrices correspondientes a ambas transformaciones.

El movimiento del vehículo en el sistema de coordenadas fijo vendrá dado por la siguiente transformación de velocidades:

$$
\dot{\eta}_{1}=J_{1}\left(\eta_{2}\right) \cdot \nu_{1}
$$

donde $J_{1}\left(\eta_{2}\right)$ es una matriz de transformación que corresponde a realizar las siguientes operaciones (el orden en que éstas se realizan no es arbitrario): 


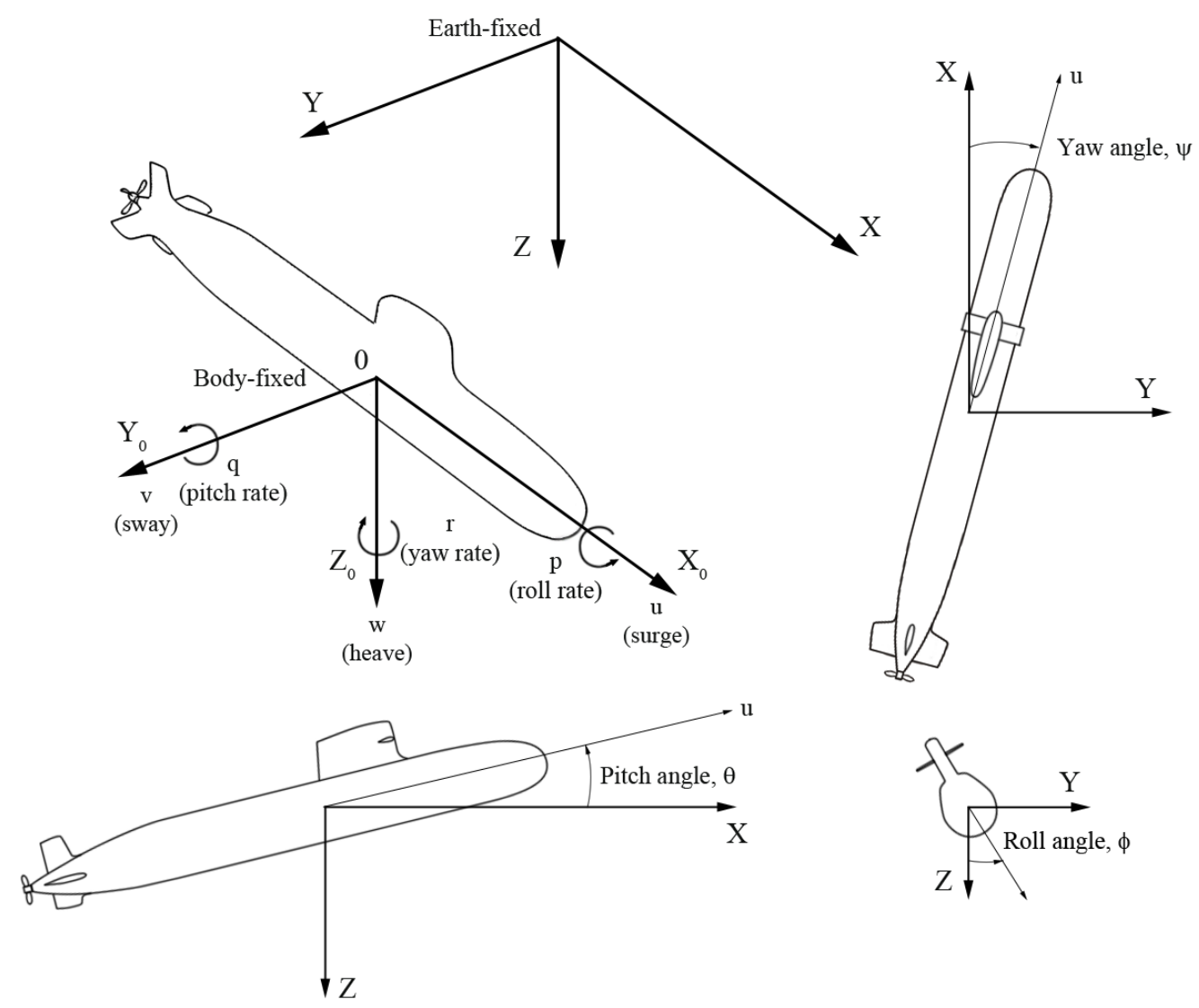

Figura 1.3: Variables y sistemas de referencia.

Sea $X_{3} Y_{3} Z_{3}$ el sistema de coordenadas resultante de trasladar el sistema de coordenadas mundo paralelo a sí mismo hasta que su origen de coordenadas coincida con el origen del sistema de coordenadas cuerpo. Este sistema es rotado un ángulo $\psi$ alrededor del eje $Z_{3}$. El sistema de coordenadas resultante, $X_{2} Y_{2} Z_{2}$, es rotado un ángulo $\theta$ alrededor del eje $Y_{2}$. Por último, el sistema de coordenadas resultante, $X_{1} Y_{1} Z_{1}$, es rotado un ángulo $\phi$ alrededor del eje $X_{1}$, obteniéndose así el sistema de coordenadas cuerpo.

La matriz de transformación resultante es:

$$
J_{1}\left(\eta_{2}\right)=\left[\begin{array}{ccc}
\cos \psi \cos \theta & -\sin \psi \cos \phi+\cos \psi \sin \theta \sin \phi & \sin \psi \sin \theta+\cos \psi \cos \phi \sin \theta \\
\sin \psi \cos \theta & \cos \psi \cos \phi+\sin \phi \sin \theta \sin \psi & -\cos \psi \sin \phi+\sin \theta \sin \psi \cos \phi \\
-\sin \theta & \cos \theta \sin \phi & \cos \theta \cos \phi
\end{array}\right]
$$

que permite obtener las velocidades lineales en el sistema de coordenadas fijo a partir de las velocidades en el sistema de coordenadas móvil. 
De igual modo, las velocidades angulares en el sistema de coordenadas cuerpo, $\nu_{2}=$ $[p, q, r]^{T}$, se relacionan con la variación de los ángulos de Euler, $\dot{\eta}_{2}=[\dot{\phi}, \dot{\theta}, \dot{\psi}]^{T}$, mediante la matriz de transformación $J_{2}\left(\eta_{2}\right)$ :

$$
\dot{\eta}_{2}=J_{2}\left(\eta_{2}\right) \cdot \nu_{2}
$$

con:

$$
J_{2}\left(\eta_{2}\right)=\left[\begin{array}{ccc}
1 & \sin \phi \tan \theta & \cos \phi \tan \theta \\
0 & \cos \phi & -\sin \phi \\
0 & \sin \phi / \cos \theta & \cos \phi / \cos \theta
\end{array}\right]
$$

Una descripción más detallada de ambas transformaciones puede encontrarse en [Fos94].

\subsection{Ecuaciones del sólido rígido}

La dinámica del submarino, como la de cualquier sólido rígido, se obtendrá partiendo de la segunda ley de Newton y las leyes del movimiento relativo. El proceso detallado para la obtención de las ecuaciones del sólido rígido puede consultarse en [Fos94] o en cualquier texto clásico de mecánica. Sea $m$ la masa del vehículo e $I$ el tensor de inercia, la ecuación para el movimiento de traslación será:

$$
m\left(\dot{\overrightarrow{v_{0}}}+\vec{w} \times \overrightarrow{v_{0}}+\dot{\vec{w}} \times \vec{r}_{G}+\vec{w} \times\left(\vec{w} \times \vec{r}_{G}\right)\right)=\overrightarrow{f_{0}}
$$

mientras que para el movimiento de rotación:

$$
I \dot{\vec{w}}+\vec{w} \times(I \vec{w})+m \vec{r}_{G} \times\left(\dot{\vec{v}}_{0}+\vec{w} \times \vec{v}_{0}\right)=\vec{m}_{0} .
$$

Adoptando la notación de [Fos94]:

$$
\begin{aligned}
& \overrightarrow{f_{0}}=\tau_{1}=[X, Y, Z]^{T} \quad \text { Fuerzas externas. } \\
& \vec{m}_{0}=\tau_{2}=[K, M, N]^{T} \quad \text { Momento de las fuerzas externas respecto al origen. } \\
& \vec{v}_{0}=\nu_{1}=[u, v, w]^{T} \quad \text { Velocidades lineales en el sistema móvil. } \\
& \vec{w}=\nu_{2}=[p, q, r]^{T} \quad \text { Velocidades angulares en el sistema móvil. } \\
& \vec{r}_{G} \quad=\left[x_{G}, y_{G}, z_{G}\right]^{T} \quad \text { Centro de gravedad. }
\end{aligned}
$$

y expresando (1.3)-(1.4) en componentes, obtenemos: 


$$
\begin{aligned}
m\left[\dot{u}-v r+w q-x_{G}\left(q^{2}+r^{2}\right)+y_{G}(p q-\dot{r})+z_{G}(p r+\dot{q})\right] & =X \\
m\left[\dot{v}-w p+u r-y_{G}\left(r^{2}+p^{2}\right)+z_{G}(q r-\dot{p})+x_{G}(q p+\dot{r})\right] & =Y \\
m\left[\dot{w}-u q+v p-z_{G}\left(p^{2}+q^{2}\right)+x_{G}(r p-\dot{q})+y_{G}(r q+\dot{p})\right] & =Z \\
I_{x} \dot{p}+\left(I_{z}-I_{y}\right) q r-I_{z x} \dot{r}-I_{z x} p q+I_{y z} r^{2}-I_{y z} q^{2}+I_{x y} p r-I_{x y} \dot{q} & \\
+m y_{G} \dot{w}-m y_{G} u q+m y_{G} v p-m z_{G} \dot{v}+m z_{G} w p-m z_{G} u r & =K \\
I_{y} \dot{q}+\left(I_{x}-I_{z}\right) r p-(\dot{p}+q r) I_{x y}+\left(p^{2}-r^{2}\right) I_{z x}+(q p-\dot{r}) I_{y z} & \\
+m\left[z_{G}(\dot{u}-v r+w q)-x_{G}(\dot{w}-u q+v p)\right] & =M \\
I_{z} \dot{r}+\left(I_{y}-I_{x}\right) p q-(\dot{q}+r p) I_{y z}+\left(q^{2}-p^{2}\right) I_{x y}+(r q-\dot{p}) I_{z x} & \\
+m\left[x_{G}(\dot{v}-w p+u r)-y_{G}(\dot{u}-v r+w q)\right] & =N
\end{aligned}
$$

donde $X, Y, Z, K, M, N$ son, como se dijo anteriormente, las fuerzas y momentos actuantes sobre el vehículo. La forma de evaluar estas fuerzas y momentos se cubrirá brevemente en la siguiente sección.

\subsection{Fuerzas y momentos hidrodinámicos}

Las fuerzas más significativas que actúan sobre el vehículo pueden agruparse en las siguientes categorías:

Peso y flotabilidad: El peso del vehículo actuará sobre el centro de gravedad, $C G$, mientras que la flotabilidad (peso del fluido desplazado) actuará sobre el centro de flotación, $C B$, tal y como muestra la Figura 1.4. Los valores de peso, $W$, y flotabilidad, $B$, serán:

$$
W=g m, \quad B=\rho g \nabla
$$

siendo $g$ la aceleración de la gravedad, $\rho$ la densidad del agua y $\nabla$ el volumen de fluido desplazado por el vehículo.

Sean $\left(x_{G}, y_{G}, z_{G}\right),\left(x_{B}, y_{B}, z_{B}\right)$ las coordenadas en el sistema de referencia móvil de los centros de gravedad y flotación respectivamente, las fuerzas y momentos resultantes serán:

$$
\begin{aligned}
X_{B} & =-(W-B) \sin \theta \\
Y_{B} & =(W-B) \cos \theta \sin \phi \\
Z_{B} & =(W-B) \cos \theta \cos \phi \\
K_{B} & =\left(y_{G} W-y_{B} B\right) \cos \theta \cos \phi-\left(z_{G} W-z_{B} B\right) \cos \theta \sin \phi \\
M_{B} & =-\left(z_{G} W-z_{B} B\right) \sin \phi-\left(x_{G} W-x_{B} B\right) \cos \theta \cos \phi \\
N_{B} & =\left(x_{G} W-x_{B} B\right) \cos \theta \sin \phi+\left(y_{G} W-y_{B} B\right) \sin \phi .
\end{aligned}
$$



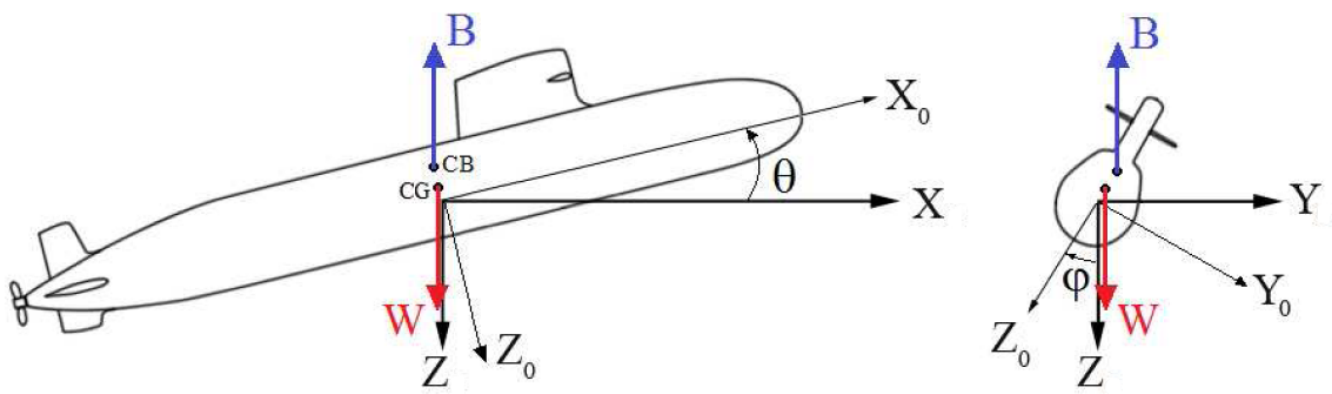

Figura 1.4: Peso y flotabilidad.

Fuerzas de control: Fuerzas empleadas para el control del vehículo, debidas a hélice y timones (ver Sección 1.1). La descripción detallada del los modelos empleados puede encontrarse en [Ova11, Fos94, Car11]. El valor de todas las constantes y parámetros necesarios se recoge en las tablas 1.4 (hélice) y 1.5 (timones).

Masa añadida: La masa añadida se traduce en fuerzas y momentos debidos al campo de presiones alrededor del vehículo. Recibe esta denominación ya que puede entenderse, en cierto modo, como el efecto de la inercia del fluido adyacente al vehículo y que debe ser acelerado por éste en su movimiento. De esta forma a la masa del vehículo se añade, de forma aparente, la del fluido circundante.

Rozamiento: Las fuerzas actuantes sobre la superficie del vehículo son debidas principalmente a dos fenómenos: skin friction, rozamiento debido a la presencia de capas límite (bien laminares o turbulentas) sobre la superficie del submarino; y vortex shedding, desprendimiento de las capas límites debido a la viscosidad del fluido originando pequeños torbellinos.

La obtención de una expresión explícita para estos dos últimos conjuntos de fuerzas no es tarea sencilla. En efecto, su evaluación exacta requeriría la resolución de las ecuaciones de Navier-Stokes alrededor del vehículo, con el enorme coste computacional asociado. Las ecuaciones de Feldman evalúan estas fuerzas como desarrollos en serie de Taylor en función de las variables de estado y sus derivadas. Los términos de estos desarrollos aparecen multiplicados por una serie de coeficientes que reciben el nombre de coeficientes hidrodinámicos y que han de ser determinados para cada vehículo concreto mediante procedimientos semi-empíricos. En el caso del submarino P-650, la determinación de los coeficientes hidrodinámicos se llevó a cabo por parte de la empresa sueca SSPA mediante ensayos con modelo a escala [Bys01a]. Los valores adimensionales de estos coeficientes se recogen en la Tabla 1.5. La Tabla 1.3 recoge los parámetros geométricos del vehículo. 


\subsection{Ecuaciones del movimiento}

La forma final de las ecuaciones del movimiento se compone de un total de 12 ecuaciones diferenciales ordinarias altamente no lineales. Las 6 primeras corresponden a la transformación de coordenadas entre los sistemas de referencia fijo y móvil dada por las matrices (1.1)(1.2) mientras que las 6 restantes corresponden a los equilibrios de fuerzas y momentos proporcionados por (1.5).

\subsubsection{Ecuaciones cinemáticas}

$$
\begin{aligned}
\dot{x}= & u \cos \phi \cos \psi+v(\sin \phi \sin \theta \cos \psi-\cos \phi \sin \psi) \\
& +w(\sin \phi \sin \psi+\cos \phi \sin \theta \cos \psi) \\
\dot{y}= & u \cos \theta \sin \psi+v(\cos \phi \cos \psi+\sin \phi \sin \theta \sin \psi) \\
& +w(\cos \phi \sin \theta \sin \psi-\sin \phi \cos \psi) \\
\dot{z}= & -u \sin \theta+v \cos \theta \sin \phi+w \cos \theta \cos \phi \\
\dot{\phi}= & p+r \cos \phi \tan \theta+q \sin \phi \tan \theta \\
\dot{\theta}= & q \cos \phi-r \sin \phi \\
\dot{\psi}= & \frac{r \cos \phi+q \sin \phi}{\cos \theta}
\end{aligned}
$$

\subsubsection{Ecuaciones dinámicas}

\section{Axial Force Equation:}

$$
\begin{aligned}
& m\left[\dot{u}-v r+w q-x_{G}\left(q^{2}+r^{2}\right)+y_{G}(p q-\dot{r})+z_{G}(p r+\dot{q})\right] \\
& =\frac{\rho}{2} l^{4}\left[X_{q q}^{\prime} q^{2}+X_{r r}^{\prime} r^{2}+X_{r p}^{\prime} r p+X_{q|q|}^{\prime} q|q|\right]+\frac{\rho}{2} l^{3}\left[X_{\dot{u}}^{\prime} \dot{u}+X_{v r}^{\prime} v r+X_{w q}^{\prime} w q\right] \\
& \quad+\frac{\rho}{2} l^{2}\left[X_{u u}^{\prime} u^{2}+X_{v v}^{\prime} v^{2}+X_{w w}^{\prime} w^{2}+X_{w|w|}^{\prime} w|w|\right]+\rho T_{p}\left(1-t_{p}\right) \\
& \quad+\frac{\rho}{2} l^{2}\left[X_{\delta_{r} \delta_{r}}^{\prime} u^{2} \delta_{r}^{2}+X_{\delta_{s} \delta_{s}}^{\prime} u^{2} \delta_{s}^{2}+X_{\delta_{b} \delta_{b}}^{\prime} u^{2} \delta_{b}^{2}\right]-(W-B) \sin \theta \\
& \operatorname{con} T_{p}=K_{T 0} n^{2} D^{4}+K_{T J}\left(1-w_{f}\right) n D^{3} u+K_{T J 2}\left(1-w_{f}\right)^{2} D^{2} u^{2} .
\end{aligned}
$$




\section{Lateral Force Equation:}

$$
\begin{aligned}
m & {\left[\dot{v}-w p+u r-y_{G}\left(r^{2}+p^{2}\right)+z_{G}(q r-\dot{p})+x_{G}(q p+\dot{r})\right] } \\
= & \frac{\rho}{2} l^{4}\left[Y_{\dot{r}}^{\prime} \dot{r}+Y_{\dot{p}}^{\prime} \dot{p}+Y_{r|r|}^{\prime} r|r|+Y_{p q}^{\prime} p q\right]+\frac{\rho}{2} l^{3}\left[Y_{r}^{\prime} u r+Y_{p}^{\prime} u p+Y_{\dot{v}}^{\prime} \dot{v}+Y_{w p}^{\prime} w p\right] \\
& +\frac{\rho}{2} l^{2}\left[Y_{*}^{\prime} u^{2}+Y_{v}^{\prime} u v+Y_{v|v| N}^{\prime} v\left|\left(v^{2}+w^{2}\right)^{\frac{1}{2}}\right|\right] \\
& +\frac{\rho}{2} l^{2}\left[Y_{\delta_{r}}^{\prime} u^{2} \delta_{r}+Y_{\delta_{r} \eta}^{\prime} u^{2} \delta_{r}\left(\eta-\frac{1}{C}\right) C\right]+\frac{\rho}{2} l^{2} Y_{v w N}^{\prime} v w+(W-B) \cos \theta \sin \phi
\end{aligned}
$$

\section{Normal Force Equation:}

$$
\begin{aligned}
m & {\left[\dot{w}-u q+v p-z_{G}\left(p^{2}+q^{2}\right)+x_{G}(r p-\dot{q})+y_{G}(r q+\dot{p})\right] } \\
= & \frac{\rho}{2} l^{4}\left[Z_{\dot{q}}^{\prime} \dot{q}+Z_{q|q|}^{\prime} q|q|+Z_{r r}^{\prime} r^{2}\right]+\frac{\rho}{2} l^{3}\left[Z_{\dot{w}}^{\prime} \dot{w}+Z_{q}^{\prime} u q+Z_{v p}^{\prime} v p+Z_{v r}^{\prime} v r\right] \\
& +\frac{\rho}{2} l^{2}\left[Z_{*}^{\prime} u^{2}+Z_{w}^{\prime} u w+Z_{v v}^{\prime} v^{2}\right]+\frac{\rho}{2} l^{2}\left[Z_{|w|}^{\prime} u|w|+Z_{w w N}^{\prime}|w|\left(v^{2}+w^{2}\right)^{\frac{1}{2}}\right] \\
& +\frac{\rho}{2} l^{2}\left[Z_{\delta_{s}}^{\prime} u^{2} \delta_{s}+Z_{\delta_{b}}^{\prime} u^{2} \delta_{b}+Z_{\delta_{s} \eta}^{\prime} u^{2} \delta_{s}\left(\eta-\frac{1}{C}\right) C\right] \\
& +(W-B) \cos \theta \cos \phi
\end{aligned}
$$

\section{Rolling Moment Equation:}

$$
\begin{aligned}
& I_{x} \dot{p}+\left(I_{z}-I_{y}\right) q r-I_{z x} \dot{r}-I_{z x} p q+I_{y z} r^{2}-I_{y z} q^{2}+I_{x y} p r-I_{x y} \dot{q} \\
& +m\left[y_{G}(\dot{w}-u q+v p)-z_{G}(\dot{v}+w p-u r)\right] \\
& =\frac{\rho}{2} l^{5}\left[K_{\dot{p}}^{\prime} \dot{p}+K_{\dot{r}}^{\prime} \dot{r}+K_{q r}^{\prime} q r+K_{p|p|}^{\prime} p|p|+K_{r|r|}^{\prime} r|r|\right] \\
& \quad+\frac{\rho}{2} l^{4}\left[K_{p}^{\prime} u p+K_{r}^{\prime} u r+K_{\dot{v}}^{\prime} \dot{v}+K_{w p}^{\prime} w p\right] \\
& \quad+\frac{\rho}{2} l^{3}\left[K_{*}^{\prime} u^{2}+K_{v}^{\prime} u v+K_{v|v|}^{\prime} v|v|+K_{\delta_{r}}^{\prime} u^{2} \delta_{r}\right]-\rho Q_{p} \\
& \quad+\left(y_{G} W-y_{B} B\right) \cos \theta \cos \phi-\left(z_{G} W-z_{B} B\right) \cos \theta \sin \phi
\end{aligned}
$$

$\operatorname{con} Q_{p}=K_{Q 0} n^{2} D^{5}+K_{Q J}\left(1-w_{f}\right) n D^{4} u+K_{Q J 2}\left(1-w_{f}\right)^{2} D^{3} u^{2}$ 


\section{Pitching Moment Equation:}

$$
\begin{aligned}
& I_{y} \dot{q}+\left(I_{x}-I_{z}\right) r p-(\dot{p}+q r) I_{x y}+\left(p^{2}-r^{2}\right) I_{z x}+(q p-\dot{r}) I_{y z} \\
& +m\left[z_{G}(\dot{u}-v r+w q)-x_{G}(\dot{w}-u q+v p)\right] \\
& =\frac{\rho}{2} l^{5}\left[M_{\dot{q}}^{\prime} \dot{q}+M_{r p}^{\prime} r p+M_{q|q|}^{\prime} q|q|+M_{r r}^{\prime} r^{2}\right]+\frac{\rho}{2} l^{4}\left[M_{\dot{w}}^{\prime} \dot{w}+M_{q}^{\prime} u q+M_{v r}^{\prime} v r\right] \\
& \quad+\frac{\rho}{2} l^{3}\left[M_{*}^{\prime} u^{2}+M_{w}^{\prime} u w+M_{v v}^{\prime} v^{2}+M_{w|w| N}^{\prime} w\left|\left(v^{2}+w^{2}\right)^{\frac{1}{2}}\right|\right] \\
& \quad+\frac{\rho}{2} l^{3}\left[M_{v w}^{\prime} v w+M_{|w|}^{\prime} u|w|+M_{w w}^{\prime}\left|w\left(v^{2}+w^{2}\right)^{\frac{1}{2}}\right|\right] \\
& \quad+\frac{\rho}{2} l^{3}\left[M_{\delta_{s}}^{\prime} u^{2} \delta_{s}+M_{\delta_{b}}^{\prime} u^{2} \delta_{b}+M_{\delta_{s} \eta}^{\prime} u^{2} \delta_{s}\left(\eta-\frac{1}{C}\right) C\right] \\
& \quad-\left(x_{G} W-x_{B} B\right) \cos \theta \cos \phi-\left(z_{G} W-z_{B} B\right) \sin \theta
\end{aligned}
$$

Yawing Moment Equation:

$$
\begin{aligned}
I_{z} \dot{r} & +\left(I_{y}-I_{x}\right) p q-(\dot{q}+r p) I_{y z}+\left(q^{2}-p^{2}\right) I_{x y}+(r q-\dot{p}) I_{z x} \\
+ & m\left[x_{G}(\dot{v}-w p+u r)-y_{G}(\dot{u}-v r+w q)\right] \\
= & \frac{\rho}{2} l^{5}\left[N_{\dot{r}}^{\prime} \dot{r}+N_{r|r|}^{\prime} r|r|+N_{\dot{p}}^{\prime} \dot{p}+N_{p q}^{\prime} p q\right]+\frac{\rho}{2} l^{4}\left[N_{p}^{\prime} u p+N_{r}^{\prime} u r+N_{\dot{v}}^{\prime} \dot{v}\right] \\
& +\frac{\rho}{2} l^{3}\left[N_{*}^{\prime} u^{2}+N_{v}^{\prime} u v+N_{v|v| N}^{\prime} v\left|\left(v^{2}+w^{2}\right)^{\frac{1}{2}}\right|\right]+\frac{\rho}{2} l^{3} N_{v w N}^{\prime} v w \\
& +\frac{\rho}{2} l^{3}\left[N_{\delta_{r}}^{\prime} u^{2} \delta_{r}+N_{\delta_{r} \eta}^{\prime} u^{2} \delta_{r}\left(\eta-\frac{1}{C}\right) C\right] \\
& +\left(x_{G} W-x_{B} B\right) \cos \theta \sin \phi+\left(y_{G} W-y_{B} B\right) \sin \theta
\end{aligned}
$$

\begin{tabular}{|l|c||l|c||l|c|}
\hline$l$ & $67 \mathrm{~m}$ & $m$ & $2352 \cdot 10^{3} \mathrm{~kg}$ & $B$ & $23073 \cdot 10^{3} \mathrm{~N}$ \\
\hline$x_{G}$ & $0 \mathrm{~m}$ & $y_{G}$ & $0 \mathrm{~m}$ & $z_{G}$ & $-0,264 \mathrm{~m}$ \\
\hline$x_{B}$ & $0 \mathrm{~m}$ & $y_{B}$ & $0 \mathrm{~m}$ & $z_{B}$ & $-0,595 \mathrm{~m}$ \\
\hline$I_{x}$ & $16390 \cdot 10^{3} \mathrm{kgm}^{2}$ & $I_{y}$ & $659770 \cdot 10^{3} \mathrm{kgm}^{2}$ & $I_{z}$ & $659770 \cdot 10^{3} \mathrm{kgm}^{2}$ \\
\hline$I_{x y}$ & $0 \mathrm{kgm}^{2}$ & $I_{x z}$ & $0 \mathrm{kgm}^{2}$ & $I_{y z}$ & $0 \mathrm{kgm}^{2}$ \\
\hline
\end{tabular}

Tabla 1.3: Parámetros geométricos del submarino P-650. 


\begin{tabular}{|l|c||l|c|}
\hline$K_{T 0}$ & 0,5382 & $K_{T J}$ & $-0,3811$ \\
\hline$K_{T J 2}$ & $-0,1645$ & $K_{T J 3}$ & 0 \\
\hline$K_{T J 4}$ & 0 & $\eta$ & 1,09431 \\
\hline$C$ & 0,8976 & $K_{Q 0}$ & 0,0701 \\
\hline$K_{Q J}$ & $-0,0273$ & $K_{Q J 2}$ & $-0,0345$ \\
\hline$K_{Q J 3}$ & 0 & $K_{Q J 4}$ & 0 \\
\hline$t_{p}$ & 0,148 & $w_{f}$ & 0,344 \\
\hline$D$ & $3,821 \mathrm{~m}$ & & \\
\hline
\end{tabular}

Tabla 1.4: Coeficientes del modelo de la hélice. 


\begin{tabular}{|c|c|c|c|c|c|}
\hline$X_{\dot{u}}^{\prime}$ & $-0,46 \cdot 10^{-3}$ & $Y_{\dot{p}}^{\prime}$ & $-0,3 \cdot 10^{-3}$ & $Z_{\dot{q}}^{\prime}$ & $-0,07 \cdot 10^{-3}$ \\
\hline$X_{r p}^{\prime}$ & $0,6 \cdot 10^{-3}$ & $Y_{\dot{r}}^{\prime}$ & $-0,83 \cdot 10^{-3}$ & $Z_{\dot{w}}^{\prime}$ & $-14,40 \cdot 10^{-3}$ \\
\hline$X_{q q}^{\prime}$ & $1,42 \cdot 10^{-3}$ & $Y_{\dot{v}}^{\prime}$ & $-18,37 \cdot 10^{-3}$ & $Z_{v p}^{\prime}$ & $-18,37 \cdot 10^{-3}$ \\
\hline$X_{q|q|}^{\prime}$ & 0 & $Y_{p}^{\prime}$ & $-3,05 \cdot 10^{-3}$ & $Z_{q}^{\prime}$ & $-6,99 \cdot 10^{-3}$ \\
\hline$X_{r r}^{\prime}$ & $2,08 \cdot 10^{-3}$ & $Y_{p q}^{\prime}$ & $-0,07 \cdot 10^{-3}$ & $Z_{q|q|}^{\prime}$ & $-3,98 \cdot 10^{-3}$ \\
\hline$X_{v r}^{\prime}$ & $22,37 \cdot 10^{-3}$ & $Y_{w p}^{\prime}$ & $14,40 \cdot 10^{-3}$ & $Z_{r r}^{\prime}$ & $-3,96 \cdot 10^{-3}$ \\
\hline$X_{u u}^{\prime}$ & $-1,124 \cdot 10^{-3}$ & $Y_{r}^{\prime}$ & $7 \cdot 10^{-3}$ & $Z_{v r}^{\prime}$ & $-45,13 \cdot 10^{-3}$ \\
\hline$X_{v v}^{\prime}$ & $17,46 \cdot 10^{-3}$ & $Y_{r|r|}^{\prime}$ & $-4,65 \cdot 10^{-3}$ & $Z_{v v}^{\prime}$ & 0,185 \\
\hline$X_{w w}^{\prime}$ & $7,75 \cdot 10^{-3}$ & $Y_{v}^{\prime}$ & $-61,36 \cdot 10^{-3}$ & $Z_{w}^{\prime}$ & $-20,28 \cdot 10^{-3}$ \\
\hline$X_{w|w|}^{\prime}$ & 0 & $Y_{v|v|}^{\prime}$ & $-28,14 \cdot 10^{-3}$ & $Z_{|w|}^{\prime}$ & $-1,99 \cdot 10^{-3}$ \\
\hline$X_{w q}^{\prime}$ & $-13,16 \cdot 10^{-3}$ & $Y_{\delta_{r}}^{\prime}$ & $-0,83 \cdot 10^{-3}$ & $Z_{w w}^{\prime}$ & $20,00 \cdot 10^{-3}$ \\
\hline$X_{w|q|}^{\prime}$ & 0 & $Y_{\delta_{r} \eta}^{\prime}$ & $0,67 \cdot 10^{-3}$ & $Z_{\delta_{s}}^{\prime}$ & $-5,12 \cdot 10^{-3}$ \\
\hline$X_{\delta_{r} \delta_{r}}^{\prime}$ & $-3,90 \cdot 10^{-3}$ & $Y_{*}^{\prime}$ & 0 & $Z_{\delta_{s} \eta}^{\prime}$ & $-0,45 \cdot 10^{-3}$ \\
\hline$X_{\delta_{s} \delta_{s}}^{\prime}$ & $-1,19 \cdot 10^{-3}$ & $Y_{v w N}^{\prime}$ & 0 & $Z_{\delta_{b}}^{\prime}$ & $-5,12 \cdot 10^{-3}$ \\
\hline$X_{\delta_{b} \delta_{b}}^{\prime}$ & $-2,99 \cdot 10^{-3}$ & $Y_{v|v| N}^{\prime}$ & $-0,1162$ & $Z_{*}^{\prime}$ & $-0,3 \cdot 10^{-3}$ \\
\hline$X_{\delta_{b} \delta_{b}}^{\prime}$ & $-2,99 \cdot 10^{-3}$ & $Y_{v|v| N}^{\prime}$ & $-0,1162$ & $Z_{*}^{\prime}$ & $-0,3 \cdot 10^{-3}$ \\
\hline$M_{\dot{q}}^{\prime}$ & $-0,98 \cdot 10^{-3}$ & $N_{\dot{p}}^{\prime}$ & $-0,03 \cdot 10^{-3}$ & $Z_{w|w| R}^{\prime}$ & 0 \\
\hline$M_{\dot{w}}^{\prime}$ & $-1,39 \cdot 10^{-3}$ & $N_{\dot{r}}^{\prime}$ & $-1,20 \cdot 10^{-3}$ & $K_{\dot{p}}^{\prime}$ & $-0,07 \cdot 10^{-3}$ \\
\hline$M_{r p}^{\prime}$ & $1,12 \cdot 10^{-3}$ & $N_{\dot{v}}^{\prime}$ & $0,68 \cdot 10^{-3}$ & $K_{\dot{r}}^{\prime}$ & $-0,05 \cdot 10^{-3}$ \\
\hline$M_{q}^{\prime}$ & $-3,89 \cdot 10^{-3}$ & $N_{p}^{\prime}$ & $-0,68 \cdot 10^{-3}$ & $K_{\dot{v}}^{\prime}$ & $-0,60 \cdot 10^{-3}$ \\
\hline$M_{q|q|}^{\prime}$ & $-3,03 \cdot 10^{-3}$ & $N_{p q}^{\prime}$ & $-0,91 \cdot 10^{-3}$ & $K_{p}^{\prime}$ & $-0,62 \cdot 10^{-3}$ \\
\hline$M_{r r}^{\prime}$ & $-1,19 \cdot 10^{-3}$ & $N_{r}^{\prime}$ & $-4,83 \cdot 10^{-3}$ & $K_{p|p|}^{\prime}$ & $-0,30 \cdot 10^{-3}$ \\
\hline$M_{v r}^{\prime}$ & $-15,73 \cdot 10^{-3}$ & $N_{r|r|}^{\prime}$ & $2,09 \cdot 10^{-3}$ & $K_{w p}^{\prime}$ & $0,30 \cdot 10^{-3}$ \\
\hline$M_{v v}^{\prime}$ & $34,61 \cdot 10^{-3}$ & $N_{v}^{\prime}$ & $-18,64 \cdot 10^{-3}$ & $K_{q r}^{\prime}$ & $-0,21 \cdot 10^{-3}$ \\
\hline$M_{w}^{\prime}$ & $4,78 \cdot 10^{-3}$ & $N_{v|v| R}^{\prime}$ & $18,50 \cdot 10^{-3}$ & $K_{r}^{\prime}$ & $0,26 \cdot 10^{-3}$ \\
\hline$M_{|w|}^{\prime}$ & $-0,36 \cdot 10^{-3}$ & $N_{\delta_{r}}^{\prime}$ & $-3,53 \cdot 10^{-3}$ & $K_{r|r|}^{\prime}$ & $-0,19 \cdot 10^{-3}$ \\
\hline$M_{w|w| R}^{\prime}$ & $-6,74 \cdot 10^{-3}$ & $N_{\delta_{r} \eta}^{\prime}$ & $-0,34 \cdot 10^{-3}$ & $K_{v}^{\prime}$ & $-2,87 \cdot 10^{-3}$ \\
\hline$M_{w w}^{\prime}$ & $0,45 \cdot 10^{-3}$ & $N_{*}^{\prime}$ & 0 & $K_{v|v|}^{\prime}$ & $-2,14 \cdot 10^{-3}$ \\
\hline$M_{\delta_{s}}^{\prime}$ & $-2,31 \cdot 10^{-3}$ & $N_{v w N}^{\prime}$ & $-0,303$ & $K_{\delta_{r}}^{\prime}$ & $0,13 \cdot 10^{-3}$ \\
\hline$M_{\delta_{s} \eta}^{\prime}$ & $-0,38 \cdot 10^{-3}$ & & & $K_{*}^{\prime}$ & 0 \\
\hline$M_{\delta_{b}}^{\prime}$ & $0,94 \cdot 10^{-3}$ & & & & \\
\hline$M_{*}^{\prime}$ & $0,02 \cdot 10^{-3}$ & & & & \\
\hline
\end{tabular}

Tabla 1.5: Coeficientes hidrodinámicos adimensionales. 



\section{CAPÍTULO 2}

\section{Perturbaciones externas}

Como anticipamos en el capítulo anterior, algunas situaciones particulares requieren la revisión de parte de las hipótesis de partida para la obtención de las ecuaciones del movimiento. A lo largo de este capítulo ampliaremos dichas ecuaciones para incorporar el efecto de perturbaciones externas, en concreto la acción del oleaje sobre el vehículo y los cambios en la flotabilidad producidos por variaciones en las propiedades del agua o la compresión del vehículo por efecto de la presión exterior. El modelado de los esfuerzos producidos por el oleaje se cubrirá en la Sección 2.1 mientras que las variaciones en la flotabilidad se tratarán en la Sección 2.2.

\subsection{Fuerzas inducidas por el oleaje}

Puesto que su importancia disminuye al aumentar la cota, considerándose generalmente despreciables para cotas superiores a los 50-60 m, los esfuerzos producidos sobre el submarino por las olas superficiales son habitualmente ignorados. Cuando el vehículo opera en las proximidades de la superficie, sin embargo, estos esfuerzos pueden alcanzar magnitudes considerables llegando incluso a comprometer la estabilidad del vehículo. En estas circunstancias resulta imprescindible tener en cuenta el efecto que el oleaje ejerce sobre el submarino.

La Sección 2.1.1 resume las técnicas más extendidas para modelar el movimiento de la superficie del océano. El cálculo de los esfuerzos originados sobre el submarino por este movimiento se trata en la Sección 2.1.2. 


\subsubsection{Mar irregular}

El comportamiento de la superficie oceánica es aleatorio en el tiempo y el espacio. En ingeniería naval, al mar de estas características se le conoce comúnmente como mar irregular. El método más extendido para modelar el comportamiento de un mar irregular consiste en suponer que éste puede ser descrito mediante la superposición de ondas senoidales regulares. La descripción de esta metodología puede encontrarse en [Fos94] o con mayor detalle y un completo análisis estadístico en [P0́5].

Llamamos onda regular a una onda senoidal que se propaga por la superficie del fluido. Si suponemos que avanza en la dirección positiva del eje $x$, la elevación de la superficie del océano, $\zeta(x, t)$, viene dada por

$$
\zeta(x, t)=A \cos (w t-k x+\phi)
$$

donde $A$ es la amplitud, $w$ es la frecuencia angular, $k$ es el número de onda y $\phi$ es la fase inicial. La Figura 2.1 muestra las principales características de una onda regular.
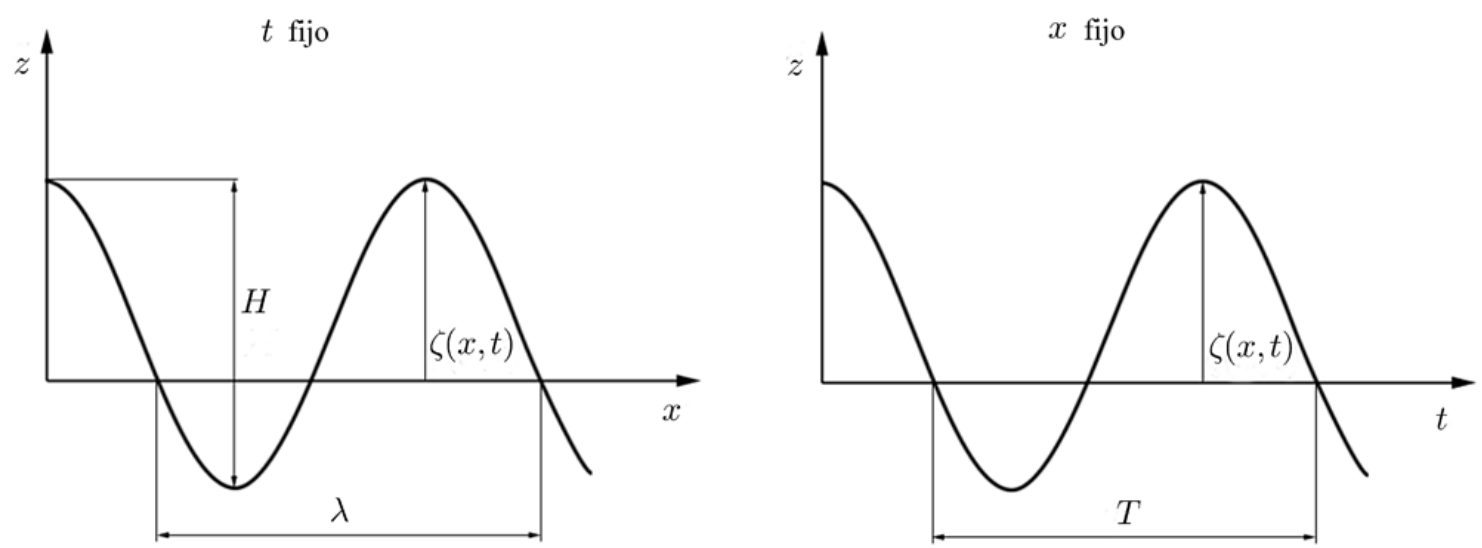

Figura 2.1: Parámetros principales de una onda regular.

La altura pico-pico es $H=2 A, \lambda$ es la longitud de onda, $T$ es el periodo, $T=\frac{2 \pi}{\omega}$, y la velocidad de propagación es $c=\frac{\lambda}{T}=\omega k$. La relación entre la frecuencia y el número de onda es

$$
\omega^{2}=k g \tanh (k d)
$$

donde $d$ es la profundidad del agua. Para profundidad infinita, es decir $\frac{d}{\lambda}>1 / 2$, esta relación se reduce a

$$
\omega^{2}=k g
$$

De forma similar, la elevación producida por un mar irregular propagándose en la dirección 
positiva del eje $x$ puede ser modelada como la superposición de $N$ ondas regulares:

$$
\zeta(x, t)=\sum_{i=1}^{N} A_{i} \cos \left(w_{i} t-k_{i} x+\phi_{i}\right) .
$$

\section{Espectro de energía}

El modo en que la energía se distribuye en las diferentes frecuencias es descrito por el espectro de energía. Existen diversas formulaciones para el espectro de energía [Fos94, Pó5] basadas en largas series de observaciones llevadas a cabo en diferentes puntos del océano. La $15^{\mathrm{a}}$ International Towing Tank Conference (ITTC, 1978) recomendó el uso del espectro de Pierson-Moskowitz modificado:

$$
S(\omega)=A \cdot B \cdot \omega^{-5} \cdot \mathrm{e}^{-B \omega^{-4}}
$$

con

$$
\begin{aligned}
A & =0,25 \cdot H_{1 / 3}^{2} \\
B & =\left(\frac{2 \pi \cdot 0,752}{T_{z}}\right)^{4}
\end{aligned}
$$

Este modelo depende de dos parámetros:

Altura de ola significativa, $H_{1 / 3}$ : Altura media de las olas pertenecientes al tercio de mayor tamaño.

Periodo de paso por cero, $T_{z}$ : Periodo medio para pasos sucesivos por cero en un mismo sentido.

El valor de estos parámetros depende del estado de la mar considerado (Tabla 2.1). En el caso del Mar Mediterráneo, los valores para estados de mar entre 3 y 6 están recogidos en la Tabla 2.2.

La Figura 2.2 muestra la función de densidad espectral (2.5) para los estados de mar 3-6 según los datos de la Tabla 2.2.

La amplitud $A_{i}$ de la $i$-ésima componente de (2.4) se relaciona con la función de densidad espectral $S(\omega)$ en la forma

$$
A_{i}=\sqrt{2 S\left(\omega_{i}\right) \Delta \omega}
$$

donde $\Delta \omega$ es la amplitud de cada uno de los subintervalos en los que se divide el espectro de frecuencias,

$$
\Delta \omega=\frac{\omega_{\max }-\omega_{\min }}{N}
$$




\begin{tabular}{|c|l|c|}
\hline Código & Descripción & Altura de ola, $H_{1 / 3}[\mathrm{~m}]$ \\
\hline 0 & Mar llana (glassy) & 0 \\
\hline 1 & Mar rizada (rippled) & $0-0,1$ \\
\hline 2 & Marejadilla (smooth) & $0,1-0,5$ \\
\hline 3 & Marejada (slight) & $0,5-1,25$ \\
\hline 4 & Fuerte marejada (moderate) & $1,25-2,5$ \\
\hline 5 & Gruesa (rough) & $2,5-4,0$ \\
\hline 6 & Muy gruesa (very rough) & $4,0-6,0$ \\
\hline 7 & Arbolada (high) & $6,0-9,0$ \\
\hline 8 & Montañosa (very high) & $9,0-14,0$ \\
\hline 9 & Enorme (phenomenal) & $14,0-$ \\
\hline
\end{tabular}

Tabla 2.1: Escala Douglas para la descripción de los diferentes estados de mar.

\begin{tabular}{|c|c|c|}
\hline Estado de mar & Altura de ola, $H_{1 / 3}[\mathrm{~m}]$ & Periodo, $T_{z}[\mathrm{~s}]$ \\
\hline 3 & 0,88 & 5,2 \\
\hline 4 & 1,88 & 5,7 \\
\hline 5 & 3,25 & 6,2 \\
\hline 6 & 5,00 & 6,6 \\
\hline
\end{tabular}

Tabla 2.2: Valores de $H_{1 / 3}$ y $T_{z}$ para diferentes estados de mar en el Mediterráneo. Tabla tomada de [Bys01b].

\section{Frecuencia de encuentro}

La expresión (2.4) es válida para un observador en reposo. Si el observador se mueve con velocidad $U$, la frecuencia con la que ve las olas se verá modificada en función de la velocidad $U$ y del ángulo relativo entre esta velocidad y la de propagación de las olas, $\chi$ (Figura 2.3). La frecuencia de encuentro es:

$$
\omega_{e}=\omega-\frac{\omega^{2}}{g} U \cos \chi
$$

Puesto que la frecuencia de las olas encontradas es diferente, el espectro de energía debe desplazarse para cubrir un rango diferente de frecuencias. Dado que el área bajo el espectro de frecuencias en el intervalo $\Delta \omega$ representa la energía contenida en dicha banda de frecuencias, y dicha energía no se ve modificada por un cambio en el sistema de referencia, resulta evidente que esta energía debe ser igual a la contenida en el correspondiente intervalo $\Delta \omega_{e}$. Por tanto, debe verificarse la relación

$$
S(\omega) \Delta \omega=S\left(\omega_{e}\right) \Delta \omega_{e}
$$




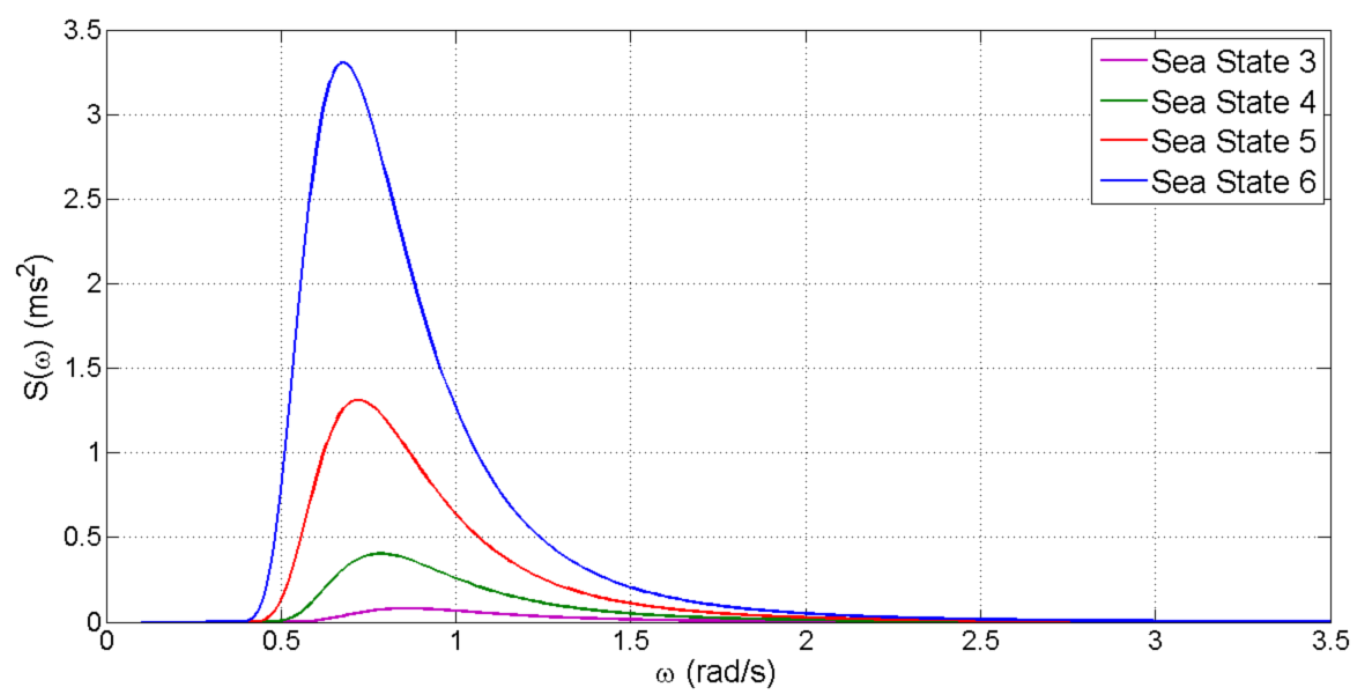

Figura 2.2: Distribución de la energía a lo largo del espectro de frecuencias para diferentes estados de mar según Tabla 1.3.

y si $\Delta \omega$ y $\Delta \omega_{e}$ se hacen infinitesimales

$$
S\left(\omega_{e}\right)=S(\omega) \frac{\mathrm{d} \omega}{\mathrm{d} \omega_{e}}=S(\omega) \frac{g}{g-2 \omega U \cos \chi} .
$$

De (2.9) se desprende que es indiferente emplear las frecuencias $\omega \mathrm{u} \omega_{e}$ para calcular las amplitudes $A_{i}$ mediante (2.6).

Nótese, por otra parte, que ni $\lambda_{i}$ ni $k_{i}$ se ven modificadas por el cambio de sistema de referencia.

La expresión para la elevación toma ahora la forma

$$
\zeta(x, y, t)=\sum_{i=1}^{N} A_{i} \cos \left(w_{e, i} t-k_{i}(x \cos \chi-y \sin \chi)+\phi_{i}\right) .
$$

\section{Long crested frente a short crested}

El desarrollo anterior supone que las olas se propagan en una única dirección, lo que da lugar a lo que se conoce como long crested waves (Figura 2.4, izquierda). En la realidad las olas se propagan en diferentes direcciones. Este hecho puede tenerse en cuenta introduciendo una función de dispersión (véase [P0́5]) obteniéndose lo que se conoce como short crested waves (Figura 2.4, derecha). La Figura 2.4 muestra la simulación de un estado de mar 3 mediante ambos modelos y la función de dispersión propuesta en [Pó5] con $N=100, M=10$ 


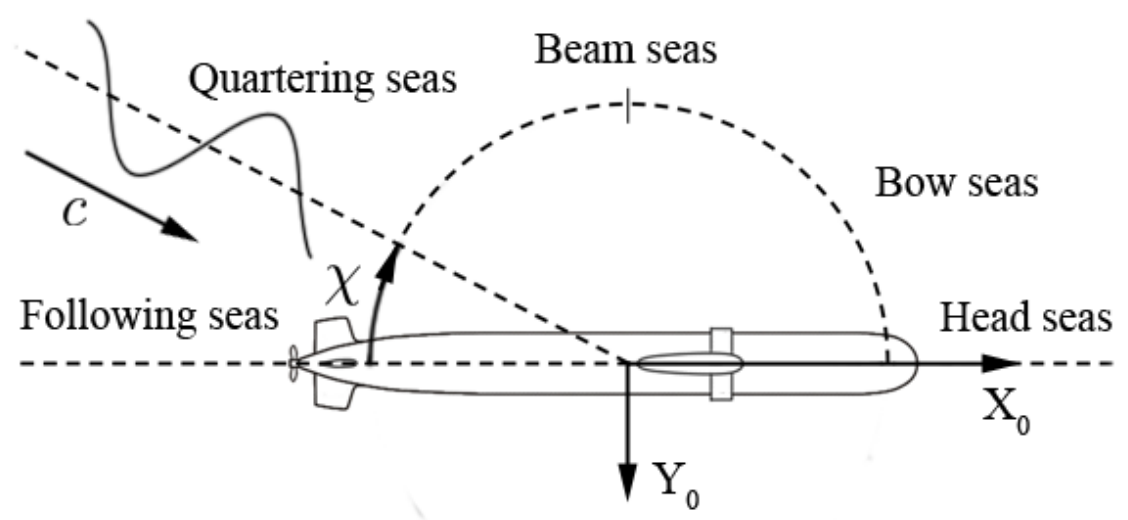

Figura 2.3: Ángulo relativo entre vehículo y oleaje.

y $n=1$ (el significado de estos parámetros puede encontrarse en [Pó5]). Como puede verse, el modelo short crested proporciona resultados más realistas. Sin embargo, en la Figura 2.5 podemos ver que, para un punto fijo, la evolución a lo largo del tiempo obtenida con ambos modelos presenta un comportamiento cualitativo muy similar. Por este motivo, es usual emplear el modelo long crested, notablemente más sencillo, al realizar simulaciones numéricas. Ésta será la solución adoptada a lo largo de este trabajo.
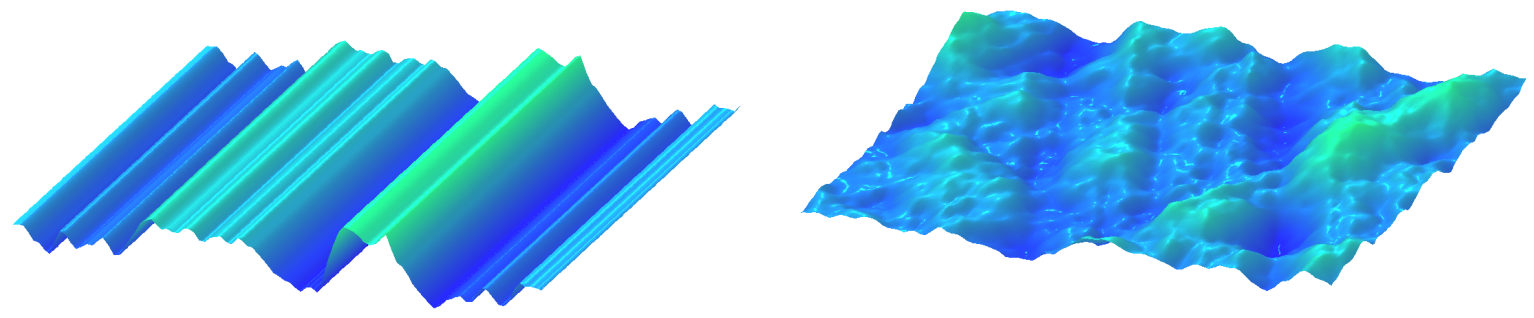

Figura 2.4: Simulación de un estado de mar 3 en un área de 200 x 200 m empleando el modelo long crested (izquierda) y short crested (derecha). 

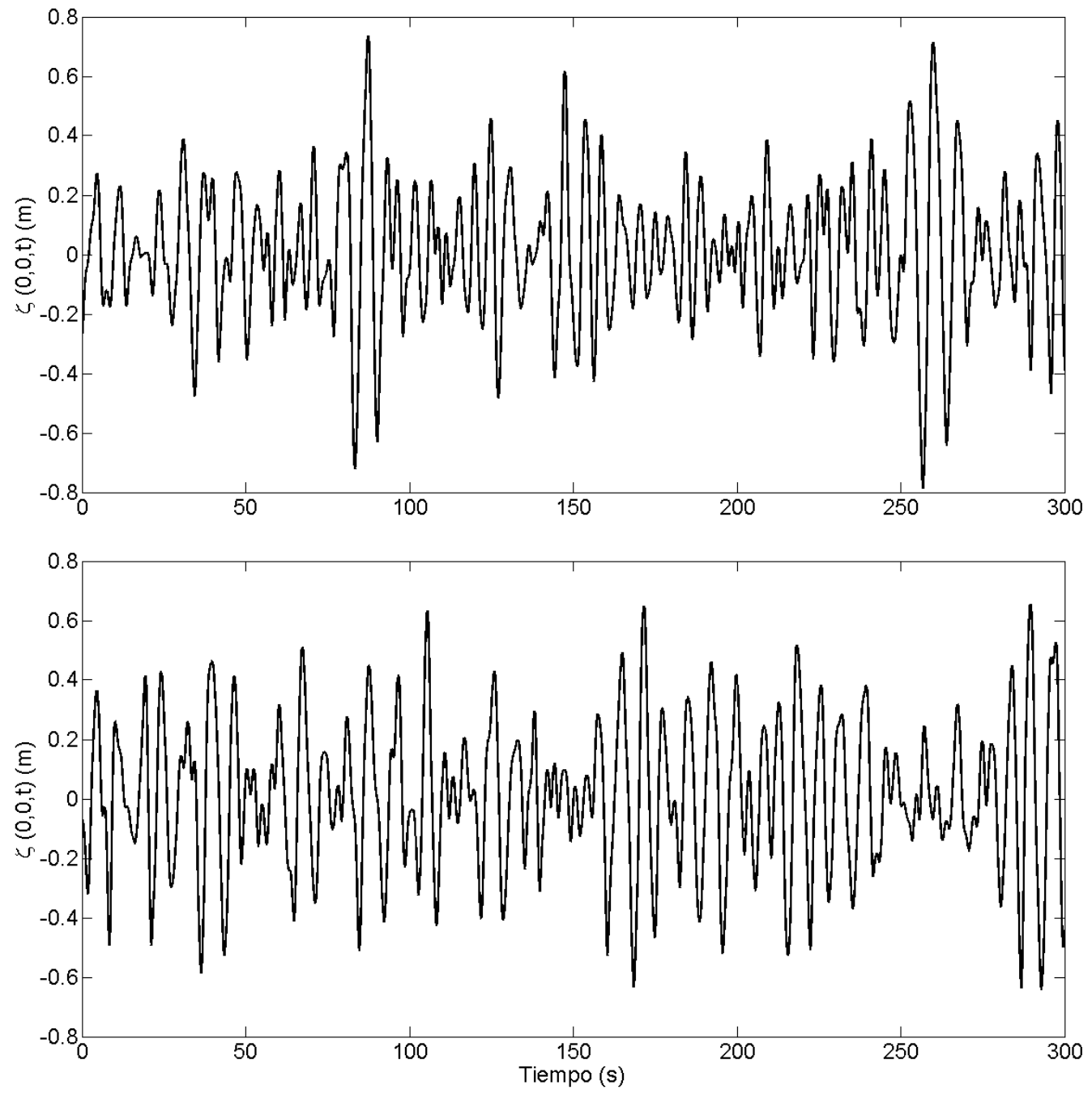

Figura 2.5: Evolución a lo largo del tiempo de la elevación superficial en el origen empleando los modelos long crested (arriba) y short crested (abajo). 


\subsubsection{Esfuerzos sobre el submarino}

Ante la presencia de una ola regular, el submarino experimenta esfuerzos de la forma

$$
Z=-\frac{b}{2}+a \cos \left(\omega_{e} t\right)-\frac{b}{2} \cos \left(2 \omega_{e} t\right)
$$

es decir, existe un esfuerzo oscilante con la frecuencia de excitación, un armónico al doble de esa frecuencia que es habitualmente despreciado, y un esfuerzo de segundo orden que toma un valor constante y que se corresponde con la fuerza de succión. Si bien pueden considerarse otros esfuerzos, lo más usual es considerar que los esfuerzos de primer orden se componen de una fuerza vertical y un momento de giro alrededor del eje $Y$, mientras que la fuerza de succión es una fuerza vertical.

\section{Esfuerzos de primer orden}

Aplicando el principio de superposición, podemos suponer que los esfuerzos totales sobre el vehículo son la suma de los producidos por cada una de las componentes regulares, de modo que para los esfuerzos tendremos de nuevo una suma de $N$ ondas senoidales:

$$
\begin{aligned}
& F_{z 0}(t)=\sum_{i=1}^{N} F_{i} \cos \left(w_{e, i} t-k_{i}(x \cos \chi-y \sin \chi)+\Phi_{F, i}\right) \\
& M_{y}(t)=\sum_{i=1}^{N} M_{i} \cos \left(w_{e, i} t-k_{i}(x \cos \chi-y \sin \chi)+\Phi_{M, i}\right)
\end{aligned}
$$

donde $F_{i}, M_{i}$ son las amplitudes de cada una de las componentes y $\Phi_{F, i}, \Phi_{M, i}$ las fases iniciales.

Existen diversos procedimientos analíticos para la obtención de estos esfuerzos, los más comunes basados en strip theory [STF70], consistente en suponer al vehículo formado por un número finito de paneles transversales bidimensionales rígidamente unidos. En nuestro caso, sin embargo, podemos hacer uso de los resultados experimentales proporcionados por la empresa SSPA [Bys01b]. Estos resultados, obtenidos mediante ensayo con modelo a escala, nos proporcionan valores adimensionales del módulo y la fase de estos esfuerzos. En concreto,

$$
\begin{aligned}
\bar{F}_{z 0} & =\frac{F_{z 0}}{\rho g H L^{2}} \\
\bar{M}_{y} & =\frac{M_{y}}{\rho g H L^{3}},
\end{aligned}
$$

donde $L$ es la longitud del vehículo y $H$ la altura pico-pico de la ola, para ondas regulares con diferentes longitudes de onda y ángulos de encuentro de 0, 45, 90, 135 y 180 grados. En la Figura 2.6 se muestra un ejemplo de los datos obtenidos mediante este procedimiento experimental. Mediante el ajuste por mínimos cuadrados de los datos medidos podemos 
obtener expresiones analíticas para las amplitudes y fases de fuerza y momento en función de la longitud de onda y para ángulos de encuentro de 0, 45, 90, 135 y 180 grados. Un procedimiento similar fue empleado en [Bys88]. En la Figura 2.7 puede verse el resultado de los ajustes correspondientes a las amplitudes de fuerza y momento para un ángulo de 180 grados. El resultado de todos los ajustes así como las expresiones analíticas obtenidas puede encontrarse en el Anexo.
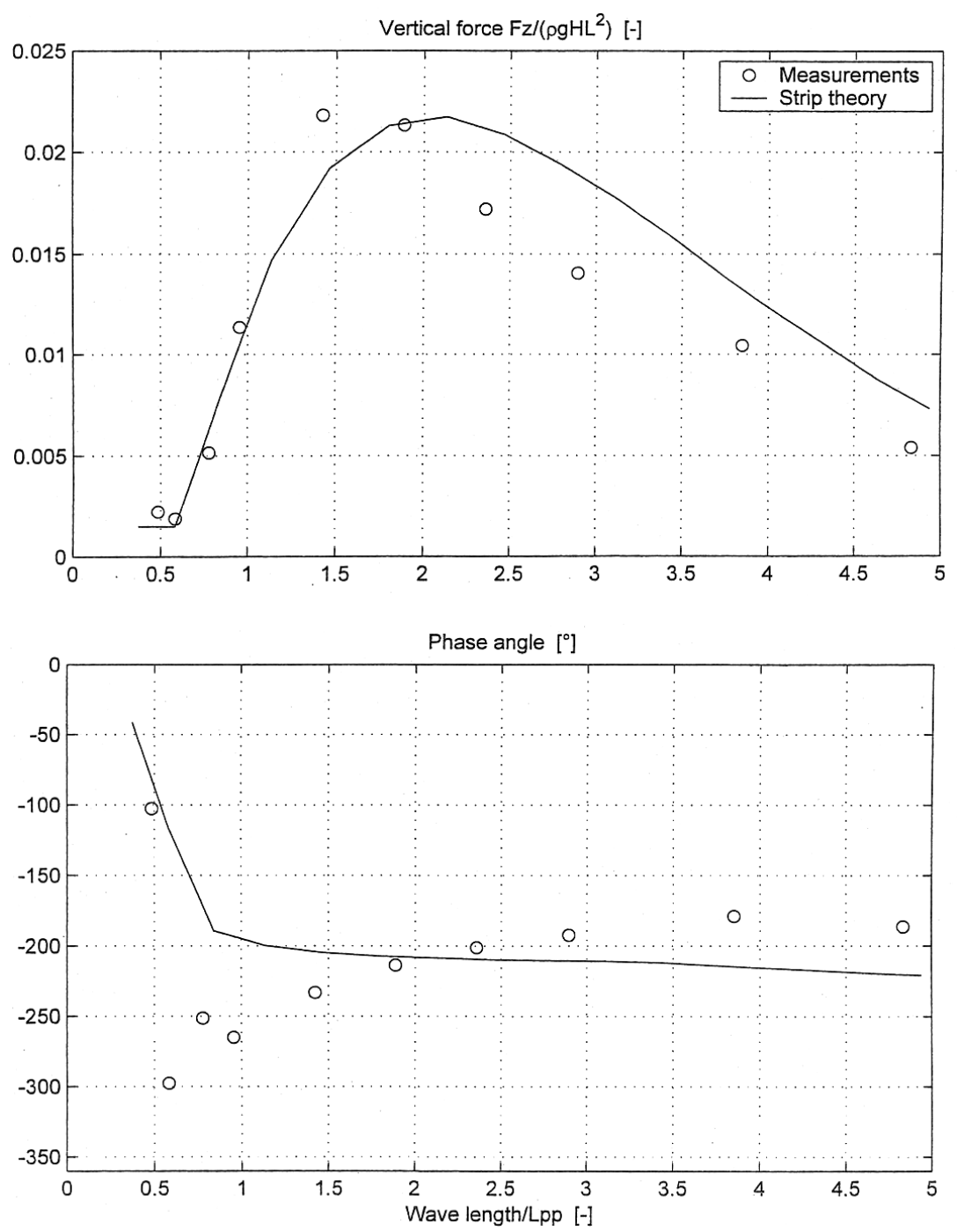

Figura 2.6: Módulo y fase de la fuerza vertical para ángulo de encuentro de $135^{\circ}$. Los valores medidos se muestran como círculos mientras que la línea sólida representa los valores teóricos calculados mediante strip theory. Imagen tomada de [Bys01b]. 

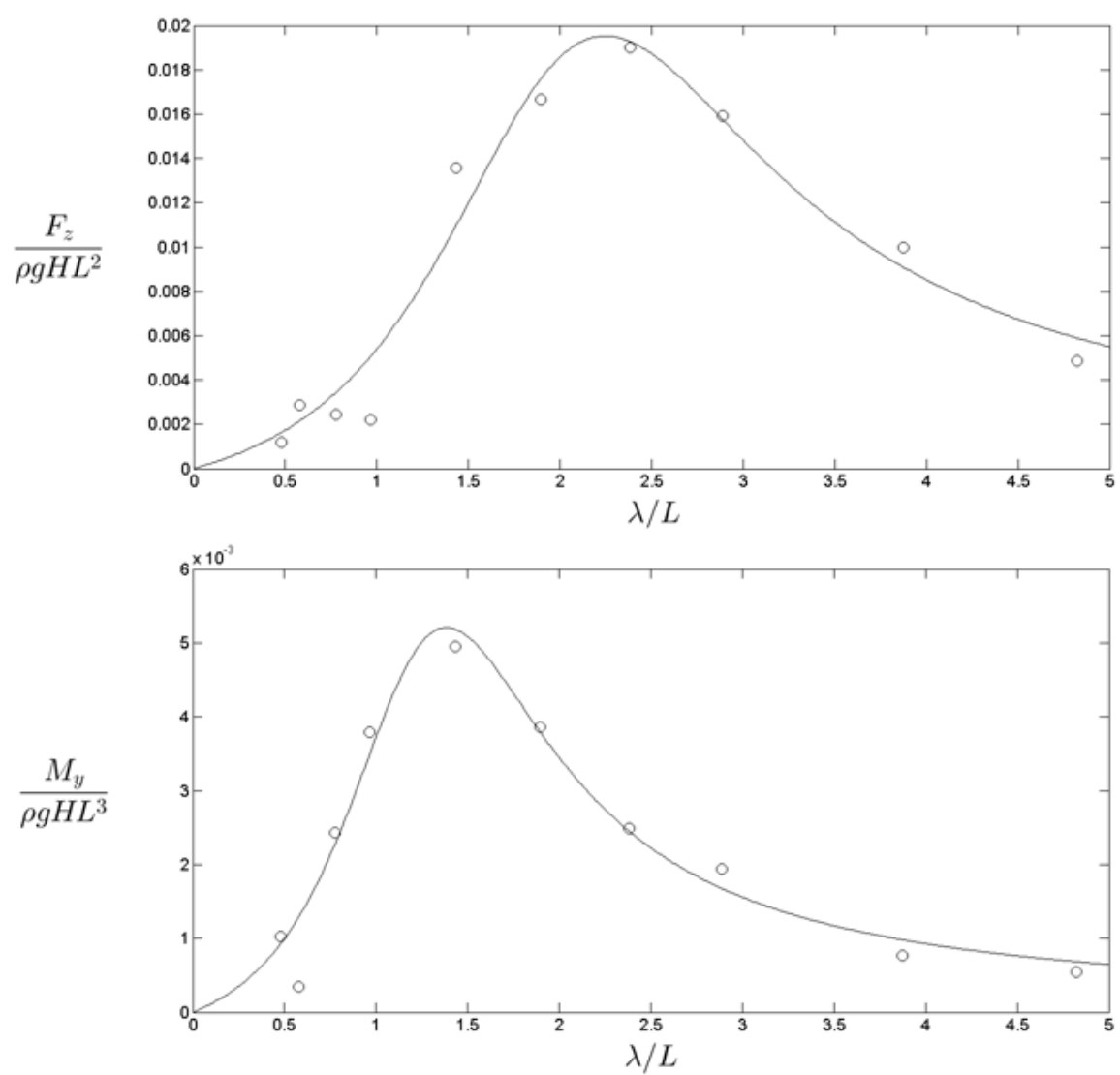

Figura 2.7: Amplitud de la fuerza vertical (arriba) y el momento de giro (abajo) en función de la longitud de onda para ángulo de encuentro de $180^{\circ}$. Los datos medidos se representan mediante círculos mientras que la línea sólida muestra el ajuste obtenido.

Efecto de la profundidad: Los valores dados por las ecuaciones (2.13) y (2.14) son válidos a la profundidad nominal para la que se obtuvieron los datos experimentales, en este caso la profundidad snorkel, $z_{0}=15,5 \mathrm{~m}$ referidos a la quilla para el $\mathrm{P}-650$. A medida que la profundidad aumenta, los esfuerzos se ven atenuados exponencialmente según $\mathrm{e}^{-k\left(z(t)-z_{0}\right)}$. Por tanto, las expresiones finales para $F_{y}$ y $M_{z}$ son:

$$
\begin{aligned}
& F_{z 0}(t)=\sum_{i=1}^{N} \mathrm{e}^{-k_{i}\left(z(t)-z_{0}\right)} F_{i} \cos \left(w_{e, i} t-k_{i}(x \cos \chi-y \sin \chi)+\Phi_{F, i}\right) \\
& M_{y}(t)=\sum_{i=1}^{N} \mathrm{e}^{-k_{i}\left(z(t)-z_{0}\right)} M_{i} \cos \left(w_{e, i} t-k_{i}(x \cos \chi-y \sin \chi)+\Phi_{M, i}\right)
\end{aligned}
$$

En resumen, la metodología propuesta para la obtención de los esfuerzos de primer orden producidos sobre el submarino consiste en: 
1. Considerar una velocidad de avance $U$ y un ángulo $\chi$ respecto al oleaje.

2. Para un estado de mar dado, obtener los valores de $H_{1 / 3}$ y $T_{z}$ de la Tabla 2.2 .

3. Elegir un número de ondas regulares a considerar. $N=100$ suele considerarse suficiente para obtener resultados precisos.

4. Tomar el intervalo de frecuencias de interés y dividirlo en $N$ subintervalos iguales. En el presente trabajo se ha tomado $\omega_{\min }=0,2, \omega_{\max }=3,5$ y $\Delta \omega=\frac{\omega_{\max }-\omega_{\min }}{N}$.

5. Tomar una frecuencia aleatoria, $\omega_{i}$, dentro de cada uno de los subintervalos.

6. Calcular $S\left(\omega_{i}\right) \quad \forall i=1 \ldots N$ mediante (2.5).

7. Calcular la amplitud $A_{i}$ de cada una de las componentes mediante (2.6).

8. Calcular las frecuencias de encuentro $\omega_{e, i}=\omega_{i}-\frac{\omega_{i}^{2}}{g} U \cos \chi$.

9. Tomar, para cada componente, una fase inicial aleatoria en el intervalo $[0,2 \pi)$.

10. Adimensionalizar la longitud de onda de cada una de las componentes con la longitud del vehículo, $\bar{\lambda}_{i}=\frac{\lambda_{i}}{L}$.

11. A partir de $\bar{\lambda}_{i}$ y $\chi$, obtener los valores de amplitudes, $\bar{F}_{i}, \bar{M}_{i}$, y fases, $\Phi_{F, i}, \Phi_{M, i}$, de fuerza y momento mediante los ajustes obtenidos a partir de los datos medidos en canal.

12. Obtener los valores dimensionales de $F_{i}$ y $M_{i}$ a partir de (2.15) y (2.16).

13. Computar la profundidad instantánea $z(t)$.

14. Calcular la fuerza y el momento total mediante las ecuaciones (2.17) y (2.18).

La elevación superficial puede obtenerse mediante (2.11).

La figuras 2.8 y 2.9 muestran la simulación de un estado de mar 5 y los esfuerzos resultantes sobre el submarino para velocidad de avance de 6 nudos y ángulos de encuentro de 180 y 45 grados respectivamente. La comparación de ambas figuras pone de manifiesto el efecto del ángulo relativo sobre la frecuencia de encuentro. Para un ángulo $\chi=180^{\circ}$ (Figura 2.8) el submarino va al encuentro de las olas, resultando en frecuencias pequeñas, mientras que para ángulos cercanos a 0 (Figura $2.9,45^{\circ}$ ), el submarino tiende a alejarse de las olas, por lo que los periodos son mayores. El resultado es que para ángulos cercanos a 180 las rápidas oscilaciones tienden a cancelarse en cierta medida mientras que para periodos mayores los valores permanecen positivos o negativos durante mayor tiempo. Por esta razón, para un estado de mar dado, los mares de popa son más desfavorables que los de proa. Este efecto es más marcado cuanto mayor es la velocidad de avance. 

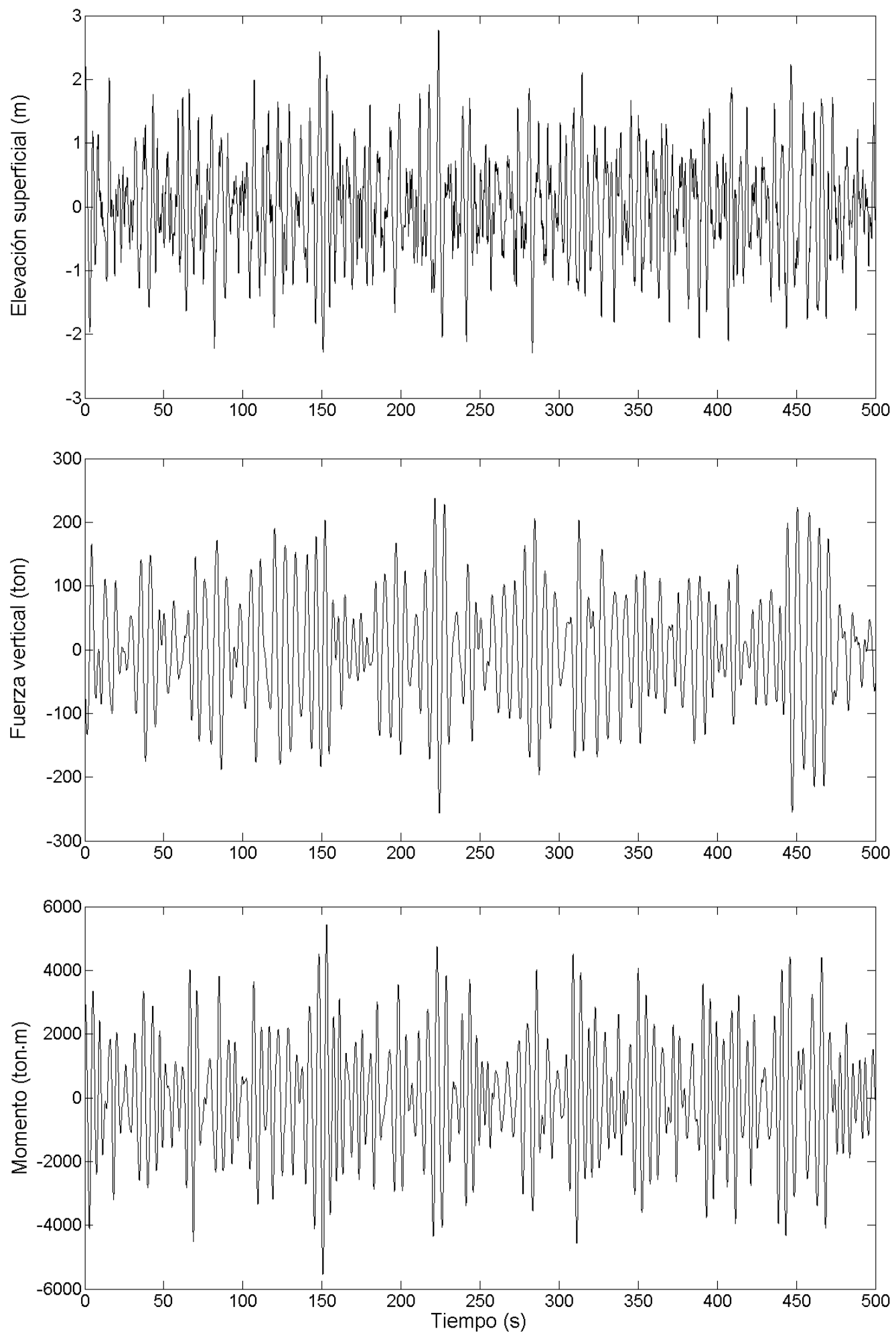

Figura 2.8: Elevación superficial, fuerza vertical y momento de giro para estado de mar 5 con ángulo de encuentro $\chi=180^{\circ}$ y velocidad $U=6 \mathrm{kn}$. 

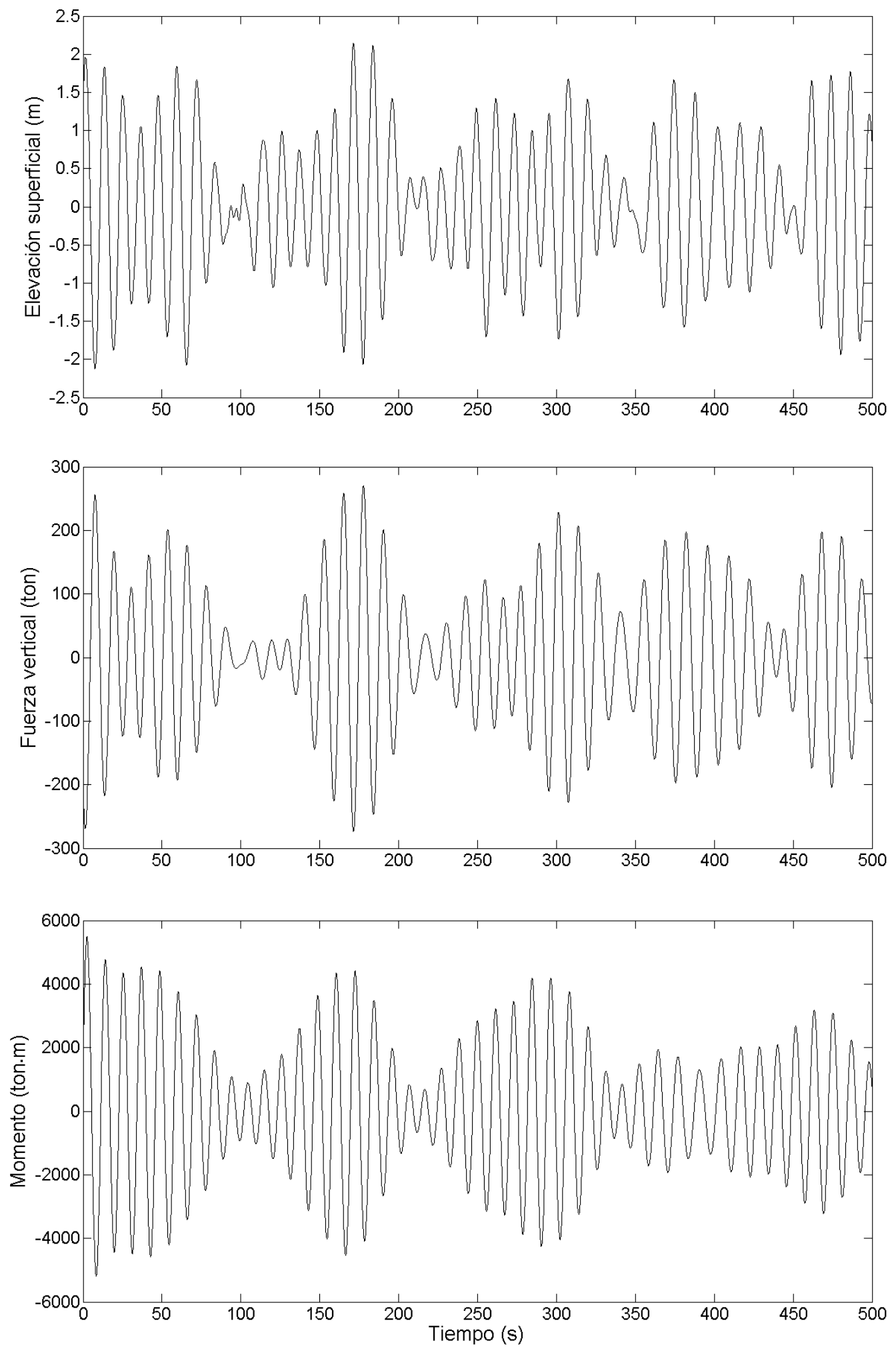

Figura 2.9: Elevación superficial, fuerza vertical y momento de giro para estado de mar 5 con ángulo de encuentro $\chi=45^{\circ}$ y velocidad $U=6 \mathrm{kn}$. 
Nótese que en este experimento se ha simulado un estado de mar y calculado los esfuerzos que el submarino, moviéndose con velocidad constante $U$, experimentaría a cota snorkel nominal, $z_{0}$. Sin embargo, el propio submarino -las ecuaciones del movimiento- no está incluido en la simulación. En ausencia de un sistema de control que estabilizara el vehículo, los grandes esfuerzos (ver figuras 2.8 y 2.9) tenderían a sacar el vehículo a la superficie.

\section{Fuerza de succión}

Además de los esfuerzos de primer orden, existe una fuerza vertical de succión que tiende a atraer el submarino hacia la superficie. Bajo una onda regular, esta fuerza de succión toma un valor constante. Bajo un mar irregular, sin embargo, esta fuerza es también irregular y no sigue una distribución gausiana. Según [Boo83], habitualmente toma valores bajos durante varios minutos para luego experimentar fuertes subidas. En general se considera que es proporcional al cuadrado de la elevación y, al igual que los esfuerzos de primer orden, decae exponencialmente con la profundidad.

Puesto que la fuerza de succión es pequeña en comparación con los esfuerzos de primer orden, la solución adoptada a lo largo de este trabajo ha sido considerarla constante y de magnitud proporcional al valor medio del cuadrado de la elevación. El informe [Bys01b] proporciona el valor medio de la fuerza de succión para estados de mar 5 y 6 . Simulando estos estados de mar durante tiempos lo suficientemente largos puede obtenerse un valor medio del cuadrado de la elevación y por tanto la constante de proporcionalidad entre éste y la fuerza de succión. Repitiendo este experimento para el resto de estados de mar se obtiene el valor promedio de la fuerza de succión que se empleará en las simulaciones:

\section{Estado de mar 6: 4.3 ton}

Estado de mar 5: 1.8 ton

Estado de mar 4: 0.6 ton

Estado de mar 3: 0.13 ton

Como se ha dicho, la fuerza de succión, del mismo modo que los esfuerzos de primer orden, decae exponencialmente con la profundidad. Al igual que éstos lo hará según una ley de la forma $\mathrm{e}^{-k\left(z(t)-z_{0}\right)}$, donde en este caso $k$ se ha tomado correspondiendo al periodo pico del espectro [Bys01b], $T_{\text {peak }}=1,408 \cdot T_{z}$.

Finalmente, es necesario expresar la fuerza total resultante en el sistema de referencia local. Sean $F_{z 0}, F_{\text {suc }}$ las fuerzas correspondientes a los esfuerzos de primer orden y a la fuerza de succión respectivamente, la fuerza total es $F_{T}=F_{z 0}-F_{\text {suc }}$ (la fuerza vertical es positiva hacia abajo). Empleando la transformación de coordenadas (1.1), la fuerza total debida a las 
olas expresada en ejes locales es:

$$
\begin{aligned}
& F_{w, x}=-F_{T} \sin \theta \\
& F_{w, y}=F_{T} \cos \theta \sin \phi \\
& F_{w, z}=F_{T} \cos \theta \cos \phi
\end{aligned}
$$

\subsection{Flotabilidad variable}

En el desarrollo de las ecuaciones del movimiento llevado a cabo en el Capítulo 1 se supuso que la flotabilidad del vehículo tomaba un valor constante $B$. En realidad, el valor de la flotabilidad (peso del volumen de fluido desplazado) se va a ver afectado principalmente por dos fenómenos: en primer lugar la variación en el volumen del vehículo causada por la presión exterior, dependiente de la profundidad, y en segundo lugar las variaciones en la densidad del agua, que modificarán el peso de un determinado volumen de fluido. Si bien la compresibilidad del vehículo puede ser supuesta conocida, puesto que su efecto neto depende en última instancia de la densidad del agua (la variación en la flotabilidad será el producto de la variación de volumen por la variación en la densidad), resulta lógico tratar ambos efectos de forma conjunta como una perturbación externa. Las secciones 2.2.1 y 2.2.2 cubrirán en detalle cada uno de estos fenómenos.

\subsubsection{Efecto de la compresibilidad de los materiales}

La presión hidroestática en el exterior del vehículo es la suma de la presión atmosférica y el peso de la columna de agua sobre él:

$$
p(z)=p_{a t m}+\rho g z
$$

Es decir, la presión exterior crece a medida que aumenta la cota. Esta creciente presión tenderá a comprimir el vehículo.

En estado de equilibrio, para una cierta cota, la flotabilidad del vehículo es igual a su peso. Sin embargo, si el submarino es desplazado de su posición de equilibrio aumentando su cota, la presión exterior comprimirá el casco resistente y otros materiales reduciendo la flotabilidad del vehículo y acelerando su descenso. Del mismo modo, una disminución en la cota tenderá a acelerar el vehículo hacia la superficie. Esta inestabilidad en el plano vertical es un efecto importante que debe ser tenido en cuenta.

La variación de volumen debida a la presión exterior afecta principalmente al casco resistente y a los materiales aislantes. Los siguientes epígrafes cubren el modelado de los efectos de la compresibilidad de ambos materiales. 


\section{Compresibilidad del casco resistente}

La compresión del casco resistente por efecto de la presión exterior puede considerarse lineal con la profundidad. Conocida la contracción producida a una determinada cota, por ejemplo $100 \mathrm{~m}$, puede obtenerse una ley para la variación del volumen en función de la profundidad.

Sea $R_{c}$ el radio del casco resistente, igual a la mitad de la manga, $\delta R_{c, 100}$ la disminución en el radio a $100 \mathrm{~m}$ de profundidad y $S D$ el desplazamiento en superficie del submarino (los valores de éstos y el resto de parámetros introducidos en esta sección pueden encontrarse en la Tabla 2.3), la variación relativa de la sección transversal a $100 \mathrm{~m}$ de profundidad puede obtenerse como:

$$
\left.\Delta A_{r e l}\right|_{100}=\frac{\left.\Delta A\right|_{100}}{A}=\frac{\pi\left(R_{c}^{2}-\left(R_{c}-\delta R_{c, 100}\right)^{2}\right)}{\pi R_{c}^{2}}=\frac{\delta R_{c, 100}\left(2 R_{c}-\delta R_{c, 100}\right)}{R_{c}^{2}} .
$$

Si se supone despreciable la contracción longitudinal, puede asumirse que la variación relativa en volumen, y por tanto en masa, toma este mismo valor. Así:

$$
\left.\Delta S D_{\text {rel }}\right|_{100}=\frac{\left.\Delta S D\right|_{100}}{S D}=\left.\Delta A_{\text {rel }}\right|_{100}
$$

y por tanto

$$
\left.\Delta S D\right|_{100}=\left.S D \cdot \Delta A_{\text {rel }}\right|_{100}
$$

Ésta es la pérdida a 100 m (medidos respecto a la quilla). La pérdida en función de la cota, asumiendo comportamiento lineal y teniendo en cuenta que la profundidad debe referirse al origen del sistema de coordenadas, es

$$
\Delta S D(z)=\left.S D \cdot \Delta A_{r e l}\right|_{100} \cdot \frac{z}{100-R_{c}} .
$$

La pérdida de volumen debida a la contracción del casco resistente es por tanto:

$$
\Delta V_{c c}(z)=\frac{\Delta S D(z)}{\rho}=\left.S D \cdot \Delta A_{r e l}\right|_{100} \cdot \frac{z}{\rho\left(100-R_{c}\right)} .
$$

\section{Compresibilidad de aislantes acústicos}

Los aislantes acústicos están compuestos por un material soporte que contiene grandes cantidades de aire atrapadas en su interior. El material soporte se comporta linealmente con la presión, mientras que el aire atrapado lo hace según la ley de Boyle-Mariotte:

$$
\frac{V_{2}}{V_{1}}=\frac{p_{1}}{p_{2}}
$$


Según los datos proporcionados por el fabricante, este comportamiento es el dominante, por lo que podemos considerar que la pérdida de volumen es proporcional, con constante de proporcionalidad $C_{1}$, a la que se obtendría mediante la ley de Boyle. Por tanto, si consideramos las condiciones a cota 0 y $z \mathrm{~m}$, la pérdida relativa de volumen es

$$
\Delta V_{\text {rel }}(z)=\frac{\Delta V}{V_{0}}=C_{1} \cdot \frac{V_{0}-\frac{p_{0} V_{0}}{p(z)}}{V_{0}}=C_{1} \cdot\left(1-\frac{p_{0}}{p(z)}\right),
$$

siendo $p_{0}$ la presión a profundidad $0, p_{0}=p_{a t m}$, y $p(z)$ la presión a la que se encuentran los aislantes,

$$
p(z)=p_{a t m}+\rho g\left(z-x_{a} \sin \theta+z_{a}\right)
$$

con $x_{a}, z_{a}$ las coordenadas en las que se suponen puntualmente localizados.

La ley proporcionada por (2.25) es válida tanto para la pérdida relativa de volumen como para la pérdida absoluta de volumen o masa, difiriendo únicamente el valor de la constante. Para obtener el valor de ésta necesitamos disponer del valor a una determinada cota. Si, en este caso, disponemos del valor de la pérdida de masa a $350 \mathrm{~m}, \Delta m_{a a, 350}$ :

$$
\Delta m_{a a}(z)=C_{2} \cdot\left(1-\frac{p_{a t m}}{p(z)}\right)
$$

y, particularizando para $z=\left(350-R_{c}\right)$,

$$
C_{2}=\frac{\Delta m_{a a, 350}}{1-\frac{p_{a t m}}{p\left(350-R_{c}\right)}}
$$

luego

$$
\Delta m_{a a}(z)=\frac{\Delta m_{a a, 350}}{1-\frac{p_{a t m}}{p\left(350-R_{c}\right)}} \cdot\left(1-\frac{p_{a t m}}{p(z)}\right) .
$$

Finalmente, la variación en el volumen será:

$$
\Delta V_{a a}(z)=\frac{\Delta m_{a a}(z)}{\rho}=\frac{\Delta m_{a a, 350}}{\rho\left(1-\frac{p_{a t m}}{p\left(350-R_{c}\right)}\right)} \cdot\left(1-\frac{p_{a t m}}{p(z)}\right) .
$$

Se desprecia el cambio en las coordenadas del centro de flotabilidad debido a la compresión de los materiales aislantes.

Para el presente caso, con $\Delta m_{a a, 350}=1130 \mathrm{~kg}$ (ver Tabla 2.3), la Figura 2.10 muestra la evolución de la pérdida de masa con la profundidad. 


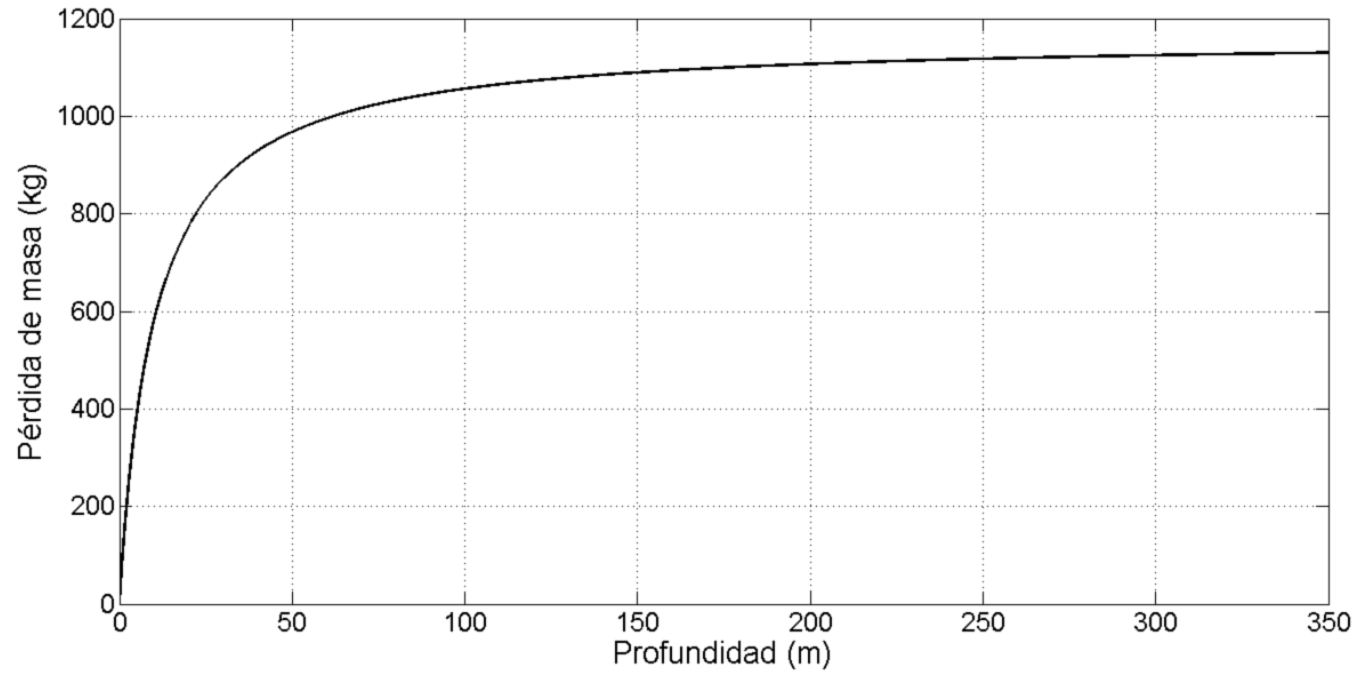

Figura 2.10: Pérdida de flotabilidad debida a la compresión de los aislantes acústicos en función de la cota. 


\subsubsection{Efecto del gradiente de densidad}

La densidad del agua marina varía con la salinidad y la temperatura; esta última, generalmente función de la profundidad. En [FM83] pueden encontrarse los algoritmos para el cálculo de la densidad a partir de estas propiedades.

A efectos de simulación, para el presente trabajo, supondremos que la densidad puede expresarse como una función de la cota. Llamaremos $\rho(z)$ a esta función de la cota mientras que por $\rho$ continuaremos denotando al valor nominal. De este modo, la expresión para la flotabilidad del vehículo como función de la cota, será

$$
B(z)=\rho(z) g\left(\nabla_{f d}-\Delta V_{c c}-\Delta V_{a a}\right)
$$

siendo $\nabla_{f d}$ el volumen correspondiente al desplazamiento de formas, $\nabla_{f d}=\frac{B_{0}}{\rho g}, \mathrm{y} \Delta V_{c c}, \Delta V_{a a}$, las variaciones de volumen debidas respectivamente a la compresión del casco resistente y los materiales aislantes.

La Tabla 2.3 resume el significado y valor de todas las constantes empleadas en el modelo de flotabilidad variable.

\begin{tabular}{|l|c|r|}
\hline Flotabilidad nominal & $B_{0}$ & $23073,1 \mathrm{ton}$ \\
\hline Densidad nominal & $\rho$ & $1,026 \mathrm{ton} / \mathrm{m}^{3}$ \\
\hline Desplazamiento en superficie & $S D$ & $1690 \mathrm{ton}$ \\
\hline Radio del casco resistente & $R_{c}$ & $3,3 \mathrm{~m}$ \\
\hline Contracción del radio a $100 \mathrm{~m}$ & $\delta R_{c, 100}$ & $1 \cdot 10^{-3} \mathrm{~m}$ \\
\hline Pérdida de masa a 350 m por contracción de aislantes & $\Delta m_{a a, 350}$ & $1130 \mathrm{~kg}$ \\
\hline Localización de los aislantes & $x_{a}$ & $3,54 \mathrm{~m}$ \\
& $z_{a}$ & $0,65 \mathrm{~m}$ \\
\hline
\end{tabular}

Tabla 2.3: Valor y significado de los parámetros empleados por el modelo de flotabilidad variable. 



\section{Soplado y ventilación de tanques de lastre}

Los submarinos tripulados están equipados con un número variable de tanques de lastre distribuidos a lo largo de su casco. Cuando estos tanques están llenos de agua proporcionan al vehículo la masa suficiente para sumergirse; en esta condición el peso del vehículo es nominalmente igual a su flotabilidad. En caso de emergencia, como la rotura de uno de los timones o la presencia de una vía de agua, los tanques de lastre pueden ser empleados como un mecanismo de seguridad que permite llevar rápidamente el submarino a la superficie. Para ello, aire a muy alta presión almacenado en unas botellas dispuestas al efecto es inyectado en los tanques forzando la salida del agua al exterior. De esta forma, el submarino se aligera, su flotabilidad aumenta, y tiende a ascender a la superficie. Para llenar los tanques de agua, una válvula situada en su parte superior se abre, de forma que el aire contenido en el tanque escapa al exterior y el agua puede inundar progresivamente el tanque. Estos procesos reciben los nombres, respectivamente, de soplado y ventilación de los tanques de lastre.

El propósito de este capítulo es proponer un modelo matemático para ambos procesos así como para su influencia sobre el comportamiento dinámico del vehículo. Si bien no es una práctica usual, el modelo propuesto contempla la posibilidad de soplar y ventilar de forma simultánea; los modelos matemáticos para ambos procesos considerados de forma individual pueden encontrarse en [FGO10]. 


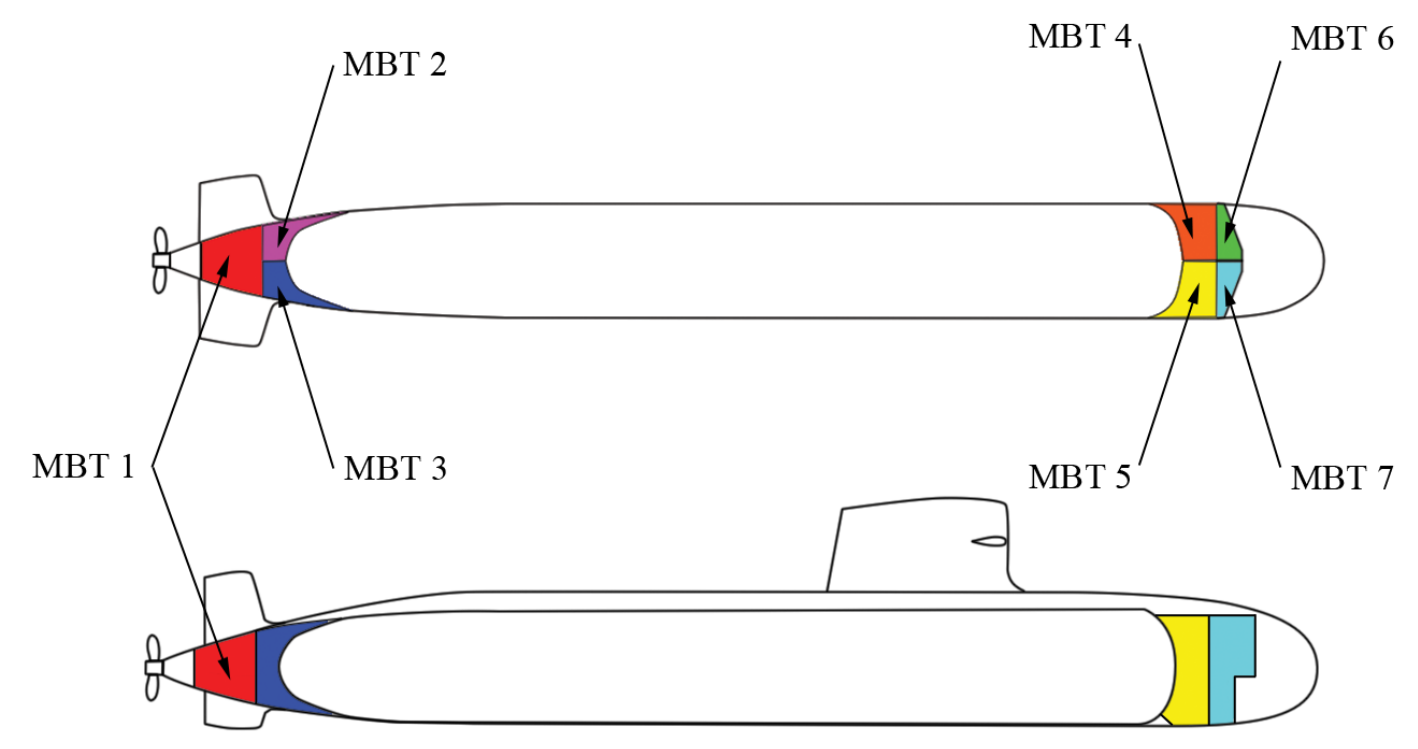

Figura 3.1: Distribución de los tanques de lastre en el submarino P-650. Vista superior (arriba) y lateral (abajo).

\subsection{Tanques de lastre}

El submarino $\mathrm{P}-650$ tiene un total de siete tanques de lastre principales (MBT), con un volumen total de $172,8 \mathrm{~m}^{3}$, lo que supone una capacidad para 177,29 toneladas de agua, aproximadamente un 7,5\% de la masa total del vehículo. La Figura 3.1 muestra la disposición de los tanques así como la numeración adoptada.

Por cada uno de los tanques, el sistema de soplado y ventilación se compone del propio tanque, una botella de aire a alta presión, las válvulas de soplado y ventilación y un orificio situado en la base del tanque que mantiene a éste en contacto con el exterior a través de una rejilla. Cuando la válvula de soplado es abierta, el aire procedente de la botella penetra en el tanque a alta velocidad aumentando la presión y forzando la salida del agua a través del orificio de salida. Cuando la válvula de ventilación es abierta, el aire puede fluir al exterior permitiendo que el agua inunde el tanque. La Figura 3.2 muestra un esquema con las principales variables involucradas en los procesos de soplado y ventilación. El subíndice $F$ denota las condiciones en la botella, el subíndice $B$ las condiciones en el tanque, $\dot{m}_{F}$ y $\dot{m}_{v}$ son respectivamente los gastos másicos a través de los conductos de ventilación y soplado y $q_{B}$ es el caudal a través del orificio situado en la base del tanque. Utilizaremos 3 variables por cada uno de los tanques para describir su estado de forma completa: masa de aire en la botella, $m_{F}$, masa de aire en el tanque, $m_{B}$, y presión en el tanque, $p_{B}$. 
La Tabla 3.1 resume la notación que se introducirá a lo largo de este capítulo.

\begin{tabular}{|l|l|}
\hline$A$ & Área en la garganta de la tobera $\left(\mathrm{m}^{2}\right)$ \\
\hline$A_{h}$ & Área del orificio de salida $\left(\mathrm{m}^{2}\right)$ \\
\hline$A_{v}$ & Área del conducto de ventilación $\left(\mathrm{m}^{2}\right)$ \\
\hline$C_{h}$ & Coeficiente del orificio de salida \\
\hline$H_{t k}$ & Altura del tanque de lastre $(\mathrm{m})$ \\
\hline$h_{w c}(t)$ & Altura de la columna de agua en el tanque $(\mathrm{m})$ \\
\hline$m_{B}(t)$ & Masa de aire en el tanque $(\mathrm{kg})$ \\
\hline$m_{F}(t)$ & Masa de aire en la botella $(\mathrm{kg})$ \\
\hline$\dot{m}_{F}(t)$ & Gasto másico desde la botella $(\mathrm{kg} / \mathrm{s})$ \\
\hline$\dot{m}_{v}(t)$ & Gasto másico a través del conducto de ventilación $(\mathrm{kg} / \mathrm{s})$ \\
\hline$p_{B}(t)$ & Presión en el tanque de lastre $(\mathrm{Pa})$ \\
\hline$p_{e x t}(t)$ & Presión en el exterior del conducto de ventilación $(\mathrm{Pa})$ \\
\hline$p_{F}(t)$ & Presión en la botella $(P a)$ \\
\hline$p_{S E A}(t)$ & Presión en el exterior del orificio de salida $(\mathrm{Pa})$ \\
\hline$q_{B}(t)$ & Caudal de agua a través del orificio del tanque $\left(\mathrm{m}^{3} / \mathrm{s}\right)$ \\
\hline$T_{B}$ & Temperatura del agua $(\mathrm{K})$ \\
\hline$T_{F}(t)$ & Temperatura en la botella $(\mathrm{K})$ \\
\hline$V_{B 0}$ & Volumen inicial de aire en el tanque $\left(\mathrm{m}^{3}\right)$ \\
\hline$V_{B}(t)$ & Volumen de aire en el tanque $\left(\mathrm{m}^{3}\right)$ \\
\hline$V_{B B}$ & Volumen del tanque de lastre $\left(\mathrm{m}^{3}\right)$ \\
\hline$V_{F}$ & Volumen de la botella de aire $\left(\mathrm{m}^{3}\right)$ \\
\hline$\left(x_{b}, y_{b}, z_{b}\right)$ & Coordenadas del centro geométrico del tanque $(\mathrm{m})$ \\
\hline$z_{h}$ & Localización del orificio respecto al origen $(\mathrm{m})$ \\
\hline$z_{t}$ & Localización de la parte superior del tanque respecto al origen $(\mathrm{m})$ \\
\hline$z_{v}$ & Localización de la válvula de ventilación respecto al origen $(\mathrm{m})$ \\
\hline$\gamma$ & Constante isentrópica del aire \\
\hline$\rho$ & Densidad del agua $\left(\mathrm{kg} / \mathrm{m}^{3}\right)$ \\
\hline
\end{tabular}

Tabla 3.1: Variables y símbolos.

El modelo puede dividirse en cuatro partes principales:

- Flujo de aire desde la botella.

- Flujo de aire a través del conducto de ventilación.

- Flujo de agua a través del orificio.

- Evolución de la presión en el tanque. 


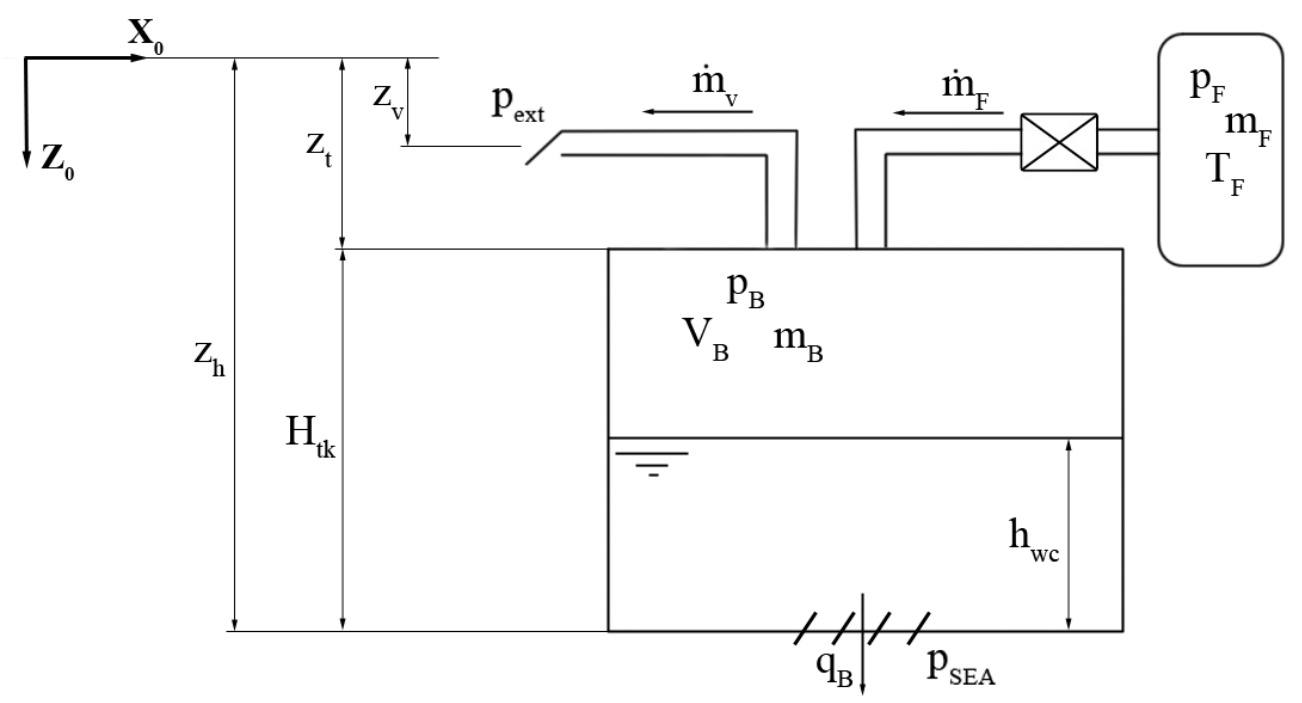

Figura 3.2: Vista esquemática de los procesos de soplado y ventilación.

\subsection{Flujo de aire desde la botella}

Cuando la válvula de soplado se abre, el aire de la botella es inyectado en el tanque a través de una tobera. Por el momento despreciaremos las pérdidas de presión y la transferencia de calor a lo largo del sistema de tuberías que conecta la botella y el tanque. Como veremos más adelante, sin embargo, las pérdidas de presión pueden ser tenidas en cuenta de forma indirecta. En estas condiciones, el problema se reduce a estudiar el flujo unidimensional estacionario de un fluido ideal compresible. El análisis de este problema puede encontrarse en cualquier texto clásico de mecánica de fluidos, si bien se incluye a continuación con el fin de hacer el desarrollo tan autocontenido como sea posible.

Al inicio del proceso de soplado, debido a la gran diferencia de presión entre botella y tanque, el flujo de aire será supersónico; a medida que el aire fluye desde la botella, esta diferencia decrece pasando el flujo a ser subsónico a partir de un determinado momento. Esta transición se producirá cuando la relación entre la presión en la botella, $p_{F}$, y en el tanque, $p_{B}$, sea igual a la relación de presiones crítica,

$$
\left(\frac{p_{F}}{p_{B}}\right)=P_{c}=\left(\frac{\gamma+1}{2}\right)^{\frac{\gamma}{\gamma-1}}
$$

siendo $\gamma$ la constante isentrópica del aire. 
Flujo supersónico: En este caso, resulta sencillo calcular el caudal de aire que atraviesa la garganta de la tobera, donde el número de Mach es igual a la unidad. En concreto,

$$
\dot{m}_{F}=\rho_{a}^{*} v^{*} A=\rho_{a, 0} a_{0} \frac{\rho_{a}^{*}}{\rho_{a, 0}} \frac{a^{*}}{a_{0}} A,
$$

donde $A$ es el área en la garganta de la tobera, $a$ es la velocidad del sonido, $v$ es la velocidad del aire, $\rho_{a}$ es la densidad del aire, el asterisco denota condiciones críticas, donde el número de Mach es la unidad, y el subíndice 0 denota condiciones de remanso. Estas dos condiciones están relacionadas por

$$
\left(\frac{p_{0}}{p^{*}}\right)^{\frac{\gamma-1}{\gamma}}=\left(\frac{\rho_{a, 0}}{\rho_{a}^{*}}\right)^{\gamma-1}=\left(\frac{a_{0}}{a^{*}}\right)^{2}=\frac{T_{0}}{T^{*}}=\frac{\gamma+1}{2},
$$

donde $T$ es la temperatura.

Asumiendo que en este caso las condiciones de remanso corresponden a las condiciones en la botella, haciendo uso de las relaciones anteriores, la ley de los gases ideales $\rho_{a, 0}=\frac{p_{0}}{R_{g} T_{0}}$ y $a_{0}=\sqrt{\gamma R_{g} T_{0}}$, el caudal de aire es

$$
\dot{m}_{F}(t)=\frac{A p_{F}(t)}{\sqrt{R_{g} T_{F}(t)}} \sqrt{\gamma\left(\frac{2}{\gamma+1}\right)^{\frac{\gamma+1}{\gamma-1}}}
$$

donde $R_{g}$ es la constante de los gases para el aire y $T_{F}$ es la temperatura en la botella.

Flujo subsónico: En este caso el caudal de aire viene dado por

$$
\dot{m}_{F}=\rho_{a, e} M_{e} a_{e} A_{e}=\rho_{a, 0} a_{0} \frac{\rho_{a, e}}{\rho_{a, 0}} \frac{a_{e}}{a_{0}} M_{e} A_{e},
$$

donde $A_{e}$ es el área de la sección de salida, en este caso $A_{e}=A, M$ es el número de Mach y el subíndice $e$ denota condiciones en la salida. Las condiciones de salida se relacionan con las de remanso mediante

$$
\frac{T_{0}}{T}=\left(\frac{\rho_{a, 0}}{\rho_{a}}\right)^{\gamma-1}=\left(\frac{p_{0}}{p}\right)^{\frac{\gamma-1}{\gamma}}=1+\frac{\gamma-1}{2} M^{2} .
$$

Particularizando para $p_{0}=p_{F}$ y $p=p_{e}=p_{B}$, el número de Mach en la salida será

$$
M_{e}=\sqrt{\frac{2}{\gamma-1}\left[\left(\frac{p_{F}}{p_{B}}\right)^{\frac{\gamma-1}{\gamma}}-1\right]}
$$

Sustituyendo (3.4) en (3.3) y procediendo como en el caso supersónico, obtenemos

$$
\dot{m}_{F}(t)=\frac{A p_{F}(t)}{\sqrt{R_{g} T_{F}(t)}} \sqrt{\frac{2 \gamma}{\gamma-1}\left[\left(\frac{p_{B}(t)}{p_{F}(t)}\right)^{\frac{2}{\gamma}}-\left(\frac{p_{B}(t)}{p_{F}(t)}\right)^{\frac{\gamma+1}{\gamma}}\right]} .
$$


El gasto másico de aire procedente de la botella puede por tanto expresarse para los casos subsónico y supersónico como

$$
\dot{m}_{F}(t)=\frac{A p_{F}(t)}{\sqrt{R_{g} T_{F}(t)}} \cdot \mu\left(p_{B}(t), p_{F}(t)\right)
$$

$\operatorname{con} \mu\left(p_{B}(t), p_{F}(t)\right)=\mu_{\max }=\operatorname{cte} \operatorname{si} \frac{p_{F}(t)}{p_{B}(t)} \geq\left(\frac{\gamma+1}{2}\right)^{\frac{\gamma}{\gamma-1}}$.

Conforme la presión en la botella disminuye, su temperatura se incrementa. Este proceso puede considerarse adiabático, es decir, puede despreciarse la transmisión de calor. Como se menciona en [Bys04], los resultados experimentales disponibles avalan esta hipótesis. Sean $m_{F 0}, p_{F 0}$ la masa de aire y presión iniciales en la botella y $V_{F}$ el volumen de la botella, bajo las hipótesis anteriores la presión y temperatura instantáneas vendrán dadas por

$$
p_{F}(t)=\left(\frac{m_{F}(t)}{m_{F 0}}\right)^{\gamma} p_{F 0}, \quad T_{F}(t)=\frac{p_{F}(t) V_{F}}{R_{g} m_{F}(t)} .
$$

Por tanto,

$$
\frac{p_{F}(t)}{\sqrt{R_{g} T_{F}(t)}}=\sqrt{\frac{m_{F}(t)^{\gamma+1} p_{F 0}}{m_{F 0}^{\gamma} V_{F}}} .
$$

De esta forma, la expresión final para el gasto másico es

$$
\dot{m}_{F}(t)=A\left(\frac{m_{F}(t)^{\gamma+1} p_{F 0}}{m_{F 0}^{\gamma} V_{F}}\right)^{\frac{1}{2}} \mu\left(p_{B}(t), m_{F}(t)\right)
$$

con

$$
\mu\left(p_{B}, m_{F}\right)=\left\{\begin{array}{cl}
\sqrt{\gamma\left(\frac{2}{\gamma+1}\right)^{\frac{\gamma+1}{\gamma-1}}}, & P_{c} \leq \frac{p_{F}}{p_{B}} \\
\sqrt{\frac{2 \gamma}{\gamma-1}\left(\frac{p_{B}}{\left.\left.p_{F 0}\left(\frac{m_{F}}{m_{F 0}}\right)^{\gamma}\right)^{\frac{2}{\gamma}}-\left(\frac{p_{B}}{p_{F 0}\left(\frac{m_{F}}{m_{F 0}}\right)^{\gamma}}\right)^{\frac{\gamma+1}{\gamma}}\right)},\right.} & 1<\frac{p_{F}}{p_{B}}<P_{c} \\
0, & \frac{p_{F}}{p_{B}} \leq 1
\end{array}\right.
$$

Puesto que el caudal inicial depende exclusivamente de las condiciones iniciales en la botella, puede considerarse a éste como una constante de valor

$$
\dot{m}_{F}(0)=A\left(\gamma\left(\frac{2}{\gamma+1}\right)^{\frac{\gamma+1}{\gamma-1}} \frac{p_{F 0} m_{F 0}}{V_{F}}\right)^{\frac{1}{2}} .
$$

Este caudal inicial ha sido medido para diferentes intensidades de soplado. Sea $\dot{m}_{F \max }$ el valor medido del caudal máximo. En lugar de emplear el valor numérico del área $A$ al calcular (3.8), 
lo obtendremos de (3.10) y el valor medido de $\dot{m}_{F \max }$, es decir,

$$
A=\dot{m}_{F \max }\left(\left(\frac{2}{\gamma+1}\right)^{-\frac{\gamma+1}{\gamma-1}} \frac{V_{F}}{\gamma p_{F 0} m_{F 0}}\right)^{\frac{1}{2}} .
$$

Haciendo esto nos aseguramos de que el caudal inicial calculado coincide con el valor real medido. De este modo, aunque las pérdidas de presión no son consideradas explicitamente en el modelo, son tenidas en cuenta de forma indirecta.

\subsection{Flujo de aire a través del conducto de ventilación}

Las ecuaciones para el flujo de aire desde el tanque pueden obtenerse de forma análoga a la empleada para el flujo de aire desde la botella. En este caso las presiones a evaluar son la presión en el tanque, $p_{B}$, y la presión en el exterior del conducto de ventilación, $p_{\text {ext }}=p_{a t m}+\rho g\left(z+z_{v}-x_{b} \sin \theta\right)$, donde $p_{a t m}$ es la presión atmosférica, $z$ es la cota, $z_{v}$ es la distancia vertical entre el origen del sistema de coordenadas cuerpo y la válvula de ventilación, y el término $x_{b} \sin \theta$ representa la variación en la cota a la que se encuentra el tanque con el ángulo de pitch. Así, $\left(z+z_{v}-x_{b} \sin \theta\right)$ es la profundidad a la que se encuentra la salida del sistema de ventilación. Por lo tanto, de forma similar a (3.6), tenemos

$$
\dot{m}_{v}(t)=\bar{\mu}\left(p_{B}, p_{e x t}\right) \frac{A_{v} p_{B}(t)}{R_{g} T_{B}(t)}
$$

donde $A_{v}$ es la sección del conducto de ventilación y

$$
\bar{\mu}=\bar{\mu}_{\max } \quad \text { si } \quad \frac{p_{\text {ext }}}{p_{B}} \leq\left(\frac{p_{\text {ext }}}{p_{B}}\right)_{\text {crit }} .
$$

Si bien los detalles geométricos del sistema de ventilación no están disponibles y por tanto las pérdidas de presión no pueden ser directamente calculadas, sí contamos con una estimación de $\bar{\mu}\left(p_{B}, p_{\text {ext }}\right)$ para distintos valores de $\frac{p_{\text {ext }}}{p_{B}}$ teniendo en cuenta las distintas pérdidas de presión presentes en el sistema. En concreto, la relación de presiones crítica se estima en $\left(\frac{p_{e x t}}{p_{B}}\right)_{\text {crit }}=0,5184$ y $\bar{\mu}_{\text {max }}=0,3550$. Sea $\Pi=\frac{p_{e x t}}{p_{B}}$ la relación de presiones, el ajuste por mínimos cuadrados de estos datos (ver Figura 3.3) proporciona la siguiente expresión para $\bar{\mu}$ :

$$
\bar{\mu}(\Pi)=\left\{\begin{array}{cll}
\bar{\mu}_{\max } & \text { si } \quad \Pi \leq \Pi_{\text {crit }} \\
\frac{-66,97 \Pi^{2}-52,70 \Pi+119,70}{\Pi^{3}-171,30 \Pi^{2}-75,65 \Pi+294,60} & \text { si } & \Pi_{\text {crit }}<\Pi<1 \\
0 & \text { si } \quad \Pi \geq 1 .
\end{array}\right.
$$




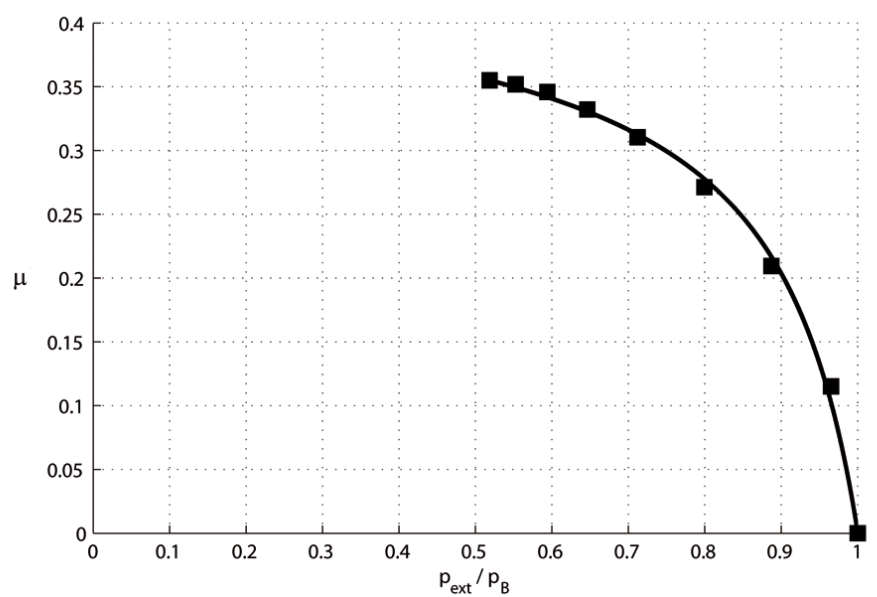

Figura 3.3: Resultado del ajuste por mínimos cuadrados de $\bar{\mu}(\Pi)$ teniendo en cuenta las pérdidas de presión.

La variación en la masa de aire en el tanque es la diferencia entre el caudal procedente de la botella y el que sale a través de la válvula de ventilación. De este modo, usando (3.12) y (3.13),

$$
\dot{m}_{B}(t)=-\dot{m}_{F}(t)-\bar{\mu}(\Pi(t)) \frac{A_{v} p_{B}(t)}{R_{g} T_{B}(t)} .
$$

\subsection{Flujo de agua a través del orificio}

La diferencia de presiones entre el tanque y el océano forzará al agua a fluir hacia o desde el tanque a través del orificio situado en su base. Supongamos, en primer lugar, que la presión en el tanque es superior a la presión exterior; en este caso el agua abandonará el tanque. La presión inmediatamente sobre el orificio de salida es $p_{B}(t)+\rho g h_{w c}(t)$, donde $h_{w c}(t)$ es la altura de la columna de agua en el tanque. Suponiendo que el tanque tiene una forma regular, esta altura puede expresarse como

$$
h_{w c}(t)=\left(1-\frac{\Delta m(t)}{\Delta m_{\max }}\right) H_{t k}
$$

donde $H_{t k}$ es la altura del tanque, $\Delta m(t)$ es la variación en la masa de agua en el tanque y $\Delta m_{\max }$ es el valor máximo de esta variación (véase la Sección 3.7 para más detalles sobre estas dos últimas variables).

La ecuación de Bernoulli aplicada a ambos lados del orificio de salida proporciona

$$
p_{B}(t)+\rho g h_{w c}(t)-\Delta p_{\text {loss }}=p_{S E A}(t)+\frac{1}{2} \rho v_{h}^{2}(t),
$$


donde $v_{h}$ y $\Delta p_{\text {loss }}$ son, respectivamente, la velocidad y pérdida de presión en el orificio de salida. Las pérdidas de presión debidas tanto a la fricción como al cambio de sección fueron calculadas mediante un modelo independiente y pueden ser agrupadas en el término

$$
\Delta p_{\text {loss }}=\zeta_{h} \frac{1}{2} \rho v_{h}^{2}(t)
$$

$\zeta_{h}$ fue estimada en $\zeta_{h}=2,5$.

De esta forma la velocidad en el orificio de salida es

$$
v_{h}(t)=\sqrt{\frac{2\left(p_{B}(t)+\rho g h_{w c}(t)-p_{S E A}(t)\right)}{\rho\left(1+\zeta_{h}\right)}},
$$

y el caudal de agua que abandona el tanque

$$
q_{B}(t)=C_{h} A_{h} v_{h}(t)=C_{n} A_{h} \sqrt{\frac{2\left(p_{B}(t)+\rho g h_{w c}(t)-p_{S E A}(t)\right)}{\rho\left(1+\zeta_{h}\right)}},
$$

donde

$$
p_{S E A}=p_{a t m}+\rho g\left(z+z_{h}-x_{b} \sin \theta\right) .
$$

Aquí $z_{h}$ es la localización del orificio de salida, $A_{h}$ su área y $C_{h}$ un coeficiente que tiene en cuenta que, puesto que este orificio está dotado de una rejilla, el área efectiva es menor que $A_{h}$.

Si la presión en el tanque es menor que la exterior, entonces el agua fluirá hacia el tanque. En este caso la expresión para el caudal vendrá dada por (3.16) con signo negativo.

\subsection{Evolución de la presión en el tanque}

Cuando se inicia el soplado el aire penetra en el tanque a gran velocidad mezclándose con el agua rápidamente, lo que favorece una rápida transferencia de calor entre el aire y el agua contenida en el tanque. Este hecho nos permite asumir que el aire adoptará inmediatamente la temperatura en el tanque. De este modo, el proceso puede considerarse isotermo. Según [Bys04], resultados experimentales avalan esta suposición.

Derivando respecto al tiempo la ley de los gases perfectos obtenemos

$$
\dot{p}_{B}(t)=\frac{\dot{m}_{B}(t) R_{g} T_{B}-\dot{V}_{B}(t) p_{B}(t)}{V_{B}(t)} .
$$

El volumen ocupado en el tanque por el aire aumenta en la misma medida que el ocupado por el agua disminuye; por tanto, la velocidad a la que éste cambia, $\dot{V}_{B}$, será el caudal de agua $q_{B}$ dado por (3.16). Haciendo uso de la ecuación de los gases perfectos, el volumen de 
aire en el tanque en un cierto instante de tiempo será $V_{B}=\frac{m_{B} R_{g} T_{B}}{p_{B}}$. La variación de la presión en el tanque será por tanto:

$$
\dot{p}_{B}(t)-\frac{p_{B}(t)}{m_{B}(t)} \dot{m}_{B}(t)=-\frac{p_{B}^{2}(t) q_{B}(t)}{m_{B}(t) R_{g} T_{B}} .
$$

Desde los puntos de vista matemático y numérico, la presencia de una raíz cuadrada en la ecuación (3.16) supone una seria dificultad dado que si el término dentro de la raíz se aproxima a cero, el gradiente tenderá a infinito. Para evitar este problema, aproximaremos la raíz cuadrada alrededor del origen $(x \in[0, \xi])$ por un polinomio de cuarto grado:

$$
P(x)=a_{0}+a_{1} x+a_{2} x^{2}+a_{3} x^{3}+a_{4} x^{4} .
$$

Los valores de los coeficientes deben ser tales que se cumplan las siguientes condiciones:

$$
\begin{cases}\text { (a) } & P(0)=0 \\ \text { (b) } & P(\xi)=\sqrt{\xi} \\ \text { (c) } & \dot{P}(0)=0 \\ \text { (d) } & \dot{P}(\xi)=\frac{1}{\sqrt{\xi}} \\ \text { (e) } & \int_{0}^{\xi} P(x) d x=\int_{0}^{\xi} \sqrt{x} d x .\end{cases}
$$

Nótese que las condiciones (a)-(d) exigen la continuidad de $P$ y su derivada primera, $\dot{P}$, en los extremos $x=0, \xi$, y la condición (e) supone la conservación del valor medio del caudal.

Ensayos numéricos mostraron que $\xi=1$ proporciona una buena aproximación para la raíz cuadrada en (3.16). De esta forma, obtenemos

$$
a_{0}=a_{1}=0, \quad a_{2}=8,75, \quad a_{3}=-14 \quad \text { and } \quad a_{4}=6,25 .
$$

Resumiendo, consideraremos la nueva función

$$
\bar{P}(x)=\left\{\begin{array}{lll}
-\sqrt{-x} & \text { si } & x<-\xi \\
-P(-x) & \text { si } & -\xi \leq x \leq 0 \\
P(x) & \text { si } & 0<x \leq \xi \\
\sqrt{x} & \text { si } & x>\xi
\end{array}\right.
$$

y sustituiremos $q_{B}(t)$ por su aproximación $\bar{q}_{B}(t)$ definida como

$$
\bar{q}_{B}(t)=C_{n} A_{h} \bar{P}\left(\frac{2\left(p_{B}(t)+\rho g h_{w c}(t)-p_{S E A}(t)\right)}{\rho\left(1+\zeta_{h}\right)}\right) .
$$

\subsection{Control del sistema de soplado--ventilación}

Las expresiones para $\dot{m}_{F}$ y $\dot{m}_{v}$ proporcionadas por las ecuaciones (3.6) y (3.12) son válidas en caso de que las válvulas de soplado y ventilación respectivamente estén completamente 
abiertas. Con el fin de controlar ambos procesos, introduciremos dos nuevos conjuntos de variables que modelarán el grado de apertura de las válvulas de soplado y ventilación. Llamaremos $s_{i} \in L^{\infty}\left(0, t_{f} ;[0,1]\right)$ a la apertura de la válvula de soplado del $i$-ésimo tanque durante el intervalo $\left[0, t_{f}\right]$. El valor 0 indica que la correspondiente válvula se encuentra completamente cerrada mientras que el valor 1 se corresponde con la total apertura de la misma. Todos los valores intermedios son admisibles. Del mismo modo, denotaremos por $\bar{s}_{i} \in L^{\infty}\left(0, t_{f} ;[0,1]\right)$ a la apertura de la válvula de ventilación del $i$-ésimo tanque.

De este modo, a partir de (3.8), (3.14) y (3.17), obtenemos la forma final de las ecuaciones que gobiernan la evolución de la masa de aire en la botella $\left(m_{F_{i}}(t)\right)$, la masa de aire en el tanque $\left(m_{B_{i}}(t)\right)$, y la presión en el tanque $\left(p_{B_{i}}(t)\right)$ bajo la acción de las variables de control $s_{i}, \bar{s}_{i}$. Obtenemos,

$$
\begin{gathered}
\dot{m}_{F i}(t)=s_{i}(t) A\left(\frac{m_{F i}(t)^{\gamma+1} p_{F 0}}{m_{F 0}^{\gamma} V_{F}}\right)^{\frac{1}{2}} \mu_{i}\left(p_{B i}(t), m_{F i}(t)\right) \\
\dot{m}_{B i}(t)+\dot{m}_{F i}(t)=-\bar{\mu}_{i}(\Pi(t)) \frac{\bar{s}_{i}(t) A_{v} p_{B i}(t)}{\sqrt{R_{g} T_{B}}}, \\
\frac{m_{B i}(t)}{p_{B i}(t)} \dot{p}_{B i}(t)-\dot{m}_{B i}(t)=-\frac{p_{B i}(t) \bar{q}_{B i}(t)}{R_{g} T_{B}} .
\end{gathered}
$$

Esta formulación asume que el flujo a través de las válvulas varía linealmente con su apertura. Por supuesto, en caso de que este sistema de control fuera incorporado en un vehículo real, esta suposición debería ser adaptada a las características particulares de las válvulas instaladas.

A lo largo de este trabajo se considerará el soplado/ventilación de los tanques del 2 al 5 (ver Figura 3.1). Los valores de todos los parámetros geométricos necesarios pueden encontrarse en la Tabla 3.2 .

\subsection{Masa variable}

La entrada o salida de agua de los tanques producirá variaciones de masa localizadas en distintos puntos del vehículo. Puesto que las ecuaciones del movimiento consideran constante la masa del vehículo, es necesario modificarlas para incorporar el efecto de estas variaciones. En primer lugar, será necesario identificar qué términos, anteriormente constantes, variarán ahora con la masa del vehículo. En concreto, las siguientes propiedades deben ser escritas como una función de la cantidad de agua en los tanques:

- Masa $(m)$. 


\begin{tabular}{|c|c|c|c|c|}
\hline & MBT 2 & MBT 3 & MBT 4 & MBT 5 \\
\hline$A_{h}$ & 0,191 & 0,191 & 0,191 & 0,191 \\
\hline$A_{v}$ & 0,0177 & 0,0177 & 0,0177 & 0,0177 \\
\hline$C_{h}$ & 0,7 & 0,7 & 0,7 & 0,7 \\
\hline$H_{t k}$ & 5 & 5 & 6,2 & 6,2 \\
\hline$p_{F 0}$ & $2,5 \cdot 10^{7}$ & $2,5 \cdot 10^{7}$ & $2,5 \cdot 10^{7}$ & $2,5 \cdot 10^{7}$ \\
\hline$T_{F 0}$ & 293 & 293 & 293 & 293 \\
\hline$V_{B 0}$ & 0,001 & 0,001 & 0,001 & 0,001 \\
\hline$V_{B B}$ & 21,4 & 21,4 & 22,9 & 22,9 \\
\hline$V_{F}$ & 0,8 & 0,8 & 0,8 & 0,8 \\
\hline$x_{b}$ & $-28,6$ & $-28,6$ & 23 & 23 \\
\hline$y_{b}$ & 1,2 & $-1,2$ & 1,7 & $-1,7$ \\
\hline$z_{b}$ & 0,595 & 0,595 & 0,975 & 0,975 \\
\hline$z_{h}$ & $-2,895$ & $-2,895$ & $-3,897$ & $-3,897$ \\
\hline$z_{t}$ & 2,105 & 2,105 & 2,303 & 2,303 \\
\hline$z_{v}$ & 2,705 & 2,705 & 2,705 & 2,705 \\
\hline
\end{tabular}

Tabla 3.2: Características geométricas de los tanques de lastre.

- Peso $(W)$.

- Momentos y productos de inercia $\left(I_{x}, I_{y}, I_{z}, I_{x y}, \ldots\right)$.

- Coordenadas del centro de gravedad $\left(x_{G}, y_{G}, z_{G}\right)$.

En segundo lugar, puesto que tanto la masa como las componentes del tensor de inercia son ahora variables con el tiempo, en las ecuaciones de conservación del momento lineal y el momento angular, (1.3)-(1.4), aparecerán, junto a los términos de la forma $m \dot{x}, I \dot{x}$, términos de la forma $\frac{d m}{d t} x, \frac{d I}{d t} x$. Como veremos posteriormente, en el contexto de los submarinos tripulados, en los que las variaciones de masa son pequeñas con respecto a la masa total, es habitual despreciar estos últimos términos frente a los primeros (ver [Wat07, BGW09]).

Supongamos que existen $N$ tanques de lastre con centros geométricos localizados en $\left(x_{b i}\right.$, $y_{b i}, z_{b i}$ ) (donde el subíndice $i$ denota el $i$-ésimo tanque de lastre). Llamaremos $m_{0}$ a la masa inicial del submarino (con todos los tanques completamente llenos de agua) y $\Delta m_{i}$ a la masa perdida en el $i$-ésimo tanque, nula con el tanque completamente lleno y máxima con el tanque vacío.

El volumen de agua que ha abandonado el tanque es igual al volumen ocupado por el aire excepto por el volumen de aire inicial, $V_{B 0}$, que depende de la masa de aire inicial, $m_{B 0}$, y la cota inicial. La masa perdida en el $i$-ésimo tanque puede obtenerse multiplicando este 

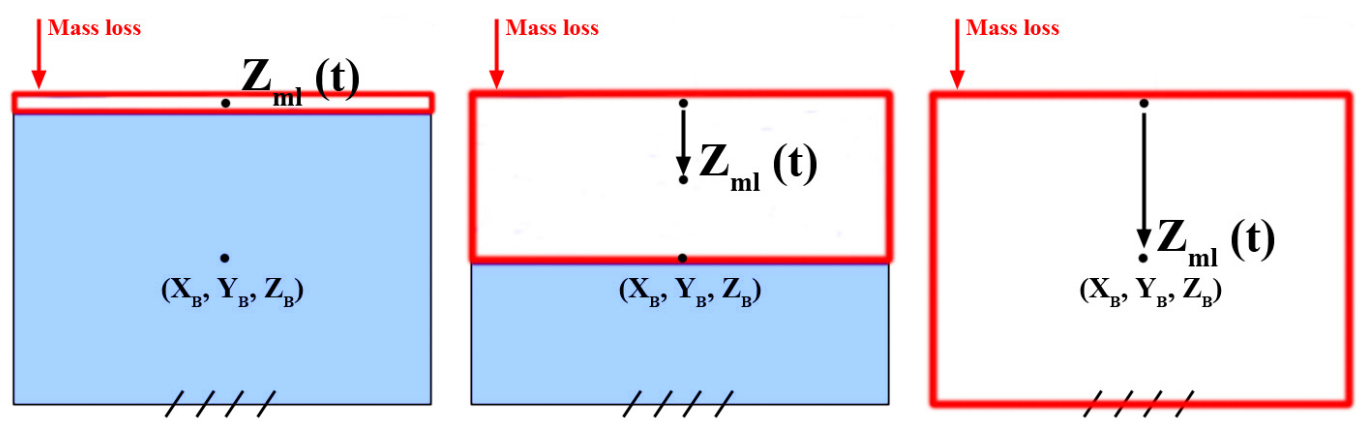

Figura 3.4: Localización de la pérdida de masa.

volumen por la densidad del agua, $\rho$, es decir,

$$
\Delta m_{i}(t)=\rho\left(V_{B i}(t)-V_{B 0}\right)=\rho\left(\frac{m_{B i}(t) R_{g} T_{B}}{p_{B i}(t)}-V_{B 0}\right) .
$$

La masa instantánea del submarino es por lo tanto

$$
m(t)=m_{0}-\sum_{i=1}^{N} \Delta m_{i}(t),
$$

y el peso

$$
W(t)=g\left(m_{0}-\sum_{i=1}^{N} \Delta m_{i}(t)\right),
$$

con $g$ la aceleración de la gravedad.

Para expresar las coordenadas del centro de gravedad y los momentos y productos de inercia en función de la cantidad de agua en los tanques trabajaremos bajo la hipótesis de que las pérdidas de masa pueden suponerse localizadas en puntos $\left(x_{b i}, y_{b i}, z_{m l i}(t)\right)$, donde $z_{m l i}(t)$ es la altura a la que sucede la pérdida de masa en cada uno de los tanques. Esta coordenada varía, como puede verse en la Figura 3.4, desde la parte superior del tanque, cuando éste está completamente lleno, hasta su centro geométrico, $z_{b i}$, cuando está completamente vacío. Esta variación se supone lineal, de tal forma que

$$
z_{m l i}(t)=z_{t i}-\frac{\left(z_{t i}-z_{b i}\right) \Delta m_{i}(t)}{\Delta m_{i, \max }}
$$

donde $z_{t i}$ es la posición de la parte superior del tanque y $\Delta m_{i, \max }$ es el valor máximo de la pérdida de masa, es decir, el valor cuando el tanque está completamente vacío.

Sean $I_{x 0}, I_{y 0}, I_{z 0}, I_{x y 0}, I_{x z 0}, I_{y z 0}$ y $x_{G 0}, y_{G 0}, z_{G 0}$ respectivamente los momentos y productos de inercia y las coordenadas del centro de gravedad iniciales (con todos los tanques completamente llenos), los valores instantáneos vendrán dados por 


$$
\begin{aligned}
& I_{x}(t)=\int\left(y^{2}+z^{2}\right) d m=I_{x 0}-\sum_{i=1}^{N}\left(y_{b i}^{2}+z_{m l i}(t)^{2}\right) \Delta m_{i}(t), \\
& I_{y}(t)=\int\left(x^{2}+z^{2}\right) d m=I_{y 0}-\sum_{i=1}^{N}\left(x_{b i}^{2}+z_{m l i}(t)^{2}\right) \Delta m_{i}(t), \\
& I_{z}(t)=\int\left(x^{2}+y^{2}\right) d m=I_{z 0}-\sum_{i=1}^{N}\left(x_{b i}^{2}+y_{b i}^{2}\right) \Delta m_{i}(t), \\
& I_{x y}(t)=\int x y d m=I_{x y 0}-\sum_{i=1}^{N} x_{b i} y_{b i} \Delta m_{i}(t), \\
& I_{x z}(t)=\int x z d m=I_{x z 0}-\sum_{i=1}^{N} x_{b i} z_{m l i}(t) \Delta m_{i}(t), \\
& I_{y z}(t)=\int y z d m=I_{y z 0}-\sum_{i=1}^{N} y_{b i} z_{m l i}(t) \Delta m_{i}(t),
\end{aligned}
$$

y las coordenadas del centro de gravedad

$$
\begin{aligned}
& x_{G}(t)=\frac{1}{m_{0}-\sum_{i=1}^{N} \Delta m_{i}(t)}\left(m_{0} x_{G 0}-\sum_{i=1}^{N} x_{b i} \Delta m_{i}(t)\right) \\
& y_{G}(t)=\frac{1}{m_{0}-\sum_{i=1}^{N} \Delta m_{i}(t)}\left(m_{0} y_{G 0}-\sum_{i=1}^{N} y_{b i} \Delta m_{i}(t)\right) \\
& z_{G}(t)=\frac{1}{m_{0}-\sum_{i=1}^{N} \Delta m_{i}(t)}\left(m_{0} z_{G 0}-\sum_{i=1}^{N} z_{m l i}(t) \Delta m_{i}(t)\right)
\end{aligned}
$$

En cuanto a los términos de la forma $\frac{d m}{d t} x, \frac{d I}{d t} x$, de (3.23)-(3.26) y (3.22) se obtiene que la variación con el tiempo de la masa y los productos de inercia, por ejemplo $I_{z}$, vale

$$
\begin{aligned}
\frac{d m(t)}{d t} & =\rho R_{g} T_{B} \sum_{i=1}^{N}\left(\frac{m_{B i}(t) \dot{p}_{B i}(t)}{p_{B i}^{2}(t)}-\frac{\dot{m}_{B i}(t)}{p_{B i}(t)}\right) \\
\frac{d I_{z}(t)}{d t} & =\rho R_{g} T_{B} \sum_{i=1}^{N}\left(x_{b i}^{2}+y_{b i}^{2}\right)\left(\frac{m_{B i}(t) \dot{p}_{B i}(t)}{p_{B i}^{2}(t)}-\frac{\dot{m}_{B i}(t)}{p_{B i}(t)}\right) .
\end{aligned}
$$

La máxima velocidad de variación de la masa se producirá al comienzo del proceso de soplado, cuando el flujo desde la botella es máximo y la entrada del aire en el tanque produce una sobrepresión. El valor de esta sobrepresión es mayor a menor cota y por tanto menor presión exterior. Los experimentos realizados permiten estimar la sobrepresión en alrededor de un $15 \%$ para una cota de $100 \mathrm{~m}$. Utilizando este valor, que puesto que el flujo desde la botella es máximo, $\dot{m}_{B}=-\dot{m}_{F \max }$, y particularizando para los valores de la Tabla 3.2, podemos estimar la cota superior de la variación de masa en alrededor de los $6 \cdot 10^{3} \mathrm{~kg} / \mathrm{s}$. Puesto que la masa total del vehículo es $m_{0}=2352 \cdot 10^{3} \mathrm{~kg}$ y los momentos de inercia iniciales valen $I_{x 0}=16390 \cdot 10^{3}, I_{y 0}=659770 \cdot 10^{3}, I_{z 0}=659770 \cdot 10^{3} \mathrm{kgm}^{2}$ (Tabla 1.3), incluso si 
las derivadas de las variables de estado son un orden de magnitud menores que éstas, los términos de la forma $\frac{d m}{d t} x, \frac{d I}{d t} x$ serán alrededor de dos órdenes de magnitud inferiores a los de la forma $m \dot{x}, I \dot{x}$.

De este modo, la variación de la masa con el tiempo puede ser despreciada, considerándose únicamente de forma quasi-estacionaria mediante la actualización, en cada instante de tiempo, de los valores de masa, peso, momentos de inercia y coordenadas del centro de gravedad. Bajo esta hipótesis, la sustitución de (3.23)-(3.27) en las ecuaciones del movimiento completa nuestro modelo de masa variable. 



\section{Forma final del modelo completo. Análisis matemático}

Tras tratar en capítulos anteriores los distintos componentes del modelo matemático, el propósito del presente capítulo es abordar éste en su conjunto. La Sección 4.1 expone brevemente cómo encajan las distintas piezas para conformar el modelo completo y propone su forma compacta, que será la empleada en adelante. En la Sección 4.2 se realiza un análisis matemático del modelo, demostrando la existencia y unicidad de solución para la ley de estado. Por último, en la Sección 4.3 se analizan las limitaciones conocidas del modelo y se proponen posibles mejoras.

\subsection{Modelo completo en forma compacta}

De acuerdo con lo expuesto en capítulos anteriores, el modelo matemático propuesto para el movimiento de un vehículo submarino teniendo en cuenta el posible soplado/ventilación de los tanques de lastre se compone de un conjunto de ecuaciones (3.19)-(3.21) por cada uno de los tanques considerados más las ecuaciones cinemáticas y dinámicas (1.7)-(1.18) incorporando la dependencia de la cantidad de agua en los tanques de los parámetros descritos en la Sección 3.7 mediante (3.22)-(3.27). Adicionalmente, si se desea incorporar el efecto de la compresibilidad de los materiales y/o la variación de la densidad del agua, la flotabilidad vendrá dada por (2.30), mientras que si desea considerar el efecto del oleaje, se incorporarán en las ecuaciones correspondientes los esfuerzos causados por el oleaje según se describe en la Sección 2.1.2. 
De este modo, las variables de estado pueden expresarse en forma vectorial como

$$
\mathbf{x}(t)=\left[\left[m_{F_{i}}(t), m_{B_{i}}(t), p_{B_{i}}(t)\right]_{1 \leq i \leq N}, \eta(t)^{T}, \nu(t)^{T}\right]^{T}
$$

donde $N$ es el número de tanques de lastre, $\eta=[x, y, z, \phi, \theta, \psi]^{T}$ es el vector con la posición y orientación del vehículo y $\nu=[u, v, w, p, q, r]^{T}$ son las velocidades lineales y angulares. El vector de estado $\mathbf{x}$, por tanto, pertenece a $\mathbb{R}^{3 N+12}$.

El vector de control, por su parte, puede escribirse

$$
\mathbf{u}(t)=\left[s_{i}(t), \bar{s}_{i}(t)\right]_{1 \leq i \leq N}^{T}
$$

siendo $s_{i}$ y $\bar{s}_{i}$, respectivamente, la apertura de las válvulas de soplado y ventilación del $i$-ésimo tanque. Por tanto, $\mathbf{u} \in \mathbb{R}^{2 N}$.

La ley de estado puede escribirse de forma compacta como

$$
\mathbf{A}(\mathbf{x}(t)) \dot{\mathbf{x}}(t)=\mathbf{f}(t, \mathbf{x}(t), \mathbf{u}(t))
$$

donde $\mathbf{A}(\mathbf{x}(t)) \in \mathcal{M}^{(3 N+12) \times(3 N+12)}$ y $\mathbf{f}(t, \mathbf{x}(t), \mathbf{u}(t)) \in \mathbb{R}^{3 N+12}$ para todo $t>0$. En la Sección 4.2 se analizará en detalle la estructura de $\mathbf{A}$ y $\mathbf{f}$.

Puesto que nuestro objetivo es explorar el posible uso del soplado/ventilación como un mecanismo de control que permita mejorar la maniobrabilidad del vehículo, habitualmente consideraremos el vector de control, como acabamos de ver, compuesto únicamente por las aperturas de las válvulas de soplado y ventilación. Las deflexiones de los timones y la velocidad de giro de la hélice, variables que tradicionalmente son empleadas para el control del vehículo, tomaran, por su parte, valores prefijados, $\delta_{r}(t), \delta_{b}(t), \delta_{s}(t), n(t)$; de ahí la dependencia explícita de $\mathbf{f}$ con el tiempo. En términos matemáticos, asumiremos que $\delta_{b}, \delta_{s}, \delta_{r}$, $n \in L^{\infty}\left(0, t_{f} ; K\right)$, donde

$$
K=\left[-\frac{5 \pi}{36}, \frac{5 \pi}{36}\right] \times\left[-\frac{5 \pi}{36}, \frac{5 \pi}{36}\right] \times\left[-\frac{7 \pi}{36}, \frac{7 \pi}{36}\right] \times[0,2,5]
$$

es el conjunto de valores admisibles para estas variables.

\subsection{Análisis matemático}

Una vez obtenido el conjunto de las ecuaciones de estado en su forma final, el objetivo de esta sección es estudiar algunas propiedades matemáticas de la ley de estado. La Sección 4.2.1 muestra que tanto los controles como las variables de estado están acotados, mientras que en la Sección 4.2.2 se demuestra que la ley de estado está bien planteada. 


\subsection{1. $\quad$ El vector de estado es acotado}

Veremos en esta sección que el vector de estado $\mathbf{x}(t)$ definido por (4.1) toma siempre valores acotados.

En cuanto a las variables de estado relativas a los tanques de lastre, puesto que la presión exterior a una cierta cota es la suma de la presión atmosférica y el peso de la columna de agua sobre el submarino, incluso si la presión en el interior del tanque es ligeramente inferior a la exterior y el vehículo está próximo a la superficie, podemos asumir sin riesgo que la presión en el tanque será siempre mayor que la presión atmosférica, es decir, $p_{B_{i}} \geq p_{B}^{-}=p_{a t m}$. Pese a que no es tan sencillo obtener una cota superior para la presión en el tanque, es fácil ver que ésta se reducirá siempre a valores finitos, lo que justifica suponer que $p_{B_{i}} \leq p_{B}^{+}<+\infty$. Es también inmediato ver que el límite superior para la cantidad de aire en la botella y el tanque es la masa inicial de aire en la botella. Hemos considerado por hipótesis que siempre existe una cantidad de aire residual en el tanque, $m_{B_{0}}$, por lo que $m_{B_{i}} \geq m_{B}^{-}=m_{B_{0}}>0$. Como dijimos anteriormente, la presión en el tanque no puede ser menor que $p_{a t m}$. Puesto que el aire fluye debido a la diferencia de presión entre botella y tanque, la presión en la botella tendrá esta misma cota inferior. Por tanto, haciendo uso de la ecuación de los gases perfectos y (3.7), puede obtenerse una cota inferior para la masa de aire en la botella, $m_{F_{i}} \geq m_{F}^{-}>0$. Resumiendo, podemos asumir los siguientes límites

$$
\left\{\begin{array}{l}
0<m_{F}^{-} \leq m_{F_{i}} \leq m_{F}^{+}<+\infty \\
0<m_{B}^{-} \leq m_{B_{i}} \leq m_{B}^{+}<+\infty \\
0<p_{B}^{-} \leq p_{B_{i}} \leq p_{B}^{+}<+\infty
\end{array}\right.
$$

Respecto a los ángulos de Euler, puesto que trabajamos con submarinos tripulados, típicamente

$$
-\frac{\pi}{4}<\phi<\frac{\pi}{4}, \quad-\frac{\pi}{4}<\theta<\frac{\pi}{4}, \quad 0<\psi<2 \pi .
$$

Debido a la naturaleza finita del océano, la posición del vehículo, $(x, y, z)$, estará también limitada al interior de un rectángulo acotado. Finalmente, la física del problema impone también restricciones sobre el resto de componentes (velocidades lineales $(u, v, w)$ y angulares $(p, q, r))$.

En conclusión, podemos asumir que el conjunto en el que la variable de estado $\mathbf{x}(t)$ toma valores, que en adelante denotaremos por $\Omega$, es acotado. Escribiremos $\mathbf{x}(t) \in \Omega$.

Por su parte, dado que las variables $s_{i}, \bar{s}_{i}$ toman, como vimos en la Sección 3.6, valores entre cero y uno, es inmediato ver que el vector de control $\mathbf{u}(t)$ definido por (4.2) verifica

$$
\mathbf{u}(t) \in[0,1]^{2 N} \quad \forall t \geq 0 .
$$

En lo sucesivo denotaremos por $\mathcal{K}=[0,1]^{2 N}$ el conjunto en el que toma valores el vector de control, de modo que éste satisface $\mathbf{u} \in L^{\infty}(0,+\infty ; \mathcal{K})$. 


\subsubsection{La ley de estado está bien planteada}

Demostraremos que para todo estado inicial $\mathbf{x}^{0} \in \Omega$ y todo control admisible $\mathbf{u}(t) \in$ $L^{\infty}(0,+\infty ; \mathcal{K})$ existe una única solución de $(4.3)$ partiendo de $\mathbf{x}^{0}$, y cumpliendo $\mathbf{x}(t) \in \Omega$, definida en un intervalo $0 \leq t \leq t_{f}$ donde $t_{f}=t_{f}\left(\mathbf{x}^{0}\right)$ sólo depende de la condición inicial. Puesto que la ley de estado viene expresada en forma implícita, sin embargo, no es posible aplicar los resultados habituales. Mostraremos por tanto, en primer lugar, que la matriz A es invertible, de modo que la ley de estado puede expresarse en forma explícita como

$$
\dot{\mathbf{x}}(t)=\mathbf{A}(\mathbf{x}(t))^{-1} \mathbf{f}(t, \mathbf{x}(t), \mathbf{u}(t)) .
$$

\section{La matriz A es invertible}

Es sencillo ver que para todo $\mathbf{x} \in \Omega$, la matriz $\mathbf{A}(\mathbf{x})$ tiene la forma

$$
\mathbf{A}(\mathbf{x})=\left[\begin{array}{ccc}
\mathbf{B V}(\mathbf{x}) & \mathbf{0}_{3 N \times 6} & \mathbf{0}_{3 N \times 6} \\
\mathbf{0}_{6 \times 3 N} & I_{6} & \mathbf{0}_{6 \times 6} \\
\mathbf{0}_{6 \times 3 N} & \mathbf{0}_{6 \times 6} & \mathbf{M}(\mathbf{x})
\end{array}\right]
$$

donde $\mathbf{B V}(\mathbf{x})$ es la submatriz asociada con las ecuaciones relativas a los tanques de lastre y $\mathbf{M}(\mathbf{x})$ es la matriz de masas, en este caso variable. Adicionalmente, $\mathbf{B V}(\mathbf{x})$ es una matriz $3 N \times 3 N$ estructurada en bloques diagonales $3 \times 3$

$$
\mathbf{B V}(\mathbf{x})=\left[\begin{array}{ccc}
\mathbf{B V}_{1}(\mathbf{x}) & \cdots & \mathbf{0}_{3 \times 3} \\
\vdots & \ddots & \vdots \\
\mathbf{0}_{3 \times 3} & \cdots & \mathbf{B V}_{N}(\mathbf{x})
\end{array}\right]
$$

donde, para $1 \leq i \leq N$,

$$
\mathbf{B V}_{i}(\mathbf{x})=\left[\begin{array}{rrc}
1 & 0 & 0 \\
1 & 1 & 0 \\
0 & -1 & \frac{m_{B_{i}}}{p_{B_{i}}}
\end{array}\right]
$$

con $\operatorname{det}\left(\mathbf{B V}_{i}(\mathbf{x})\right)=\frac{m_{B_{i}}}{p_{B_{i}}} \geq \frac{m_{B}^{-}}{p_{B}^{+}}>0$ por (4.4). Así pues, $\mathbf{B} V_{i}(\mathbf{x})$ es invertible con

$$
\mathbf{B V}_{i}(\mathbf{x})^{-1}=\left[\begin{array}{rcc}
1 & 0 & 0 \\
-1 & 1 & 0 \\
\frac{-p_{B_{i}}}{m_{B_{i}}} & \frac{p_{B_{i}}}{m_{B_{i}}} & \frac{p_{B_{i}}}{m_{B_{i}}}
\end{array}\right]
$$

y, por tanto, la matriz $\mathbf{B V}(\mathbf{x})$ completa es también invertible para todo estado admisible $\mathrm{x} \in \Omega$ con

$$
\mathbf{B V}(\mathbf{x})^{-1}=\left[\begin{array}{ccc}
\mathbf{B V}_{1}(\mathbf{x})^{-1} & \cdots & \mathbf{0}_{3 \times 3} \\
\vdots & \ddots & \vdots \\
\mathbf{0}_{3 \times 3} & \cdots & \mathbf{B V}_{N}(\mathbf{x})^{-1}
\end{array}\right]
$$


Resta probar que la matriz de masas $\mathbf{M}$ es invertible. De las ecuaciones dinámicas y (3.23)-(3.27) tenemos que $\mathbf{M}(\mathbf{x})$ puede descomponerse en una parte constante y una variable:

$$
\mathbf{M}(\mathbf{x})=\mathbf{M}_{v}(\mathbf{x})+\mathbf{M}_{c}
$$

donde

$$
\mathbf{M}_{v}(\mathbf{x})=\left[\begin{array}{cc}
m(\mathbf{x}) I_{3} & -\mathbf{S}(\mathbf{x}) \\
\mathbf{S}(\mathbf{x}) & \mathbf{I}(\mathbf{x})
\end{array}\right]
$$

es la parte variable, con

$$
\boldsymbol{S}(\mathbf{x})=\left[\begin{array}{ccc}
0 & -m(\mathbf{x}) z_{G}(\mathbf{x}) & m(\mathbf{x}) y_{G}(\mathbf{x}) \\
m(\mathbf{x}) z_{G}(\mathbf{x}) & 0 & -m(\mathbf{x}) x_{G}(\mathbf{x}) \\
-m(\mathbf{x}) y_{G}(\mathbf{x}) & m(\mathbf{x}) x_{G}(\mathbf{x}) & 0
\end{array}\right]
$$

$\mathrm{e}$

$$
\mathbf{I}(\mathbf{x})=\left[\begin{array}{ccc}
I_{x}(\mathbf{x}) & -I_{x y}(\mathbf{x}) & -I_{z x}(\mathbf{x}) \\
-I_{x y}(\mathbf{x}) & I_{y}(\mathbf{x}) & -I_{y z}(\mathbf{x}) \\
-I_{z x}(\mathbf{x}) & -I_{y z}(\mathbf{x}) & I_{z}(\mathbf{x})
\end{array}\right]
$$

el tensor de inercia, y $\mathbf{M}_{c}$ la matriz de masa añadida

$$
\left[\begin{array}{cccccc}
-\frac{\rho}{2} l^{3} X_{\dot{u}}^{\prime} & 0 & 0 & 0 & 0 & 0 \\
0 & -\frac{\rho}{2} l^{3} Y_{\dot{v}}^{\prime} & 0 & -\frac{\rho}{2} l^{4} Y_{\dot{p}}^{\prime} & 0 & -\frac{\rho}{2} l^{4} Y_{\dot{r}}^{\prime} \\
0 & 0 & -\frac{\rho}{2} l^{3} Z_{\dot{w}}^{\prime} & 0 & -\frac{\rho}{2} l^{4} Z_{\dot{q}}^{\prime} & 0 \\
0 & -\frac{\rho}{2} l^{4} K_{\dot{v}}^{\prime} & 0 & -\frac{\rho}{2} l^{5} K_{\dot{p}}^{\prime} & 0 & -\frac{\rho}{2} l^{5} K_{\dot{r}}^{\prime} \\
0 & 0 & -\frac{\rho}{2} l^{4} M_{\dot{w}}^{\prime} & 0 & -\frac{\rho}{2} l^{5} M_{\dot{q}}^{\prime} & 0 \\
0 & -\frac{\rho}{2} l^{4} N_{\dot{v}}^{\prime} & 0 & -\frac{\rho}{2} l^{5} N_{\dot{p}}^{\prime} & 0 & -\frac{\rho}{2} l^{5} N_{\dot{r}}^{\prime}
\end{array}\right]
$$

Es usual en la literatura sobre vehículos submarinos (véase, por ejemplo, [Fos94, Property 2.4]) considerar que $\mathbf{M}_{c}$ es una matriz simétrica definida positiva y por tanto invertible. En el caso concreto del prototipo en consideración (ver Tabla 1.5) esta matriz no es simétrica, aunque puede comprobarse que es invertible.

En caso de que todos los tanques estén completamente llenos, la matriz de masas tomará el valor $\mathbf{M}_{0}+\mathbf{M}_{c}$, siendo $\mathbf{M}_{0}$ la parte variable de la matriz de masas, $\mathbf{M}_{v}(\mathbf{x})$, particularizada para esta situación,

$$
\mathbf{M}_{\mathbf{0}}=\left[\frac{m_{0} I_{3}\left[\begin{array}{ccc}
0 & z_{G 0} & -y_{G 0} \\
-z_{G 0} & 0 & x_{G 0} \\
y_{G 0} & -x_{G 0} & 0
\end{array}\right]}{m_{0}\left[\begin{array}{ccc}
0 & -z_{G 0} & y_{G 0} \\
z_{G 0} & 0 & -x_{G 0} \\
-y_{G 0} & x_{G 0} & 0
\end{array}\right]} \frac{\mathbf{I}_{\mathbf{0}}}{]}\right] .
$$


Esta matriz es simétrica y definida positiva ([Fos94, Property 2.2]). Por tanto, cuando también $\mathbf{M}_{c}$ se asume simétrica y definida positiva, la matriz de masas $\mathbf{M}_{0}+\mathbf{M}_{c}$ es invertible puesto que es también simétrica y definida positiva. Si esto no es así, como en el presente caso, la invertibilidad de $\mathbf{M}_{0}+\mathbf{M}_{c}$ debe ser verificada para los valores numéricos particulares en consideración. Basta la sustitución de los valores recogidos en las tablas 1.3 y 1.5 y el cálculo de det $\left(\mathbf{M}_{0}+\mathbf{M}_{c}\right)$ para comprobar que esta matriz es, en nuestro caso, invertible.

Si la matriz de masas para tanques completamente llenos es invertible, y puesto que las variaciones de masa son pequeñas respecto a la masa total, $\mathbf{M}(\mathbf{x})$ tomará valores cercanos a $\mathbf{M}_{0}+\mathbf{M}_{c}$, por lo que la invertibilidad de $\mathbf{M}(\mathbf{x})$ podrá deducirse a partir de la $\mathbf{M}_{0}+\mathbf{M}_{c}$. En términos matemáticos, la invertibilidad de $\mathbf{M}(\mathbf{x})$ se deriva del siguiente Lema.

Lema 4.1. Sea $\mathbf{D} \in \mathcal{M}^{m \times m}$ una matriz invertible y sea $\boldsymbol{\Delta} \in \mathcal{M}^{m \times m}$ una matriz cuadrada tal que

$$
\left\|\mathbf{D}^{-1} \boldsymbol{\Delta}\right\|=\sup \left\{\left|\mathbf{D}^{-1} \boldsymbol{\Delta} \mathbf{y}\right|:|\mathbf{y}|=1\right\}<1
$$

donde $|\cdot|$ denota la norma Euclídea en $\mathbb{R}^{m}$. Entonces $\mathbf{D}+\boldsymbol{\Delta}$ es invertible con

$$
(\mathbf{D}+\boldsymbol{\Delta})^{-1}=\sum_{n=0}^{\infty}(-1)^{n}\left(\mathbf{D}^{-1} \boldsymbol{\Delta}\right)^{n} \mathbf{D}^{-1}
$$

El cálculo de la norma de una matriz no es en general sencillo. El siguiente Lema proporciona una útil aproximación.

Lema 4.2. Sea $\mathbf{D}=\left[d_{i j}\right] \in \mathcal{M}^{m \times m}$ una matriz cuadrada. Entonces

$$
\|\mathbf{D}\| \leq \sqrt{\sum_{i, j} d_{i j}^{2}} .
$$

El término de la derecha en (4.15) recibe el nombre de norma de Frobenius de $\mathbf{D},\|\mathbf{D}\|_{F}$.

Sea $\mathbf{x} \in \Omega$ un estado admisible cualquiera. Puesto que $\mathbf{M}(\mathbf{x})=\left(\mathbf{M}_{0}+\mathbf{M}_{c}\right)+\left(\mathbf{M}_{v}(\mathbf{x})-\mathbf{M}_{0}\right)$ con $\mathbf{M}_{0}+\mathbf{M}_{c}$ invertible, por el Lema 4.1 basta con verificar que

$$
\left\|\left(\mathbf{M}_{0}+\mathbf{M}_{c}\right)^{-1}\left(\mathbf{M}_{v}(\mathbf{x})-\mathbf{M}_{0}\right)\right\|<1
$$

para probar la invertibilidad de $\mathbf{M}(\mathbf{x})$. Además, el Lema 4.1 garantiza también que

$$
\mathbf{M}(\mathbf{x})^{-1}=\sum_{n=0}^{\infty}(-1)^{n}\left(\left(\mathbf{M}_{0}+\mathbf{M}_{c}\right)^{-1}\left(\mathbf{M}_{v}(\mathbf{x})-\mathbf{M}_{0}\right)\right)^{n}\left(\mathbf{M}_{0}+\mathbf{M}_{c}\right)^{-1}
$$

En conclusión, $\mathbf{M}(\mathbf{x})$, y por tanto $\mathbf{A}(\mathbf{x})$, es invertible para todo estado $\mathbf{x} \in \Omega$ si $\mathbf{M}_{0}+\mathbf{M}_{c}$ es invertible y la variación en la masa debida a las operaciones de soplado y ventilación es suficientemente pequeña. De forma precisa, el siguiente teorema nos garantiza la invertibilidad de $\mathbf{M}(\mathbf{x})$ si se verifica

$$
\Delta m^{+}<\frac{\lambda_{\min }}{\sqrt{3+4 N \sum_{i=1}^{N}\left|\mathbf{c}_{i}\right|^{2}+\frac{9}{2} \sum_{i=1}^{N}\left|\mathbf{c}_{i}\right|^{4}}}
$$


donde $\Delta m^{+}=\sum_{i=1}^{N} \Delta m_{i, \max }=\rho \sum_{i=1}^{N}\left(V_{B B i}-V_{B 0}\right)$ es la máxima variación en la masa debida al soplado/ventilación, $\lambda_{\text {min }}>0$ es el menor valor singular ${ }^{1}$ de la matriz $\mathbf{M}_{0}+\mathbf{M}_{c} \mathrm{y}$ $\mathbf{c}_{i}$ es el centro geométrico del $i$-ésimo tanque de lastre.

Teorema 4.3. Si la matriz $\mathbf{M}_{0}+\mathbf{M}_{c}$ es invertible y se verifica la desigualdad (4.18), entonces la aplicación matricial $\mathbf{A}: \Omega \longrightarrow \mathcal{M}^{(12+3 N) \times(12+3 N)}$ toma valores no singulares, es decir, $\mathbf{A}(\mathbf{x})$ es invertible para todo $\mathbf{x} \in \Omega$ con

$$
\mathbf{A}(\mathbf{x})^{-1}=\left[\begin{array}{ccc}
\mathbf{B V}(\mathbf{x})^{-1} & \mathbf{0}_{3 N \times 6} & \mathbf{0}_{3 N \times 6} \\
\mathbf{0}_{6 \times 3 N} & I_{6} & \mathbf{0}_{6 \times 6} \\
\mathbf{0}_{6 \times 3 N} & \mathbf{0}_{6 \times 6} & \mathbf{M}(\mathbf{x})^{-1}
\end{array}\right] .
$$

Además, la aplicación $\mathbf{x} \leadsto \mathbf{A}(\mathbf{x})^{-1}$ es continuamente diferenciable.

Demostración. Para probar que $\mathbf{A}(\mathbf{x})$ es invertible para todo $\mathbf{x} \in \Omega$, basta con ver que (4.16) se satisface uniformemente con respecto a la variable de estado. Por las propiedades de la norma matricial (ver, por ejemplo, [Son90, Appendix A]), tenemos

$$
\begin{aligned}
\left\|\left(\mathbf{M}_{0}+\mathbf{M}_{c}\right)^{-1}\left(\mathbf{M}_{v}(\mathbf{x})-\mathbf{M}_{0}\right)\right\| & \leq\left\|\left(\mathbf{M}_{0}+\mathbf{M}_{c}\right)^{-1}\right\|\left\|\mathbf{M}_{v}(\mathbf{x})-\mathbf{M}_{0}\right\| \\
& =\frac{1}{\lambda_{\min }}\left\|\mathbf{M}_{v}(\mathbf{x})-\mathbf{M}_{0}\right\| .
\end{aligned}
$$

Obtengamos ahora una estimación del supremo de la norma de Frobenius de la matriz variable. En primer lugar, de (3.22):

$$
\left|\sum_{i=1}^{N} \Delta m_{i}(\mathbf{x})\right| \leq \sum_{i=1}^{N} \Delta m_{i, \max }=\rho \sum_{i=1}^{N}\left(V_{B B i}-V_{B 0}\right) .
$$

En cuanto a la variación en las coordenadas del centro de gravedad,

$$
\begin{aligned}
\left|\sum_{i=1}^{N} x_{b i} \Delta m_{i}(\mathbf{x})\right| & \leq\left(\Delta m^{+}\right) \sum_{i=1}^{N}\left|x_{b i}\right|, \\
\left|\sum_{i=1}^{N} y_{b i} \Delta m_{i}(\mathbf{x})\right| & \leq\left(\Delta m^{+}\right) \sum_{i=1}^{N}\left|y_{b i}\right|, \\
\left|\sum_{i=1}^{N} z_{m l i}(\mathbf{x}) \Delta m_{i}(\mathbf{x})\right| & \leq\left(\Delta m^{+}\right) \sum_{i=1}^{N}\left|z_{b i}\right|,
\end{aligned}
$$

con $\left(x_{b i}, y_{b i}, z_{b i}\right)$ las coordenadas del centro geométrico del $i$-ésimo tanque (ver Figura 3.4). Finalmente, de (3.26) y (4.20)

$$
\left|I_{x}(\mathbf{x})-I_{x 0}\right|=\left|\sum_{i=1}^{N}\left(y_{b i}^{2}+z_{m l i}^{2}\right) \Delta m_{i}(\mathbf{x})\right| \leq\left(\Delta m^{+}\right) \sum_{i=1}^{N}\left(y_{b i}^{2}+z_{b i}^{2}\right)
$$

\footnotetext{
${ }^{1}$ Se definen los valores singulares de una matriz $A$ como las raíces cuadradas de los valores propios de la matriz $A^{a} A$, donde $A^{a}$ es la matriz adjunta de $A$.
} 
y, de forma análoga,

$$
\begin{aligned}
& \left|I_{y}(\mathbf{x})-I_{y 0}\right| \leq\left(\Delta m^{+}\right) \sum_{i=1}^{N}\left(x_{b i}^{2}+z_{b i}^{2}\right) \\
& \left|I_{z}(\mathbf{x})-I_{z 0}\right| \leq\left(\Delta m^{+}\right) \sum_{i=1}^{N}\left(x_{b i}^{2}+y_{b i}^{2}\right)
\end{aligned}
$$

y

$$
\begin{aligned}
& \left|I_{x y}(\mathbf{x})\right| \leq\left(\Delta m^{+}\right) \sum_{i=1}^{N}\left|x_{b i}\right|\left|y_{b i}\right|, \\
& \left|I_{x z}(\mathbf{x})\right| \leq\left(\Delta m^{+}\right) \sum_{i=1}^{N}\left|x_{b i}\right|\left|z_{b i}\right|, \\
& \left|I_{y z}(\mathbf{x})\right| \leq\left(\Delta m^{+}\right) \sum_{i=1}^{N}\left|y_{b i}\right|\left|z_{b i}\right| .
\end{aligned}
$$

Por tanto, a partir del Lema 4.2, y las estimaciones (4.20)-(4.27), se obtiene que, para cualquier $\mathbf{x} \in \Omega$,

$$
\left\|\mathbf{M}_{v}(\mathbf{x})-\mathbf{M}_{0}\right\| \leq\left(\Delta m^{+}\right) \sqrt{3+4 N\left(\sum_{i=1}^{N} x_{b i}^{2}+y_{b i}^{2}+z_{b i}^{2}\right)+\frac{9}{2}\left(\sum_{i=1}^{N} x_{b i}^{2}+y_{b i}^{2}+z_{b i}^{2}\right)^{2}}
$$

donde se han usado las relaciones $\left(\sum_{i=1}^{N} a_{i}\right)^{2} \leq N\left(\sum_{i=1}^{N} a_{i}^{2}\right)$ y $a b \leq\left(a^{2}+b^{2}\right) / 2$. Combinando esta desigualdad con (4.18), obtenemos la relación deseada (4.16). Finalmente, de (4.10) $\mathrm{y}$ (4.17) se obtiene que $\mathbf{x} \leadsto \mathbf{A}(\mathbf{x})^{-1}$ es continuamente diferenciable, lo que completa la demostración.

En el caso del submarino $\mathrm{P}-650, \mathbf{M}_{0}+\mathbf{M}_{c}$ es, como se dijo con anterioridad, invertible. Además, $\lambda_{\min }=1,54 \cdot 10^{12} \mathrm{y}$ por tanto

$$
\frac{\left(\Delta m^{+}\right) \sqrt{3+4 N\left(\sum_{i=1}^{N} x_{b i}^{2}+y_{b i}^{2}+z_{b i}^{2}\right)+\frac{9}{2}\left(\sum_{i=1}^{N} x_{b i}^{2}+y_{b i}^{2}+z_{b i}^{2}\right)^{2}}}{\lambda_{\min }}=1,73 \cdot 10^{-4},
$$

lo que nos garantiza que, en nuestro caso, $\mathbf{A}(\mathbf{x})$ es, efectivamente, invertible.

\section{La ley de estado está bien planteada}

En caso de que la matriz $\mathbf{A}(\mathbf{x})$ sea invertible, la ley de estado puede escribirse en la forma explícita (4.5). Si consideramos la función

$$
\mathbf{g}:\left[0, t_{f}\right] \times \Omega \longrightarrow \mathbb{R}^{3 N+12}
$$




$$
\mathbf{g}(t, \mathbf{x}, \mathbf{u}(t))=\mathbf{A}(\mathbf{x})^{-1} \mathbf{f}(t, \mathbf{x}, \mathbf{u}(t)),
$$

entonces (4.5) puede, por simplicidad, escribirse como

$$
\dot{\mathbf{x}}(t)=\mathbf{g}(t, \mathbf{x}(t), \mathbf{u}(t)) .
$$

Sea $\mathbf{u} \in L^{\infty}(0,+\infty ; \mathcal{K})$ (ver Sección 4.2.1) un control admisible, para cada $\mathbf{u}$ fijo, las funciones $\mathbf{f}$ y $\mathbf{g}$ en (4.3) y (4.30) pueden expresarse como $\mathbf{f}(t, \mathbf{x}), \mathbf{g}(t, \mathbf{x})$. El propósito de esta sección es probar que (4.30) tiene solución para cualquier estado inicial $\mathbf{x}^{0}$. Comenzamos por recordar la teoría clásica sobre la materia. Se remite a [Son90, Appendix C] para mayor detalle.

Entendemos por solución (en el sentido de Carathéodory) una función absolutamente continua $t \leadsto \mathbf{x}(t) \in \Omega$, definida en un intervalo $I=\left[0, t_{f}\right]$, que satisface la ecuación integral

$$
\mathbf{x}(t)=\mathbf{x}^{0}+\int_{0}^{t} \mathbf{g}(s, \mathbf{x}(s)) d s, \quad \text { para todo } t \in\left[0, t_{f}\right] .
$$

Como es sabido, si g satisface las condiciones (H1)-(H4) a continuación, podemos asegurar la existencia de una única solución maximal de (4.30) para cualquier estado inicial:

(H1) Para todo $\mathbf{x} \in \Omega$, la función $\mathbf{g}(\cdot, \mathbf{x}): I \rightarrow \mathbb{R}^{N}$ es medible.

(H2) Para todo $t \in I$, la función $\mathbf{g}(t, \cdot): \Omega \rightarrow \mathbb{R}^{N}$ es continua.

(H3) $\mathbf{g}$ es localmente Lipschitz con respecto a $\mathbf{x}$, es decir, para todo $\mathbf{x}^{0} \in \Omega$ existe un número real $\varepsilon>0$ y una función localmente integrable $\alpha: I \rightarrow \mathbb{R}^{+}$tal que la bola $B_{\varepsilon}\left(\mathbf{x}^{0}\right)$ de radio $\varepsilon$ y centro $\mathbf{x}^{0}$ está contenida en $\Omega$ y

$$
|\mathbf{g}(t, \mathbf{x})-\mathbf{g}(t, \mathbf{y})| \leq \alpha(t)|\mathbf{x}-\mathbf{y}| \text { para todo } t \in I \quad \mathrm{y} \mathbf{x}, \mathbf{y} \in B_{\varepsilon}\left(\mathbf{x}^{0}\right)
$$

(H4) g es localmente integrable con respecto a $t$, es decir, para todo $\mathbf{x}^{0} \in \Omega$ existe una función localmente integrable $\beta: I \rightarrow \mathbb{R}^{+}$tal que

$$
\left|\mathbf{g}\left(t, \mathbf{x}^{0}\right)\right| \leq \beta(t) \quad \text { c.t.p. } t \in I .
$$

Teorema 4.4. Para todo $\mathbf{x}^{0} \in \Omega$ y todo $\mathbf{u} \in L^{\infty}\left(\mathbb{R}^{+} ; \mathcal{K}\right)$ existe una única solución maximal de (4.30) definida en $\left[0, t_{f}\right]$, donde $t_{f}=t_{f}\left(\mathbf{x}^{0}\right)$ sólo depende del estado inicial $\mathbf{x}^{0}$, y es uniforme con respecto al control $\mathbf{u} \in L^{\infty}\left(\mathbb{R}^{+} ; \mathcal{K}\right)$.

Demostración. Cabe destacar, en primer lugar, que gracias al Teorema 4.3 podemos restringir nuestro análisis al término de la derecha en la ley de estado (4.3). Veamos que se verifican las condiciones (H1)-(H4). Puesto que la variable temporal $t$ sólo aparece en las funciones de control $\left(s_{i}(t), \bar{s}_{i}(t)\right), 1 \leq i \leq N$, y, por hipótesis, estas funciones pertenecen a $L^{\infty}\left(\mathbb{R}^{+} ;[0,1]\right)$, es sencillo ver que para todo $\mathbf{x} \in \Omega$, la función $t \leadsto \mathbf{f}(t, \mathbf{x})$ es medible. 
En lo que respecta a la continuidad de la función $\mathbf{f}(t, \cdot)$, sólo las ecuaciones relativas al flujo de aire desde las botellas necesitan de un análisis detallado con el fin de verificar que el tránsito entre los regímenes super y subsónico es continuo. Puede comprobarse que esta condición se cumple siempre y cuando $m_{F_{i}} \geq m_{F}^{-}>0$ y $p_{B_{i}} \geq p_{B}^{-}>0$.

En cuanto a la condición (H4), debido a la forma en que los controles aparecen en la ley de estado, para todo $\mathbf{x}^{0} \in \Omega$ las componentes $f_{i}\left(t, \mathbf{x}^{0}\right), 1 \leq i \leq 3 N+12$, de $\mathbf{f}\left(t, \mathbf{x}^{0}\right)$ están uniformemente acotadas con respecto a $t$, es decir, (4.32) se verifica para una función constante $\beta(t)=\beta \mathrm{y}$, lo que es más importante, esta constante es uniforme con respecto a $\mathbf{u} \in L^{\infty}\left(\mathbb{R}^{+} ; \mathcal{K}\right)$.

Resta comprobar que $\mathbf{f}(t, \mathbf{x})$ es localmente Lipschitz. Consideremos en primer lugar las ecuaciones $f_{i}, i=1+3 j, 0 \leq j \leq N-1$, que modelan el flujo de aire desde las botellas. Teniendo en cuenta las cotas (4.4), es directo ver que las funciones $f_{i}(t, \cdot)$ son continuas y derivables salvo en un número finito de puntos con discontinuidad de salto finito, es decir, $f_{i}(t, \cdot) \in W^{1, \infty}(\Omega)$, y por tanto son Lipschitz. Es importante destacar que las cotas sobre las derivadas parciales de $f_{i}(t, \cdot)$ son uniformes con respecto a $t$, es decir, existe un $L>0$ tal que

$$
\left|\frac{\partial f_{i}}{\partial x_{j}}(t, \mathbf{x})\right| \leq L \quad \text { para todo } \mathbf{x} \in \boldsymbol{\Omega} \text { y uniformemente c.r.a. } t \geq 0 .
$$

De igual modo, las funciones $f_{i}, i=2+3 j, 0 \leq j \leq N-1$, relativas a la variación de la masa de aire en los tanques son continuas y satisfacen una desigualdad de la forma (4.33). Por tanto, son también Lipschitz. Como antes, la constante de Lipschitz es uniforme con respecto a la variable de control.

Considérense ahora las funciones $f_{3+3 j}, 0 \leq j \leq N-1$, relativas a la evolución de la presión en los tanques. Cuando la presión en el tanque se hace igual a la presión exterior, el gradiente de las velocidades en los orificios de entrada explota debido a la presencia de la raíz cuadrada. Para evitar esta situación se ha aproximado la raíz cuadrada en la forma descrita en la Sección 3.5. De este modo, obtenemos de nuevo que estas aplicaciones son Lipschitz.

Las componentes $f_{i}, 3 N+1 \leq i \leq 3 N+6$, corresponden a la transformación de coordenadas entre los sistemas de referencia fijo y móvil. Teniendo en cuenta las restricciones sobre los ángulos de Euler, es inmediato ver que $f_{i} \in C^{\infty}(\Omega), 3 N+1 \leq i \leq 3 N+6$, y por tanto son Lipschitz. Nótese que la variable temporal no aparece en estas funciones.

En cuanto a las restantes $f_{i}, 3 N+7 \leq i \leq 3 N+12$, estas componentes incluyen: (a) términos polinómicos y términos con valor absoluto; todos ellos Lipschitz, (b) términos del tipo $x_{j} \sqrt{x_{j}^{2}+x_{k}^{2}} \mathrm{y}\left|x_{j}\right| \sqrt{x_{j}^{2}+x_{k}^{2}}$ para algunos $18 \leq j \leq 24$. Puesto que estas funciones son continuas y las discontinuidades en sus derivadas son de salto finito, son también Lipschiz. (c) Dado que estamos usando un modelo de masa variable, es necesario prestar atención a los términos en los que aparecen masa, $m$, peso, $W$, centro de gravedad, $\left(x_{G}, y_{G}, z_{G}\right)$ y momentos y productos de inercia, $I_{x}, I_{y}, I_{z}, I_{x y}, \cdots$, puesto que ahora dependen de parte de las componentes de la variable de estado. Considerando las cotas (4.4), es fácil comprobar que 
estas componentes son también Lipschitz. Finalmente, el producto de funciones Lipschitz es también Lipschitz. Como sucedía anteriormente, la variable de control $\mathbf{u}(t)$ no está incluida en estas componentes $f_{i}$ y por tanto las correspondientes constantes de Lipschitz son independientes de $\mathbf{u}(t)$.

El análisis precedente nos permite concluir que para todo $\mathbf{x}^{0} \in \Omega$ y $\mathbf{u} \in L^{\infty}\left(\mathbb{R}^{+} ; \mathcal{K}\right)$ existe un tiempo maximal $t_{f}=t_{f}\left(\mathbf{x}^{0}, \mathbf{u}\right)$ y una única solución maximal definida en $\left[0, t_{f}\left(\mathbf{x}^{0}, \mathbf{u}\right)\right]$. Si nos fijamos en la demostración del teorema [Son90, Th. 36, pp. 347-351], vemos que $t_{f}$ depende de $\mathbf{u}$ a través de funciones $\alpha(t)=\alpha(\mathbf{u}(t))$ y $\beta(t)=\beta(\mathbf{u}(t))$ que aparecen en (4.31) y (4.32). Como vimos antes, sin embargo, estas funciones $\alpha$ y $\beta$ pueden elegirse uniformemente con respecto a $\mathbf{u} \in L^{\infty}\left(\mathbb{R}^{+} ; \mathcal{K}\right)$. En conclusión, $t_{f}=t_{f}\left(\mathbf{x}^{0}\right)$ sólo depende de la condición inicial, lo que completa la demostración.

\subsection{Limitaciones y posibles mejoras}

Desde su primera formulación en 1967, las ecuaciones propuestas por el David Taylor Naval Ship Research and Development Center han mostrado ser capaces de predecir de forma precisa el movimiento de vehículos submarinos en una amplia variedad de situaciones. Este modelo basado en coeficientes presenta, sin embargo, dos limitaciones principales:

a) En primer lugar, algunos fenómenos hidrodinámicos son tratados de forma quasiestacionaria. Como se discute en [BGW09, Wat07], esta aproximación puede no proporcionar resultados del todo precisos ante movimientos bruscos, como los que pueden producirse al realizar un soplado de emergencia de los tanques de lastre.

La aproximación propuesta en [BGW09] consiste en emplear mecánica de fluidos computacional para resolver las ecuaciones de Navier-Stokes en el entorno del vehículo. En el presente caso, esta aproximación no es viable debido al alto coste computacional asociado. Puesto que buscamos diseñar algoritmos de control o realizar simulaciones en la fase de diseño del submarino, necesitaremos ser capaces de resolver la ley de estado en tiempo real o, al menos, en tiempos reducidos. Es interesante, sin embargo, conocer la naturaleza y el alcance de esta posible fuente de error.

b) En segundo lugar, las ecuaciones y, sobre todo, los valores de los coeficientes hidrodinámicos, se obtienen teniendo en mente un vehículo submarino que navega hacia delante con una cierta velocidad de avance, es decir, para ángulos de incidencia pequeños ${ }^{2}$. Por ello, es de esperar que no se obtengan resultados precisos para ángulos de incidencia fuera

\footnotetext{
${ }^{2}$ El ángulo de incidencia, $\Theta=\tan ^{-1}\left(\sqrt{v^{2}+w^{2}} / u\right)$, es el ángulo formado entre el eje $x$ local y el vector velocidad $\mathbf{U}=(u, v, w)$. Puede entenderse como el ángulo con el que el flujo llega al vehículo y es menor cuanto mayor es la velocidad de avance $u$ respecto a las componentes lateral y vertical, $v$ y $w$.
} 
del rango de validez de las ecuaciones $\left(\leq 30^{\circ}\right)$. El efecto del ángulo de incidencia es analizado en [Hic90, Wat07].

Ésta es una limitación relevante, ya que uno de nuestros objetivos principales es el estudio del posible uso de los procesos de soplado y ventilación como un mecanismo de control alternativo en situaciones con baja o nula velocidad de avance. En estas condiciones el ángulo de incidencia puede alcanzar valores cercanos a los $90^{\circ}$. La actualización de las ecuaciones dinámicas y la obtención de coeficientes hidrodinámicos para el movimiento a grandes ángulos de incidencia, sin embargo, excede con mucho el ámbito del presente trabajo. Por otra parte, en el contexto de este estudio preliminar en el que buscamos comparar mecanismos y estrategias de control, la forma clásica de las ecuaciones es suficiente para obtener conclusiones cualitativas. Si un algoritmo muestra ser capaz de controlar el sistema actual con buenos resultados, es de esperar que fuera capaz de controlar un sistema adaptado a altos ángulos de incidencia una vez que se suministraran al algoritmo las correspondientes ecuaciones y coeficientes.

En caso de que se deseara avanzar hacia la implementación de las soluciones propuestas en un vehículo real, resultaría imprescindible el desarrollo de ecuaciones adaptadas a maniobras con baja o nula velocidad.

En cuanto al modelo para el soplado y ventilación de los tanques de lastre, a pesar de las simplificaciones imprescindibles en la elaboración de todo modelo matemático, se ha tratado de dotarlo de la mayor flexibilidad posible, de forma que más detalle pueda ser añadido allí donde fuera necesario. A continuación se enumeran algunos aspectos del modelo que podrían ser mejorables:

a) El modelo asume que los tanques tienen forma prismática regular, lo cual puede no ser cierto para algunos de ellos. En caso de que se desee incorporar al modelo la forma concreta de cada uno de los taques, bastará con introducir la ley de variación de la sección en (3.25) y (3.15).

b) La suposición de que el caudal a través de las válvulas de soplado y ventilación varía linealmente con su grado de apertura puede, como se dijo anteriormente, no ajustarse a la realidad, particularmente cuando las válvulas estén cerca de la posición de cerrado, donde las pérdidas de presión pueden ser considerables, reduciendo el caudal. En caso de soplados de emergencia esta situación tiene poca importancia, puesto que se produce durante un muy reducido espacio de tiempo. En caso de que se utilice el soplado/ventilación de los tanques como mecanismo de control, sin embargo, puede ser un factor relevante, ya que las válvulas pueden ser abiertas y cerradas múltiples veces en un corto espacio de tiempo. En este caso será necesario, como se dijo, tener en cuenta este efecto incorporando las características concretas de las válvulas empleadas. 
En las simulaciones numéricas que llevaremos a cabo en capítulos posteriores, el posible efecto de este fenómeno ha sido mitigado tomando, para ambas válvulas, tiempos de apertura de hasta 5 segundos, es decir, notablemente superiores a los que sería de esperar en las válvulas que fueran finalmente instaladas. De este modo, el rendimiento que se está suponiendo a las válvulas puede considerarse conservador pese a no estar teniéndose en cuenta el efecto del grado de apertura de las válvulas.

c) La expresión (3.16) para el caudal a través del orificio de salida proporciona una solución estacionaria. Es decir, el régimen transitorio es despreciado. De nuevo, en caso de operaciones de soplado o ventilación completas, el error cometido será pequeño debido a la corta duración del régimen transitorio. En caso de que varios ciclos de apertura y cierre de las válvulas se sucedan con rapidez, en cambio, la existencia del régimen transitorio puede ser relevante. Podría ser interesante cuantificar esta relevancia y, en su caso, obtener una expresión para el caudal $q_{B}$ que incorporara este efecto.

d) La suposición de que la temperatura del aire en el tanque es constante e igual a la temperatura del agua, sostenida en el hecho de que durante el soplado el aire entra a gran velocidad mezclándose rápidamente con el agua contenida en el tanque, se ha mantenido independientemente del valor que tomen las aperturas de las válvulas; es decir, se considera cierta tanto para el proceso de soplado como para el de ventilación. En [FGO10], donde se trataron cada uno de estos procesos por separado, el proceso de ventilación fue, por el contrario, considerado adiabático. Los ensayos realizados, sin embargo, muestran que ambas aproximaciones proporcionan resultados prácticamente idénticos. Si se encontrara que esta simplificación es una fuente de error, puede fácilmente introducirse un término correctivo en la expresión de $\bar{\mu}(3.13)$. 



\section{Parte II}

\section{Algoritmos de Control}





\section{Control óptimo de tanques de lastre}

Llamaremos maniobra, en un sentido amplio, a la acción de ir de un estado inicial a otro final o a la de mantener un cierto estado durante un determinado intervalo de tiempo. Así, son maniobras, por ejemplo, cambios de cota o rumbo, el soplado de emergencia de los tanques de lastre para llevar al submarino a la proximidad de la superficie o el mantenimiento de la cota durante un intervalo de tiempo.

El problema de control consiste en encontrar el vector de control u que logra que el vehículo realice la maniobra deseada. En el caso del control óptimo, además, se busca que la maniobra se realice de la mejor forma posible -óptima- en el sentido de minimizar una cierta función coste $J(\mathbf{x}, \mathbf{u})$. Típicamente, este coste cuantifica una combinación de factores como el seguimiento de una trayectoria deseada, la cercanía en tiempo final al estado final buscado o el valor tomado por los controles. En general, el coste puede ser cualquier función que se desee minimizar. En [Ova11, Chapter 5], por ejemplo, se construye una función coste que tiene por objeto minimizar el ruido hidráulico generado durante la maniobra. En nuestro caso limitaremos la función coste a la cercanía al estado final deseado y el seguimiento de una trayectoria dada.

En la Sección 5.1 se formula el problema de control óptimo para el maniobrado del vehículo mediante el soplado y ventilación de los tanques de lastre. En la Sección 5.2 se demuestra la existencia de solución para este problema, mientras que la Sección 5.3 aborda su resolución numérica. 


\subsection{Formulación del problema de control óptimo}

Sea un estado inicial $\mathbf{x}(0)=\mathbf{x}^{0}$, un tiempo final $t_{f}$, una trayectoria deseada $\overline{\mathbf{x}}(t)=\left[\bar{x}_{j}(t)\right]$, $\mathrm{y}$ un estado final deseado $\mathbf{x}^{t_{f}}$. El objetivo es calcular el vector de control, $\mathbf{u}=\mathbf{u}(t)$, que es capaz de llevar el sistema del estado inicial $\mathbf{x}^{0}$ al estado final $\mathbf{x}^{t_{f}}$ (o las proximidades de éste) en un tiempo $t_{f}$ siguiendo tan cerca como sea posible la trayectoria $\overline{\mathbf{x}}(t)$, cumpliendo a la vez la ley de estado (4.3) y ciertas restricciones sobre el control y el estado. En términos matemáticos tenemos el problema de Bolza

$$
\begin{cases}\text { Minimizar en } \mathbf{u}: & J(\mathbf{u})=\Phi\left(\mathbf{x}\left(t_{f}\right), \mathbf{x}^{t_{f}}\right)+\int_{0}^{t_{f}} F(t, \mathbf{x}(t)) d t \\ \text { sujeto a } & \\ & \mathbf{A}(\mathbf{x}(t)) \dot{\mathbf{x}}(t)=\mathbf{f}(t, \mathbf{x}(t), \mathbf{u}(t)) \\ & \mathbf{x}(0)=\mathbf{x}^{0} \in \Omega \\ & 0 \leq s_{i}(t), \bar{s}_{i}(t) \leq 1,1 \leq i \leq N, \mathbf{y} \quad \mathbf{x}(t) \in \Omega,\end{cases}
$$

donde $\Omega$ es el conjunto en el que toma valores la variable de estado descrito en la Sección 4.2.1 $\mathrm{y}$

$$
\begin{gathered}
\Phi\left(\mathbf{x}\left(t_{f}\right), \mathbf{x}^{t_{f}}\right)=\sum_{j=1}^{3 N+12} \alpha_{j}\left(x_{j}\left(t_{f}\right)-x_{j}^{t_{f}}\right)^{2} \\
F(t, \mathbf{x}(t))=\sum_{j=1}^{3 N+12} \beta_{j}\left(\mathbf{x}_{j}(t)-\overline{\mathbf{x}}_{j}(t)\right)^{2} .
\end{gathered}
$$

El primero de estos términos es una medida de la cercanía del estado en tiempo final al estado final buscado, $\mathbf{x}^{t_{f}}$, mientras que el segundo mide la cercanía, en cada instante de tiempo, a la trayectoria deseada $\overline{\mathbf{x}}(t)$. La función de los parámetros $\alpha_{j}, \beta_{j}>0$, es cuantificar la importancia relativa de las diferentes componentes del vector de estado respecto a cada uno de los criterios anteriores. Así, si se desea que una determinada componente $x_{i}$ del vector de estado cumpla la condición final, se tomará $\alpha_{i} \neq 0$, mientras que si no estamos interesados en que el resto de componentes cumplan la condición final tomaremos $\alpha_{j}=0 \forall j \neq i$. Del mismo modo, si se concede más importancia al seguimiento de la componente $i$ de la trayectoria que al de la componente $k$, se tomará $\beta_{i}>\beta_{k}$.

\subsection{Existencia de solución del problema de control óptimo}

Considérese un cierto estado inicial $\mathbf{x}^{0} \in \Omega$ y sea $t_{f}=t_{f}\left(\mathbf{x}^{0}\right)$ el tiempo maximal, según proporciona el Teorema 4.4, para el cual el sistema (4.5) está bien definido. El propósito de esta sección es probar el siguiente resultado de existencia: 
Teorema 5.1. Sean $\mathbf{x}^{0} \in \Omega$ y $0<t_{f}=t_{f}\left(\mathbf{x}^{0}\right)<+\infty$ en la forma definida con anterioridad. Existe, al menos, una solución de (9.4).

Demostración. La demostración se deriva del clásico teorema de Filippov de existencia de solución para problemas de control óptimo de Bolza (véase [Ces83, Th. 9.3.i, p. 314]). Veamos que se cumplen las condiciones suficientes para la existencia de solución. Debido a las restricciones sobre la variable de estado, el conjunto $\mathcal{A}=\left[0, t_{f}\right] \times \Omega$ es compacto. De igual modo, puesto que el conjunto de las restricciones sobre el control $\mathcal{K}=[0,1]^{2 N}$ es compacto, el conjunto $\mathcal{A} \times \mathcal{K}$ es también compacto. Por su parte, la función $\Phi: \mathcal{A} \times \mathcal{A} \rightarrow \mathbb{R}$ dada por (5.1) así como las funciones $F$ y $\mathbf{g}$, ambas definidas en $\mathcal{A} \times \mathcal{K}$, son continuas.

Gracias al Teorema 4.4, el conjunto de soluciones admisibles de (9.4) es no vacío. Finalmente, debemos comprobar que para cada $(t, \mathbf{x}) \in \mathcal{A}$ el campo de orientadores

$$
Q(t, \mathbf{x})=\left\{\left(z^{0} ; \mathbf{z}\right) \in \mathbb{R}^{1+3 N+12}: z^{0} \geq F(t, \mathbf{x}), \mathbf{z}=\mathbf{g}(t, \mathbf{x}, \mathbf{u}), \text { con } \mathbf{u} \in \mathcal{K}\right\}
$$

es convexo. Esta convexidad se deriva de forma inmediata del hecho de que la variable de control $\mathbf{u}$ aparece en forma lineal en la ley de estado y el conjunto $\mathcal{K}$ es convexo.

\subsubsection{Soplado y ventilación de tanques y superficies de control actuando de forma conjunta}

Si bien nuestro principal objetivo es estudiar los procesos de soplado y ventilación de tanques de lastre como un posible mecanismo de control alternativo, en ciertas situaciones será interesante considerar el uso conjunto de estos procesos y los medios tradicionales de control, como timones y hélice.

La existencia de solución para el problema de control óptimo empleando timones o timones y hélice fue probada en [PT10] y [GOP11]. En caso de incorporar el uso de los tanques de lastre, sin embargo, resulta difícil extender estos resultados debido a la dependencia respecto a la variable de estado de la matriz de masas $\mathbf{A}(\mathbf{x}(t))$. Si bien esto impide obtener de forma inmediata resultados de existencia como los citados ([GOP11, PT10]) o el Teorema 5.1, desde el punto de vista práctico pueden hacerse varias consideraciones:

a) Puesto que el problema tiene solución empleando timones y hélice y tiene también solución empleando el soplado y ventilación de los tanques, considerar las dos familias de controles de forma conjunta, es decir, aumentar el número de variables de control, no debería perjudicar la capacidad del algoritmo numérico de converger a una solución, incluso aunque desde el punto de vista matemático no pueda probarse la existencia de un mínimo (el coste podría, por ejemplo, disminuir indefinidamente). De hecho, los mínimos que se obtendrían empleando únicamente los timones o únicamente el soplado/ventilación serían, desde el punto de vista numérico, soluciones particulares del problema de considerar ambas familias de controles de forma conjunta. 
b) Es posible evitar el carácter no lineal de timones y hélice como variables de control mediante un cambio de variable que permita considerar los ángulos de giro de los timones y la velocidad de giro de la hélice como nuevas variables de estado. De esta forma, pueden tomarse como nuevas variables de control sus derivadas, apareciendo nuevas ecuaciones de la forma

$$
\dot{\delta}_{r}=u_{\delta_{r}}
$$

Puesto que los controles aparecen ahora en estas ecuaciones en forma lineal, el Teorema 5.1 es suficiente para probar la existencia de solución.

Nótese, sin embargo, que pese a que este problema es muy similar al original, ambos problemas no son equivalentes, ya que ahora las variables de control relativas a timones y hélice son sus velocidades de cambio y no sus valores instantáneos. Esto implica, por ejemplo, que han de fijarse condiciones iniciales para las ahora nuevas variables de estado.

\subsection{Resolución numérica}

Existen diversos métodos que pueden emplearse para la resolución de (9.4). Dada la complejidad de la ley de estado y el elevado número de variables del problema, es razonable emplear un método de descenso tipo gradiente (una descripción de éste y otros métodos clásicos puede encontrarse en [Kir04]). Este método se basa en el hecho bien conocido de que el gradiente de una función en un punto marca la dirección en la que ésta crece más rápidamente; la dirección opuesta al gradiente, por tanto, marca la dirección en la que decrece de forma más rápida (de ahí el nombre steepest descent con el que este método es generalmente conocido en la literatura). Si llamamos $\nabla J(\mathbf{u})$ al gradiente de la función coste, el procedimiento propuesto consiste en partir de un control inicial $\mathbf{u}^{0}$ y proceder de forma iterativa avanzando en dirección $-\nabla J(\mathbf{u})$ hasta satisfacer un cierto criterio de convergencia. La clave, por tanto, es el cálculo del gradiente $\nabla J(\mathbf{u})$. Puede emplearse, para ello, el método del estado adjunto (pasos 2.12.3 abajo). Véase [Cul94, Kir04] para mayor detalle acerca de este método. En resumen, el algoritmo propuesto puede sintetizarse como:

1. Inicialización del vector de control $\mathbf{u}^{0}$.

2. Para $k \geq 0$, proceder de forma iterativa hasta satisfacer un cierto criterio de convergencia, como por ejemplo $\left|J\left(\mathbf{u}^{k+1}\right)-J\left(\mathbf{u}^{k}\right)\right| \leq \varepsilon\left|J\left(\mathbf{u}^{0}\right)\right|$, con $\varepsilon>0$ una tolerancia admisible.

2.1 Dado el control $\mathbf{u}^{k}$, resolver la ley de estado

$$
\left\{\begin{array}{l}
\mathbf{A}(\mathbf{x}(t)) \dot{\mathbf{x}}(t)=\mathbf{f}\left(t, \mathbf{x}(t), \mathbf{u}^{k}(t)\right) \\
\mathbf{x}(0)=\mathbf{x}^{k}(0)
\end{array}\right.
$$

para obtener el vector de estado $\mathbf{x}^{k+1}(t)$. 
2.2 Con $\left(\mathbf{u}^{k}(t), \mathbf{x}^{k+1}(t)\right)$, resolver hacia atrás en el tiempo la ecuación del estado adjunto $\mathbf{p}(t)$

$$
\left\{\begin{array}{l}
\mathbf{A}\left(\mathbf{x}^{k+1}(t)\right)^{T} \dot{\mathbf{p}}(t)=-\nabla_{\mathbf{x}} F\left(t, \mathbf{x}^{k+1}(t), \mathbf{u}^{k}(t)\right)-\left[\nabla_{\mathbf{x}} \mathbf{f}\left(t, \mathbf{x}^{k+1}(t), \mathbf{u}^{k}(t)\right)\right]^{T} \mathbf{p}(t) \\
\mathbf{A}\left(\mathbf{x}^{k+1}\left(t_{f}\right)\right)^{T} \mathbf{p}\left(t_{f}\right)=\nabla_{\mathbf{x}} \Phi\left(\mathbf{x}^{k+1}\left(t_{f}\right), \mathbf{x}^{t_{f}}\right)
\end{array}\right.
$$

donde $\nabla_{\mathbf{x}}$ es el gradiente respecto a la variable de estado $\mathbf{x}$. Se obtiene así $\mathbf{p}^{k+1}(t)$.

$2.3 \mathrm{El}$ gradiente de la función coste puede obtenerse como

$$
\nabla J\left(\mathbf{u}^{k}\right)=\nabla_{\mathbf{u}} F\left(t, \mathbf{x}^{k+1}(t), \mathbf{u}^{k}(t)\right)+\left[\nabla_{\mathbf{u}} \mathbf{f}\left(t, \mathbf{x}^{k+1}(t), \mathbf{u}^{k}(t)\right)\right]^{T} \mathbf{p}^{k+1}(t)
$$

donde $\nabla_{\mathbf{u}}$ denota el gradiente respecto a la variable de control.

$2.4 \mathrm{El}$ nuevo vector de control es

$$
\mathbf{v}^{k+1}=\mathbf{u}^{k}-\lambda \nabla J\left(\mathbf{u}^{k}\right)
$$

siendo $\lambda>0$ un tamaño de paso constante.

2.5 Puesto que $\mathbf{v}^{k+1}$ puede no ser admisible, calculamos su proyección ortogonal en el conjunto de admisibilidad $\mathcal{K}$, el rectángulo unidad en $\mathbb{R}^{8}$, es decir,

$$
\mathbf{u}^{k+1}=\mathcal{P}_{K}\left(\mathbf{v}^{k+1}\right)
$$

donde

$$
\mathbf{u}_{j}^{k+1}=\operatorname{mín}\left(\operatorname{máx}\left(0, \mathbf{v}_{j}^{k+1}\right), 1\right) \text {. }
$$

En capítulos posteriores emplearemos este algoritmo de control para el estudio de maniobras de ascenso de emergencia (Capítulo 8, secciones 8.1.1 y 8.2) y el control en lazo abierto del hovering (Capítulo 9, Sección 9.3.1). 



\section{Algoritmo feedback para el seguimiento de trayectorias óptimas}

Como vimos en el capítulo anterior, el Cálculo de Variaciones y el Principio de Pontryagin proporcionan las herramientas necesarias para resolver un amplio espectro de problemas de control óptimo en los que los controles óptimos son función únicamente del tiempo, es decir, proporcionan la solución a un problema de control en lazo abierto. Desde el punto de vista práctico, esto es útil para estudiar el comportamiento dinámico de un sistema, el cálculo de trayectorias óptimas o el diseño preliminar de sistemas de control. En la mayoría de aplicaciones finales, sin embargo, el diseño de sistemas de control requiere el uso de algoritmos de control en lazo cerrado o feedback que permitan corregir las desviaciones del comportamiento esperado producidas, por ejemplo, por errores en el modelado del sistema (como puede ser en nuestro caso la estimación de los coeficientes hidrodinámicos) o la acción de agentes externos (como las perturbaciones tratadas en el Capítulo 2).

El propósito del presente capítulo es proponer un algoritmo feedback para el cálculo de controles óptimos que puede ser empleado para el seguimiento de trayectorias generadas mediante la metodología expuesta en el capítulo anterior -como veremos, el método es más general y no está estrictamente limitado al seguimiento de trayectorias--

En la Sección 6.1 formularemos el problema que estamos interesados en resolver, mientras que en la Sección 6.2 se aborda el algoritmo propuesto para su resolución. Con el fin de ilustrar el uso de este algoritmo, en la Sección 6.3 se resuelve por medio de éste un sencillo ejemplo académico para el que puede encontrarse solución explícita. Por último, la Sección 6.4 
presenta una posible extensión del esquema propuesto que permite mejorar la precisión de los resultados obtenidos en determinados problemas.

\subsection{Formulación del problema}

Consideremos el siguiente problema de control óptimo: dado un tiempo final $t_{f}$, encontrar el control $u:\left[0, t_{f}\right] \rightarrow K \subset \mathbb{R}^{m}$ solución de

$$
(\mathrm{OCP}) \begin{cases}\text { Minimizar en } u: & J(u)=\int_{t_{0}}^{t_{f}} F(t, x(t)) d t \\ \text { sujeto a } & \\ & x^{\prime}(t)=f(t, x(t), u(t)) \\ & x\left(t_{0}\right)=x^{0} \\ & u(t) \in K\end{cases}
$$

siendo $x^{0}$ la condición inicial y $K$ el conjunto de controles admisibles.

Como vemos, restringimos nuestro estudio al caso en que el integrando presente en la función coste no depende de la variable de control. Esto incluye, aunque no de forma exclusiva (la Sección 6.3 muestra un ejemplo en el que no existe una trayectoria a seguir), el problema conocido como trajectory tracking, en el que el sistema de control busca seguir tan fielmente como sea posible una trayectoria deseada conocida de antemano.

Hemos visto con anterioridad como, bajo ciertas condiciones, podemos obtener controles óptimos en la forma $u=u(t)$. En el presente caso, sin embargo, nuestro objetivo es obtener controles en lazo cerrado, de modo que puedan corregirse errores en el modelado del sistema o los efectos de perturbaciones externas. Construiremos una malla temporal $t_{0}<t_{1}<t_{2}<$ $\cdots<t_{N+1}=t_{f}$, en la que, para cada instante de tiempo discreto $t_{j}$, podemos medir el valor real de la variable de estado $x\left(t_{j}\right)=x^{j}$. Partiendo de estas mediciones, buscaremos el control $u^{j}=u^{j}\left(x^{j}\right)$, constante durante el intervalo $\left[t_{j}, t_{j+1}[\right.$, que hace que la evolución de la variable de estado durante este subintervalo sea óptima. En términos matemáticos, tenemos la serie de problemas

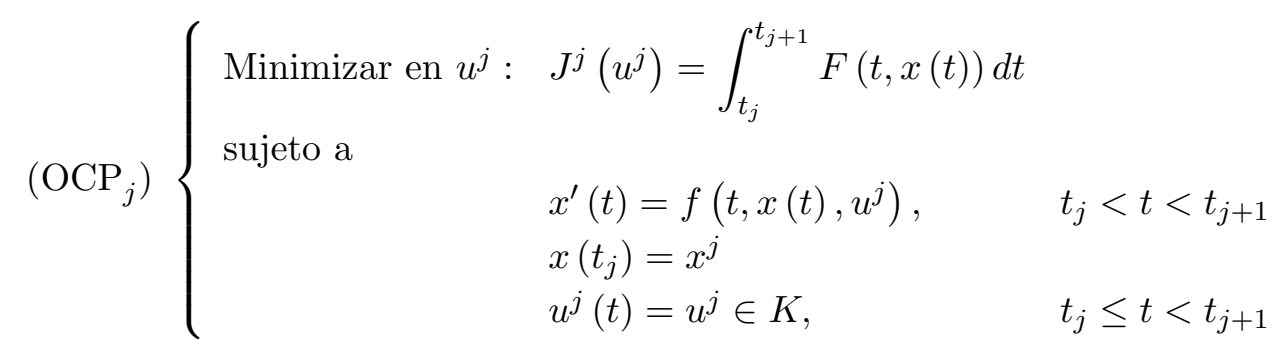




\subsection{Esquema numérico}

Como es bien sabido, bajo ciertas condiciones (los detalles pueden encontrarse, por ejemplo, en [Ped03, Prop. 3.1]), (OCP) es equivalente al problema variacional

$$
(\mathrm{VP}) \begin{cases}\text { Minimizar en } x: & \bar{J}(x)=\int_{t_{0}}^{t_{f}} \phi\left(t, x(t), x^{\prime}(t)\right) d t \\ \text { sujeto a } & x\left(t_{0}\right)=x^{0}\end{cases}
$$

donde la densidad $\phi$ viene dada por

$$
\phi(t, x, \xi)=\min _{u \in K, \xi=f(t, x, u)} F(t, x) .
$$

Por simplicidad, supondremos que la malla temporal $t_{0}<t_{1}<t_{2}<\cdots<t_{N+1}=t_{f}$ es de la forma

$$
t_{j+1}=t_{j}+h, \quad h=\left(t_{f}-t_{0}\right) / N, \quad N \in \mathbb{N} .
$$

En esta malla, (VP) adopta la forma

$$
\left(\mathrm{VP}_{j}\right) \begin{cases}\text { Minimizar en } x: & \bar{J}^{j}(x)=\int_{t_{j}}^{t_{j+1}} \phi\left(t, x(t), x^{\prime}(t)\right) d t \\ \text { sujeto a } & x\left(t_{j}\right)=x^{j}\end{cases}
$$

donde $\phi$ está dada por (6.1) y las condiciones iniciales $x^{j}$ son conocidas.

Puesto que en $\left(\mathrm{VP}_{j}\right)$ no se ha impuesto condición final podemos calcular la pendiente óptima $\xi^{j}$ en cada nodo $t_{j}$. Para ello, aproximamos el coste $\bar{J}^{j}(x)$ en la forma

$$
\bar{J}^{j}(x)=\int_{t_{j}}^{t_{j+1}} \phi\left(t, x(t), x^{\prime}(t)\right) d t \approx h \phi\left(t_{j}+h, x^{j}+h \xi^{j}, \xi^{j}\right),
$$

donde se ha tomado la aproximación de primer orden $x\left(t_{j+1}\right) \approx x^{j}+h \xi^{j}$, con $x\left(t_{j}\right)=x^{j} \mathrm{y}$ $x^{\prime}\left(t_{j}\right)=\xi^{j}$.

Por tanto, una aproximación $\xi_{h}^{j}$ de la pendiente óptima $\xi^{j}$ en $t_{j}$ es solución del problema de programación no lineal

$$
\min _{\xi} \phi\left(t_{j}+h, x^{j}+h \xi, \xi\right) .
$$

Sustituyendo este último problema en (6.1) obtenemos el doble problema de optimización

$$
\min _{\xi} \min _{u \in K, \xi\left(t_{j}+h, x^{j}+h \xi, u\right)} F\left(t_{j}+h, x^{j}+h \xi\right) .
$$

Finalmente, para $h$ lo suficientemente pequeño, la aproximación buscada de la pendiente óptima $\xi_{h}^{j}$ y del control óptimo en forma feedback $u_{h}^{j}$ puede obtenerse como solución del 
siguiente problema de programación matemática no lineal con restricciones:

$$
\left(\mathrm{NPP}_{h}^{j}\right) \begin{cases}\text { Minimizar en }(u, \xi): & F\left(t_{j}+h, x^{j}+h \xi\right) \\ \text { subjeto a } & \\ & u \in K \\ & \xi=f\left(t_{j}+h, x^{j}+h \xi, u\right)\end{cases}
$$

En conclusión, el esquema numérico propuesto se reduce a resolver en cada nodo temporal el problema $\left(\mathrm{NPP}_{h}^{j}\right)$ conocido el valor de variable de estado $x^{j}$.

\subsection{Un ejemplo académico}

Con el fin de obtener una mejor comprensión del funcionamiento del algoritmo propuesto así como de algunas de sus principales propiedades, se presenta en esta sección un sencillo ejemplo académico para el que puede obtenerse una solución explícita.

Consideremos el problema de control óptimo

$$
\left(\mathrm{OCP}^{1}\right)\left\{\begin{array}{lll}
\text { Minimizar en } u: & J(u)=\int_{0}^{1} x^{2}(t) d t & \\
\text { sujeto a } & & \\
& x^{\prime}(t)=-x(t)+u(t), & 0<t<1 \\
& x(0)=1 & \\
& |u(t)| \leq 1, & 0 \leq t \leq 1 .
\end{array}\right.
$$

La reformulación variacional de $\left(\mathrm{OCP}^{1}\right)$ toma la forma

$$
\left(\mathrm{VP}^{1}\right) \begin{cases}\text { Minimizar en } x: & \bar{J}(x)=\int_{0}^{1} \phi\left(t, x(t), x^{\prime}(t)\right) d t \\ \text { sujeto a } & x(0)=1\end{cases}
$$

donde

$$
\phi(t, x, \xi)= \begin{cases}\min _{u \in K} x^{2}(t) & \text { si } \xi=-x(t)+u \\ +\infty & \text { en caso contrario. }\end{cases}
$$

Es sencillo ver que la forma óptima de proceder es descender con la mayor pendiente posible, $u=-1$ y por tanto $\xi=-x-1$, hasta que $x\left(t^{*}\right)=0$ y a partir de ese momento y hasta el final $(t=1)$, tomar $u=0$. La Figura 6.1 muestra una representación geométrica del problema en el plano $x-\xi$.

Así pues, el control óptimo y el estado asociado pueden obtenerse de forma explícita como

$$
u_{\text {opt }}(t)=\left\{\begin{array}{ll}
-1, & 0 \leq t<\ln 2 \\
0, & \ln 2 \leq t \leq 1
\end{array} \quad \mathrm{y} \quad x_{\text {opt }}(t)= \begin{cases}2 e^{-t}-1, & 0 \leq t<\ln 2 \\
0, & \ln 2 \leq t \leq 1\end{cases}\right.
$$




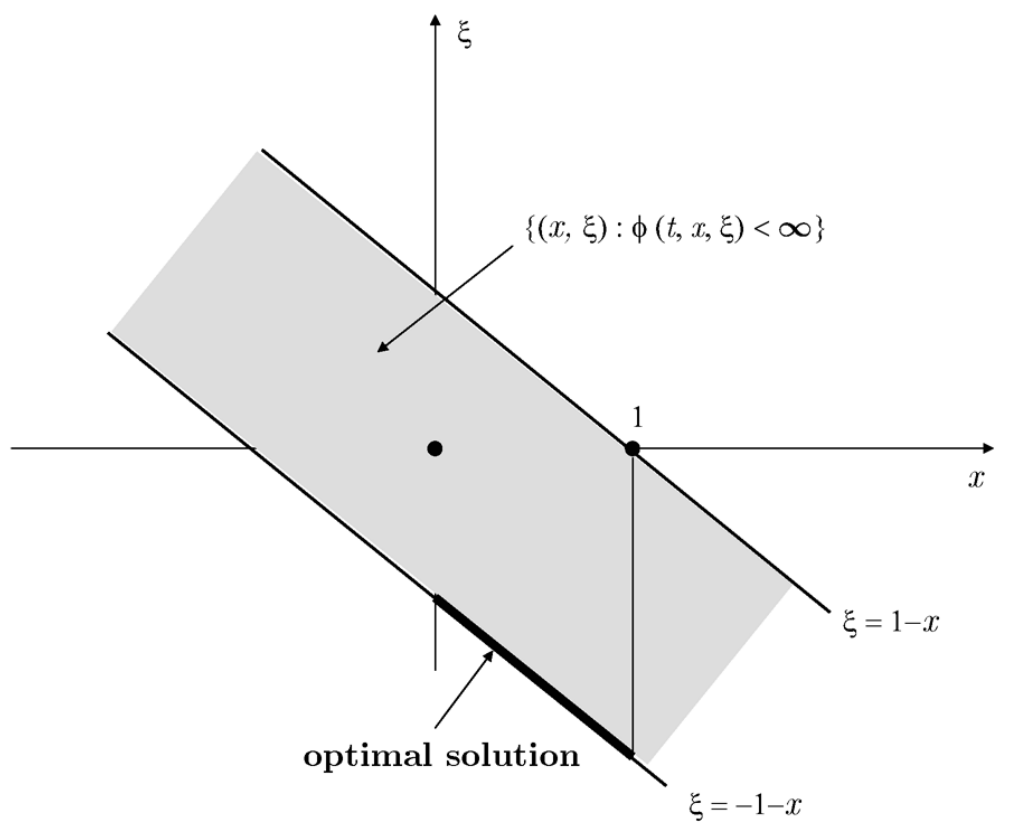

Figura 6.1: Representación del conjunto en el que $\phi$ es finito y la solución óptima de $\left(\mathrm{VP}^{1}\right)$.

Mientras que el coste óptimo será

$$
\bar{J}\left(x_{\text {opt }}\right)=\int_{0}^{\ln 2}\left(2 e^{-t}-1\right)^{2} d t=-0,5+\ln 2 \approx 0,1931 .
$$

En caso de trabajar sobre una determinada malla temporal como se describió en la sección anterior, tendremos la serie de problemas de control óptimo en forma feedback

$$
\left(\mathrm{OCP}_{j}^{1}\right) \begin{cases}\text { Minimizar en } u_{j}: & J^{j}\left(u^{j}\right)=\int_{t_{j}}^{t_{j+1}} x^{2}(t) d t \\ \text { sujeto a } & \\ & x^{\prime}(t)=-x(t)+u^{j}(t), \quad t_{j}<t<t_{j+1} \\ & x\left(t_{j}\right)=x^{j} \\ & u^{j}(t)=u^{j} \in K=[-1,1,] \quad t_{j} \leq t<t_{j+1} .\end{cases}
$$

La aproximación $u_{h}^{j}$ de la solución $\left(\mathrm{OCP}_{j}^{1}\right)$ puede obtenerse resolviendo el problema de programación matemática no lineal con restricciones

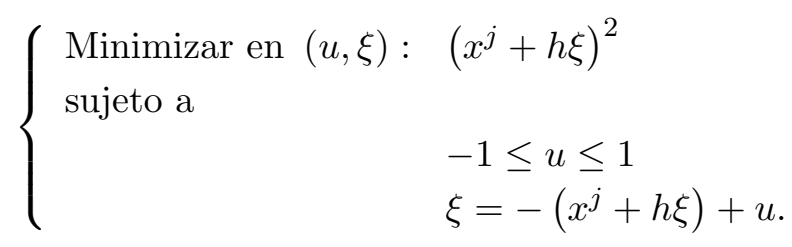


La solución de este problema puede obtenerse explícitamente resolviendo las condiciones necesarias de optimalidad de Kuhn-Tucker. En efecto,

$$
\left(u_{h}^{j}, \xi_{h}^{j}\right)= \begin{cases}\left(+1, \frac{1-x^{j}}{1+h}\right) & \text { si } x^{j}<-h \\ \left(-x^{j} / h,-x^{j} / h\right) & \text { si }-h \leq x^{j} \leq h \\ \left(-1,-\frac{1+x^{j}}{1+h}\right) & \text { si } x^{j}>h .\end{cases}
$$

La Tabla 6.1 muestra la evolución del valor de la función coste $J_{h}=\int_{0}^{1}\left(x_{h}(t)\right)^{2} d t$ con respecto al periodo de muestreo $h$ y para condiciones iniciales $x^{j}=x_{\text {opt }}\left(t_{j}\right)$. En la Figura 6.2 se muestran los controles y estados óptimos para $h=0,1$ y $h=0,001$.

\begin{tabular}{llll}
\hline \hline$h$ & 0,1 & 0,01 & 0,001 \\
\hline \hline$J_{h}$ & 0,1932 & 0,1931 & 0,1931 \\
\hline \hline
\end{tabular}

Tabla 6.1: Valores del coste óptimo $J_{h}$ para diferentes valores de $h$.
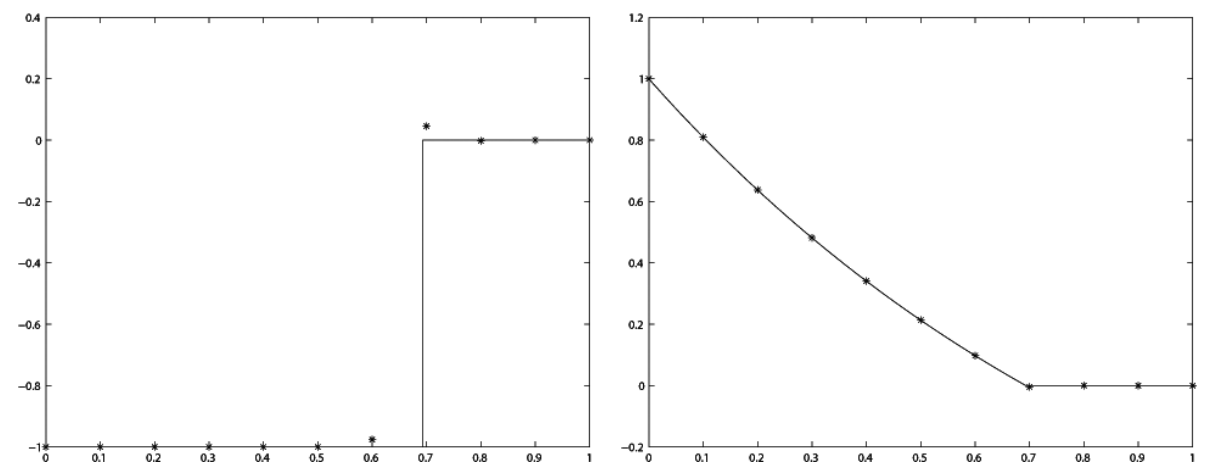

Figura 6.2: Control óptimo feedback $u(t), 0 \leq t \leq 1$ (izquierda) y estado asociado $x(t)$,

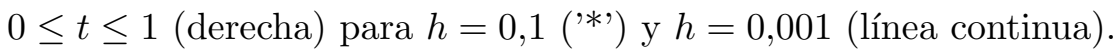

Puede apreciarse en estos resultados cómo la solución discreta proporcionada por (6.4) converge a la solución exacta (6.2). De hecho, esta convergencia puede ser demostrada analíticamente. Es sencillo ver que

$$
x^{j} \leq h \quad \text { si y sólo si } t_{j}=j h \geq \ln \frac{2}{1+h} .
$$

Así pues, para un $h=1 / N$ fijo existe un $j_{0}$ tal que

$$
\ln \frac{2}{1+h} \leq t_{j_{0}}<\ln 2<t_{j_{0}+1} .
$$

Por tanto

$$
u_{h}(t)= \begin{cases}-1, & 0 \leq t<t_{j_{0}} \\ -x^{j} / h, & t_{j_{0}} \leq t<t_{j_{0}+1} \\ 0, & t>t_{j_{0}+1}\end{cases}
$$


Como consecuencia,

$$
\left\|u_{h}-u_{o p t}\right\|_{L^{p}(0,1)}^{p}=\int_{t_{j_{0}}}^{\ln 2}\left|1-x^{j} / h\right|^{p} d t+\int_{\ln 2}^{t_{j_{0}+1}}\left|-x^{j} / h\right|^{p} d t \leq\left(1+2^{p}\right) h .
$$

En particular,

$$
u_{h} \rightarrow u_{o p t} \quad \text { c.t.p. } t \in[0,1]
$$

Un cálculo similar muestra que $J\left(u_{h}-u_{\text {opt }}\right) \rightarrow 0$ conforme $h \rightarrow 0$, lo que implica que $\left\{u_{h}\right\}$ es una sucesión minimizante para $\left(\mathrm{OCP}^{1}\right)$.

Con el fin de poner a prueba la robustez del algoritmo propuesto frente a errores de medición, las mediciones de $x_{h}(t)$, para $h=0,01$ han sido afectadas por un ruido blanco siguiendo una distribución gaussiana con diferentes desviaciones típicas $\sigma$. La Figura 6.3 muestra los resultados de simulación obtenidos para la variable de estado perturbada y no perturbada así como el control no perturbado y el obtenido mediante el algoritmo propuesto tomando como entrada la variable de estado perturbada con $\sigma=0,1, \sigma=0,001$ y $\sigma=0,0001$.

Puesto que la perturbación considerada es de naturaleza aleatoria, es necesario repetir este experimento un cierto número de veces con el fin de que las posibles conclusiones puedan ser apoyadas en criterios probabilísticos. La Figura 6.4 muestra los resultados tras 100 realizaciones de este mismo experimento. En el eje horizontal se representa el error en el estado perturbado $x_{h}^{\sigma}(t)$ respecto al no perturbado, $x_{h}(t)$, mientras que en el eje vertical se muestra el error en los controles $u_{h}(t)$ y $u_{h}^{\sigma}(t)$-ambos obtenidos mediante el algoritmo propuestopara $\sigma=0,1$ y $\sigma=0,0001$ en las normas $L^{2}$ y $L^{\infty}$. Cada punto en estas gráficas representa el resultado correspondiente a cada uno de los experimentos (sobre un total de 100 realizaciones). Como puede verse (Figura 6.4, arriba a la izquierda), una perturbación en la variable de estado del orden de 0,3 unidades en la norma $L^{\infty}$ produce un error máximo en la variable de control de alrededor de 2 unidades en la norma $L^{\infty}$. Del mismo modo, un error en la variable de estado de alrededor de $10^{-4}$ en la norma $L^{\infty}$ produce un error en la variable de control del orden de $10^{-2}$ unidades en esta misma norma (Figura 6.4, arriba a la derecha). En la sección inferior se muestran estos mismos resultados para la norma $L^{2}$.

Finalmente, podemos comparar los resultados proporcionados por el algoritmo propuesto con los obtenidos aplicando, en cada nodo de la malla temporal, un sencillo método de transcripción. Para ello, aproximaremos la ley de estado $x^{\prime}(t)=-x(t)+u(t)$ en el subintervalo de tiempo $\left[t_{j}, t_{j+1}[\right.$ mediante un desarrollo en serie de Taylor de primer orden:

$$
\frac{x^{j+1}-x^{j}}{h}=-x^{j}+u^{j}
$$

De forma similar, aproximaremos el valor de la integral en la función coste a lo largo de este subintervalo de tiempo por su valor final. Estas dos aproximaciones dan lugar a la familia de 

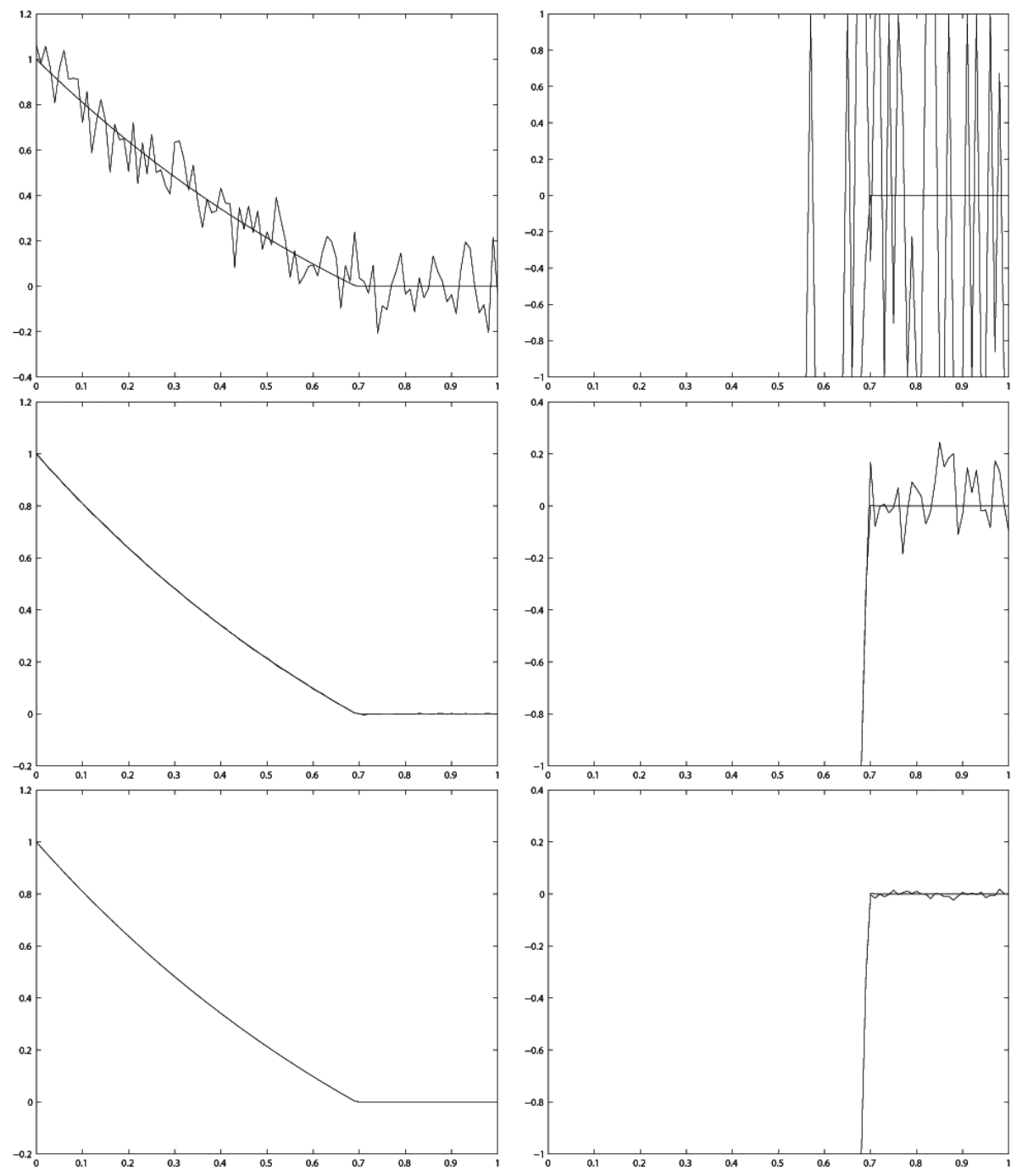

Figura 6.3: La columna izquierda muestra la variable de estado $x(t), 0 \leq t \leq 1$ no perturbada y afectada por ruido blanco aditivo con $\sigma=0,1$ (arriba), $\sigma=0,001$ (medio) y $\sigma=0,0001$ (abajo). La columna derecha muestra, en el mismo orden, la correspondiente variable de control $u(t), 0 \leq t \leq 1$.

problemas

$$
\begin{cases}\text { Minimizar en }\left(u^{j}, x^{j+1}\right): & \left(x^{j+1}\right)^{2} \\ \text { sujeto a } & \frac{x^{j+1}-x^{j}}{h}=-x^{j}+u^{j} \\ & u^{j} \in[-1,1] .\end{cases}
$$

Este problema de programación matemática no lineal puede ser fácilmente resuelto. En con- 

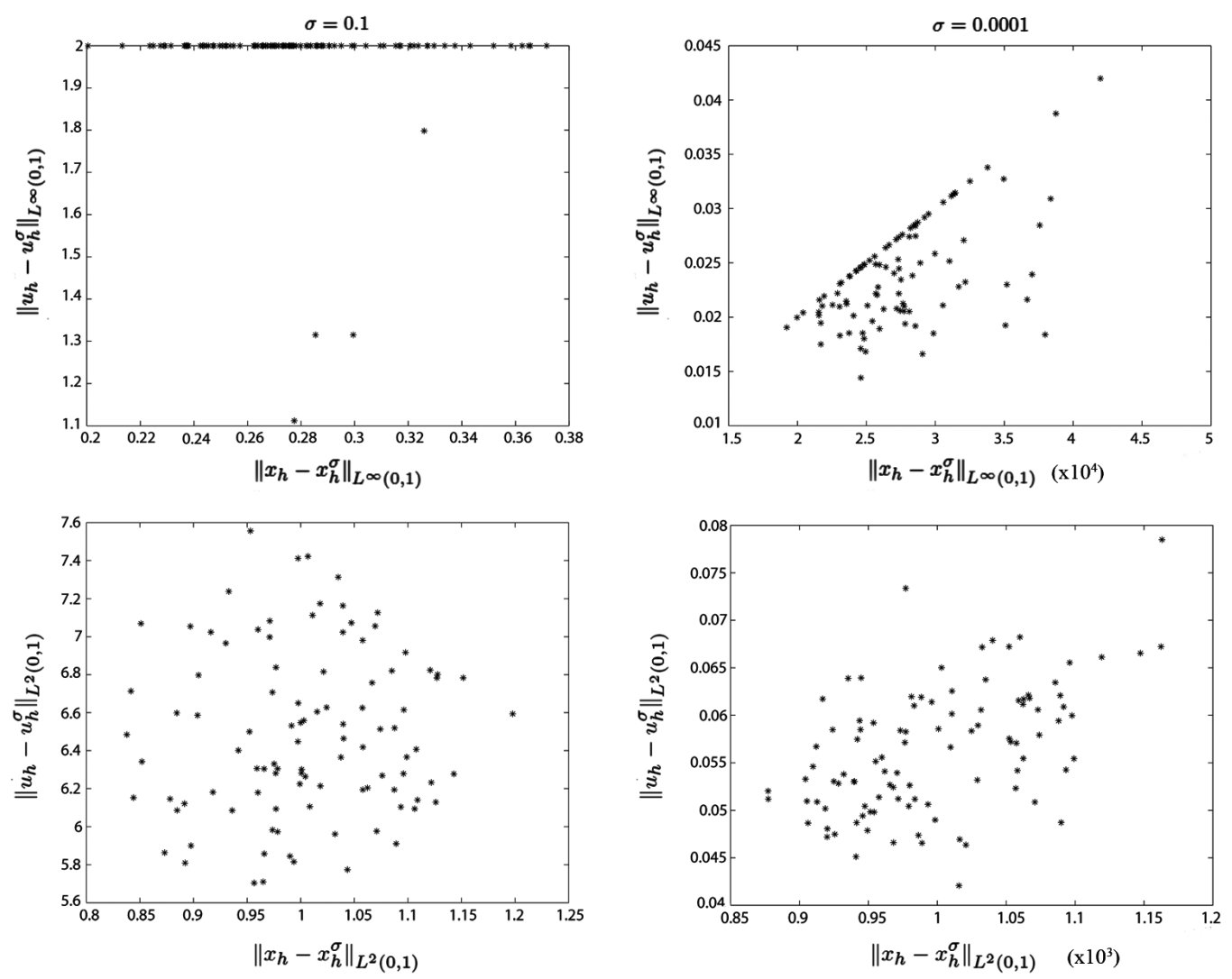

Figura 6.4: Resultados para el error inducido en la variable de control por una perturbación aleatoria de la variable de estado. $\left\|x_{h}-x_{h}^{\sigma}\right\|_{L^{\infty}(0,1)} \quad$ vs. $\quad\left\|u_{h}-u_{h}^{\sigma}\right\|_{L^{\infty}(0,1)}$ para $\sigma=0,1$ (arriba a la izquierda) y $\sigma=0,0001$ (arriba a la derecha). $\left\|x_{h}-x_{h}^{\sigma}\right\|_{L^{2}(0,1)} \quad$ vs. $\quad\left\|u_{h}-u_{h}^{\sigma}\right\|_{L^{2}(0,1)}$ para $\sigma=0,1$ (abajo a la derecha) y $\sigma=0,0001$ (abajo a la derecha).

creto, obtenemos

$$
u^{j}= \begin{cases}-\frac{1-h}{h} x^{j} & \text { si }-\frac{h}{1-h} \leq x^{j} \leq \frac{h}{1-h} \\ -1 & \text { en caso contrario }\end{cases}
$$

y $x^{j+1}=(1-h) x^{j}+h u^{j}$.

Esta solución es diferente de (6.4), aunque los resultados numéricos son muy similares a los obtenidos mediante el algoritmo propuesto. Esto es debido, muy probablemente, a que en el ejemplo considerado el término de la derecha de la ley de estado es lineal, por lo que la solución es suave. Por tanto, un desarrollo en serie de Taylor de primer orden proporciona una buena aproximación de $x^{\prime}(t)$ en $\left[t_{j}, t_{j+1}\right.$ [ para un $h$ suficientemente pequeño. Nótese que, pese a que $x^{j+1}$ se supone un dato medido, aparece en el problema (6.5) como una variable artificial. De este modo, al pasar del punto $t_{j}$ de la malla de tiempos al nodo siguiente, $t_{j+1}$, el valor de $x^{j+1}$ debe ser actualizado con los valores medidos. Es interesante destacar, 
también, que la diferencia entre los problemas (6.3) y (6.5) no se limita únicamente a las incógnitas $\left((u, \xi)\right.$ en $(6.3)$ y $\left(u, x^{j+1}\right)$ en $\left.(6.5)\right)$, sino también al modo en que éstas aparecen en las restricciones de igualdad. En el primer caso, $\xi$ aparece (en general) en forma implícita, mientras que en el segundo $x^{j+1}$ lo hace en forma explícita.

En capítulos posteriores veremos que, para problemas complejos como el de la maniobrabilidad de vehículos submarinos, el algoritmo propuesto proporciona resultados algo más precisos que los obtenidos mediante el método de transcripción y, lo que es más importante, la convergencia en cada uno de los subintervalos es notablemente más rápida, reduciendo en gran medida el tiempo de cálculo necesario.

\subsection{Posible extensión del método}

Presentamos en esta sección una posible extensión del método propuesto que permite, en determinados casos, mejorar los resultados obtenidos a costa de aumentar ligeramente la complejidad de los cálculos. Introduciremos, en primer lugar, un pequeño cambio en la notación empleada en secciones precedentes.

Sea el problema de programación matemática no lineal $\left(\mathrm{NPP}_{h}^{j}\right)$ :

$$
\left(\mathrm{NPP}_{h}^{j}\right) \begin{cases}\text { Minimizar en }(u, \xi): & F\left(t_{j}+h, x^{j}+h \xi\right) \\ \text { sujeto a } & \\ & u \in K \\ & \xi=f\left(t_{j}+h, x^{j}+h \xi, u\right)\end{cases}
$$

Puesto que $\xi$ es constante en $\left[t_{j}, t_{j}+h[\right.$, puede escribirse

$$
x_{j+1}=x_{j}+h \xi
$$

donde este $x_{j+1}$ es el valor predicho para el instante $t_{j+1}$ calculado en $t_{j}$. Para distinguirlos de los datos medidos, denotemos los valores predichos por $\bar{x}\left(t_{j}+h\right)$. De este modo $\left(\mathrm{NPP}_{h}^{j}\right)$ puede reescribirse como

$$
\left(\overline{\mathrm{NLPP}}_{h}^{j}\right) \begin{cases}\text { Minimizar en }\left(u, \bar{x}\left(t_{j}+h\right)\right): & F\left(t_{j}+h, \bar{x}\left(t_{j}+h\right)\right) \\ \text { sujeto a } & \\ & u \in K \\ & \bar{x}\left(t_{j}+h\right)=x_{j}+h f\left(t_{j}+h, \bar{x}\left(t_{j}+h\right), u\right)\end{cases}
$$

Consideremos de nuevo la serie de problemas de control óptimo en la forma 


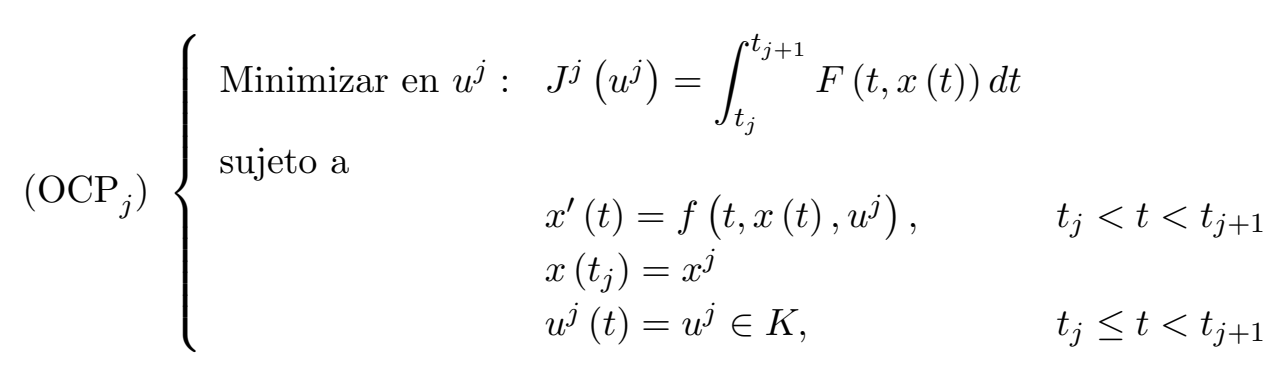

La extensión propuesta para el algoritmo se basa en las ideas del control predictivo basado en modelo (Model Predictive Control, MPC). Puede encontrarse una completa introducción al MPC en [CB04] y mayor detalle en [CB07]. La idea fundamental de la modificación propuesta es tomar un cierto horizonte temporal $T>h$, de forma que, para cada $t_{j}$, en lugar de resolver el problema $\left(\mathrm{OCP}_{j}\right)$ para $\left[t_{j}, t_{j}+h\left[\right.\right.$ lo haremos para el intervalo $\left[t_{j}, t_{j}+T[\right.$.

Optimizando en un intervalo temporal mayor, buscamos obtener acciones de control suaves, evitando que el seguimiento actual de la referencia perjudique el seguimiento futuro. Consideremos, por ejemplo, un ejemplo extremo como el de la Figura 6.5. Buscamos seguir una trayectoria deseada $x_{d}$ y en el instante $t_{j}$ nos encontramos en el punto $x^{j}$, notablemente alejado de $x_{d}^{j}$. Si buscamos alcanzar la trayectoria en $t_{j+1}$ (línea roja), no sólo necesitaremos una acción de control probablemente muy brusca, sino que al llegar a $x_{d}^{j+1}$ la tendencia será rebasar la trayectoria buscada, necesitándose de nuevo una brusca acción de control en sentido contrario. En cambio, si consideramos un tiempo mayor, será posible aproximarnos a la trayectoria de forma suave (línea azul) y con menor acción de control.

De esta forma, en lugar de $\left(\mathrm{OCP}_{j}\right)$, resolveremos la serie de problemas

$$
\left(\mathrm{OCP}_{T}^{j}\right) \begin{cases}\text { Minimizar en } u & J^{j}(u)=\int_{t_{j}}^{t_{j}+T} F(t, x(t)) d t \\ \text { sujeto a } & \\ & x^{\prime}(t)=f(t, x(t), u) \\ & x\left(t_{j}\right)=x^{j} \\ & u(t) \in K\end{cases}
$$

donde, como vemos, no se exige, en general, que $u$ sea constante en todo el intervalo. Como veremos más adelante, puede exigirse que lo sea en todo el intervalo o bien en subintervalos más pequeños.

En lugar de aproximar el valor de la integral en $\left(\mathrm{OCP}_{T}^{j}\right)$ mediante el valor en la derecha, la aproximaremos mediante el valor en una serie de puntos equiespaciados. Sea $N_{J}$ el número de subintervalos en el que deseamos dividir el intervalo $\left[t_{j}, t_{j}+T\left[\right.\right.$, y $H=\frac{T}{N_{J}}$ la longitud de cada subintervalo. La integral en $\left(\mathrm{OCP}_{T}^{j}\right)$ puede aproximarse como: 


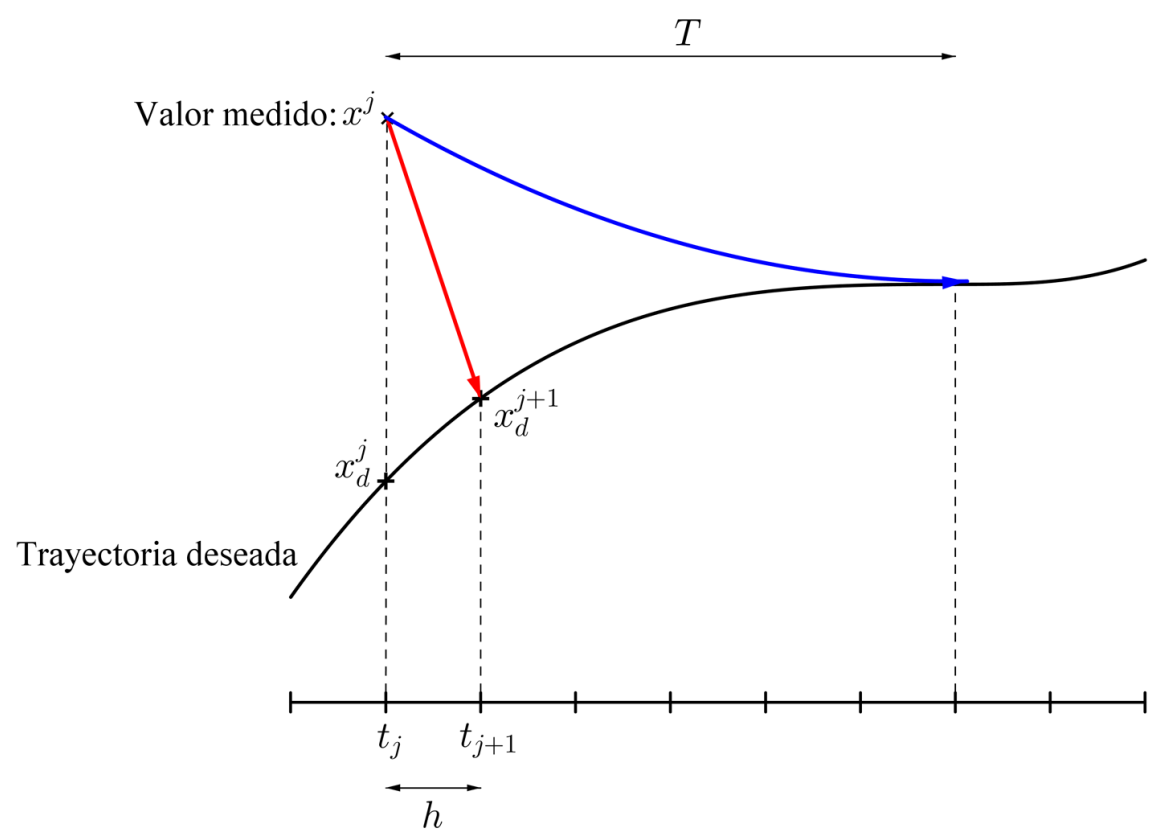

Figura 6.5: Elección de un horizonte $T>h$ para la optimización.

$$
J^{j}(u)=\int_{t_{j}}^{t_{j}+T} F(t, x(t)) d t \simeq H \sum_{i=1}^{N_{J}} F_{i}\left(t_{j}+i H, x\left(t_{j}+i H\right)\right)
$$

De esta forma, intentamos que la referencia se alcance, no sólo con precisión en el instante final, sino razonablemente a lo largo de un intervalo más amplio.

Llamemos ahora $N_{u} \leq N_{J}$ al número de intervalos de control considerados. Lo que esto quiere decir es que, tras $N_{u}$ subintervalos, el control se considera constante para los restantes $N_{J}-N_{u}$ subintervalos. De esta forma, si $N_{u}=1$ el control se considera constante en todo $\left[t_{j}, t_{j}+T\left[\right.\right.$, mientras que si $N_{u}=2$ se tomará un valor constante en $\left[t_{j}, t_{j}+H[\right.$, otro en $\left[t_{j}+H, t_{j}+2 H\left[\right.\right.$ y éste último se mantendrá para $\left[t_{j}+2 H, t_{j}+N_{J} H[\right.$, y así sucesivamente hasta el caso $N_{u}=N_{J}$ en el que habría un control distinto para cada subintervalo.

De esta forma, $\left(\mathrm{NPP}_{h}^{j}\right)$ es reemplazado por el siguiente problema de programación matemática 
no lineal

$$
\begin{aligned}
& \begin{cases}\text { Minimizar } & \sum_{i=1}^{N_{J}} F_{i}\left(t_{j}+i H, \bar{x}\left(t_{j}+i H\right)\right) \\
\text { en }\left(u_{k}, \bar{x}\left(t_{j}+i H\right)\right) & \\
\text { sujeto a } & \end{cases} \\
& \begin{array}{ll}
\bar{x}\left(t_{j}+H\right)=x_{j}+H f\left(t_{j}+H, \bar{x}\left(t_{j}+H\right), u_{1}\right) & \\
\bar{x}\left(t_{j}+i H\right)=\bar{x}\left(t_{j}+(i-1) H\right)+H f\left(t_{j}+i H, \bar{x}\left(t_{j}+i H\right), u_{i}\right) & 1<i \leq N_{u} \\
\bar{x}\left(t_{j}+i H\right)=\bar{x}\left(t_{j}+(i-1) H\right)+H f\left(t_{j}+i H, \bar{x}\left(t_{j}+i H\right), u_{N_{u}}\right) & N_{u}<i \leq N_{J}
\end{array} \\
& u_{k} \in K \\
& 1 \leq k \leq N_{u} \\
& 1 \leq i \leq N_{J}
\end{aligned}
$$

En caso de tomar $T=h, N_{J}=1, N_{u}=1$, obtendríamos el algoritmo original.

Los ensayos realizados muestran que, en efecto, una adecuada elección del parámetro $T$ puede mejorar en gran medida los resultados obtenidos. Los parámetros $N_{J}$ y $N_{u}$, por otra parte, pueden hacer que el número de variables a optimizar aumente notablemente, por lo que deben seleccionarse con cuidado. En la Figura 6.6 se muestran los resultados de una sencilla simulación en la que se busca que el submarino realice un cambio de rumbo de $25^{\circ}$ en un tiempo de 50 segundos. Para ello se emplea el algoritmo anteriormente descrito empleando como único control la deflexión del rudder, $\delta_{r}$, con $h=1 \mathrm{~s}, N_{J}=1, N_{u}=1$ y diferentes valores de $T$. La velocidad de avance es de 5 nudos. La parte superior de la Figura 6.6 muestra la evolución del ángulo $\psi$ para los diferentes valores de $T$ considerados. En la parte inferior se muestra la variable de control, la deflexión del rudder. Como puede verse, la elección de $T=h$ (línea violeta) conduce a valores extremos de la variable de control que acaban por causar una enorme inestabilidad. Este efecto se ve mitigado conforme aumenta $T$ (línea roja, $T=3$ ), hasta que para $T=5$ (línea negra), se alcanza un excelente seguimiento de la referencia. Si continúan tomándose valores crecientes del parámetro $T$, sin embargo, el rendimiento del sistema se ve deteriorado de manera progresiva (línea cían, $T=10$, línea azul, $T=20$ ). Este sencillo ejemplo da muestra de la importancia que, en ocasiones, puede tener una correcta elección de los parámetros $T, N_{J}$ y $N_{u}$.

En el presente trabajo nos limitaremos a la formulación original propuesta en la Sección 6.2. En el Capítulo 8, Sección 8.1.2, emplearemos este esquema para el control en forma feedback de los procesos de soplado y ventilación durante una maniobra de ascenso de emergencia. 

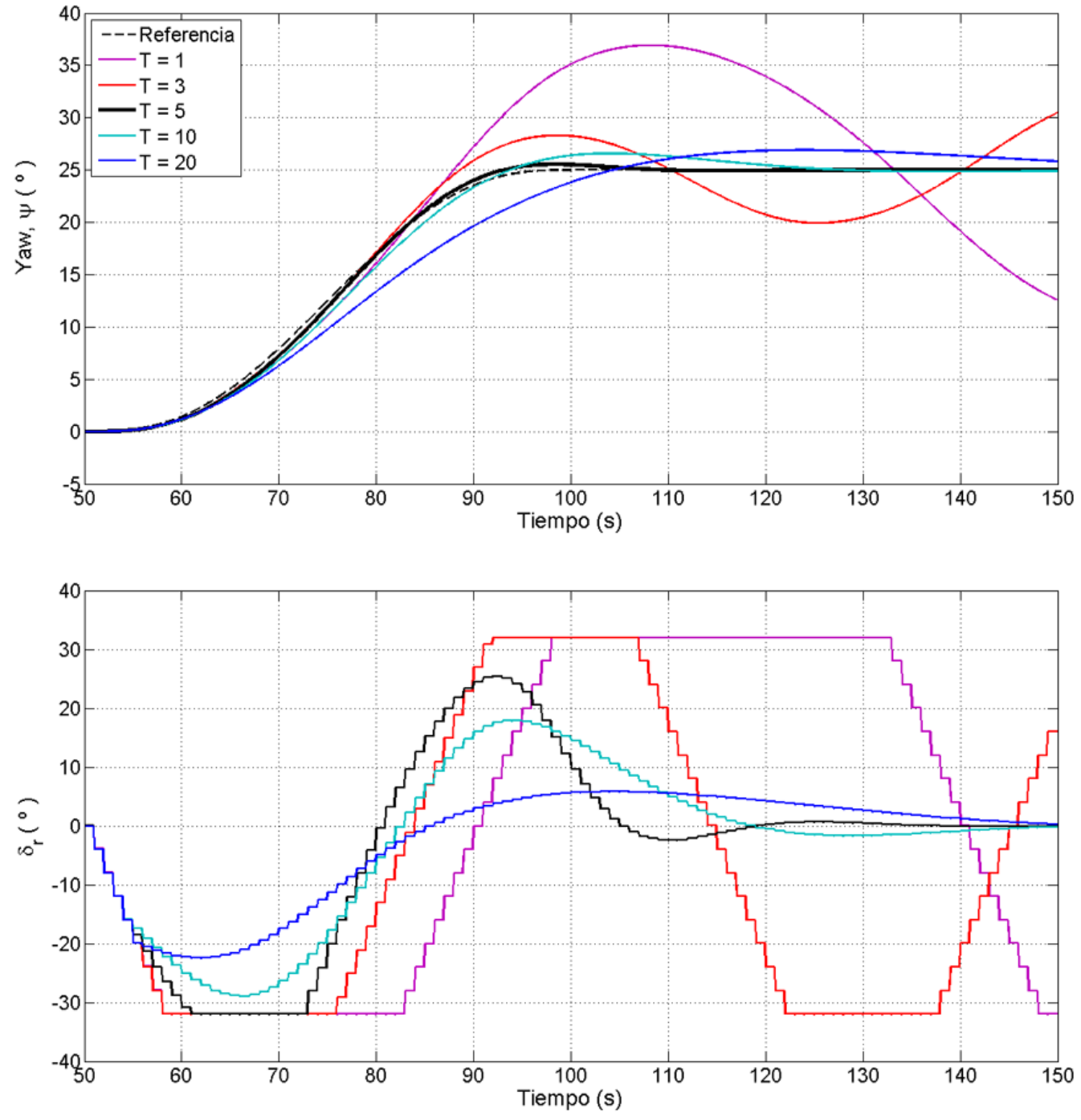

Figura 6.6: Comparación de los resultados obtenidos para un cambio de rumbo a $5 \mathrm{kn}$ empleando $h=1$ y $T=1$ (línea violeta), $T=3$ (línea roja), $T=5$ (línea negra), $T=10$ (línea cían) y $T=20$ (línea azul). 


\section{Control en modo deslizante de sistemas no lineales}

Hemos visto en capítulos anteriores cómo es posible calcular trayectorias óptimas para una determinada maniobra que pueden ser seguidas en forma feedback corrigiendo errores de medición, en el modelado, o la acción de perturbaciones externas. En ocasiones, sin embargo, la situación es ligeramente diferente: no existe una trayectoria como tal, sino que el objetivo es mantener el vehículo estable ante perturbaciones de gran tamaño como las descritas en el Capítulo 2. Éste el caso de la maniobra conocida como hovering, en la que el submarino debe mantener estable su posición de forma estática, en ausencia de velocidad de avance. En estas circunstancias, la resolución en cada intervalo temporal de un problema de programación matemática no lineal como $\left(\mathrm{NPP}_{h}^{j}\right)$ puede requerir un excesivo tiempo de cálculo o incluso no converger a una solución válida. Es deseable, por tanto, disponer de estrategias de control que puedan ser empleadas en tiempo real y garanticen un buen comportamiento ante perturbaciones de gran tamaño.

Una de las estrategias más extendidas para la resolución de este tipo de problemas es el control en modo deslizante (sliding mode control, SMC). Esta aproximación ha sido aplicada con éxito en el control de diversos vehículos autónomos submarinos y ha sido identificada, en efecto, como una de las estrategias de control más prometedoras para hacer frente a grandes perturbaciones o incertidumbres [LAM99]. Si bien el SMC es a menudo aplicado sobre un modelo lineal [DK04, RHMB05], las características del modelo dinámico del submarino, en concreto su alta no linealidad y el elevado nivel de acoplamiento entre los distintos grados de libertad, nos inducen a pensar que esta aproximación puede ser inapropiada para el presente 
caso. En lugar de una aproximación lineal, proponemos el uso de la linealización feedback que, como veremos, permite obtener una relación lineal de forma exacta.

Esta técnica es descrita en la Sección 7.1 mientras que el control en modo deslizante se describe en la Sección 7.2. La Sección 7.3, por último, trata el uso de estas técnicas para el control del vehículo mediante el soplado y ventilación de los tanques de lastre.

\subsection{Linealización feedback}

En los últimos años la linealización feedback ha despertado un creciente grado de interés, hasta convertirse en una de las herramientas fundamentales para el control de sistemas no lineales. Como tal, puede encontrarse una completa descripción de esta técnica en cualquier texto clásico de control no lineal, como [Kha01, SL91]. El mayor rigor matemático puede encontrarse en [Isi95].

La idea tras la linealización feedback es aplicar una transformación algebraica capaz de convertir un sistema no lineal -o parte de él- en un sistema lineal, de forma que puedan aplicarse para su control las contrastadas técnicas de control de sistemas lineales. Es importante destacar que esta linealización es exacta, y por tanto muy diferente de la obtenida mediante la bien conocida técnica de considerar una aproximación lineal en el entorno de un punto.

Cuando la linealización feedback busca obtener una relación lineal entre el control y el estado hablamos de linealización input-state, mientras que si se busca una relación lineal entre el control y la salida del sistema hablamos de linealización input-output. Puesto que la linealización input-output es suficiente para nuestros objetivos, en las siguientes secciones nos centraremos en la segunda de estas técnicas. La Sección 7.1.1 cubre el caso de sistemas con una única entrada y una única salida (Single Input Single Output, SISO), mientras que la Sección 7.1.2 extiende los resultados a sistemas con múltiples entradas y múltiples salidas (Multiple Input Multiple Output, MIMO).

\subsubsection{Linealización input-output de sistemas de una entrada y una salida (SISO)}

Decimos que un sistema no lineal está en forma afín cuando es lineal en el control. De este modo, un sistema de una entrada y una salida en forma afín toma la forma:

$$
\begin{aligned}
\dot{\mathbf{x}} & =\mathbf{f}(\mathbf{x})+\mathbf{g}(\mathbf{x}) u \\
y & =h(\mathbf{x})
\end{aligned}
$$

con $\mathbf{x} \in \mathbb{R}^{n}$ y $\mathbf{f}, \mathbf{g}$ funciones de $\mathbb{R}^{n}$ en $\mathbb{R}^{n}$. Como es usual, $u$ denota el control, mientras que $y$ es la salida del sistema. Es decir, en este caso no estamos interesados en controlar el estado del sistema, $\mathbf{x}$, sino una función escalar de éste, $y=h(\mathbf{x})$. 
El objetivo de la linealización input-output es buscar una ley de control no lineal de la forma $\alpha(\mathbf{x})+\beta(\mathbf{x}) v$ tal que la relación entre la salida $y$ y el nuevo control $v$ es lineal. El siguiente ejemplo proporciona una idea intuitiva de este proceso:

Ejemplo 1. Considérese el sistema

$$
\begin{aligned}
& \dot{x}=x^{2}+\sin x+x u \\
& y=x
\end{aligned}
$$

Es inmediato ver que la elección de la ley de control $u=\frac{1}{x}\left(v-x^{2}-\sin x\right)$ cancela de forma exacta las no linealidades, convirtiendo el sistema en $\dot{y}=\dot{x}=v$.

Por supuesto, la facilidad con la que se ha obtenido una expresión para la ley de control se debe a la sencillez del ejemplo. Para obtener una metodología general es necesario, en primer lugar, introducir algunos conceptos.

Definición 7.1. Sean $h: \mathbb{R}^{n} \rightarrow \mathbb{R} y \mathbf{f}: \mathbb{R}^{n} \rightarrow \mathbb{R}^{n}$, derivables. Llamamos derivada de Lie de $h$ con respecto a $\mathbf{f}$ a la función escalar $L_{f} h=\nabla h \mathbf{f}$.

Las sucesivas derivadas de Lie pueden definirse de forma recursiva como

$$
L_{f}^{i} h=L_{f}\left(L_{f}^{i-1} h\right)=\nabla\left(L_{f}^{i-1} h\right) \mathbf{f} .
$$

Del mismo modo, dado un segundo campo vectorial $\mathbf{g}, L_{g} L_{f} h$ es:

$$
L_{g} L_{f} h=\nabla\left(L_{f} h\right) \mathbf{g} .
$$

Definición 7.2. Se dice que el sistema (7.1) tiene grado relativo $r$ en un punto $\mathbf{x}^{0}$ si

1. $L_{g} L_{f}^{i} h(\mathbf{x})=0$ para todo $\mathbf{x}$ en un entorno de $\mathbf{x}^{0}$ y para $i=0, \ldots, r-2$.

2. $L_{g} L_{f}^{r-1} h\left(\mathbf{x}^{0}\right) \neq 0$.

Del mismo modo, si estas condiciones se cumplen para todo $\mathbf{x}$ en un conjunto $\Omega$, se dice que (7.1) tiene grado relativo $r$ en $\Omega$. Para mayor detalle acerca de estos conceptos se remite al lector a [Isi95, SL91].

Sea el sistema (7.1) y sea $\Omega \subseteq \mathbb{R}^{n}$ el conjunto en el que la variable de estado $\mathrm{x}$ toma valores. Diferenciando la salida $y$ con respecto al tiempo se obtiene

$$
\dot{y}=L_{f} h+L_{g} h u .
$$

Si $\left(L_{g} h\right)(\mathbf{x}) \neq 0 \forall \mathbf{x} \in \Omega$, entonces el control

$$
u=\frac{1}{L_{g} h}\left(v-L_{f} h\right)
$$


transforma (7.1) en el sistema lineal

$$
\dot{y}=v \text {. }
$$

Si $\left(L_{g} h\right)(x) \equiv 0$, diferenciando de nuevo la salida se obtiene

$$
\ddot{y}=L_{f}^{2} h+\left(L_{g} L_{f} h\right) u \text {. }
$$

De forma general, si $r$ es el grado relativo del sistema, mediante el control

$$
u=\frac{1}{L_{g} L_{f}^{r-1} h}\left(v-L_{f}^{r} h\right)
$$

se obtiene:

$$
y^{(r)}=v
$$

Una vez hecho esto, resulta sencillo aplicar la teoría de control lineal para controlar el sistema (7.3). En concreto, una ley de la forma

$$
v=-k_{1} y^{(r-1)}-\cdots-k_{r-2} \dot{y}-k_{r-1} y
$$

estabiliza asintóticamente (7.3) alrededor del origen (las definiciones de estabilidad y estabilidad asintótica de un sistema pueden encontrarse en detalle, por ejemplo, en [Kha01, SL91]).

El desarrollo anterior muestra que, de forma intuitiva, puede definirse el grado relativo como el número de veces que es necesario diferenciar la salida $y$ para que el control $u$ aparezca de forma explícita.

Es importante destacar que, si bien (7.4) estabiliza asintóticamente (7.3), para que la ley de control (7.2) estabilice asintóticamente el sistema original (7.1), el comportamiento del resto de variables cuando la salida $y$ es nula (lo que se conoce como zero dynamics), debe cumplir condiciones adicionales. En concreto, el comportamiento de estas variables debe ser estable. Los detalles pueden encontrarse en [Isi95, SL91].

\subsubsection{Sistemas con múltiples entradas y múltiples salidas (MIMO)}

Considérese ahora el caso de un sistema con $m$ entradas y $p$ salidas,

$$
\begin{aligned}
\dot{\mathbf{x}} & =\mathbf{f}(\mathbf{x})+\sum_{i}^{m} g_{i}(\mathbf{x}) u_{i} \\
y_{1} & =h_{1}(\mathbf{x}) \\
& \vdots \\
y_{p} & =h_{p}(\mathbf{x}) .
\end{aligned}
$$

Se dice que este sistema tiene un vector grado relativo $\left(r_{1}, \ldots, r_{p}\right)$ en un punto $\mathbf{x}_{0}$ si se cumplen las dos condiciones siguientes: 
1. $L_{g_{j}} L_{f}^{k} h_{i}(x)=0, \forall 1 \leq i \leq p, \forall 1 \leq j \leq m, \forall k<r_{i}-1$ y para todo $\mathbf{x}$ en un entorno de $\mathbf{x}_{0}$.

2. El rango de la matriz

$$
A(x)=\left[\begin{array}{ccc}
L_{g_{1}} L_{f}^{r_{1}-1} h_{1}(x) & \ldots & L_{g_{m}} L_{f}^{r_{1}-1} h_{1}(x) \\
L_{g_{1}} L_{f}^{r_{2}-1} h_{2}(x) & \ldots & L_{g_{m}} L_{f}^{r_{2}-1} h_{2}(x) \\
\vdots & \ddots & \vdots \\
L_{g_{1}} L_{f}^{r_{p}-1} h_{p}(x) & \ldots & L_{g_{m}} L_{f}^{r_{p}-1} h_{p}(x)
\end{array}\right]
$$

en $\mathbf{x}=\mathbf{x}^{0}$ es igual a su número de columnas.

Bajo estas condiciones, el sistema (7.5) puede expresarse como

$$
\left[\begin{array}{c}
y_{1}^{r_{1}} \\
\vdots \\
y_{p}^{r_{p}}
\end{array}\right]=\left[\begin{array}{c}
L_{f}^{r_{1}} h_{1} \\
\vdots \\
L_{f}^{r_{p}} h_{p}
\end{array}\right]+A(x)\left[\begin{array}{c}
u_{1} \\
\vdots \\
u_{m}
\end{array}\right]
$$

de donde puede obtenerse la expresión de $\left[u_{1}, \ldots, u_{m}\right]$ que reduce el sistema a $p$ ecuaciones de la forma

$$
y_{i}^{\left(r_{i}\right)}=v_{i}, \quad i=1, \ldots, p
$$

\subsection{Control en modo deslizante}

El control en modo deslizante proporciona una aproximación sistemática al control de sistemas tanto lineales como no lineales en presencia de errores o incertidumbres. Esta técnica ha sido aplicada con éxito en el control de diversos vehículos submarinos [DK04, RHMB05, HL93].

El control en modo deslizante se basa en transformar un problema de orden $n$ en un problema de primer orden de forma que, para este problema transformado, pueden obtenerse resultados tan precisos como se desee (a costa de aumentar la acción de control necesaria) en presencia de incertidumbres arbitrariamente grandes. El SMC es tratado en detalle en [SL91]. Una completa introducción con gran rigor matemático, incluyendo más recientes desarrollos y algunas interesantes aplicaciones, puede encontrarse en [PU11].

Considérese el sistema SISO

$$
y^{(n)}=f(x)+b(x) u
$$

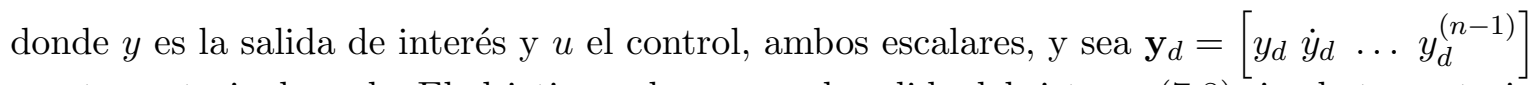
una trayectoria deseada. El objetivo es lograr que la salida del sistema (7.8) siga la trayectoria 
$\mathbf{y}_{d}$ en presencia de imprecisiones en el modelo. En concreto, supondremos que las funciones $f(x)$ y $b(x)$ no son exactamente conocidas, aunque se conocen cotas sobre el error cometido en su estimación.

Si consideramos la variable $\mathbf{y}=\left[\begin{array}{llll}y & \dot{y} & \ldots & y^{(n-1)}\end{array}\right]$, podemos expresar el error en el seguimiento de $\mathbf{y}_{d}$ como $\tilde{\mathbf{y}}=\mathbf{y}-\mathbf{y}_{d}=\left[\begin{array}{lll}\tilde{y} & \dot{\tilde{y}} \ldots & \tilde{y}^{(n-1)}\end{array}\right]$. Definamos la superficie $S(t)$ en el espacio de estado $\mathbb{R}^{n}$ por la ecuación escalar $s(\mathbf{y} ; t)=0$, donde

$$
s(\mathbf{y} ; t)=\left(\frac{d}{d t}+\lambda\right)^{n-1} \tilde{y}
$$

siendo $\lambda$ una constante positiva, de forma que, por ejemplo, para $n=3$ se tiene

$$
s=\ddot{\tilde{y}}+2 \lambda \dot{\tilde{y}}+\lambda^{2} \tilde{y}
$$

Esta nueva variable $s$ representa una medida escalar del seguimiento de la variable $\mathbf{y}_{d}$, de forma que un problema de orden $n$ es reducido a uno de primer orden; mantener la cantidad escalar $s$ igual a cero. Seguir la trayectoria $\mathbf{y}_{d}$ equivale a permanecer sobre la superficie $S(t)$ (sliding surface).

Este problema de primer orden, más sencillo que el original, puede resolverse eligiendo una ley de control $u$ tal que fuera de $S(t)$

$$
\frac{1}{2} \frac{d}{d t} s^{2} \leq-\eta|s|
$$

donde $\eta$ es una constante positiva.

Esto implica que todas las trayectorias apuntan a la superficie $S(t)$, como muestra la Figura 7.1 (izquierda), y, una vez alcanzada ésta, permanecen sobre ella. En concreto, (7.10) garantiza (la demostración puede encontrarse en [SL91]) que, si en $t=0$ el sistema se encuentra fuera de la superficie $S(t)$, ésta se alcanza en un tiempo $t \leq|s(t=0)| / \eta$. Cuando el sistema evoluciona sobre la superficie $S(t)$ se dice que está en modo deslizante o sliding mode.

Una vez reducido el problema de orden $n$ a un problema de primer orden a través de $s$, la obtención de la ley de control $u$ se compone de dos etapas. En primer lugar, ha de elegirse una ley de control que garantice (7.10). Para que esta condición se mantenga en presencia de incertidumbres o perturbaciones, sin embargo, esta ley de control ha de ser discontinua a través de $S(t)$. Puesto que las transiciones entre las expresiones a ambos lados de $S(t)$ son necesariamente imperfectas -el cambio no puede ser instantáneo y $s$ no se conoce con absoluta exactitud-, la implementación de esta ley discontinua causará las oscilaciones a muy alta frecuencia conocidas como chattering (Figura 7.1, derecha). Para evitar este fenómeno, en una segunda etapa, la ley de control es suavizada en un entorno de $S(t)$.

Se denomina control equivalente, $u_{e q}$, al control que garantiza que, en ausencia de incertidumbres, una vez sobre la superficie $s=0$ el sistema permanece sobre ella. Para ello, es 

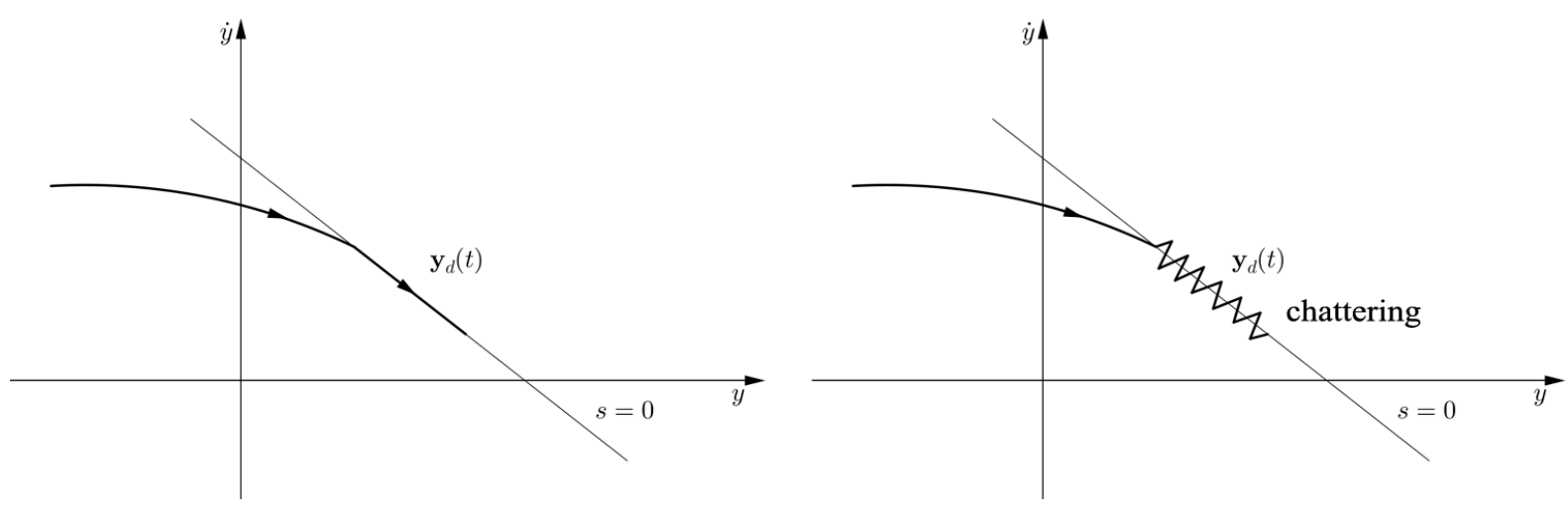

Figura 7.1: Comportamiento de un sistema bajo SMC (se representa un sistema con $n=2$ ). Oscilaciones conocidas como chattering.

necesario que se verifique $\dot{s}=0$. Para $n=3$, es inmediato ver que

$$
\dot{s}=y^{(3)}-y_{d}^{(3)}+2 \lambda \ddot{\tilde{y}}+\lambda^{2} \dot{\tilde{y}}=f+u_{e q}-y_{d}^{(3)}+2 \lambda \ddot{\tilde{y}}+\lambda^{2} \dot{\tilde{y}}=0
$$

luego

$$
u_{e q}=-f-y_{d}^{(3)}+2 \lambda \ddot{\tilde{y}}+\lambda^{2} \dot{\tilde{y}}
$$

Consideremos ahora el caso en que las funciones $f$ y $b$ no son exactamente conocidas. Sean $\hat{f}, \hat{b}$ y $\hat{u}_{e q}$, respectivamente, las estimaciones de $f$ y $b$ y la estimación del control equivalente obtenida a partir de $\hat{f}, \hat{b}$, y sean $F$ y $\beta$ cotas sobre el error cometido en la estimación de $f$ y $b$, de forma que

$$
\begin{aligned}
& |\hat{f}-f| \leq F, \\
& \beta^{-1} \leq \frac{b}{\hat{b}} \leq \beta .
\end{aligned}
$$

La ley de control

$$
u=\hat{u}_{e q}-k \operatorname{sign}(s),
$$

donde $\operatorname{sign}(\cdot)$ es la función signo y $k$ una constante positiva, garantiza el cumplimiento de (7.10) siempre y cuando la elección de $k$ sea tal que

$$
k \geq \beta(F+\eta)+(\beta-1)|\hat{u}| .
$$

Si no se considera error en la estimación de $b$, sino únicamente en la de $f$, bastaría con tomar

$$
k \geq(F+\eta) .
$$

Se remite al lector a [SL91] para los detalles sobre la obtención de las cotas (7.13) y (7.14).

Como se dijo con anterioridad, la presencia del término discontinuo $k \operatorname{sign}(s)$ supone que, una vez alcanzada la superficie $s=0$, el control tiende a oscilar con frecuencia infinita. Para 
evitar este fenómeno, es práctica habitual aproximar la función signo, en el interior de una capa límite de grosor $\Phi$, mediante la función continua sat $(s / \Phi)$ (Figura 7.2):

$$
\operatorname{sat}(s / \Phi)= \begin{cases}s / \Phi & \text { si }|s / \Phi| \leq 1 \\ \operatorname{sign}(s / \Phi) & \text { en caso contrario }\end{cases}
$$

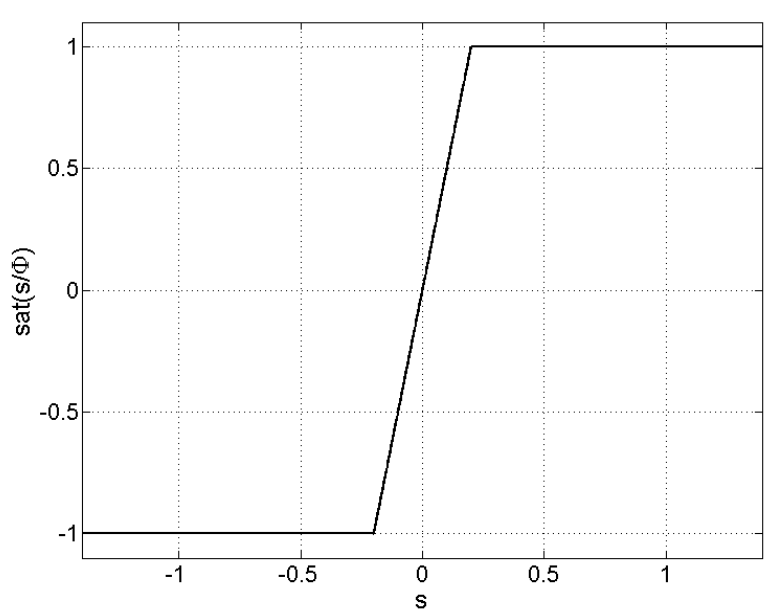

Figura 7.2: Representación de la función $\operatorname{sat}(\cdot)$ para $\Phi=0,2$.

Esto supone que el seguimiento de $s=0$ no se realiza de forma exacta sino con una determinada tolerancia que depende de $\Phi$. Se remite al lector a [SL91] para los detalles, así como una muestra de cómo puede tomarse un $\Phi$ variable con el tiempo.

\subsection{Control de los procesos de soplado y ventilación}

En las maniobras en las que estamos interesados, como el hovering (ver Capítulo 9), el objetivo es mantener una determinada cota. Resulta natural, por tanto, considerar una única salida, la cota $z$, y $m$ entradas, las aperturas de las válvulas de soplado y ventilación. Mediante manipulación algebraica, es sencillo expresar (4.5) en la forma (7.5)

$$
\begin{aligned}
\dot{\mathbf{x}} & =\mathbf{a}(\mathbf{x})+\sum_{i}^{m} b_{i}(\mathbf{x}) u_{i} \\
y & =h(\mathbf{x})=z
\end{aligned}
$$

donde, puesto que nos limitaremos a valores constantes de $\delta_{r}, \delta_{s}, \delta_{b}, n$-el control va a realizarse exclusivamente mediante el soplado y ventilación de los tanques-, se ha eliminado la dependencia explícita de la variable temporal.

El cálculo de $L_{b_{i}} L_{a}^{j}, j=1 \ldots r$ muestra que el sistema tiene grado relativo $r=3$. Sean 


$$
\begin{gathered}
F(x)=L_{a}^{r} h \text { y } \mathbf{G}(\mathbf{x})=\left[\begin{array}{lll}
L_{b_{1}} L_{a}^{r-1} h & \ldots & L_{b_{m}} L_{a}^{r-1} h
\end{array}\right] \text {. El sistema (7.16) puede expresarse como } \\
\qquad y^{(r)}=F(\mathbf{x})+\mathbf{G}(\mathbf{x})\left[\begin{array}{c}
u_{1} \\
\vdots \\
u_{m}
\end{array}\right]
\end{gathered}
$$

que puede reducirse a

$$
y^{(3)}=v
$$

mediante la ley de control

$$
\mathbf{u}=\mathbf{G}^{*}(\mathbf{x})(v-F(\mathbf{x}))
$$

donde $\mathbf{G}^{*}(\mathbf{x})$ es un vector tal que $\mathbf{G}(\mathbf{x}) \mathbf{G}^{*}(\mathbf{x})=1$.

Para garantizar que (7.19) es capaz de estabilizar el sistema completo (7.16), resta verificar, como se dijo en la Sección 7.1.1, que el sistema es estable en sus zero dynamics. En el presente caso, dada la complejidad del sistema, resulta extremadamente difícil probar esta estabilidad. Los resultados de simulación (véase el Capítulo 9), sin embargo, parecen indicar que el sistema es efectivamente estabilizado con éxito. Desde el punto de vista físico, el submarino en consideración es, hasta donde sabemos, esencialmente estable salvo en el movimiento vertical, donde existe la inestabilidad tratada en la Sección 2.2.1, que no supone ningún problema puesto que es precisamente el movimiento vertical el que está siendo estabilizado por (7.19).

Por supuesto, la exacta cancelación de las no linealidades para obtener (7.18) requiere un conocimiento perfecto de las funciones $\mathbf{a}(\mathbf{x})$ y $b_{i}(\mathbf{x})$, lo que no es posible en presencia de perturbaciones. Para hacer frente a las perturbaciones haremos uso del control en modo deslizante. El uso de esta estrategia de control en sistemas linealizados mediante linealización feedback se describe de forma más general, por ejemplo, en [FF91].

Sean $\mathbf{y}=\left[\begin{array}{llll}y & \dot{y} & \ldots & y^{(r-1)}\end{array}\right]$ el vector de salida, $\mathbf{y}_{d}=\left[\begin{array}{llll}y_{d} & \dot{y}_{d} & \ldots & y_{d}^{(r-1)}\end{array}\right]$ el vector de salida deseado e $\tilde{\mathbf{y}}=\mathbf{y}-\mathbf{y}_{d}=\left[\begin{array}{llll}\tilde{y} & \dot{\tilde{y}} & \ldots & \tilde{y}^{(r-1)}\end{array}\right]$ el error en la salida como se describieron en la Sección 7.2. Podemos definir $s$ como

$$
s=\ddot{\tilde{y}}+2 \lambda \dot{\tilde{y}}+\lambda^{2} \tilde{y} .
$$

Derivando respecto al tiempo la expresión anterior y teniendo en cuenta que en nuestro caso $y_{d}^{(3)}=0$ y en ausencia de perturbaciones $y^{(3)}=v$, el control equivalente es $v_{e q}=-2 \lambda \ddot{\tilde{y}}-\lambda^{2} \dot{\tilde{y}}$.

Sean $\widehat{F}(\mathbf{x}), \widehat{\mathbf{G}}(\mathbf{x})$ las estimaciones de $F(\mathbf{x})$ y $\mathbf{G}(\mathbf{x})$. Si la función signo es aproximada por la función $\operatorname{sat}(\cdot)$ como se describió con anterioridad, la ley de control propuesta es

$$
\mathbf{u}=\widehat{\mathbf{G}}^{*}(\mathbf{x})\left(-2 \lambda \ddot{\tilde{y}}-\lambda^{2} \dot{\tilde{y}}-k \operatorname{sat}(s / \Phi)-\widehat{F}(\mathbf{x})\right) .
$$

Si consideramos que no existen errores en el modelado y que la única fuente de incertidumbre son las perturbaciones externas (cambios en la flotabilidad y esfuerzos producidos 
por el oleaje), entonces $\widehat{\mathbf{G}}(\mathbf{x})=\mathbf{G}(\mathbf{x})$. Sea $\Delta \geq|F(\mathbf{x})-\widehat{F}(\mathbf{x})|$ una cota del error cometido en la estimación de $F(\mathbf{x})$, entonces (ver Sección 7.2) basta con tomar $k>\Delta$.

El valor de $\Delta$ para cambios en la densidad del agua puede ser fácilmente estimado considerando valores extremos de la variación de ésta. En el caso de los esfuerzos producidos por el oleaje, no puede darse un valor preciso de esta cota debido a la naturaleza aleatoria de estos esfuerzos. Sin embargo, sí pueden obtenerse estimaciones para un estado de mar dado.

Desde el punto de vista práctico, la obtención de $\widehat{F}(\mathbf{x}), \widehat{\mathbf{G}}(\mathbf{x})$ requiere el cálculo de los términos $L_{a}^{3} h$ y $L_{b_{i}} L_{a}^{2} h$. Desarrollando las derivadas de Lie obtenemos

$$
\begin{aligned}
& L_{a}^{3} h=\nabla(\nabla(\nabla h a) a) a, \\
& L_{b_{i}} L_{a}^{2} h=\nabla(\nabla(\nabla h a) a) b_{i} .
\end{aligned}
$$

Definamos $\alpha_{1}=\nabla(\nabla h a)=\nabla h \nabla a$, puesto que en este caso $\nabla^{2} h=0, \mathrm{y}$

$$
\alpha_{2}=\nabla(\nabla(\nabla h a) a)=\nabla\left(\alpha_{1} a\right)=a^{T} \nabla\left(\alpha_{1}^{T}\right)+\alpha_{1} \nabla a .
$$

Así, $L_{a}^{3} h=\alpha_{2}$ a y $L_{b_{i}} L_{a}^{2} h=\alpha_{2} b_{i}$.

Puesto que $h=z, \nabla h$ es nulo salvo en una componente, de modo que tanto $\alpha_{1}$ como $\nabla \alpha_{1}$ tienen expresiones que pueden ser fácilmente obtenidas de forma explícita. La expresión de $\nabla a$, presente en $\alpha_{2}$, por el contrario, es excesivamente compleja para su obtención por métodos analíticos. Se ha optado por tanto por su aproximación numérica mediante diferencias centrales. Puesto que nuestro objetivo es diseñar una ley de control robusta ante perturbaciones de gran tamaño, no es de esperar que el pequeño error numérico cometido en esta estimación sea de importancia.

En el Capítulo 9, Sección 9.3.2, se empleará este algoritmo para el control del hovering en presencia de perturbaciones externas. 


\section{Parte III}

\section{Resultados}





\section{Maniobras de ascenso de emergencia}

El procedimiento habitual, en caso de que una emergencia obligue a ascender a la superficie tan rápidamente como sea posible, consiste en aumentar al máximo la velocidad de giro de la hélice, emplear los timones para levantar la proa de forma que el submarino tome un ángulo de pitch positivo, y soplar los tanques de lastre para que la flotabilidad contribuya a llevar el vehículo a la superficie. Este soplado de los tanques es realizado en la actualidad de forma esencialmente manual, de modo que no existe control sobre el grado de apertura de las válvulas y los tanques son, en general, soplados de manera simultánea.

Durante el ascenso, el flujo incide sobre la vela de forma que, si existe un cierto roll inicial, ésta puede actuar como un rudder induciendo un momento de giro que tiende a hacer crecer este valor inicial. Este fenómeno ha sido tratado en [Wat01, Wat07, BGW09], y es causa de que se haya llegado a emerger con ángulos de hasta 25 grados. Es también sabido, además, que si el submarino emerge con un alto ángulo de roll puede experimentar grandes oscilaciones una vez en la superficie. Por supuesto, éste es un comportamiento indeseado, particularmente si la tripulación ha de atender al problema original que motivó el ascenso de emergencia.

Por estos motivos, resulta interesante comprobar si un adecuado control sobre el proceso de soplado, en solitario o en conjunción con ventilación y timones, puede ayudar a mejorar el comportamiento en este tipo de maniobras. Con este fin, se han simulado dos maniobras de ascenso de emergencia. En la primera de ellas, Sección 8.1, se emplea el control óptimo, como se describió en el Capítulo 5, para calcular una trayectoria óptima que mantenga un ángulo de roll tan pequeño como sea posible durante toda la maniobra (Sección 8.1.1). Posteriormente, 
en la Sección 8.1.2, se realiza el seguimiento de esta trayectoria óptima en presencia de errores en el modelado y la medición de la variable de estado mediante el algoritmo en lazo cerrado propuesto en el Capítulo 6. En la segunda de las maniobras -Sección 8.2- se simula un caso más desfavorable, en el que se produce la rotura del timón horizontal de popa. Las conclusiones se sintetizan en la Sección 8.3.

Las simulaciones numéricas han sido realizadas empleando Matlab ${ }^{1}$. La resolución de las ecuaciones de estado y del estado adjunto se ha llevado a cabo empleando la función ODE45, un método Runge-Kutta de orden 4,5 [SR97].

\subsection{Una maniobra de ascenso de emergencia}

En esta primera maniobra el submarino se encuentra a una cota inicial de $100 \mathrm{~m}$ con velocidad inicial de $2 \mathrm{~m} / \mathrm{s}$. En $t=0$, se realiza el soplado de los tanques $2-5$ con el fin de llevar el submarino a la superficie. En la Sección 8.1.1 compararemos los resultados obtenidos mediante la forma tradicional de proceder (sin control sobre la apertura de las válvulas de soplado) y el control óptimo, según se describió en el Capítulo 5, del proceso de soplado o los procesos de soplado y ventilación considerados de forma conjunta. Posteriormente, en la Sección 8.1.2, trataremos de seguir en forma feedback la trayectoria óptima calculada en este último caso empleando el algoritmo propuesto en el Capítulo 6.

\subsubsection{Análisis en lazo abierto}

Se ha simulado esta maniobra para tres diferentes escenarios:

Escenario 8.1: Maniobra estándar, similar al modo en que este tipo de maniobras son realizadas en la actualidad. La profundidad inicial es de $100 \mathrm{~m}$ y la velocidad inicial 2 $\mathrm{m} / \mathrm{s}$. En $t=0$, los timones horizontales de popa y proa son fijados a -20 y 20 grados respectivamente y la velocidad de giro de la hélice se eleva hasta las 2, $5 \mathrm{rps}$. Al mismo tiempo, los tanques de lastre del 2 al 5 son simultáneamente soplados con la mitad de la máxima intensidad (esto corresponde a $s_{i}=0,5$ en nuestro modelo). Las válvulas de ventilación permanecen cerradas $\left(\bar{s}_{i}=0\right)$ durante la maniobra al completo. La simulación concluye cuando el vehículo alcanza una profundidad de $10 \mathrm{~m}$ (una cota arbitrariamente pequeña para la que puede asumirse que se ha alcanzado la superficie).

\footnotetext{
${ }^{1} \mathrm{htt}: / /$ www.mathworks.com
} 
En resumen:

$$
\begin{array}{rlrl}
\delta_{r}(t) & =0 & & \\
\delta_{s}(t) & =-20 & & \\
\delta_{b}(t) & =20 & & \\
n(t) & =2,5 & & \\
s_{i}(t) & =0,5, \quad i=1 \ldots 4 & \\
\bar{s}_{i}(t) & =0, \quad i=2 \ldots 5 &
\end{array}
$$

Es necesario destacar que, pese a que nos referimos a este escenario como maniobra estándar, el valor constante adoptado por las superficies de control es por supuesto una simplificación de lo que se haría en una maniobra real.

Escenario 8.2: La misma maniobra, con el algoritmo de control actuando de $t=0$ a $t=30$ segundos empleando exclusivamente las válvulas de soplado $\left(\mathbf{u}=\left[s_{2}, \ldots, s_{5}\right]\right)$. Para la inicialización del algoritmo se emplean los controles del Escenario 8.1. El algoritmo busca lograr tres objetivos principales:

- El submarino debe ascender en un tiempo similar al empleado en la maniobra estándar.

- El ángulo de pitch final debe estar alrededor de los 20 grados y nunca por encima de 25 grados.

- El ángulo de roll debe permanecer tan cercano a cero como sea posible durante toda la maniobra.

Escenario 8.3: Igual al Escenario 8.2, incorporando el uso de las válvulas de ventilación junto a las de soplado $\left(\mathbf{u}=\left[s_{2}, \ldots, s_{5}, \bar{s}_{2}, \ldots, \bar{s}_{5}\right]\right)$.

Para los escenarios 8.2 y 8.3 se han empleado los siguientes parámetros:

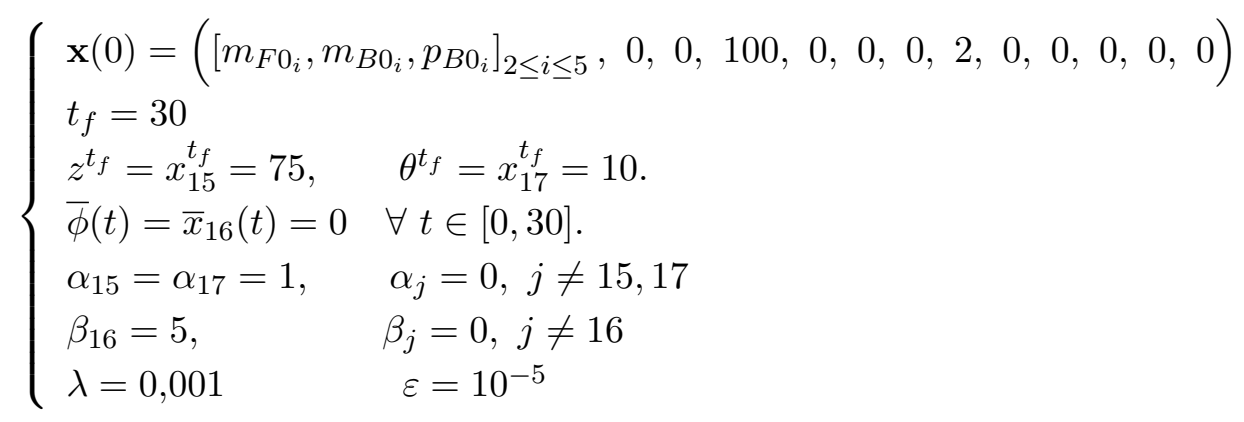

donde la masa de aire inicial en las botellas es $m_{F 0_{i}}=237,8376 \mathrm{~kg}$, la masa inicial de aire en los tanques es $m_{B 0_{i}}=0,0126 \mathrm{~kg}$ y la presión inicial en los tanques es $p_{B 0_{i}}=1,0846 \cdot 10^{6} \mathrm{~Pa}$. Tras $t=30 \mathrm{~s}$, la simulación continúa con controles constantes iguales a los empleados para la inicialización hasta que el submarino alcanza una cota de $10 \mathrm{~m}$.

Los resultados se muestran en las figuras 8.1-8.4. En la Figura 8.1 se representan la evolución de la cota, el pitch y el roll para los tres escenarios contemplados. No se han 
incluido el resto de variables de estado por no ser directamente relevantes para la maniobra en consideración. La comparación entre la línea roja discontinua (maniobra estándar) y la línea azul (Escenario 8.2) muestra que, para el Escenario 8.2, se ha logrado cumplir los tres objetivos marcados: el tiempo necesario para alcanzar la superficie es sólo unos segundos mayor que en la maniobra estándar, el pitch final está cerca de los 20 grados, y el ángulo de roll se ha reducido significativamente con respecto a la maniobra estándar. En efecto, el Escenario 8.1 muestra ángulos alrededor de los 3-4 grados durante la mayor parte de la maniobra mientras que para el Escenario 8.2, tras un pico inicial de 2 grados, el ángulo de roll se mantiene en valores notablemente bajos. Los resultados del Escenario 8.3 (línea negra) muestran que el comportamiento puede aún mejorarse incorporando las válvulas de ventilación a la variable de control. Aunque desde el punto de vista práctico puede no ser conveniente emplear las válvulas de ventilación durante un ascenso de emergencia y no es, desde luego, una práctica común, se han empleado en el Escenario 8.3 con el fin de explorar en su conjunto las capacidades del algoritmo propuesto. La Tabla 8.2 recoge el valor de la función coste obtenida en los tres escenarios.

\begin{tabular}{lccc}
\hline \hline Escenario & 8.1 & 8.2 & 8.3 \\
\hline \hline Coste & 1211,447 & 129,0241 & 125,1811 \\
\hline \hline
\end{tabular}

Tabla 8.1: Valor del coste obtenido con controles óptimos y estándar.

Las variables de control se muestran en las figuras 8.2 (válvulas de soplado) y 8.3 (válvulas de ventilación). Como puede verse, la tendencia del submarino a girar alrededor de su eje longitudinal es compensada soplando más lastre de los tanques de estribor. Puede sorprender que los tanques 2 y 4 sean ventilados cuando aún no contienen aire y que, durante ciertos periodos de tiempo, un mismo tanque tenga sus válvulas de soplado y ventilación abiertas de forma simultánea, pero hay que destacar que abrir la válvula de ventilación cambia la presión contra la que se sopla. Esto parece propiciar transiciones más suaves. En efecto, aunque llevar a cabo simultáneamente dos acciones de control aparentemente opuestas puede parecer ineficiente, el uso de las válvulas de ventilación parece mejorar los resultados obtenidos empleando únicamente el soplado. En este sentido, los resultados de la presente simulación representan un óptimo sin tener en cuenta consideraciones ingenieriles. De este modo es posible alcanzar una mejor comprensión de estas maniobras y sus dificultades asociadas. Desde el punto de vista práctico, pensando en la posible implementación en un vehículo real, puede tenerse en cuenta cualquier consideración ingenieril mediante su incorporación en la función coste.

La evolución del coste a lo largo del proceso iterativo se muestra, para el Escenario 8.3, en la Figura 8.4. Como puede verse, el algoritmo muestra una convergencia exponencial. Como es usual en este tipo de algoritmos, el resultado final depende de la inicialización. Se probaron diferentes inicializaciones dando lugar a diferentes controles óptimos, lo que parece sugerir la existencia de diferentes mínimos locales y/o globales. 

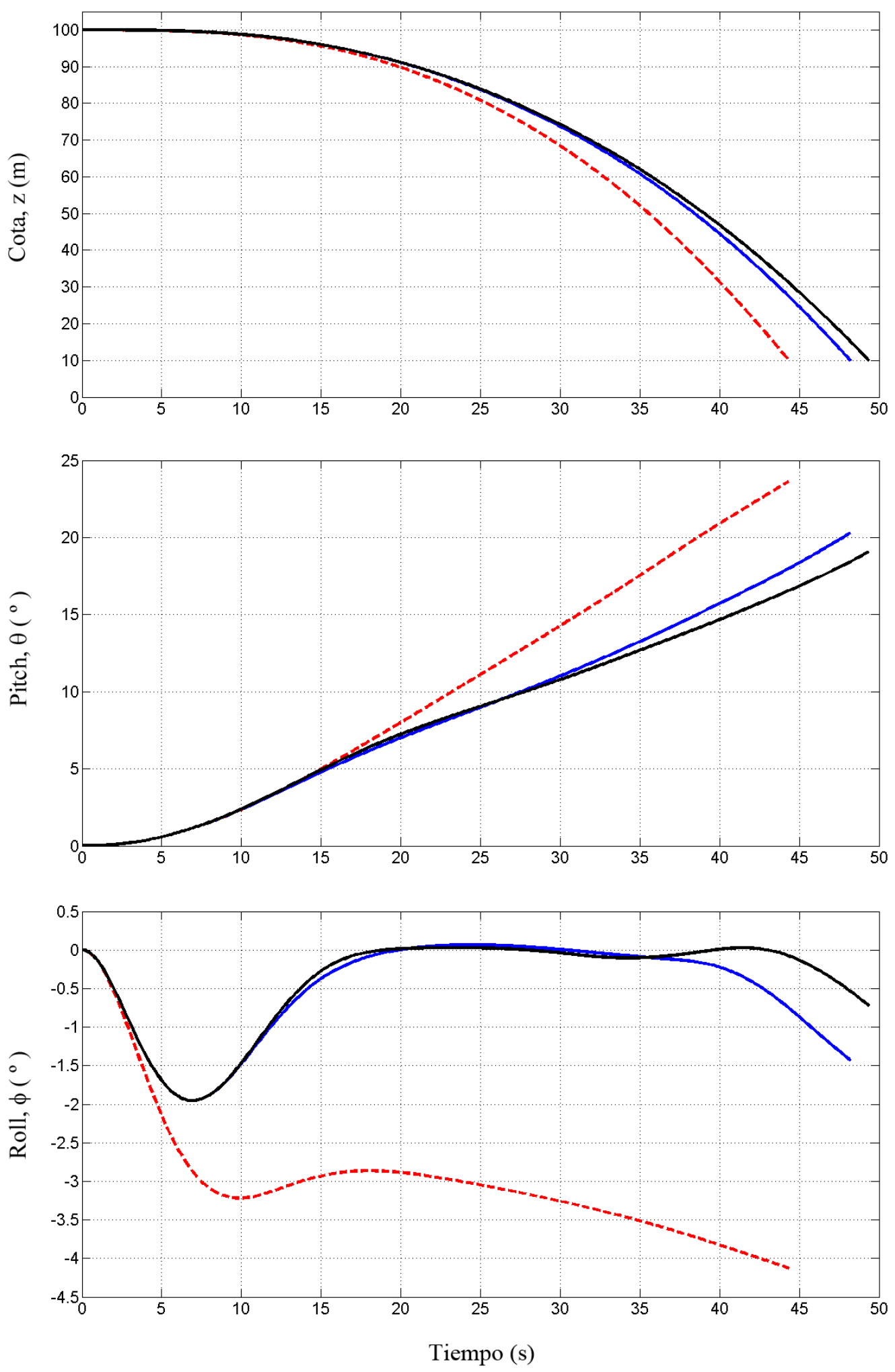

Figura 8.1: Evolución de la cota (arriba) y los ángulos de pitch (centro) y roll (abajo). El Escenario 8.1 se muestra en línea roja discontinua, el Escenario 8.2 en azul y el Escenario 8.3 en línea sólida negra. 

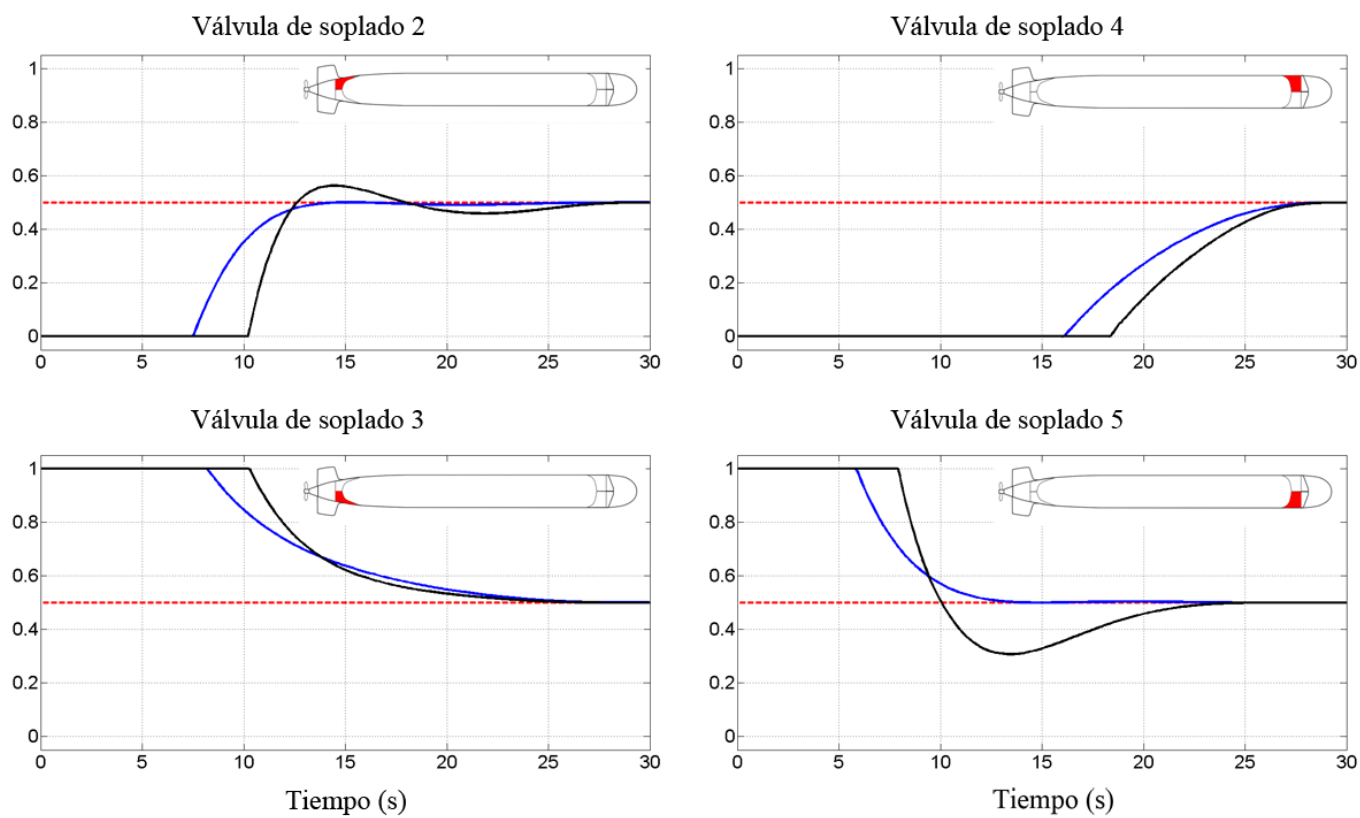

Figura 8.2: Apertura de las válvulas de soplado. Escenario 8.1, línea roja discontinua; Escenario 8.2, línea azul; Escenario 8.3, línea negra.)
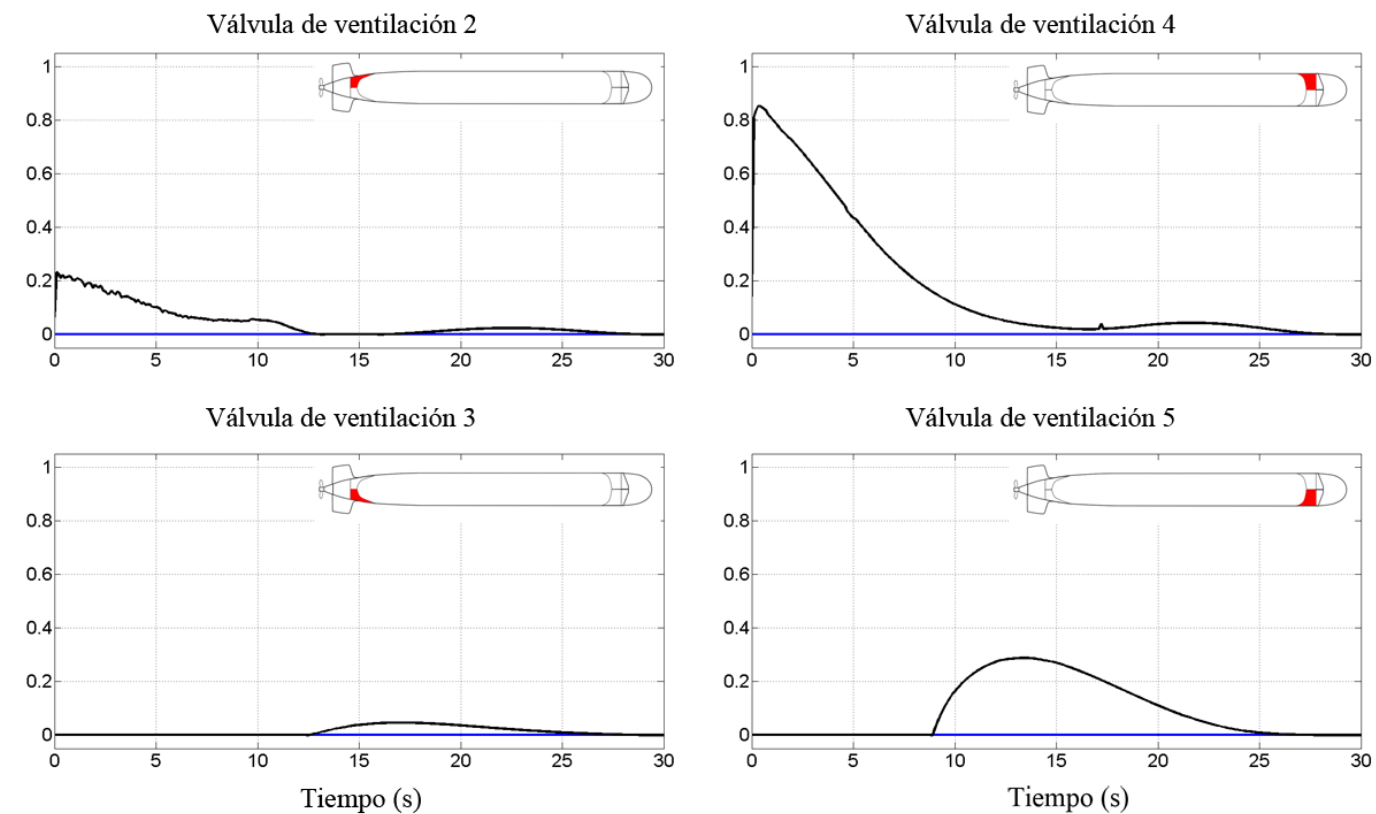

Figura 8.3: Apertura de las válvulas de ventilación. Escenario 8.1, línea roja discontinua; Escenario 8.2, línea azul; Escenario 8.3, línea negra. 


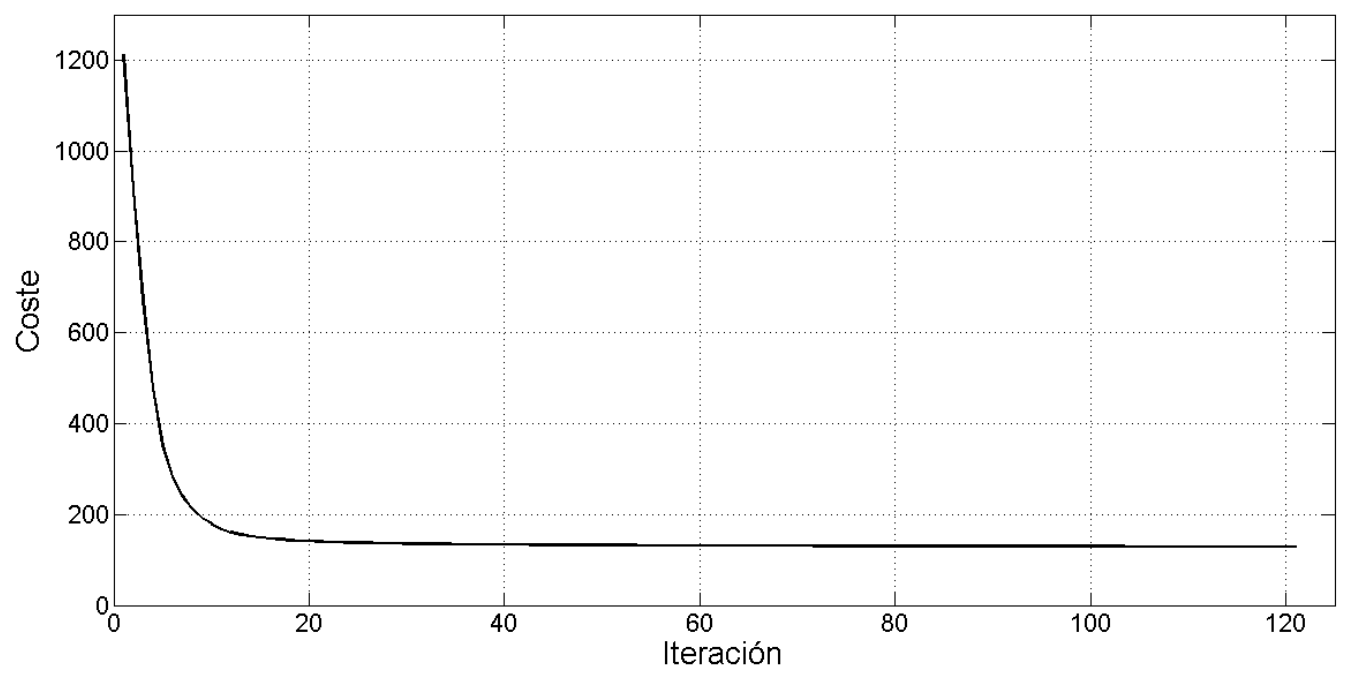

Figura 8.4: Evolución del coste para el Escenario 8.3.

\subsubsection{Control feedback}

Nuestro objetivo, ahora, es seguir en forma feedback la trayectoria óptima calculada en la sección anterior, en concreto la obtenida para el Escenario 8.3, en presencia de imprecisiones en el modelado y la medición. Emplearemos para ello el algoritmo de control en lazo cerrado descrito en el Capítulo 6.

Sea $\mathbf{x}_{\text {opt }}(t)$ la trayectoria óptima a seguir. Tal y como se describió en el Capítulo 6, tendremos el problema:

$$
\begin{cases}\text { Minimizar en } \mathbf{u}: & J(\mathbf{u})=\int_{0}^{t_{f}}\left(\mathbf{x}(t)-\mathbf{x}_{\text {opt }}(t)\right)^{2} d t \\ \text { sujeto a } & \mathbf{A}(\mathbf{x}(t)) \dot{\mathbf{x}}(t)=\mathbf{f}(t, \mathbf{x}(t), \mathbf{u}(t)) \\ & \mathbf{x}(0)=\mathbf{x}^{0} \\ & 0 \leq s_{i}(t) \leq 1,2 \leq i \leq 5 \\ & 0 \leq \bar{s}_{i}(t) \leq 1,2 \leq i \leq 5 .\end{cases}
$$

Este trajectory tracking problem puede resolverse del siguiente modo:

a. Dividir el intervalo $\left[t_{0}, t_{f}\right]$ en $N$ subintervalos temporales iguales $\left[t_{j}, t_{j}+h\right]$ con $h=$ $\left(t_{f}-t_{0}\right) / N$.

b. Durante el primer subintervalo $\left[t_{0}, t_{0}+h\right]$, aplicar los controles óptimos en lazo abierto. 
c. En $t=t_{0}+h$ medir el valor real de las variables de estado.

d. Empleando estas mediciones y la trayectoria óptima $\mathbf{x}_{o p t}$, resolver el problema de programación matemática no lineal descrito en forma general como $\left(\mathrm{NPP}_{h}^{j}\right)$ obteniendo los controles para el subintervalo $\left[t_{0}+h, t_{0}+2 h\right]$.

e. Repetir los pasos c y d para cada uno de los subintervalos temporales.

En este esquema es necesario, como vemos, medir el valor real de las variables de estado en cada nodo temporal $t_{j}$. A efectos de simulación, estos valores medidos (simulados) se obtienen integrando numéricamente la ley de estado (4.5). Para simular la presencia de errores en la medición se ha añadido ruido blanco a la medida de cada una de las variables de estado relevantes: se ha considerado un error de $\pm 2 \mathrm{~m}$ en la posición del vehículo y de $\pm 0,25$ grados en su orientación. Así mismo, se ha simulado la presencia de errores en el modelado suponiendo que el valor real del máximo flujo de aire desde las botellas es $\dot{m}_{F m a x}$ real $=-35 \mathrm{~kg} / \mathrm{s}$ en lugar de $\operatorname{los} \dot{m}_{F \max }=-20 \mathrm{~kg} / \mathrm{s}$ considerados en el modelado; es decir, que el flujo de aire desde las botellas fue subestimado durante el modelado. De esta forma, el algoritmo de control emplea el valor $\dot{m}_{F \max }$ mientras que $\dot{m}_{F m a x, r e a l}$ es empleado para la resolución de la ley de estado obteniendo los valores de las variables de estado que son posteriormente perturbadas como se ha descrito anteriormente. Estos datos perturbados son los empleados como los valores reales de las variables de estado en el paso c.

Para esta simulación tomaremos un periodo de muestreo de $h=2 \mathrm{~s}$. En cada $t_{j}$, el correspondiente problema de programación matemática no lineal se ha resuelto empleando el Method of Moving Asymptotes (MMA) [Sva87], un procedimiento iterativo en el que, en cada iteración, se genera una aproximación del problema original estrictamente convexa que es resuelta mediante un método dual.

Desde el punto de vista práctico, la velocidad a la que las variables de control pueden cambiar está limitada por las características de los sistemas mecánicos reales implicados, en este caso las válvulas de soplado y ventilación y sus actuadores. Por este motivo, puede ser deseable limitar la diferencia entre los controles calculados para dos intervalos temporales consecutivos. Esto no implica ningún cambio en el esquema de control propuesto. Si no se consideran límites en la velocidad de cambio, resolveremos $\left(\mathrm{NPP}_{h}^{j}\right)$ buscando controles $u^{j} \in \mathcal{K}=[0,1]^{8} \forall j$; si se tienen en cuenta estos límites, el conjunto $\mathcal{K}$ cambiará en cada nodo temporal $j$. De este modo, $u_{i}^{j} \in\left[\operatorname{máx}\left(0, u_{i}^{j-1}-\Delta\right), \operatorname{mín}\left(u_{i}^{j-1}+\Delta, 1\right)\right]$, con $\Delta$ la máxima diferencia admisible.

Teniendo esto en cuenta, la maniobra se ha simulado considerando dos casos: el Escenario 8.4, donde no se consideran restricciones en la diferencia entre dos controles consecutivos, y el Escenario 8.5, donde esta diferencia se ha limitado a $\Delta=0,2$. Los resultados de ambos escenarios se muestran en las figuras 8.5-8.8. En las figuras 8.5 y 8.6 se representan los valores de la cota y los ángulos de pitch y roll obtenidos mediante los controles óptimos 
(línea discontinua), los controles óptimos en presencia de errores en el modelado (línea verde), y los controles en lazo cerrado bajo errores de medición y en el modelado (línea azul, Escenario 8.4, línea roja, Escenario 8.5). Los datos perturbados simulando los valores medidos de las variables de estado se muestran como asteriscos $(*)$. Las figuras 8.7 y 8.8 muestran las aperturas de las válvulas de soplado y ventilación respectivamente.

Como puede verse (figuras 8.5 y 8.6, línea verde), la presencia de errores en el modelado hace que el empleo de los timones óptimos calculados en lazo abierto produzca resultados notablemente alejados de la trayectoria óptima. El uso del algoritmo de control propuesto (Figura 8.5, línea azul, Figura 8.6, línea roja) logra, en cambio, un excelente seguimiento de la trayectoria óptima. En la Figura 8.7 (línea azul) podemos ver que el error cometido en el modelado es compensado con éxito reduciendo la apertura de las válvulas de soplado. Las aperturas de las válvulas de soplado 2 y 4 , sin embargo, muestran notables oscilaciones. Si bien estas oscilaciones parecen ser correctas desde el punto de vista de optimizar el comportamiento del sistema, pueden suponer un problema a la hora de llevar a la práctica estos controles en un sistema real. Estas consideraciones prácticas han sido tenidas en cuenta en el Escenario 8.5 (figuras 8.6-8.8, línea roja), en el que se han eliminado las oscilaciones a costa de obtener un seguimiento de la trayectoria ligeramente inferior.

En ausencia de errores, tanto de modelado como de medición, los resultados numéricos parecen indicar que el coste obtenido por el control en lazo cerrado converge al coste óptimo calculado en lazo abierto conforme el periodo de muestreo $h$ se aproxima a cero. La Tabla 8.2 muestra el valor de la función coste del problema original $\left(\mathrm{OCP}^{2}\right)$

$$
J(u)=(z(30)-75)^{2}+(\theta(30)-10)^{2}+5 \int_{0}^{30} \phi^{2}(t) d t
$$

para diferentes tamaños de paso temporales $h$ así como para la trayectoria óptima y la maniobra estándar.

\begin{tabular}{|l|c|}
\hline Maniobra estándar (sin control) & 1076,7204 \\
\hline Control óptimo (lazo abierto) & 141,8405 \\
\hline Control feedback $(h=5 \mathrm{~s})$ & 143,4100 \\
\hline Control feedback $(h=2 \mathrm{~s})$ & 142,6789 \\
\hline Control feedback $(h=1 \mathrm{~s})$ & 142,5862 \\
\hline Control feedback $(h=0,5 \mathrm{~s})$ & 142,4268 \\
\hline
\end{tabular}

Tabla 8.2: Valor de la función coste para diferentes periodos de muestreo.

Para concluir, y con el objetivo de comparar ambos métodos, se ha resuelto este mismo problema mediante el método de transcripción descrito en la Sección 6.3. Los resultados numéricos son muy similares, aunque la convergencia del método de transcripción es considerablemente más lenta, requiriendo hasta 10 veces más tiempo de cálculo. 

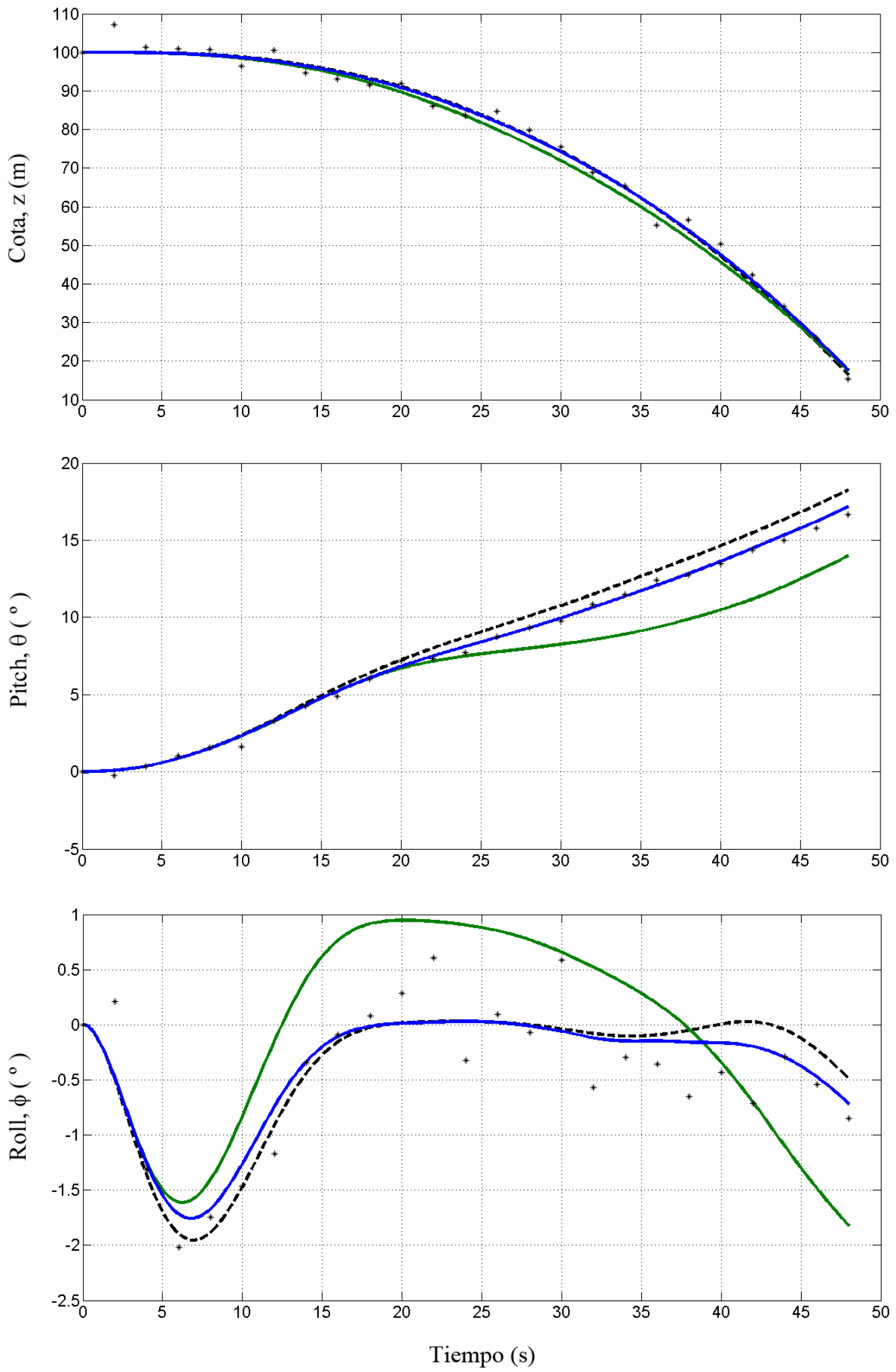

Figura 8.5: Escenario 8.4. Evolución de la cota (arriba) y los ángulos de pitch (centro) y roll (abajo) para la trayectoria óptima (línea discontinua), los controles óptimos en presencia de errores en el modelado (línea verde), y los controles feedback bajo errores de medición y en el modelado sin considerar límites en su tasa de cambio (línea azul). 

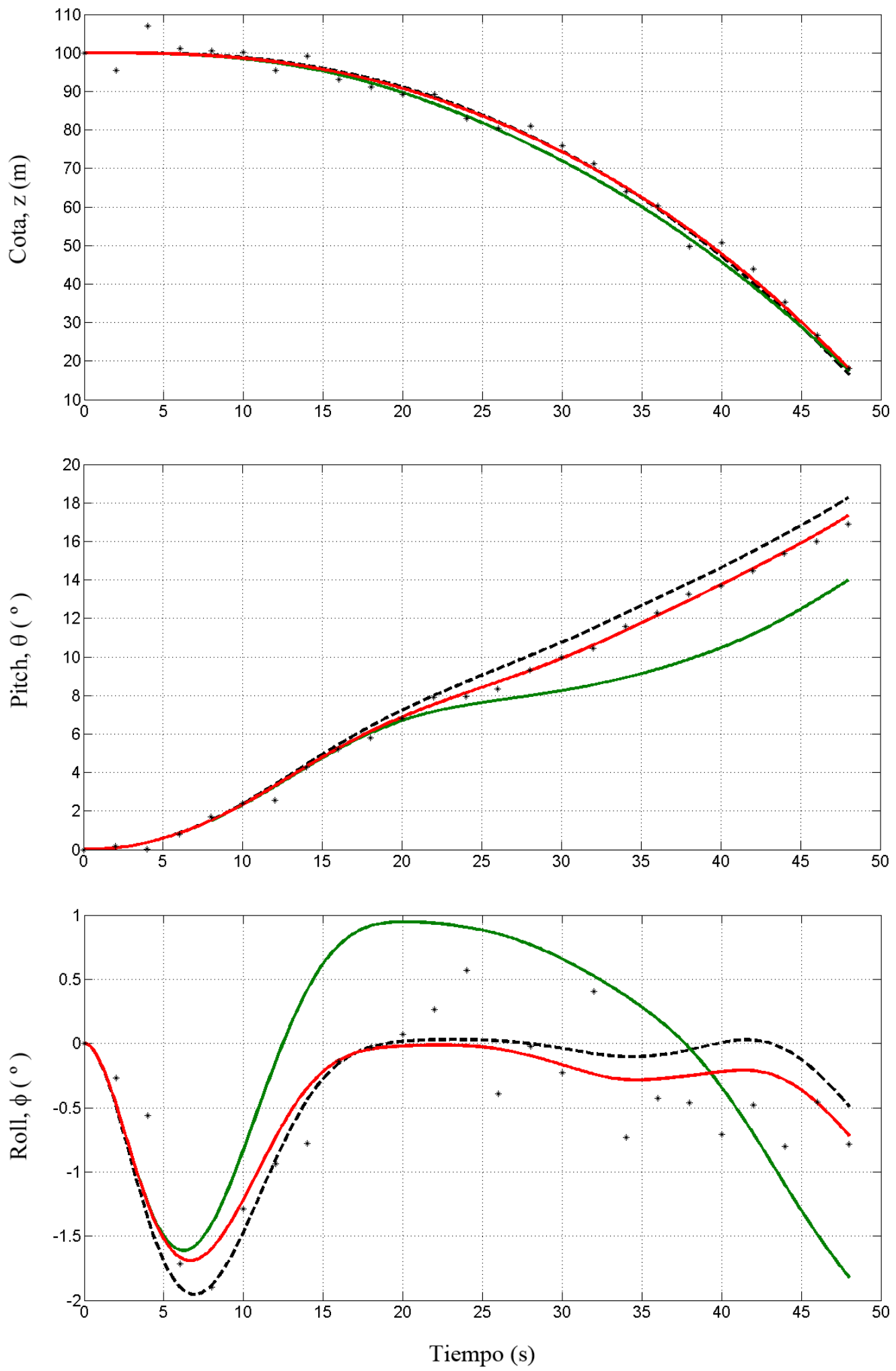

Figura 8.6: Escenario 8.5. Evolución de la cota (arriba) y los ángulos de pitch (centro) y roll (abajo) para la trayectoria óptima (línea discontinua), los controles óptimos en presencia de errores en el modelado (línea verde), y los controles feedback bajo errores de medición y en el modelado considerando una tasa de cambio limitada (línea roja). 

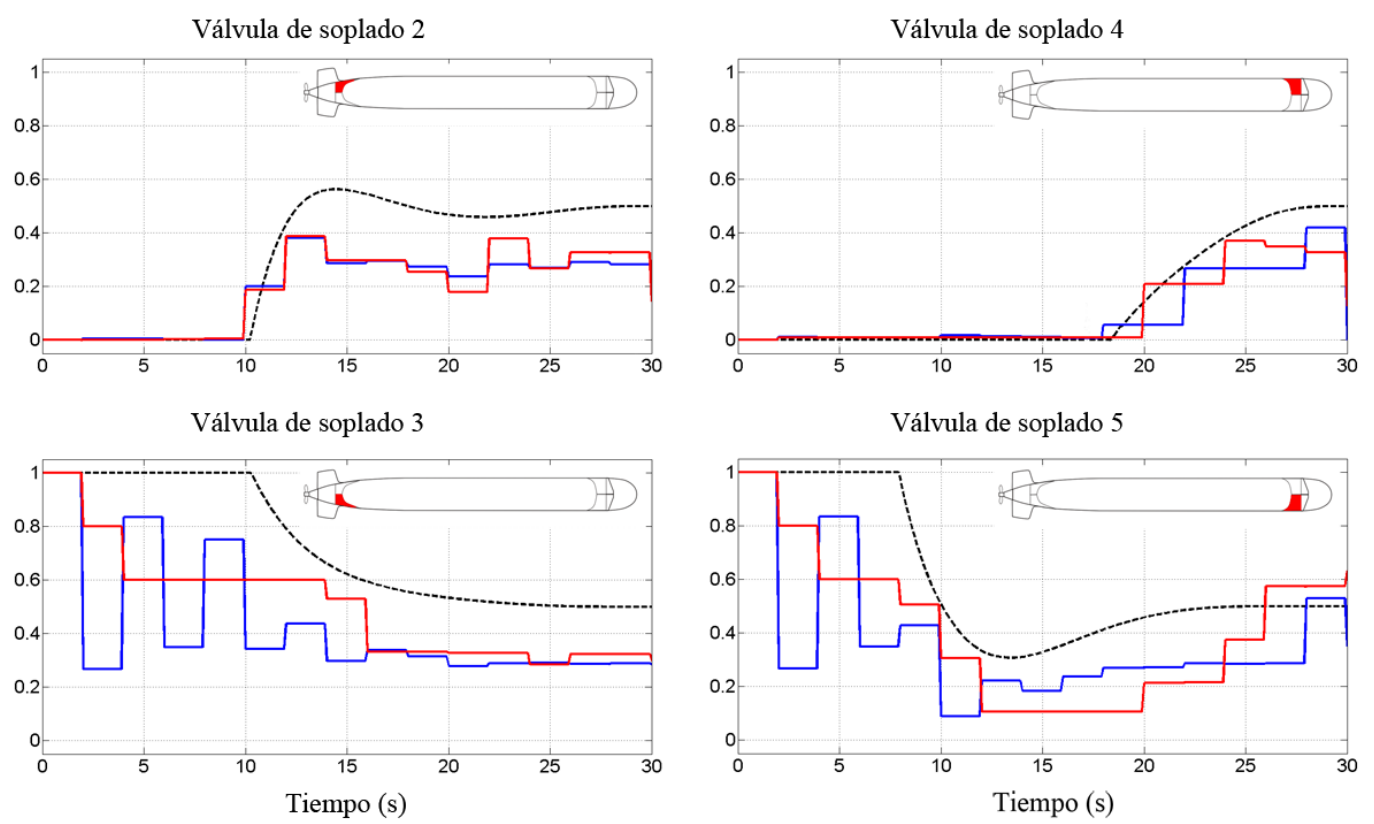

Figura 8.7: Apertura de las válvulas de soplado para la trayectoria óptima (línea discontinua), y los controles feedback (Escenario 8.4 en azul y Escenario 8.5 en rojo.)
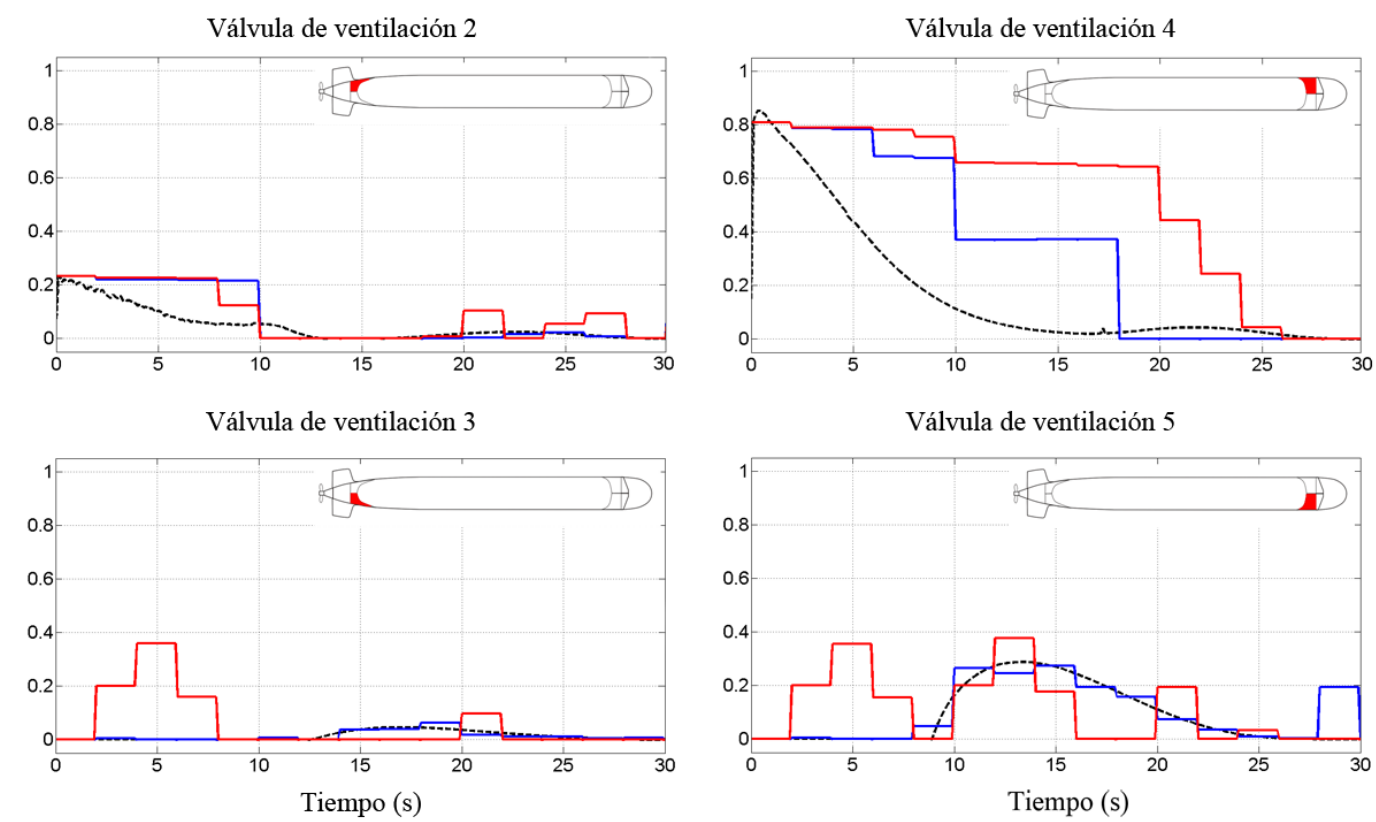

Figura 8.8: Apertura de las válvulas de ventilación para la trayectoria óptima (línea discontinua), y los controles feedback (Escenario 8.4 en azul y Escenario 8.5 en rojo). 


\subsection{Bloqueo del timón horizontal de popa durante un cambio de rumbo}

Una situación particularmente comprometida, por los elevados ángulos de roll que pueden llegar a alcanzarse, es la que se produce cuando el soplado de emergencia ha de realizarse durante un giro. En este caso, puesto que su valor inicial es ya considerable, el roll puede crecer hasta alcanzar valores fuera del rango admisible.

Consideremos la siguiente situación: el submarino navega a una cota de $200 \mathrm{~m}$ a $7 \mathrm{~m} / \mathrm{s}$, realizando un giro de forma que la deflexión del rudder es máxima. En ese instante, el timón horizontal de popa -stern plane- queda bloqueado comprometiendo la maniobrabilidad del vehículo y haciendo necesaria una maniobra de ascenso de emergencia. El hecho de no contar con el plano de popa para llevar el submarino a la superficie hace esta situación particularmente complicada. Una posible forma de afrontar esta maniobra es soplar los tanques de proa en primer lugar, de forma que ésta ascienda y el vehículo apunte hacia arriba, y posteriormente soplar también los tanques de popa hasta llevar el submarino a la superficie.

La forma habitual de realizar esta maniobra en un submarino como el P-650, con los timones de popa dispuestos en cruz, consiste, sin embargo, en emplear el plano vertical para obtener el pitch necesario. Para ello, se detiene la propulsión para reducir la velocidad -puede incluso frenarse activamente invirtiendo el sentido de giro de la hélice- $\mathrm{y}$ se opone el timón al flujo incidente llevándolo a su máxima deflexión. Una vez obtenido el pitch necesario, vuelve a activarse la propulsión con el fin de lograr de nuevo la velocidad de avance necesaria para alcanzar la superficie. No suele realizarse el soplado de los tanques a menos que la estrategia anteriormente descrita resulte insuficiente.

Emplearemos la primera de las alternativas mencionadas, pese a no ser la forma usual de proceder, puesto que plantea una situación muy desfavorable que nos permitirá explorar las capacidades del proceso de soplado como mecanismo de control.

Para la inicialización, por tanto, se soplarán primero los tanques de proa (tanques 4 y 5) y tras treinta segundos se iniciará el soplado de los tanques de popa (tanques 2 y 3). Este soplado se realiza con valor constante igual a la mitad de la máxima intensidad $\left(s_{i}=0,5\right)$. Esta inicialización, mostrada como línea roja discontinua en las figuras 8.9-8.11, produce, como podemos ver en la Figura 8.9, ángulos de roll de hasta 40 grados, un valor superior al admisible y cercano a suponer un riesgo para la tripulación. Para el algoritmo de control, se han empleado las válvulas de soplado y los timones disponibles de forma conjunta (ver Sección 5.2.1), mientras que la ventilación no se ha utilizado. Como podemos ver, Figura 8.9, línea azul, mediante el uso controlado del proceso de soplado se logra mantener este ángulo en el entorno de los \pm 10 grados, un valor perfectamente admisible, a la vez que se obtiene un tiempo de ascenso similar. 

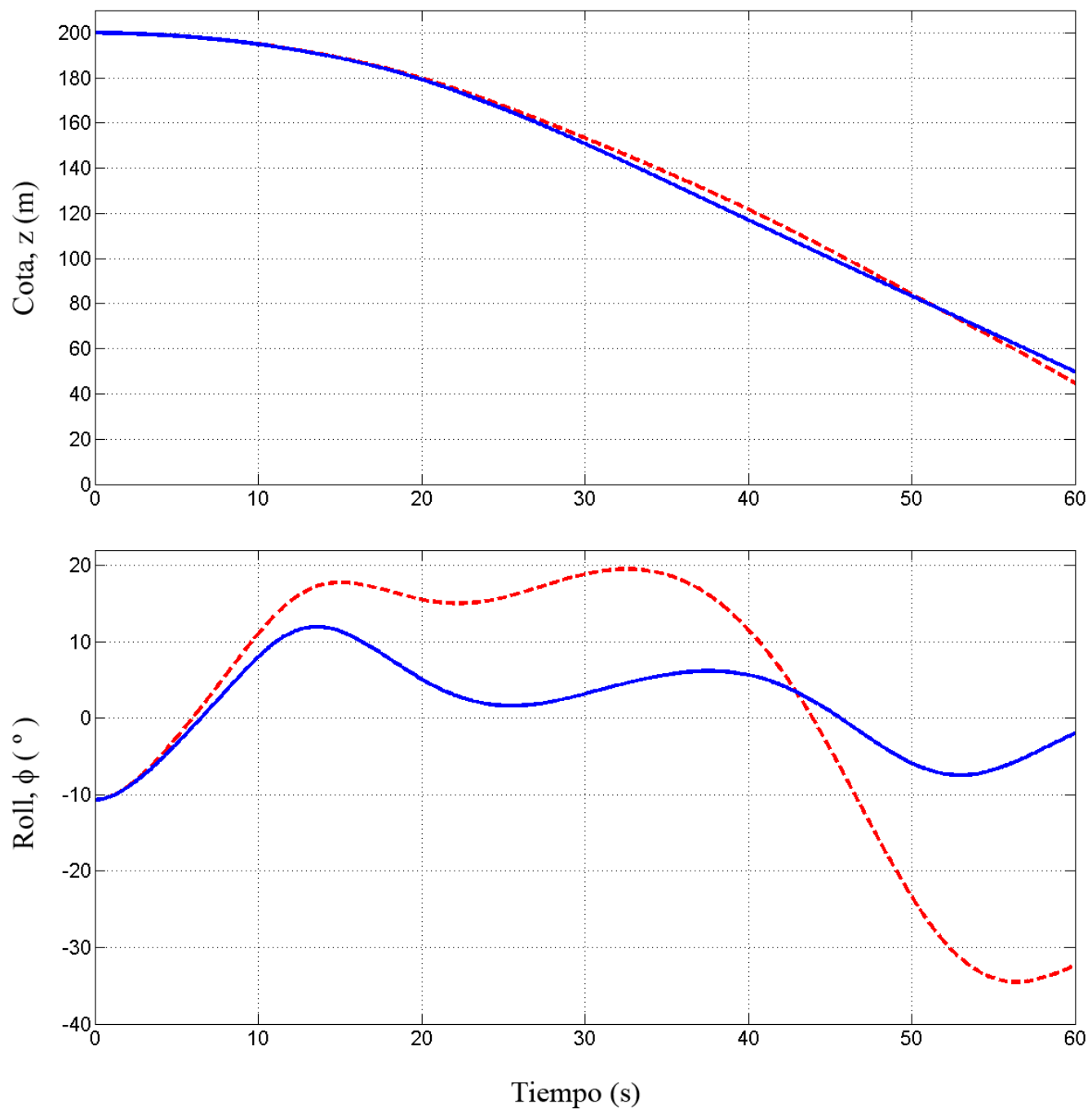

Figura 8.9: Evolución de la cota (arriba) y el ángulo de roll (abajo). La inicialización se muestra como línea roja discontinua mientras que la trayectoria óptima se muestra como línea sólida azul. 

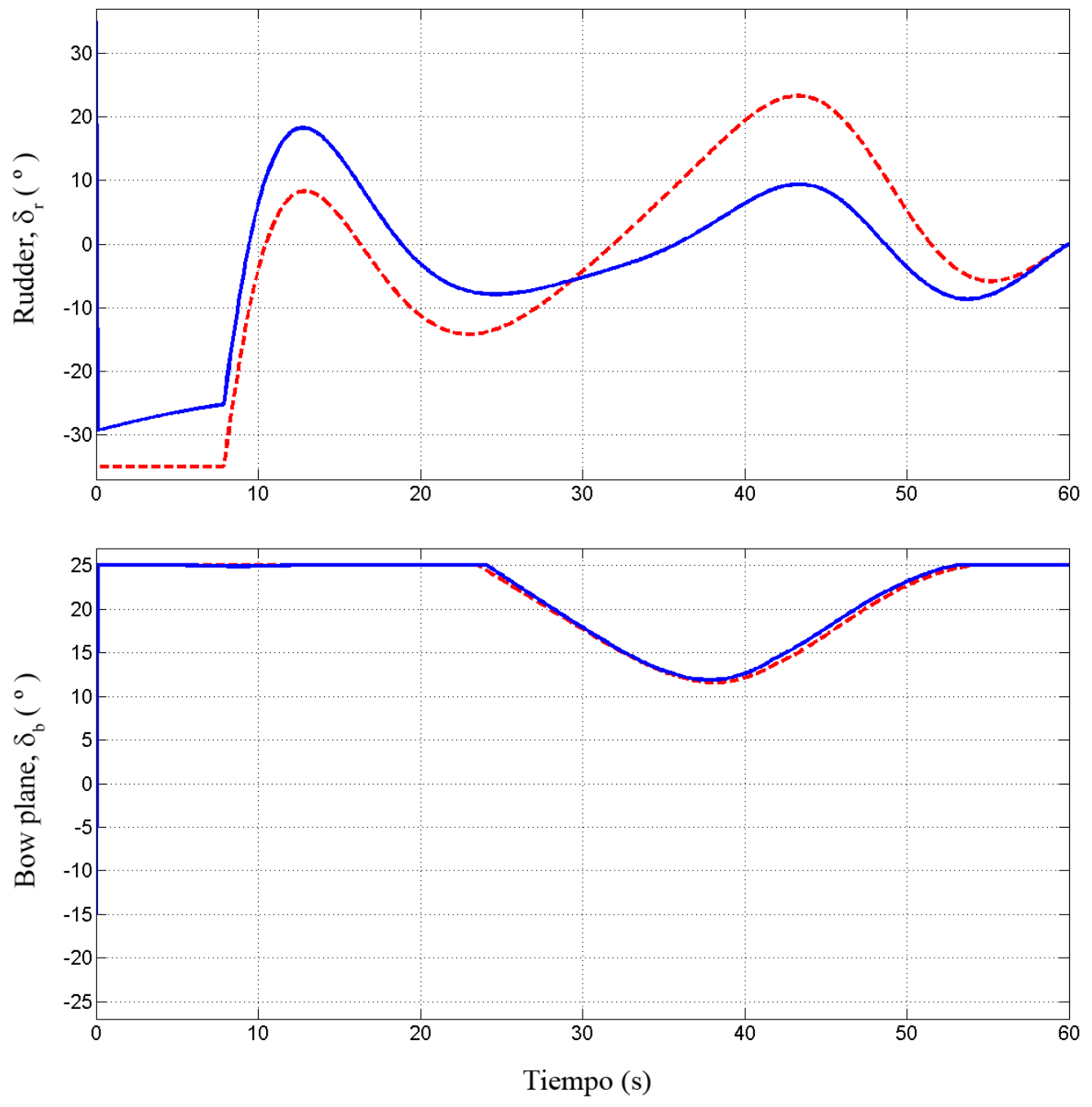

Figura 8.10: Deflexión del timón vertical (arriba) y el plano horizontal de proa (abajo). La inicialización se muestra como línea roja discontinua mientras que el control óptimo se muestra como línea sólida azul. 

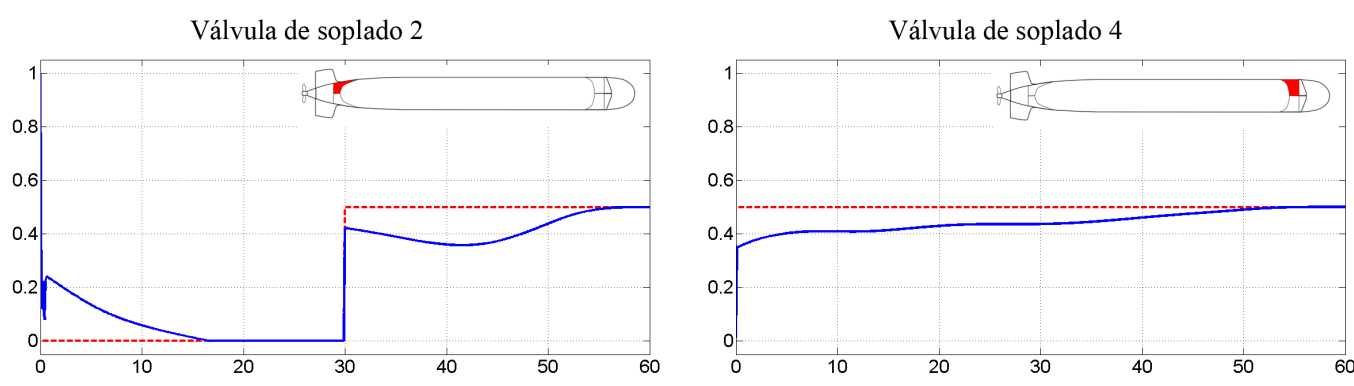

Válvula de soplado 3
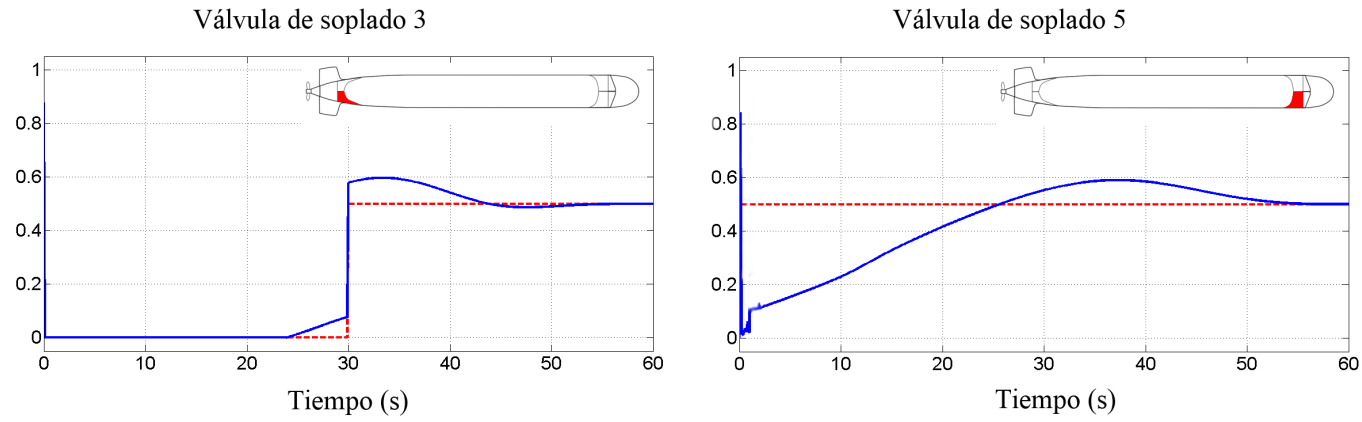

Figura 8.11: Apertura de las válvulas de soplado. La inicialización se muestra como línea roja discontinua mientras que los controles óptimos se muestran en línea sólida azul.

\subsection{Conclusiones}

Hemos mostrado cómo, con el control adecuado, el soplado diferencial de los tanques de lastre puede contribuir en gran medida a la mejora del comportamiento del submarino durante determinadas maniobras de emergencia. En la valoración de la posibilidad de implementar un sistema de control sobre los procesos de soplado/ventilación, actualmente realizados de forma eminentemente manual, intervienen, por otra parte, consideraciones prácticas y de seguridad que escapan al alcance y propósito de este trabajo.

En cualquier caso, la capacidad de simular este tipo de maniobras, poniendo a prueba diferentes estrategias, constituye una herramienta de enorme valor para el estudio de unas maniobras que, por sus características singulares, pueden difícilmente ser ensayadas en un buque real.

En el próximo capítulo estudiaremos el posible uso de los procesos de soplado y ventilación como un mecanismo de control que permita mantener la cota en ausencia de velocidad de avance, situación en la que los timones no son efectivos. 


\section{Maniobras a baja o nula velocidad. Hovering}

Los esfuerzos proporcionados por las superficies de control, el medio fundamental empleado para garantizar la maniobrabilidad y estabilidad del vehículo, son proporcionales al cuadrado de la velocidad de avance (véanse las ecuaciones (1.13)-(1.18)). Esto implica que, conforme la velocidad disminuye, disminuye también la efectividad de los timones. Cuando la velocidad se reduce por debajo de un cierto umbral, no es posible controlar el submarino. Puede encontrarse más detalle acerca de estas cuestiones en [Ova11].

Existen, sin embargo, situaciones en las que sería deseable ser capaces de mantener la estabilidad del submarino a muy reducidas o incluso nulas velocidades. En concreto, resulta de gran interés la maniobra conocida como hovering: la acción de mantener una cierta cota de forma estática, sin velocidad de avance.

El hovering es al mismo tiempo un desafío, debido a las muchas dificultades asociadas con el entorno subacuático, y una capacidad de gran importancia tanto para vehículos autónomos (Autonomous Underwater Vehicles, AUVs) como para submarinos tripulados. En los últimos tiempos se han diseñado diversos AUVs con esta capacidad (véase $\left[\mathrm{VDD}^{+} 10\right]$ para una revisión de esta materia). El hovering expande las posibilidades de los AUVs haciéndolos capaces de realizar tareas complejas que anteriormente sólo podían ser realizadas mediante el uso de vehículos operados de forma remota (Remotely Operated Vehicles, ROVs). En el caso de los submarinos tripulados, la capacidad de realizar esta maniobra de forma precisa resulta esencial, por ejemplo, para el despliegue de tropas especiales o el lanzamiento y recogida de AUVs, un asunto éste último que ha despertado de manera reciente un enorme grado de 
interés (véase por ejemplo [HB08, MCO11]). A medida que los conflictos de baja intensidad y las amenazas asimétricas se hacen cada vez más frecuentes, se ha hecho evidente que el uso de AUVs en misiones de vigilancia y reconocimiento, inteligencia, o minado y contraminado, podría no sólo mejorar el resultado de estas misiones sino reducir el riesgo de vidas humanas. Al mismo tiempo, los submarinos son considerados como la más prometedora plataforma para el despliegue de estos AUVs. En efecto, los submarinos proporcionan una discreción que difícilmente podría ser igualada por los barcos de superficie.

Si bien se han completado con éxito algunas pruebas de lanzamiento y recogida de AUVs posando el submarino en el fondo oceánico, es obvio que esta práctica presenta grandes limitaciones desde el punto de vista táctico. Por este motivo, resulta interesante desarrollar sistemas que permitan el mantenimiento de la cota y el trimado a muy baja o nula velocidad.

Desde el punto de vista tecnológico, en el caso de los AUVs el hovering se realiza habitualmente empleando propulsores $\left[\mathrm{CHCY03,} \mathrm{LPO}^{+} 11\right]$. Una estrategia alternativa, sin los grandes requerimientos energéticos asociados al uso de propulsores, consiste en controlar la flotabilidad del vehículo intercambiando agua entre el exterior y uno o varios tanques de lastre mediante el uso de bombas hidráulicas. Esta estrategia ha sido empleada con el fin de ahorrar energía $\left[\mathrm{VDD}^{+} 10\right]$ o en diseños de gran tamaño [TD07]. En el caso de los submarinos tripulados, el hovering se realiza tradicionalmente empleando bombas hidráulicas [YH10, YJ10], aunque es poca la información disponible en la literatura debido a la naturaleza militar de estos vehículos.

Nuestro objetivo es investigar el empleo de un sistema de hovering en el que un cierto número de tanques dispuestos al efecto es soplado y ventilado del mismo modo en que lo son los tanques de lastre principales. Estos últimos no son, debido a su gran tamaño, adecuados para el control de la flotabilidad necesario para el hovering. Consideraremos por tanto un conjunto de tanques específicamente adaptados para esta tarea. Los detalles pueden encontrarse en la Sección 9.1.

Con el fin de comparar los resultados obtenidos con este sistema con los que se obtendrían mediante el bombeo con bombas hidráulicas, la Sección 9.2 presenta un sencillo modelo para el control mediante bombeo. En la Sección 9.3 realizaremos simulaciones numéricas en la que se comparará la capacidad de ambos mecanismos de control para mantener la cota en presencia de perturbaciones como las descritas en el Capítulo 2. Se emplearán para ello los algoritmos de control óptimo (Sección 9.3.1) y control en modo deslizante (Sección 9.3.2) presentados en los capítulos 5 y 7 respectivamente. Por último, la Sección 9.4 presenta algunas conclusiones.

\subsection{Sistema de hovering}

Como hemos dicho, los tanques de lastre principales no son apropiados, por su gran tamaño y su importancia como mecanismo de seguridad, para el control de la flotabilidad 
necesario para llevar a cabo el hovering. Consideraremos en cambio un conjunto de tanques dispuestos específicamente para esta tarea. Puesto que en este caso no es necesario el control del roll, será suficiente con dos tanques, situados a proa y popa, con sus respectivas botellas y válvulas de soplado y ventilación. Tomando como punto de partida los tanques de lastre principales (Capítulo 3), se ha modificado la geometría de los tanques de hovering y sus sistemas de soplado y ventilación para adaptarlos a su nueva tarea. En concreto, se han realizado los siguientes cambios:

- Cada tanque de hovering tiene un volumen total de $4 \mathrm{~m}^{3}$ y está inicialmente a la mitad de su capacidad.

- El orificio de entrada/salida tiene un área de $0.1 \mathrm{~m}^{2}$.

- La sección del conducto de ventilación se ha reducido a un cuarto de su valor original.

- El máximo flujo de aire desde las botellas se ha reducido de $20 \mathrm{~kg} / \mathrm{s}$ a $2 \mathrm{~kg} / \mathrm{s}$.

Es importante destacar que estos son valores tentativos en el contexto de este estudio preliminar. Por supuesto, la implementación en un vehículo real requeriría seleccionar cuidadosamente el valor más apropiado de cada uno de estos parámetros.

Puesto que el número de tanques se ha visto reducido, tendremos ahora un total de 18 ecuaciones diferenciales ordinarias y un vector de estado

$$
\begin{aligned}
\mathbf{x}= & {[x, y, z, \phi, \theta, \psi, u, v, w, p, q, r, \ldots} \\
& \left.m_{F_{\text {stern }}}, m_{B_{\text {stern }}}, p_{B_{s t e r n}}, m_{F_{\text {bow }}}, m_{B_{b o w}}, p_{B_{b o w}}\right]
\end{aligned}
$$

compuesto por las variables de estado para los tanques de popa (stern) y proa (bow), la posición y orientación del vehículo $x, y, z$ y $\phi, \theta$ y $\psi$ y las velocidades lineales y angulares $u, v, w$ y $p, q, r$ (véanse los capítulos 1 y 3 para la descripción de estos conjuntos de variables).

Definamos $\Delta m_{\text {stern }}, \Delta m_{\text {bow }}$, las variaciones de masa en los tanques de popa y proa respectivamente, positivas cuando se añade masa. De forma similar a la descrita en la Sección 3.7 tenemos

$$
\Delta m_{\{\text {stern, bow }\}}(t)=\rho\left(V_{B 0}-V_{B_{\{\text {stern, bow }\}}}(t)\right)=\rho\left(V_{B 0}-\frac{m_{B_{\{\text {stern }, \text { bow }\}}}(t) R_{g} T_{B}}{p_{B_{\{\text {stern }, \text { bow }\}}}}\right) .
$$

El valor instantáneo de la masa del vehículo será

$$
m(t)=m_{0}+\Delta m_{\text {stern }}(t)+\Delta m_{\text {bow }}(t) .
$$

El resto de parámetros puede obtenerse de forma análoga (ver Sección 3.7). 


\subsection{Modelo matemático para el control mediante bombas hidráulicas}

Presentamos en esta sección un sencillo modelo para el control del hovering mediante bombeo con bombas hidráulicas. Emplearemos este modelo para contrastar los resultados obtenidos mediante bombeo y soplado/ventilación.

Para el sistema basado en bombeo el vector de estado es

$$
\mathbf{x}=\left[x, y, z, \phi, \theta, \psi, u, v, w, p, q, r, V_{\text {stern }}, V_{\text {bow }}\right]
$$

donde $V_{\text {stern }}$ y $V_{\text {bow }}$ son respectivamente los volúmenes de agua en los tanques de popa y proa. Consideraremos en este caso como variables de control $Q_{\text {stern }}, Q_{\text {bow }}$, los caudales suministrados por las bombas de los tanques de popa y proa. La variación en $V_{\text {stern }}, V_{\text {bow }}$ viene dada por estos caudales:

$$
\dot{V}_{\text {stern }}(t)=Q_{\text {stern }} \quad \dot{V}_{\text {bow }}(t)=Q_{\text {bow }},
$$

mientras que la variación de la masa en los tanques es

$$
\Delta m_{\{\text {stern, bow }\}}(t)=\rho\left(V_{\{\text {stern, bow }\}}(t)-V_{0,\{\text { stern,bow }\}}\right) .
$$

El máximo caudal proporcionados por las bombas se ha supuesto de $\pm 20 \mathrm{l} / \mathrm{s}$ para cada una de ellas, mientras que para la máxima velocidad de cambio se ha empleado un valor de $\pm 5 \mathrm{l} / \mathrm{s}^{2}$. Estas cantidades son consistentes con las empleadas en [YH10, YJ10]. El tamaño y posición de los tanques es el mismo considerado con anterioridad para el sistema basado en soplado y ventilación.

\subsection{Simulaciones numéricas}

Nuestro propósito en esta sección es poner a prueba los sistemas basados en soplado/ventilación y bombeo frente a perturbaciones como las tratadas en el Capítulo 2. En la Sección 9.3.1 el vehículo estará sometido a cambios bruscos en el gradiente de densidad, mientras que en la Sección 9.3.2 consideraremos el efecto de las fuerzas producidas por un estado de mar 5 .

Para la primera de las simulaciones emplearemos el control óptimo como se describió en el Capítulo 5. Pese a que emplear una estrategia en lazo abierto, en la que supondremos que las perturbaciones son conocidas de antemano, puede en principio parecer poco realista, esto nos va a permitir hacer un primer análisis empleando las bien contrastadas herramientas matemáticas tratadas en el Capítulo 5. De esta forma los resultados serán independientes de la 
elección de un algoritmo de control, que podría resultar más adecuado para las características de alguno de los mecanismos de control propuestos.

La segunda de las simulaciones se llevará a cabo empleando el control en modo deslizante descrito en el Capítulo 7.

En ambos casos, el submarino estará inicialmente en vuelo estacionario a 4 nudos y en $t=0$ se detendrá la propulsión, de forma que el vehículo perderá velocidad de avance de forma progresiva (en ambos casos, la velocidad de avance al fin de la simulación es de alrededor de $0,4 \mathrm{kn})$.

\subsubsection{Escenario 9.1: Cambios bruscos de densidad. Control óptimo}

Sea $\mathbf{x} \in \mathbb{R}^{n}$ el vector de estado dado por (9.1) ó (9.2) según consideremos los sistemas de soplado/ventilación o bombeo respectivamente. Sea $\mathbf{u}$ el vector de control

$$
\mathbf{u}=\left[s_{\text {stern }}, s_{\text {bow }}, \bar{s}_{\text {stern }}, \bar{s}_{\text {bow }}\right] \quad \text { ó } \mathbf{u}=\left[Q_{\text {stern }}, Q_{\text {bow }}\right],
$$

y sea $\mathbf{A}(\mathbf{x}) \dot{\mathbf{x}}=\mathbf{f}(t, \mathbf{x}, \mathbf{u})$ la ley de estado compuesta de las ecuaciones cinemáticas y dinámicas más un conjunto de ecuaciones (3.19)-(3.21) por cada uno de los dos tanques en el caso del sistema basado en soplado/ventilación o las ecuaciones cinemáticas y dinámicas más las ecuaciones (9.3) en el caso del sistema basado en bombeo. El problema de control óptimo puede formularse como

$$
\begin{cases}\text { Minimizar en } \mathbf{u}: & J(\mathbf{u})=\Phi\left(\mathbf{x}\left(t_{f}\right), \mathbf{x}^{t_{f}}\right)+\int_{0}^{t_{f}} F(t, \mathbf{x}(t)) d t \\ \text { sujeto a } & \\ & \mathbf{A}(\mathbf{x}(t)) \dot{\mathbf{x}}(t)=\mathbf{f}(t, \mathbf{x}(t), \mathbf{u}(t)) \\ & \mathbf{x}(0)=\mathbf{x}^{0} \in \Omega \\ & \mathbf{x}(t) \in \Omega, \quad \mathbf{u}(t) \in \mathcal{K},\end{cases}
$$

donde $\Omega$ y $\mathcal{K}$ son los conjuntos en los que toman valores las variables de estado y control respectivamente y $\Phi\left(\mathbf{x}\left(t_{f}\right), \mathbf{x}^{t_{f}}\right), F(t, \mathbf{x}(t))$ están dados por (5.1), (5.2). En este caso deseamos mantener una cota de $50 \mathrm{~m}$ y el pitch alrededor de $0^{\circ}$. Para ello, tomaremos $z^{t_{f}}=z^{d}=50$, $\theta^{t_{f}}=\theta^{d}=0, \alpha_{3}=\beta_{3}=2, \alpha_{5}=\beta_{5}=1$. Véase el Capítulo 5 para una descripción completa de esta formulación.

En esta simulación el sistema de hovering ha de enfrentarse a bruscos cambios en la temperatura del agua, tal y como se muestra en la Figura 9.1. Entre $t=0$ y $t=500 \mathrm{~s}$ la temperatura del agua se considera constante. En $t=500 \mathrm{~s}$ el vehículo entra en una zona con un gradiente de temperatura de $-0,05{ }^{\circ} \mathrm{C} / \mathrm{m}$. En $t=1000 \mathrm{~s}$ el gradiente cambia a 0,05 ${ }^{\circ} \mathrm{C} / \mathrm{m} \mathrm{y}$, por último, en $t=1500 \mathrm{~s}$ se regresa al gradiente anterior. La dependencia de la flotabilidad del vehículo con la densidad del agua, y por tanto con la temperatura, se trató en la Sección 2.2.2. 


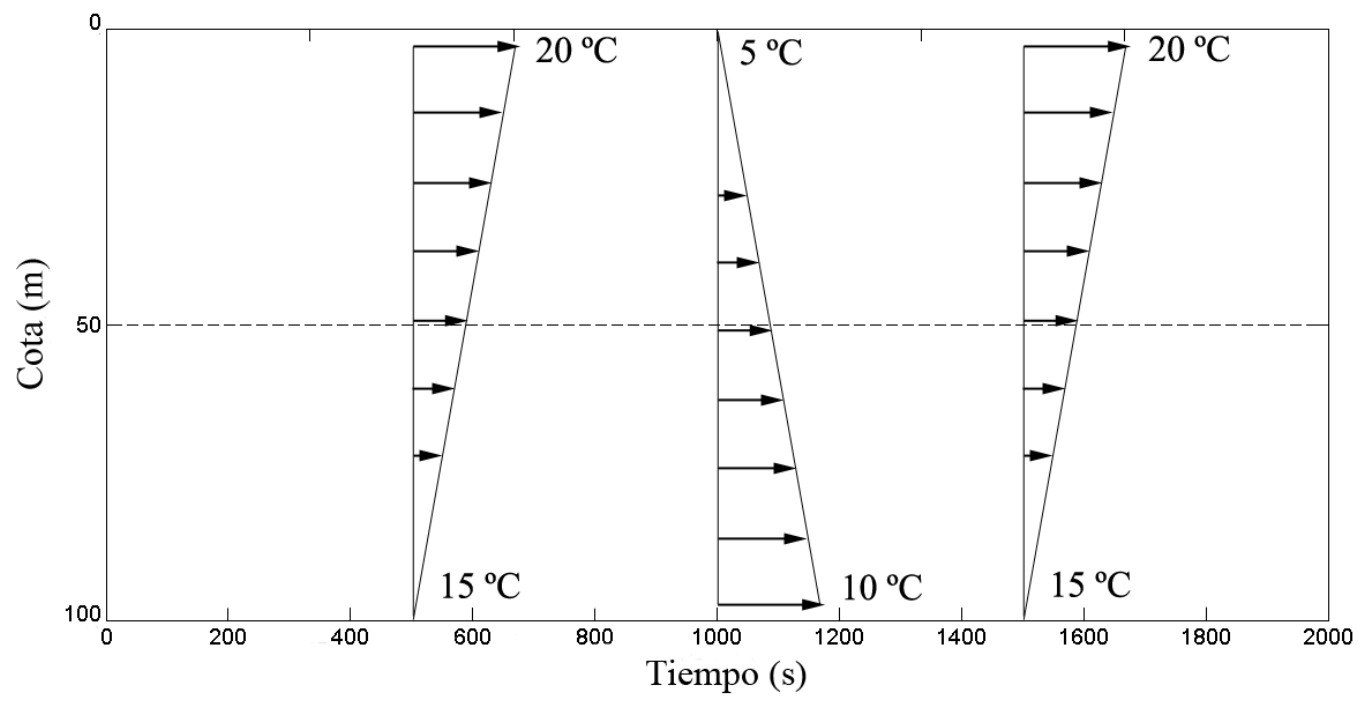

Figura 9.1: Gradiente de temperatura para el Escenario 9.1.

Las Figuras 9.2 y 9.3 muestran los resultados de esta primera simulación. En la Figura 9.2 puede verse la evolución de la cota obtenida empleando los sistemas basados en soplado y ventilación (línea roja) y bombeo (línea azul). La Figura 9.3 muestra el volumen de agua en los tanques (tanque de popa, izquierda y tanque de proa, derecha), el caudal bombeado por el sistema basado en bombas hidráulicas y la apertura de las válvulas de soplado y ventilación. Como puede verse en la Figura 9.2, las variaciones de cota obtenidas mediante el sistema basado en soplado y ventilación son notablemente menores que las que se obtienen empleando el sistema basado en bombeo. La clave parece ser (Figura 9.3, fila superior) la mayor velocidad de respuesta del sistema basado en soplado y ventilación. Además, la Figura 9.3 muestra que el uso del soplado/ventilación no sólo logra un mejor comportamiento, sino que éste es obtenido con una muy pequeña acción de control. 


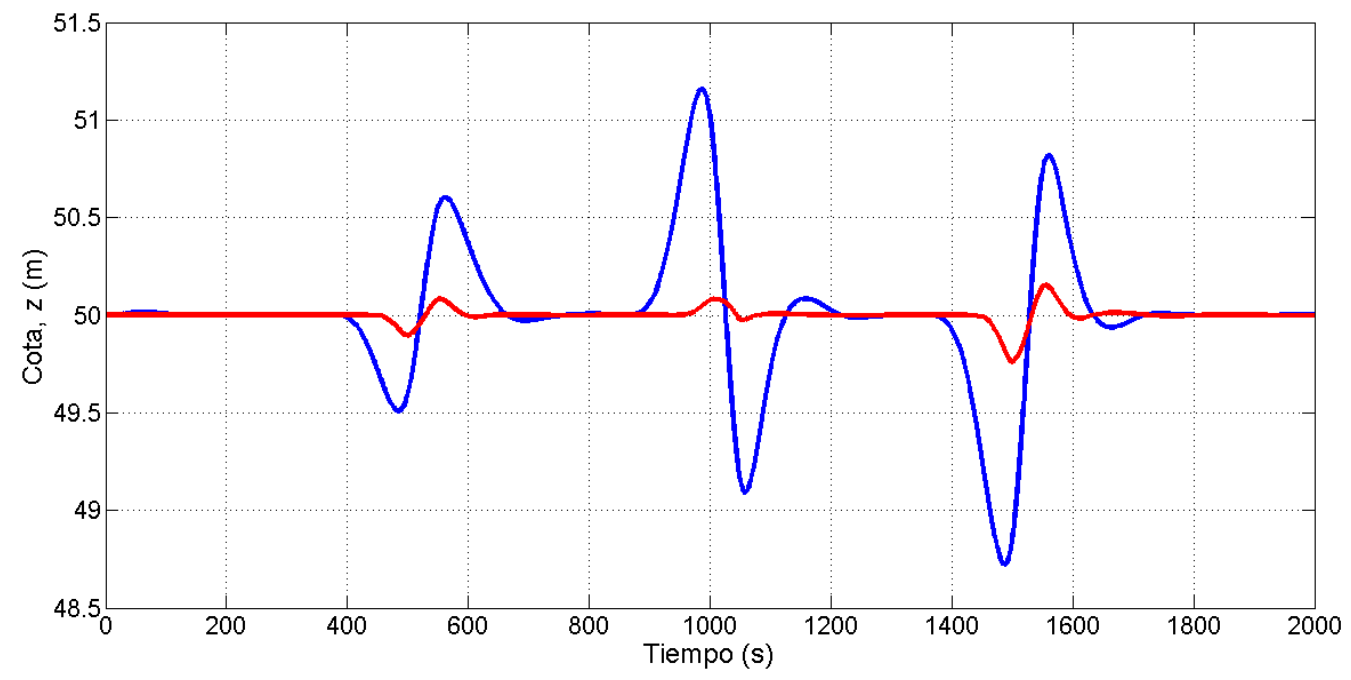

Figura 9.2: Comparación de la evolución de la cota para el Escenario 9.1 empleando control mediante bombeo (línea azul) y soplado/ventilación (línea roja). 

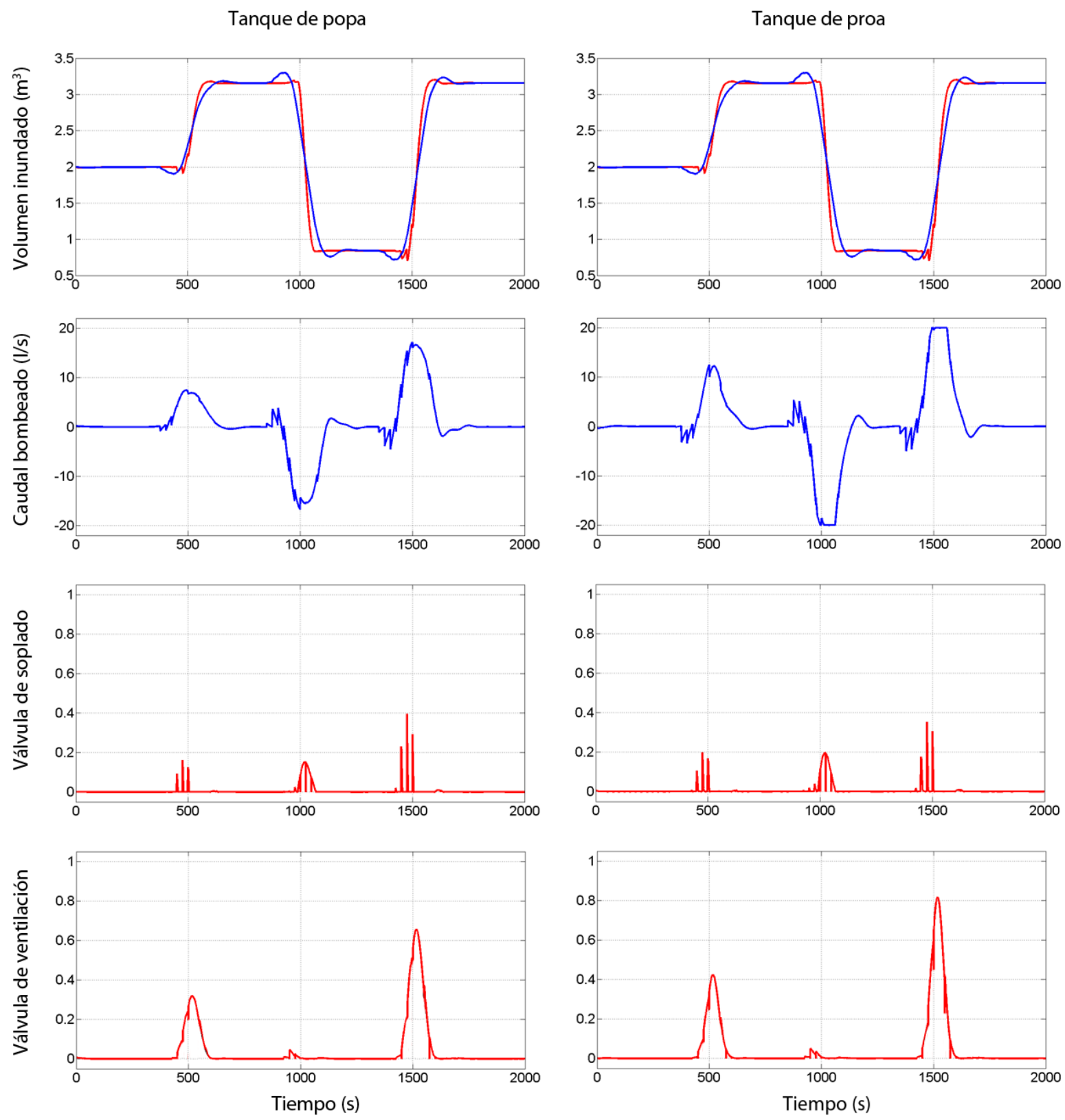

Figura 9.3: De arriba a abajo: volumen inundado en los tanques (tanque de popa, columna izquierda, tanque de proa, derecha), caudal bombeado y aperturas de las válvulas de soplado y ventilación. Las variables relativas al sistema basado en bombeo se muestran en azul mientras que las correspondientes al sistema basado en soplado/ventilación se muestran en rojo. 


\subsubsection{Escenario 9.2: Esfuerzos producidos por el oleaje. Control en modo deslizante}

En este caso el submarino se encuentra a $35 \mathrm{~m}$ de profundidad bajo la acción del oleaje correspondiente a un estado de mar 5 (véase la Sección 2.1). Emplearemos el esquema de control en modo deslizante descrito en el Capítulo 7 tanto para el sistema basado en soplado y ventilación como para el basado en bombeo. En el caso de éste último, es inmediato ver que se encuentra directamente en la forma afín (7.16). El grado relativo de este sistema es, al igual que en caso de emplearse soplado/ventilación, $r=3$. El diseño del controlador para el sistema basado en bombeo es por tanto completamente análogo al descrito en la Sección 7.3.

Recordemos que la ley de control propuesta es de la forma

$$
\mathbf{u}=\widehat{\mathbf{G}}^{*}(\mathbf{x})\left(-2 \lambda \ddot{\tilde{y}}-\lambda^{2} \dot{\tilde{y}}-k \operatorname{sat}(s / \Phi)-\widehat{F}(\mathbf{x})\right),
$$

con $k>\Delta$.

Una cota superior de $\Delta$ para un estado de mar 5 puede estimarse simulando el valor de los esfuerzos producidos por éste durante un intervalo de tiempo suficientemente largo. Mediante esta metodología, se ha estimado que la elección de $k=0,001$ parece ser suficiente para hacer frente tanto a cambios en la densidad del agua como a los esfuerzos producidos por un estado de mar 5. En cuanto al resto de parámetros (ver Sección 7.2) se ha tomado $\lambda=0,05, \Phi=0,001$.

La Figura 9.4 muestra la evolución de la cota empleando el sistema de bombeo (línea azul) y de soplado/ventilación (línea roja). Puede verse cómo, de nuevo, el sistema basado en soplado y ventilación proporciona resultados considerablemente más precisos.

Las variables de control durante un intervalo de 250 segundos se muestran en la Figura 9.5. En todos los casos, no se aprecia la presencia de chattering y las oscilaciones son suaves y compatibles con las restricciones físiscas consideradas.

En cuanto a la autonomía de las botellas de aire, en esta situación es de alrededor de 30 minutos y está por encima de los 90 minutos para la misma profundidad y estado de mar 4 .

\subsection{Conclusiones}

Se ha investigado la capacidad de un sistema de hovering basado en el soplado y ventilación de tanques de lastre y se ha contrastado su rendimiento con el obtenido empleando bombas hidráulicas. Los resultados de simulación muestran que los resultados obtenidos mediante soplado y ventilación pueden ser notablemente superiores.

El soplado y ventilación de tanques, en solitario o combinado con otros mecanismos de control, se perfila, por tanto, como un muy prometedor sistema de control para su integración en submarinos tripulados, AUVs u otros dispositivos submarinos. Este sistema no 


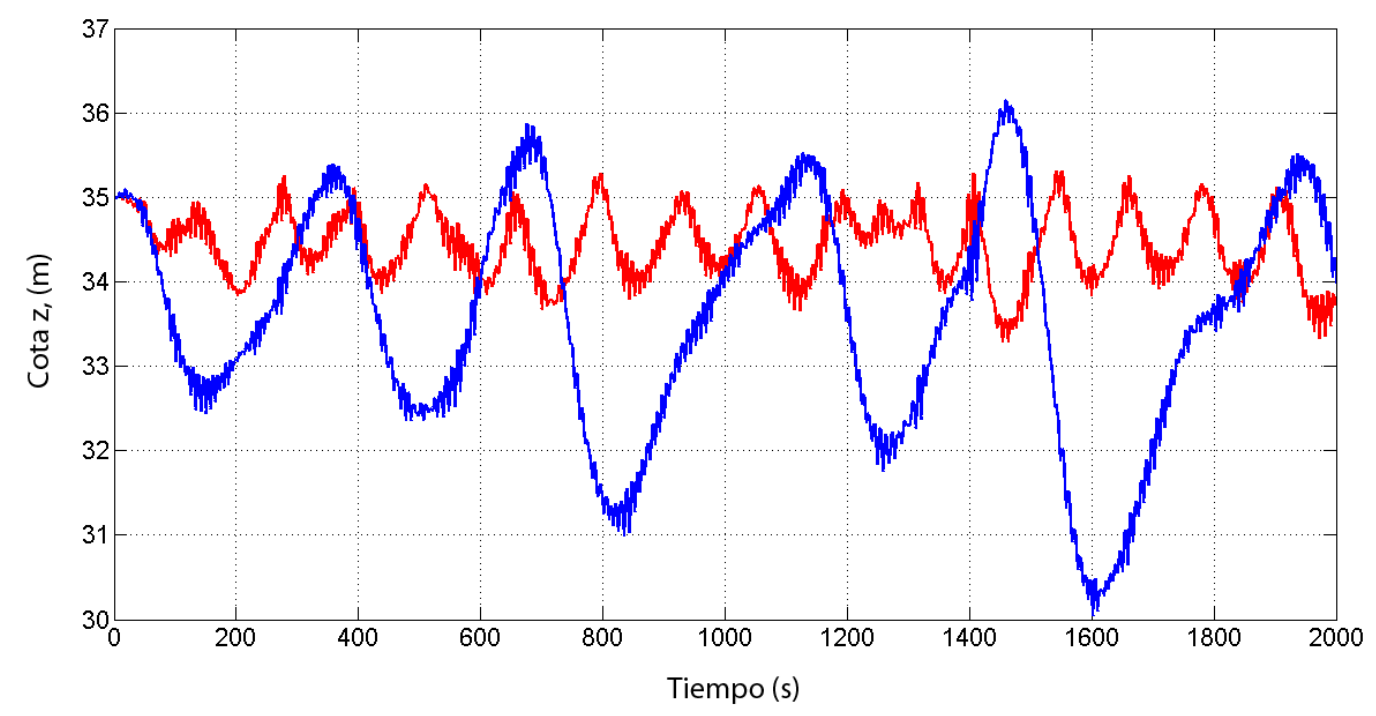

Figura 9.4: Comparación de la evolución de la cota para el Escenario 9.2 empleando control mediante bombeo (línea azul) y soplado/ventilación (línea roja).

sólo muestra un excelente rendimiento, sino que la energía necesaria para accionar las válvulas de soplado y ventilación debería ser notablemente inferior a la energía consumida por otros sistemas como bombas hidráulicas o propulsores.

$\mathrm{Su}$ alto rendimiento y su reducido consumo energético son, por tanto, las principales ventajas de este mecanismo de control. Por otro lado, la dependencia de botellas de aire implica una autonomía limitada y un aumento de peso, lo que constituye la principal desventaja en el caso de vehículos de pequeño tamaño como AUVs. Respecto a los submarinos tripulados, la principal limitación la constituye el ruido generado durante el proceso de soplado, muy elevado en el caso de los soplados de emergencia. En el caso del sistema de hovering los caudales son muy inferiores a los empleados al soplar los tanques de lastre principales, por lo que es posible que este problema pudiera mitigarse con un adecuado diseño del sistema de soplado. Ésta sería una importante cuestión a abordar en caso de desearse avanzar hacia la incorporación de este tipo de sistemas en vehículos reales. 

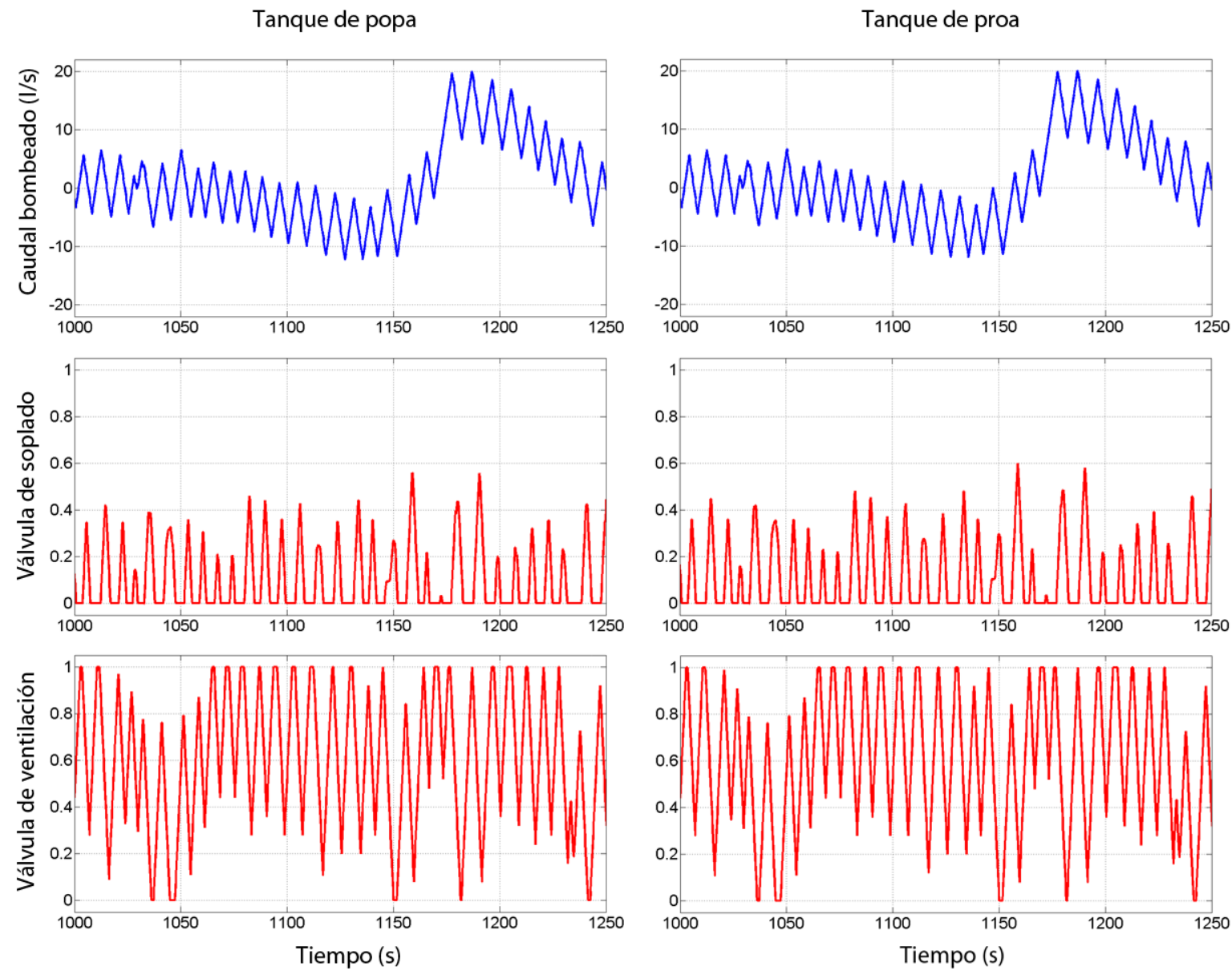

Figura 9.5: Caudal bombeado y apertura de las válvulas de soplado y ventilación del tanque de popa (izquierda) y proa (derecha). Las variables relativas al sistema basado en bombeo se muestran en azul mientras que las correspondientes al sistema basado en soplado/ventilación se muestran en rojo. 



\section{Conclusiones}

Se ha propuesto un modelo matemático para los procesos de soplado y ventilación de tanques de lastre acoplado, mediante las variaciones de masa producidas por estos procesos, con las ecuaciones que modelan el comportamiento dinámico del vehículo. Se ha llevado a cabo, así mismo, un riguroso análisis matemático del modelo resultante.

Se ha formulado el problema de control del vehículo mediante el soplado y ventilación de lastres y propuesto algoritmos de control en lazo abierto (incluyendo un teorema de existencia de solución del problema de control óptimo) y cerrado, tanto para el seguimiento de las trayectorias óptimas generadas en lazo abierto como para la estabilización ante perturbaciones de gran tamaño.

La suma de estos modelos y algoritmos de control, junto con los anteriormente desarrollados en [Ova11], constituye, hasta donde sabemos, el más completo conjunto de herramientas para el estudio de la maniobrabilidad de submarinos tripulados disponible actualmente en la literatura. Como parte del contrato suscrito entre la Universidad Politécnica de Cartagena y la empresa Navantia, hemos desarrollado una herramienta de simulación basada en Matlab llamada SimuSUB. Esperamos que esta herramienta, que integra todos los modelos y algoritmos desarrollados a lo largo de este trabajo, sea empleada en el futuro para poner a prueba la maniobrabilidad de nuevos diseños.

Como un segundo objetivo, hemos explorado el posible uso de los procesos de soplado y ventilación como un mecanismo de control complementario a hélice y timones que permita mejorar la maniobrabilidad del vehículo en determinado tipo de situaciones. Se ha explorado esta posibilidad en dos contextos diferenciados:

a) Se ha demostrado, a nivel de simulación numérica, que el soplado diferencial de los tanques de lastre, incorporando la apertura variable de las válvulas de soplado, puede mejorar sustantivamente la estabilidad y seguridad durante soplados de emergencia con respecto a las secuencias preprogramadas con aperturas todo o nada empleadas en la actualidad. 
b) Se ha mostrado, así mismo, cómo un sistema basado en el soplado y ventilación de un conjunto de tanques dispuestos al efecto permite realizar el hovering manteniendo la cota en unas tolerancias considerablemente más estrechas que las logradas por sistemas basados en el uso de bombas hidráulicas.

Estos resultados, aunque prometedores, constituyen sin embargo sólo un primer paso. Puesto que los tanques de lastre no son, por su tamaño, adecuados para el control de la flotabilidad necesario para el hovering, esta segunda aproximación requiere el diseño de unos tanques y sistemas de soplado/ventilación específicos para esta tarea. El empleo de un sistema de control que permita el soplado diferencial de los tanques de lastre, por el contrario, podría ser incorporado en diseños existentes con mínimas modificaciones. Antes de ello, y puesto que los trabajos realizados hasta el momento constituyen únicamente un estudio preliminar, es necesario llevar a cabo las tareas que garanticen la viabilidad de su implementación en un vehículo real:

- Validación experimental de los modelos matemáticos para el soplado y ventilación de lastres. La precisión de estos modelos es esencial para el posterior diseño de sistemas de control. En el Capítulo 4 pueden encontrarse recomendaciones sobre posibles áreas de mejora y cómo refinar el modelo en caso de que se estimara necesario.

- El control del soplado requiere válvulas de soplado que permitan una apertura variable. Es necesario estudiar las posibles válvulas a instalar e incorporar sus características particulares (tiempos de apertura, pérdidas locales de presión, etc.) al modelo.

- Estudio de las modificaciones necesarias en los sistemas de soplado: incorporación de sensores, actuadores, controladores, electrónica.

- Análisis, mediante un programa de ensayos, del rendimiento y mejoras ofrecidas por el nuevo sistema de soplado.

Por supuesto, la lista anterior no es exhaustiva y otras tareas podrían ser necesarias.

Una vez realizado este desarrollo, los resultados y conclusiones podrían fácilmente ser extrapolados al diseño de un sistema de hovering basado en soplado y ventilación de tanques.

Desde el punto de vista académico, existen algunas líneas abiertas, como probar la existencia de solución para el problema de control óptimo empleando timones y soplado/ventilación de forma simultánea o la demostración de la convergencia de la solución proporcionada por el esquema de control propuesto en el Capítulo 6 a la solución en lazo abierto a medida que el periodo de muestreo tiende a cero. El excelente comportamiento mostrado por el esquema de control en modo deslizante sugiere, por último, que ésta puede ser otra interesante línea de investigación futura. 


\section{Anexo. Ajuste experimental de los esfuerzos producidos por el oleaje}

Ángulo relativo $180^{\circ}$

Fuerza vertical

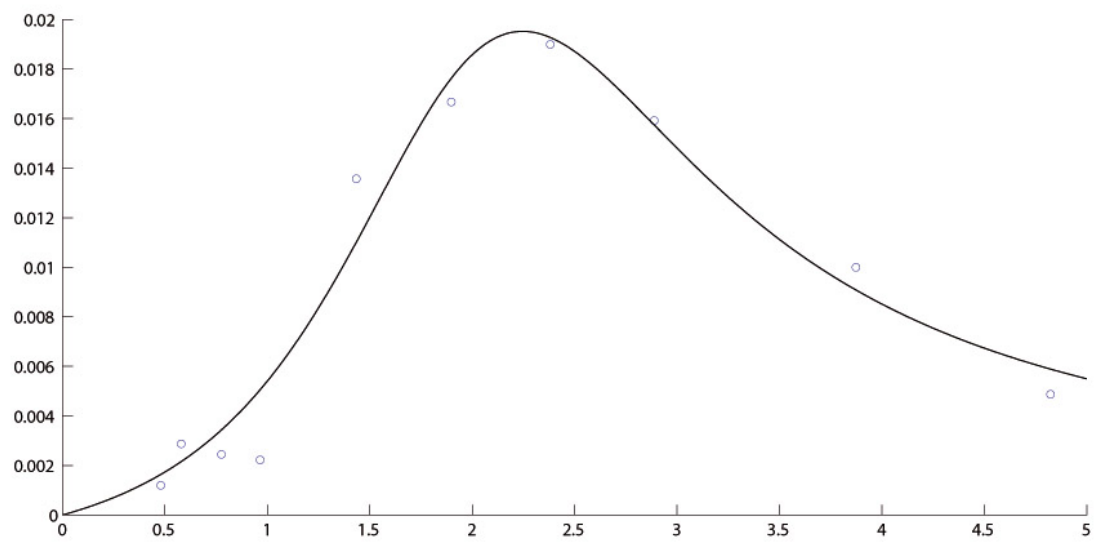

Figura 6: Fuerza vertical. Amplitud (180).

$$
\frac{0,01161 \lambda}{\lambda^{2}-3,898 \lambda+5,046}
$$




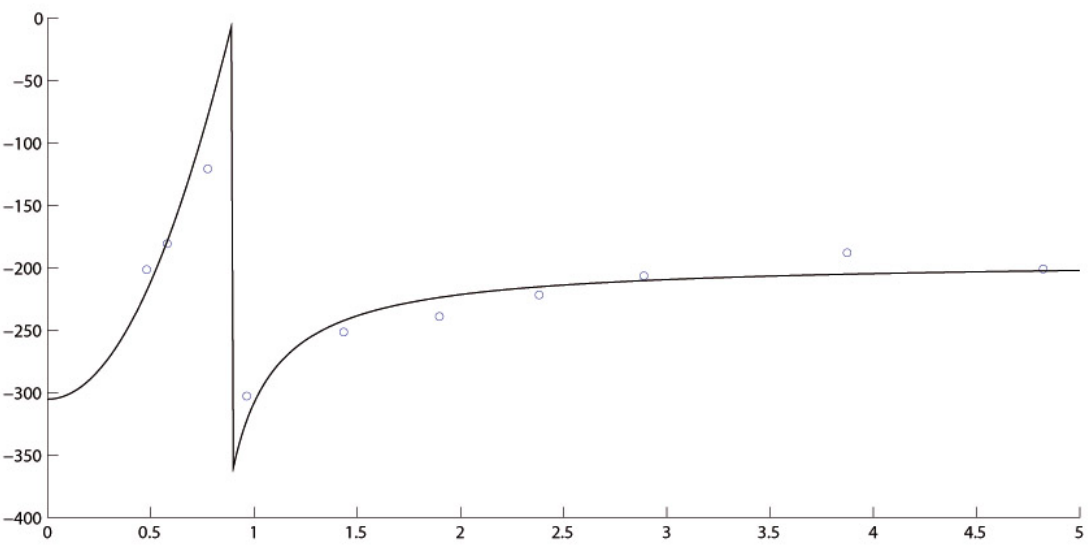

Figura 7: Fuerza vertical. Desfase $\left(180^{\circ}\right)$.

$$
\left\{\begin{array}{r}
376,5 \lambda^{2}-305,3 \\
\text { if } \quad \lambda \leq 0,9 \\
-193,7 \lambda+94,65 \\
\hline \lambda-0,6787 \\
\text { if } \quad \lambda>0,9
\end{array}\right.
$$




\section{Momento de giro}

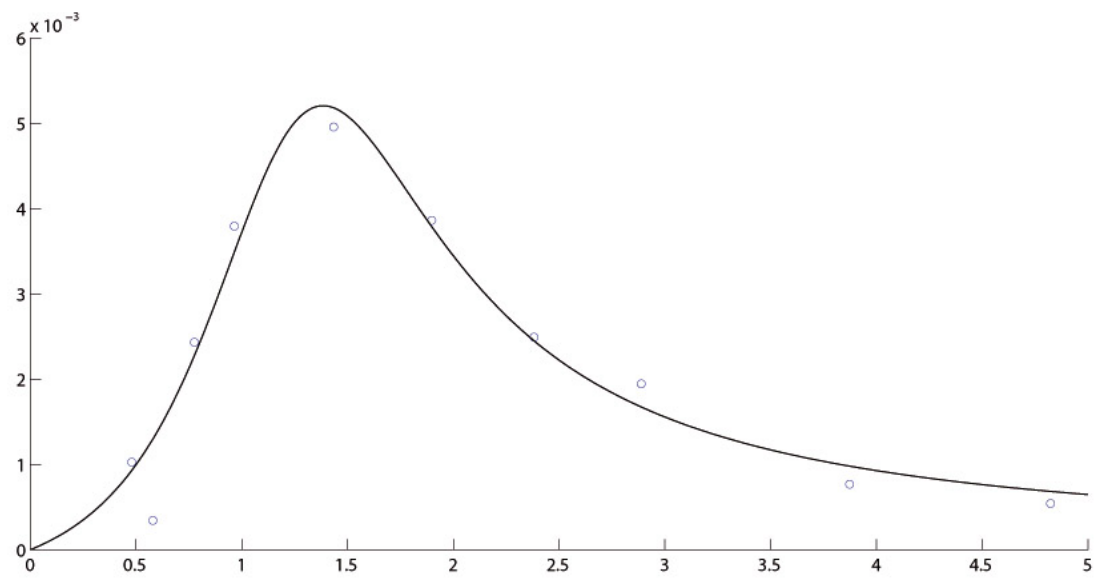

Figura 8: Momento de giro. Amplitud $\left(180^{\circ}\right)$.

$$
\frac{0,001932 \lambda}{\lambda^{2}-2,398 \lambda+1,917}
$$

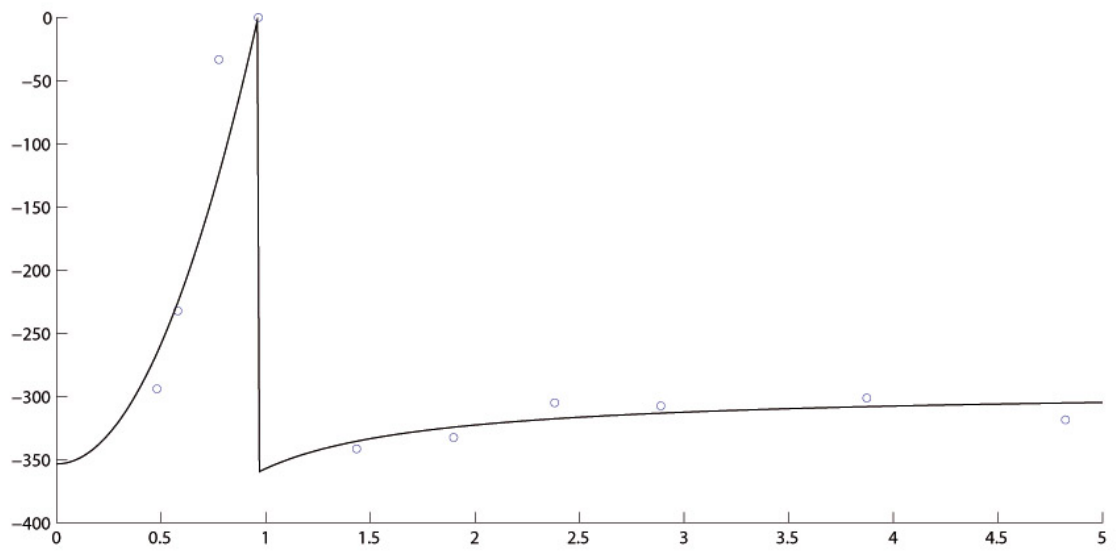

Figura 9: Momento de giro. Desfase $\left(180^{\circ}\right)$.

$$
\left\{\begin{array}{c}
380,9 \lambda^{2}-353,5 \\
\text { if } \quad \lambda \leq 0,964 \\
\frac{-293,9 \lambda-4,822}{\lambda-0,1637} \\
\text { if } \quad \lambda>0,964
\end{array}\right.
$$




\section{Ángulo relativo $135^{\circ}$}

\section{Fuerza vertical}

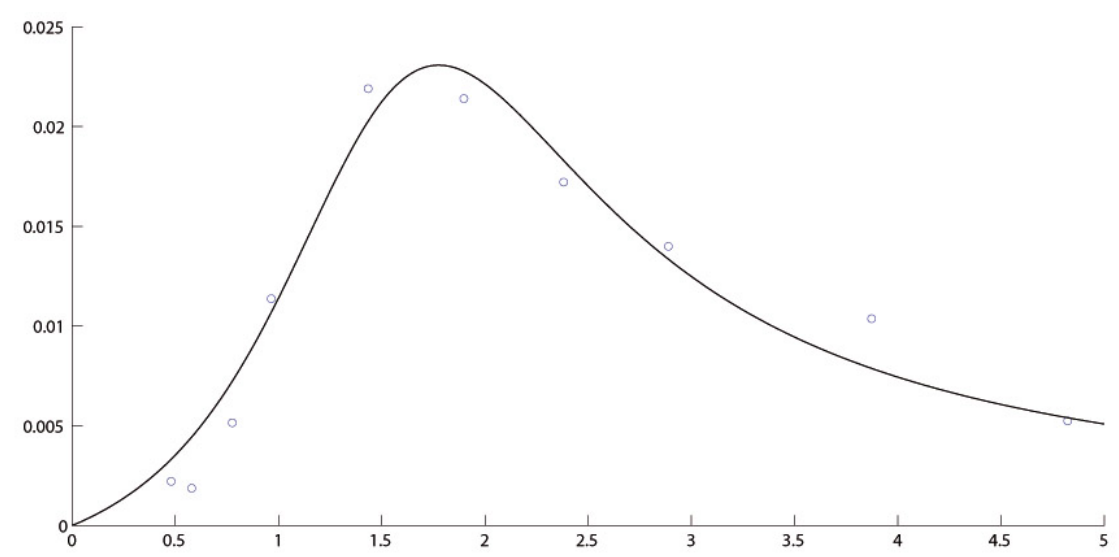

Figura 10: Fuerza vertical. Amplitud $\left(135^{\circ}\right)$.

$$
\frac{0,01358 \lambda}{\lambda^{2}-2,964 \lambda+3,155}
$$

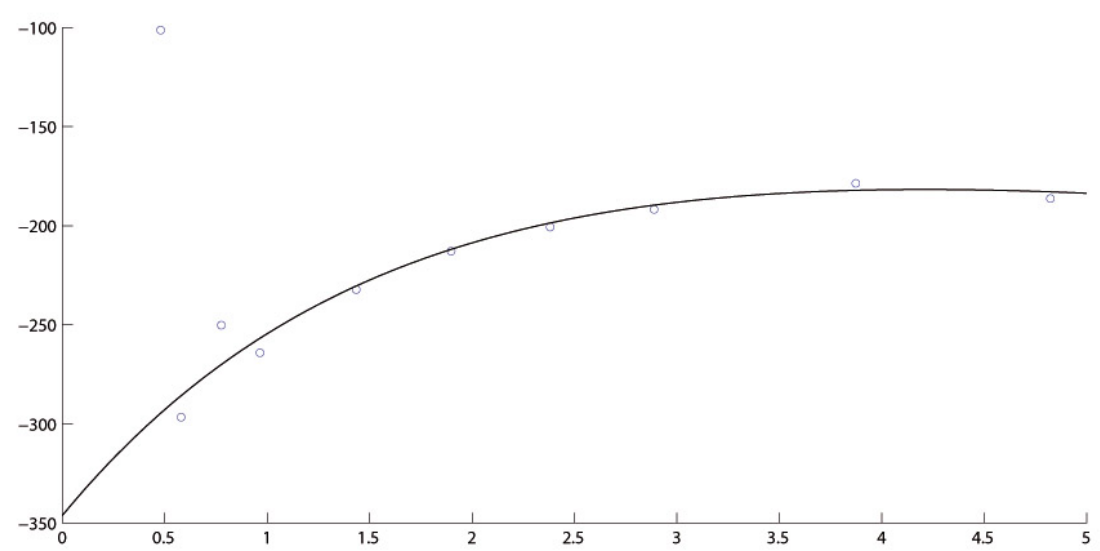

Figura 11: Fuerza vertical. Desfase $\left(135^{\circ}\right)$.

$$
-220,4 \cdot \mathrm{e}^{(-0,6043 \lambda)}-125,8 \cdot \mathrm{e}^{0,06538 \lambda}
$$




\section{Momento de giro}

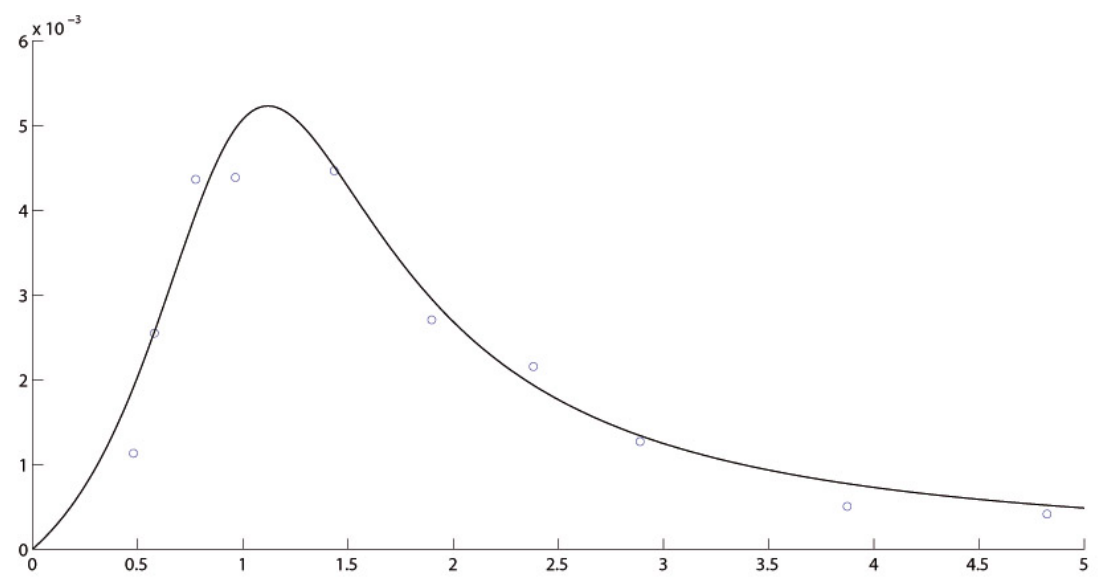

Figura 12: Momento de giro. Amplitud $\left(135^{\circ}\right)$.

$$
\frac{0,01967 \lambda}{\lambda^{3}+4,95 \lambda^{2}-11,08 \lambda+9,008}
$$

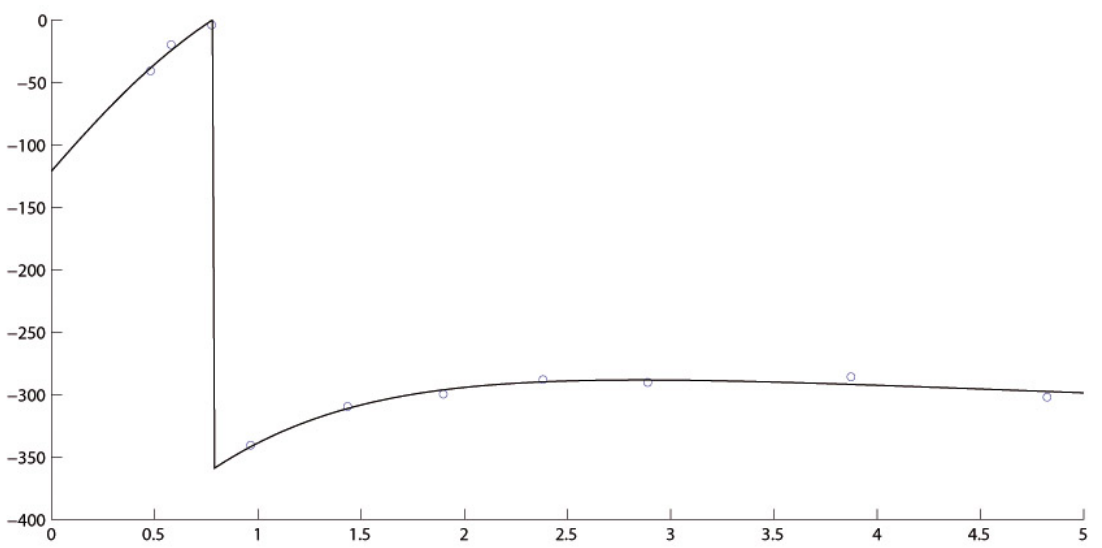

Figura 13: Momento de giro. Desfase $\left(135^{\circ}\right)$.

$$
\frac{468,6 \lambda-365,6}{\lambda^{2}+0,84 \lambda+3,021}
$$




\section{Ángulo relativo $90^{\circ}$}

\section{Fuerza vertical}

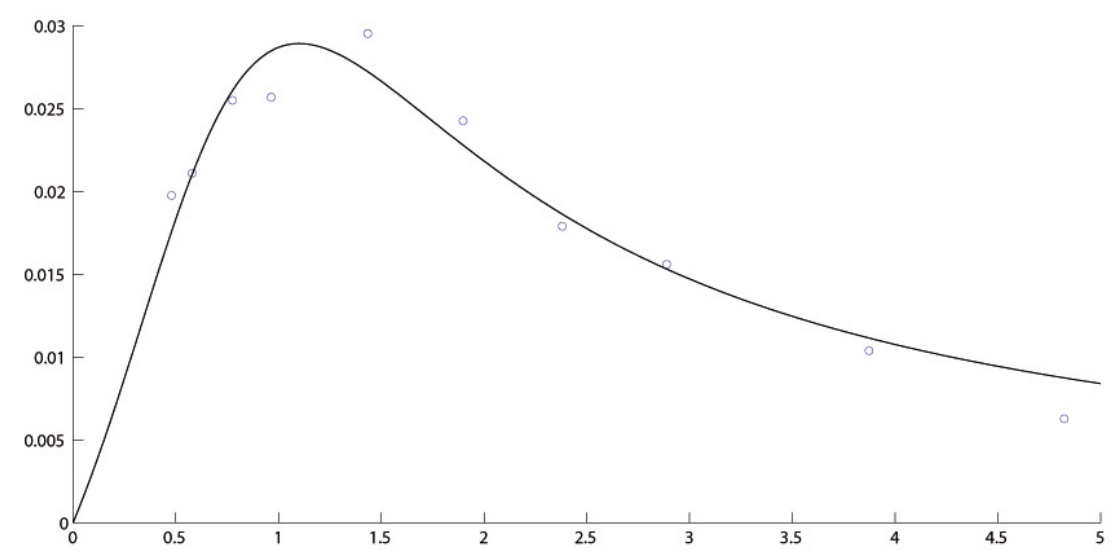

Figura 14: Fuerza vertical. Amplitud $\left(90^{\circ}\right)$.

$$
\frac{0,03601 \lambda}{\lambda^{2}-0,9567 \lambda+1,212}
$$

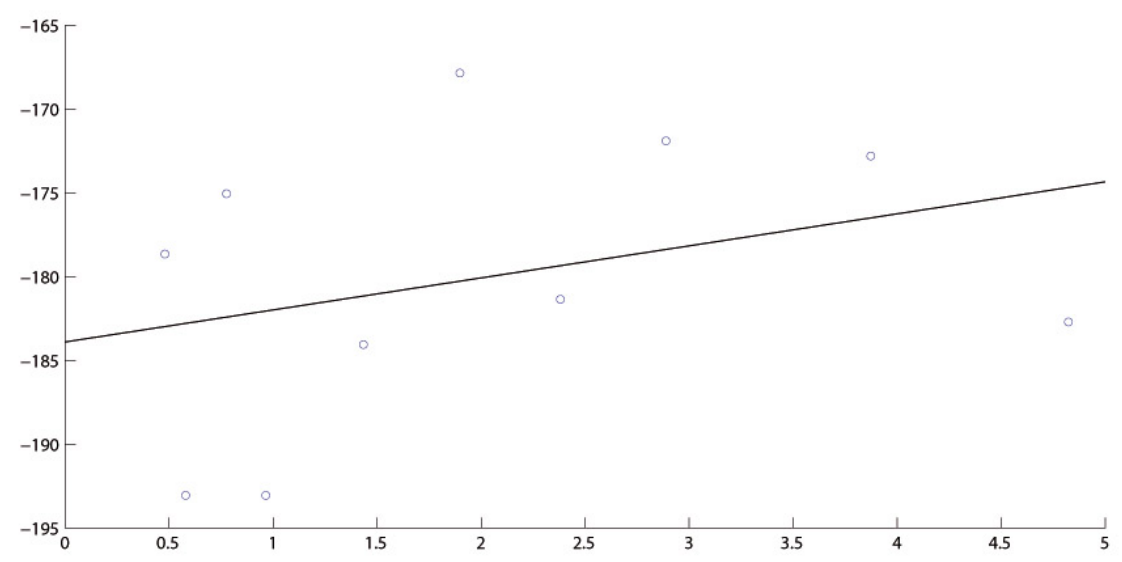

Figura 15: Fuerza vertical. Desfase $\left(90^{\circ}\right)$.

$$
1,911 \lambda-183,9
$$




\section{Momento de giro}

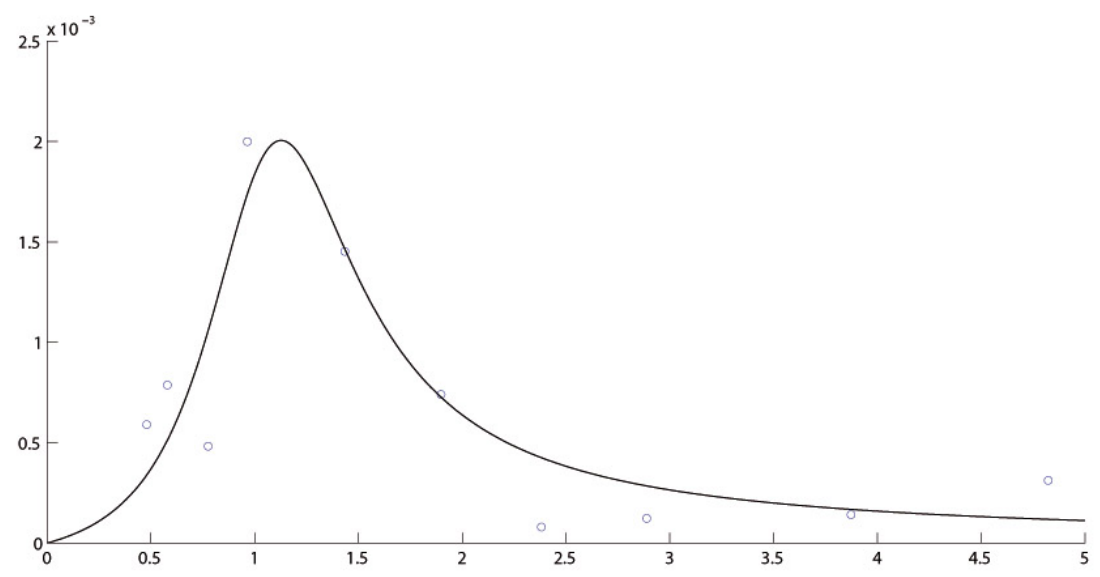

Figura 16: Momento de giro. Amplitud $\left(90^{\circ}\right)$.

$$
\frac{0,0003565 \lambda}{\lambda^{2}-2,077 \lambda+1,271}
$$

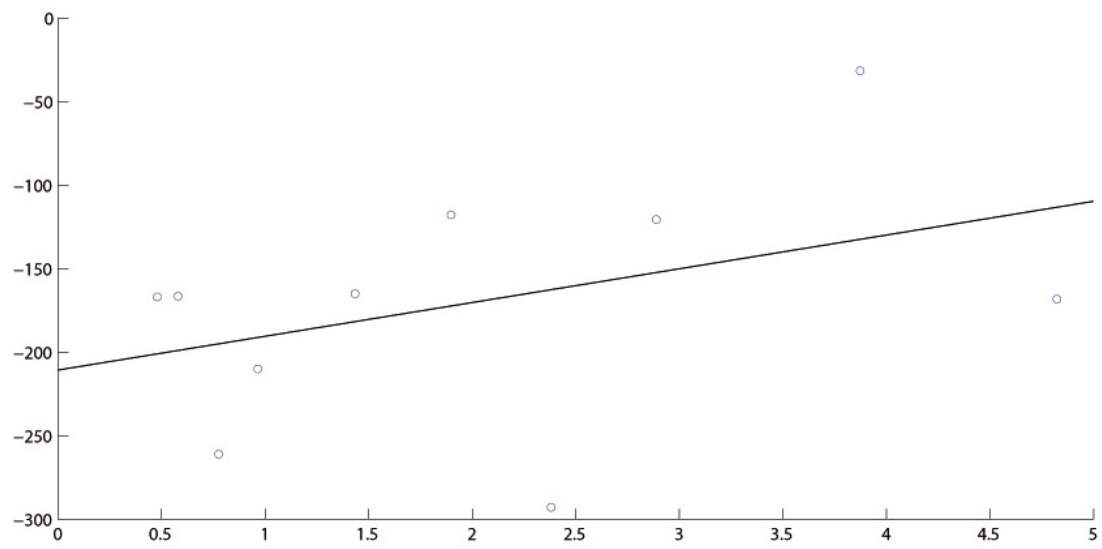

Figura 17: Momento de giro. Desfase $\left(90^{\circ}\right)$.

$$
20,2 \lambda-210,7
$$




\section{Ángulo relativo $45^{\circ}$}

\section{Fuerza vertical}

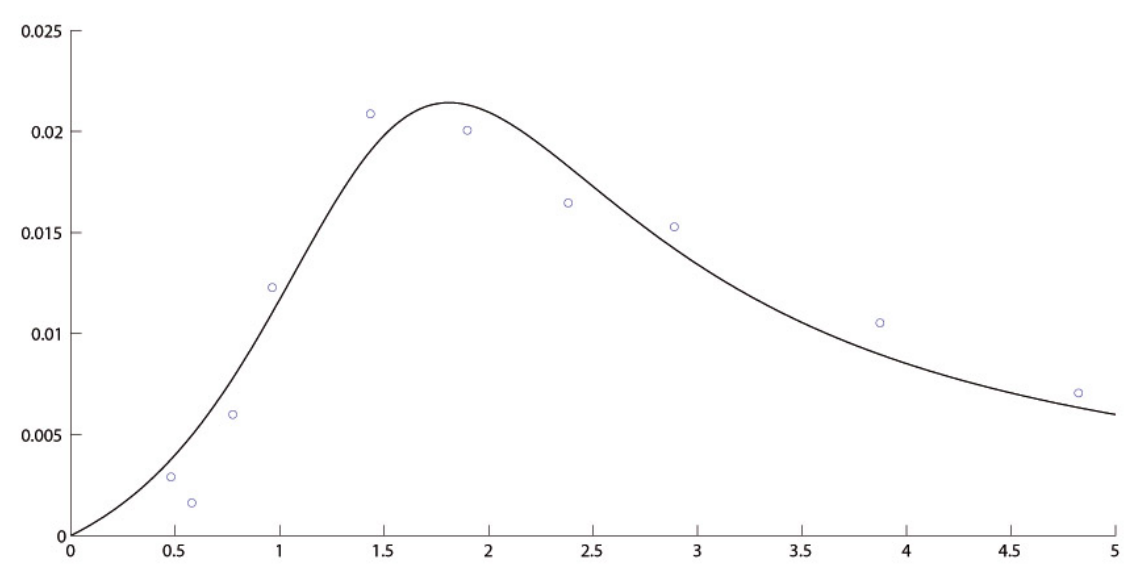

Figura 18: Fuerza vertical. Amplitud $\left(45^{\circ}\right)$.

$$
\frac{0,01692 \lambda}{\lambda^{2}-2,833 \lambda+3,281}
$$

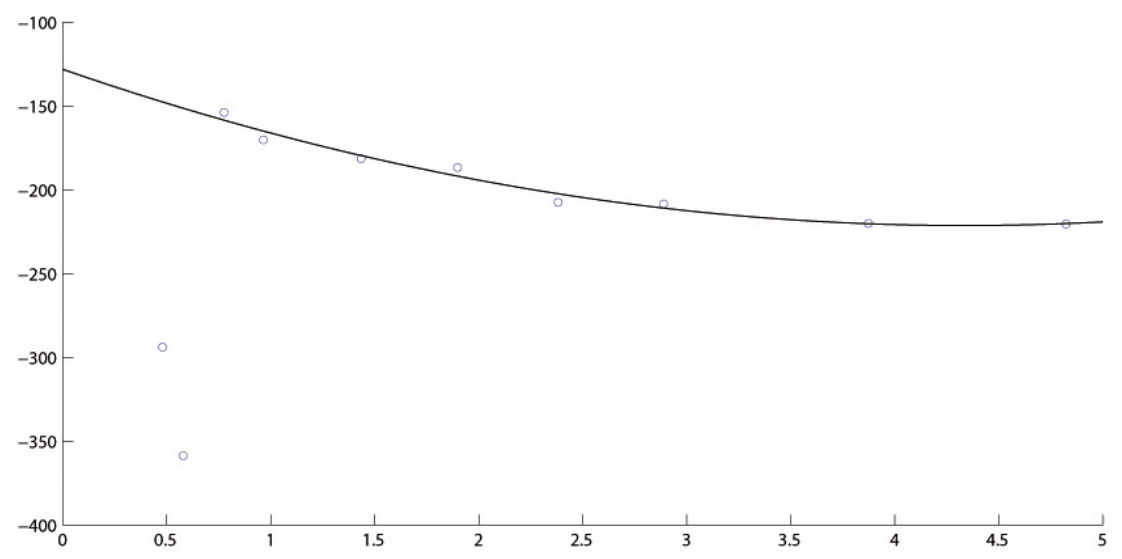

Figura 19: Fuerza vertical. Desfase $\left(45^{\circ}\right)$.

$$
4,954 \lambda^{2}-42,98 \lambda-128,1
$$




\section{Momento de giro}

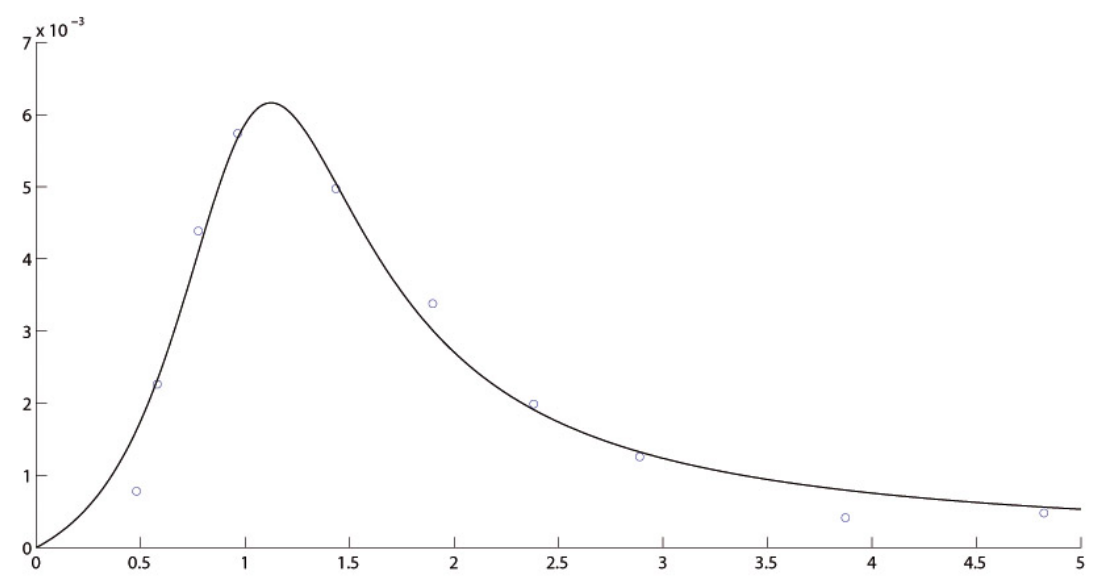

Figura 20: Momento de giro. Amplitud $\left(45^{\circ}\right)$.

$$
\frac{0,09266 \lambda}{\lambda^{3}+45,75 \lambda^{2}-91,62 \lambda+60,66}
$$

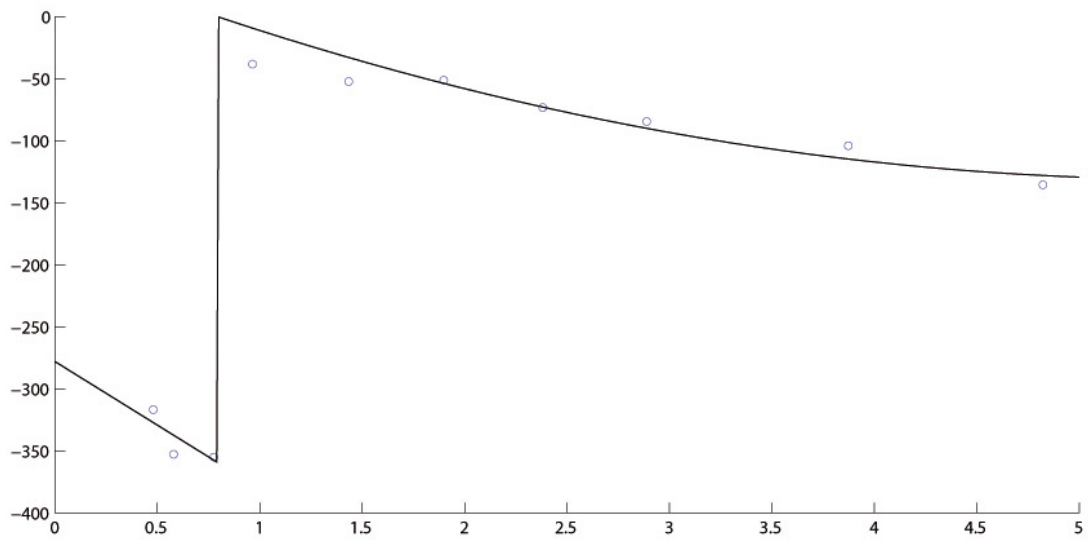

Figura 21: Momento de giro. Desfase $\left(45^{\circ}\right)$.

$$
\left\{\begin{array}{c}
-102,5 \lambda-278 \\
\text { if } \quad \lambda \leq 0,8 \\
5,784 \lambda^{2}-64,26 \lambda+47,33 \\
\text { if } \quad \lambda>0,8
\end{array}\right.
$$




\section{Ángulo relativo $0^{\circ}$}

\section{Fuerza vertical}

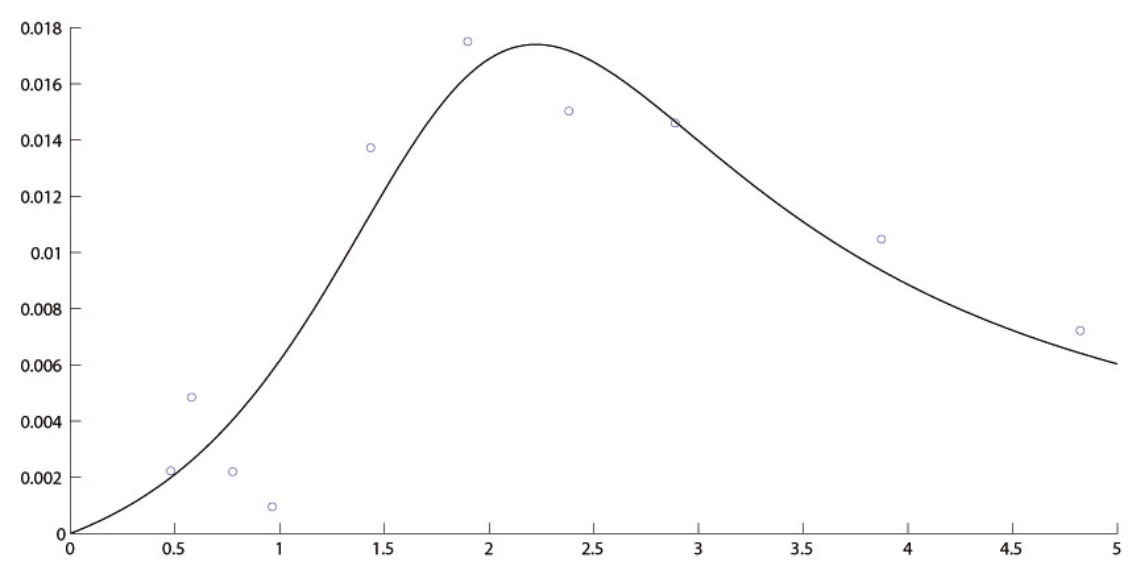

Figura 22: Fuerza vertical. Amplitud $\left(0^{\circ}\right)$.

$$
\frac{0,01424 \lambda}{\lambda^{2}-3,628 \lambda+4,943}
$$

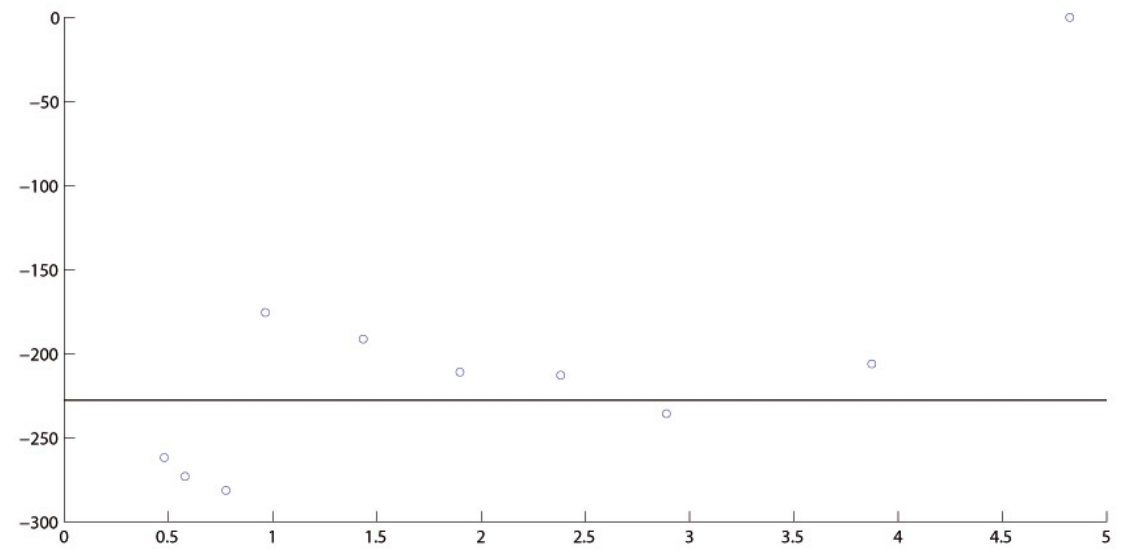

Figura 23: Fuerza vertical. Desfase $\left(0^{\circ}\right)$. 
Momento de giro

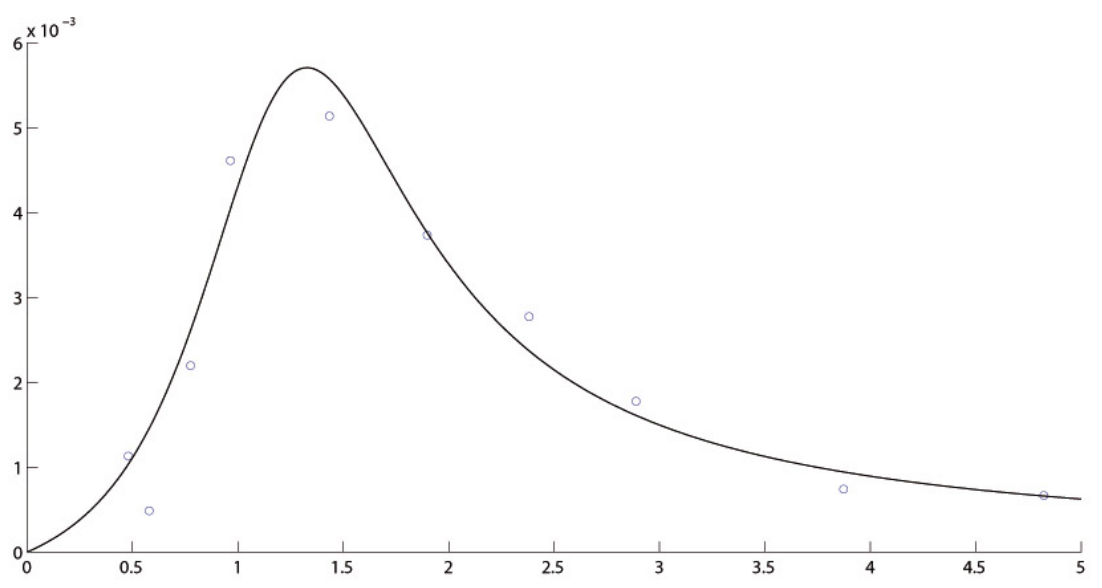

Figura 24: Momento de giro. Amplitud ( $\left.0^{\circ}\right)$.

$$
\frac{0,001892 \lambda}{\lambda^{2}-2,324 \lambda+1,763}
$$

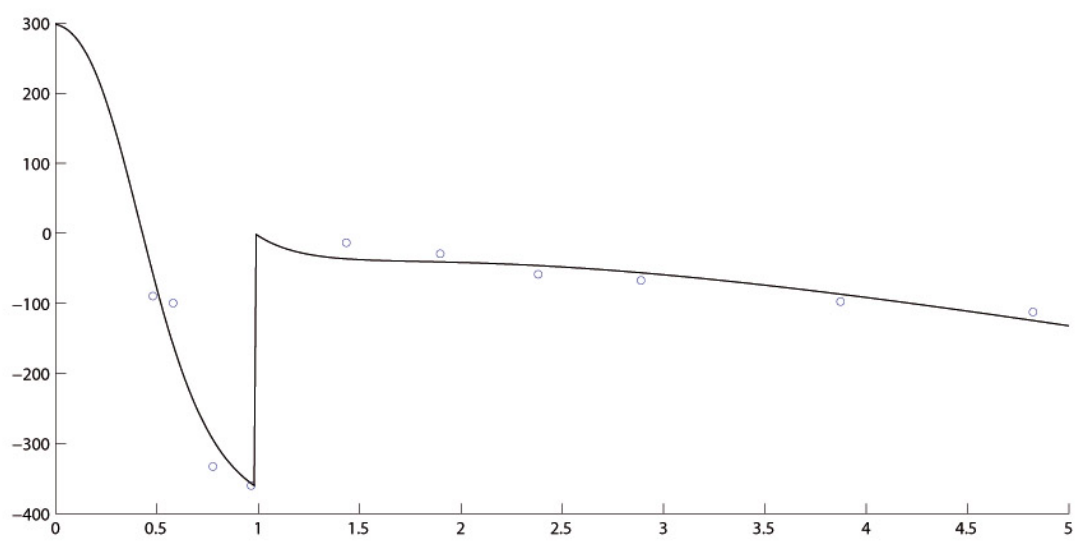

Figura 25: Momento de giro. Desfase $\left(0^{\circ}\right)$.

$$
\frac{-54,39 \lambda^{3}+233,6 \lambda^{2}-447 \lambda+265}{\lambda^{2}-0,6416 \lambda+0,4026}
$$





\section{Bibliografía}

[BGW09] M. C. Bettle, A. G. Gerber and G. D. Watt. Unsteady analysis of the six DOF motion of a buoyantly rising submarine. Computers and Fluids, 38 (2009), 1833-1849.

[Boo83] T. B. Bоотн. Optimal depth control of an underwater vehicle under a seaway. In RINA International Symposium on Naval Submarines. London, UK, 1983.

[Bys88] L. Byström. Adaptive control of a submarine in a snorting condition in waves. In Warship 88. London, UK, 1988.

[Bys01a] L. Byström. Submarine P-650. Captive manoeuvring tests and simulations. Report 2000 0101-4. SSPA Sweden, 2001.

[Bys01b] L. Byström. Submarine P-650. Depth Keeping when Snorting in Rough Seas. Report 2000 0101-12. SSPA Sweden, 2001.

[Bys04] L. Byström. Submarine recovery in case of flooding. Naval Forces, XXV (2004).

[Car11] J. CARlton. Marine propellers and propulsion, 3rd Edition. ButterworthHeinemann, 2011.

[CB04] E. F. CAmacho and C. Bordons. Control predictivo: presente, pasado y futuro. Revista Iberoamericana de Automática e Informática Industrial, 1 (2004), $5-28$.

[CB07] E. F. Camacho and C. Bordons. Model Predictive Control, 2nd Edition. Springer-Verlag, London, UK, 2007.

[Ces83] L. Cesari. Optimization theory and applications. Applications of Mathematics, 17. Springer-Verlag, New York Heidelberg Berlin, 1983.

[CHCY03] H. T. Choi, A. Hanai, S. K. Choi and J. Yuh. Development of an underwater robot, ODIN-III. In International conference on intelligent robots and systems. Las Vegas, USA, 2003. 
[Cul94] J. C. Culioli. Introduction à l'optimisation. Éditions Ellipses, Paris, 1994.

[DK04] U. Demirci and F. KerestecioĞLU. A re-configuring sliding-mode controller with adjustable robustness. Ocean Engineering, 31 (2004), 1669-1682.

[Fel79] J. Feldman. Revised standard submarine equations of motion. Report DTNSRDC/SPD-0393-09. David W. Taylor Naval Ship Research and Development Center, Washington DC, 1979.

[FF91] T. I. Fossen and B. A. Foss. Sliding control of MIMO nonlinear systems. In European Control Conference. Grenoble, France, 1991.

[FGO10] R. Font, J. García and D. Ovalle. Modeling and simulating ballast tank blowing and venting operations in manned submarines. In 8th IFAC Conference on Control Applications in Marine Systems. Rostock-Warnemünde, Germany, 2010.

[FGP12] R. Font and J. García-PelÁEz. On a submarine hovering system based on blowing and venting of ballast tanks. Submitted for publication, (2012).

[FGPMP12] R. Font, J. García-Peláez, J. A. Murillo and F. Periago. Modelling, analysis and control of blowing-venting operations in manned submarines. Submitted for publication, (2012).

[FM83] N. P. Fofonoff and R. C. Millard. Algorithms for computation of fundamental properties of seawater. UNESCO Technical papers in marine sciences, 44. 1983.

[Fos94] T. I. Fossen. Guidance and control of ocean vehicles. John Wiley \& sons, Washington DC, 1994.

[FPP] R. Font, P. Pedregal and F. Periago. A numerical method for computing optimal controls in feedback and digital forms and its application to the blowingventing control system of manned submarines. Accepted in Optimal Control Applications and Methods.

[GH67] M. Gertler and G. R. HAGEn. Standard equations of motion for submarine simulation. NSRDC Report 2510, 1967.

[GOP11] J. García, D. Ovalle and F. Periago. Analysis and numerical simulation of a nonlinear mathematical model for testing the manoeuvrability capabilities of a submarine. NonLinear Analysis B: Real World Applications, 12 (2011), 1654-1669. 
[HB08] T. HARDY and G. BARLOW. Unmanned underwater vehicle UUV deployment and retrieval considerations for submarines. In 9th International Naval Engineering Conference and Exhibition. Hamburg, Germany, 2008.

[Hic90] R. I. HICKEY. Submarine motion simulation including zero forward speed and propeller race effects. Master's thesis, Massachusetts Institute of Technology, 1990.

[HL93] A. J. Healey and D. Lienard. Multivariable sliding-mode control for autonomous diving and steering of unmanned vehicles. IEEE Journal of Oceanic Engineering, 18 (1993), 327-339.

[Isi95] A. IsIdori. Non Linear Control Systems, 3rd Edition. Communications and Control Engineering Series. Springer Verlag, London, UK, 1995.

[Kha01] H. K. KhaliL. Nonlinear Systems, 3rd Edition. Prentice Hall, New Jersey, USA, 2001.

[Kir04] D. E. KIRK. Optimal Control Theory: An Introduction. Dover Publications, New York, USA, 2004.

[LAm99] R. K. Lea, R. Allen and S. L. Merry. A comparative study of control techniques for an underwater flight vehicle. International Journal of Systems Science, 30 (1999), 947-964.

$\left[\mathrm{LPO}^{+} 11\right]$ J.-H. Li, S.-K. Park, S.-S. Oh, J.-H. Suh, G.-H. Yoon and M.-S. Beak. Development of a hovering-type intelligent autonomous underwater vehicle, $\mathrm{P}$ SURO. In Autonomous Underwater Vehicles, edited by N. A. CRUZ. InTech, Rijeka, Croatia, 2011.

[MCO11] E. A. Martínez-Conesa and D. OAKLey. Naval architecture challenges for integration of unmanned underwater vehicles in submarines. In 1st Submarine Institute of Australia Technology and Engineering Conference. Adelaide, Australia, 2011.

[Ova11] D. Ovalle. Nonlinear optimal control strategies for maneuverability analysis of manned submarines. Ph.D. thesis, Universidad Politécnica de Cartagena, Spain, 2011.

[P0́5] T. PÉrez. Ship Motion Control. Course Keeping and Roll Stabilisation Using Rudder and Fins. Springer-Verlag, London, UK, 2005.

[Ped03] P. Pedregal. On the generality of variational principles. Milan Journal of Mathematics, 71 (2003), 319-356. 
[PT10] F. Periago and J. Tiago. A local existence result for an optimal control problem modeling the manoeuvring of an underwater vehicle. Nonlinear Analysis: Real World Applications, 11 (2010), 2573-2583.

[PU11] A. PisAno and E. UsAI. Sliding mode control: A survey with applications in math. Mathematics and Computers in Simulation, 81 (2011), 954-979.

[RHMB05] J. Riedel, A. Healey, D. Marco and B. Beyazay. Design and development of low cost variable buoyancy system for the soft grounding of autonomous underwater vehicles. Naval Postgraduate School Center for AUV Research, Monterrey CA, 2005.

[SL91] J. J. Slotine and W. Li. Applied Nonlinear Control. Prentice Hall, Englewood Cliffs, New Jersey, 1991.

[SNA50] The Society of Naval Architects and Marine Engineers. Nomenclature for treating the motion of a submerged body through a fluid. Technical and Research Bulletin, (1950).

[Son90] E. Sontag. Mathematical control theory. Texts in Applied Mathematics, 6. Springer-Verlag, New York, USA, 1990.

[SR97] L. Shampine and M. Reichelt. The matlab ode suite. SIAM Journal on Scientific Computing, 18 (1997), 1-22.

[STF70] N. Salvesen, E. O. Tuck and O. Faltinsen. Ship motions and sea loads. Transactions of the Society of Naval Architects and Marine Engineers, 78 (1970), 250-287.

[Sva87] K. SvanBERG. The method of moving asymptotes - A new method for structural optimization. International journal for numerical methods in engineering, $\mathbf{2 4}$ (1987), 359-373.

[TD07] S. TANGirala and J. DzIELSKI. A variable buoyancy control system for a large AUV. IEEE Journal of Oceanic Engineering, 32 (2007), 762-771.

[VDD $\left.{ }^{+} 10\right]$ I. Vasilescu, C. Detweiler, M. Doniec, D. Gurdan, S. Sosnowski, J. StumpF and D. Rus. AMOUR V: A hovering energy efficient underwater robot capable of dynamic payloads. International journal of robotic research, 29 (2010), 547-570.

[Wat01] G. WATt. A quasy-steady evaluation of submarine rising stability: the stability limit. In RTO-AVT symposium on advanced flow management. Loen, Norway, 2001. 
[Wat07] G. WATt. Modelling and simulating unsteady six degrees-of-freedom submarine rising maneuvers. DRDC Atlantic TR 2007-08, 2007.

[YH10] Y. YANG and Y. HaO. Adaptive fuzzy modeling of hovering submarine based on on-line clustering. In 7th International Conference on Fuzzy Systems and Knowledge Discovery (FSKD 2010). Yantai, China, 2010.

[YJ10] X. YING and X. JIAN. Simulation of submarine hovering based on PID control. In 2nd International Asia Conference on Informatics in Control, Automation and Robotics. Wuhan, China, 2010. 